Supporting Information

for

\title{
Dephosphinylative [4 + 2] Benzannulation of Phosphinyl Ynamines: Application to the Modular Synthesis of Polycyclic Aromatic Amines
}

\author{
Yasuhiro Okuda, ${ }^{1}$ Mayo Fujimoto, ${ }^{1}$ Haruo Akashi, ${ }^{2}$ and Akihiro Orita ${ }^{1 *}$ \\ ${ }^{1}$ Department of Applied Chemistry and Biotechnology, Faculty of Engineering, ${ }^{2}$ Institute of \\ Frontier Science and Technology, Okayama University of Science, 1-1 Ridai-cho, Kita-ku, \\ Okayama 700-0005, Japan
}

Phone: $+81-86-256-9533$

Fax: +81-86-256-4292

E-mail: orita@dac.ous.ac.jp 


\section{Table of Contents}

1. Optimization Study (Table S1) S3

2. Attempted Pd-catalyzed coupling reactions of 4 a (Scheme S1, Table S2) S4-S5

3. Crystallographic Data (Figure S1 and Table S3) S6-S7

4. Electrochemical Properties (Figures S2-4, Table S4, Equation S1) S8-S11

$\begin{array}{ll}\text { 5. Theoretical Information (Figures S5-7) S12-S21 } & \text { S1 }\end{array}$

6. References and Notes $\quad S 22$

7. Copies of ${ }^{1} \mathrm{H},{ }^{13} \mathrm{C}\left\{{ }^{1} \mathrm{H}\right\},{ }^{19} \mathrm{~F}\left\{{ }^{1} \mathrm{H}\right\}$, and ${ }^{31} \mathrm{P}\left\{{ }^{1} \mathrm{H}\right\}$ NMR Charts (Figures S8-121) S23-S136 


\section{Optimization Study}

Table 1. Investigated reaction conditions on dephosphinylative [4 +2$]$ benzannulation of phosphinyl ynamine 1a with 2-bromobiphenyl 2a.

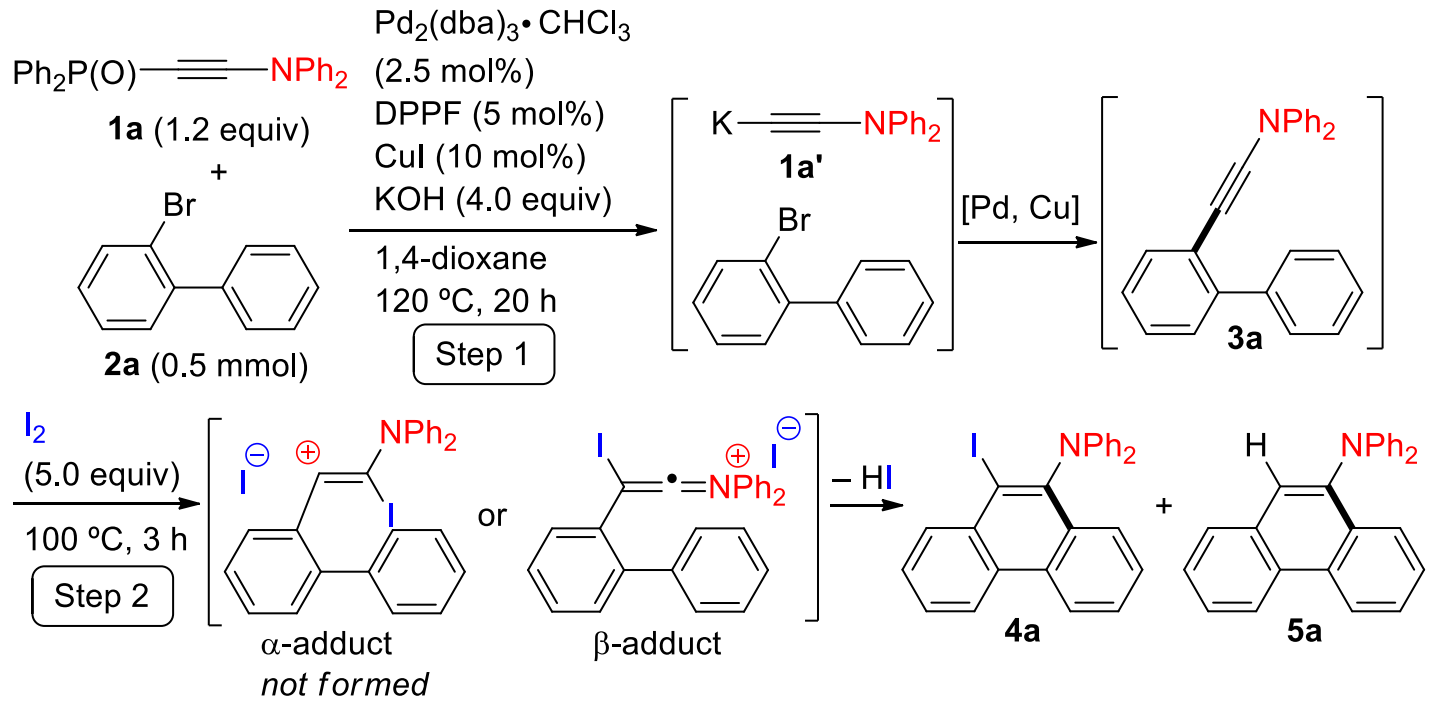

\begin{tabular}{|c|c|c|c|}
\hline \multirow{2}{*}{ entry } & \multirow{2}{*}{ deviations from the initial condition } & \multicolumn{2}{|c|}{ NMR yield (\%) } \\
\hline & & $4 a$ & $5 a$ \\
\hline 1 & none & $75(72)^{a}$ & 0 \\
\hline 2 & $\mathrm{Pd}_{2}(\mathrm{dba})_{3} \cdot \mathrm{CHCl}_{3}(1.25 \mathrm{~mol} \%)$, DPPF $(2.5 \mathrm{~mol} \%)$, Cul $(5 \mathrm{~mol} \%)$ & 65 & 0 \\
\hline 3 & $\mathrm{Pd}_{2}(\mathrm{dba})_{3} \cdot \mathrm{CHCl}_{3}(5 \mathrm{~mol} \%)$, DPPF $(10 \mathrm{~mol} \%)$, Cul $(20 \mathrm{~mol} \%)$ & 73 & 0 \\
\hline 4 & $\mathrm{Pd}(\mathrm{OAc})_{2}(5 \mathrm{~mol} \%)$ was used instead of $\mathrm{Pd}_{2}(\mathrm{dba})_{3} \cdot \mathrm{CHCl}_{3}$ & 44 & 0 \\
\hline 5 & $\mathrm{PdCl}_{2}(\mathrm{dppf})(5 \mathrm{~mol} \%)$ was used instead of $\mathrm{Pd}_{2}(\mathrm{dba})_{3} \cdot \mathrm{CHCl}_{3} / \mathrm{DPPF}$ & 18 & 0 \\
\hline 6 & $\mathrm{PPh}_{3}(10 \mathrm{~mol} \%)$ instead of DPPF & 11 & 0 \\
\hline 7 & DPPE $(5 \mathrm{~mol} \%)$ instead of DPPF & 12 & 1 \\
\hline 8 & xantphos (5 mol\%) instead of DPPF & 72 & 0 \\
\hline 9 & bpy $(5 \mathrm{~mol} \%)$ instead of DPPF & trace & trace \\
\hline 10 & $\mathrm{MeOH}$ was used instead of 1,4-dioxane & 0 & 0 \\
\hline 11 & $\mathrm{H}_{2} \mathrm{O}$ was used instead of 1,4-dioxane & 0 & 0 \\
\hline 12 & without $\mathrm{Pd}_{2}(\mathrm{dba})_{3} \cdot \mathrm{CHCl}_{3}$ & trace & 0 \\
\hline 13 & without DPPF & 11 & 0 \\
\hline 14 & without Cul & 38 & 4 \\
\hline
\end{tabular}

${ }^{a}$ An isolated yield. 


\section{Attempted Pd-catalyzed coupling reactions of 4a}

We also investigated other palladium-catalyzed cross-coupling of 4a; (a) SonogashiraHagihara coupling with phenylacetylene, (b) Buchwald-Hartwig coupling with morpholine, (c) and B-alkyl Suzuki-Miyaura coupling with $B$ - $n$-decyl-(9-BBN). However, deiodized (9amino)phenanthrene $\mathbf{5 \mathbf { a } ^ { 1 }}$ was exclusively detected in $51 \%, 68 \%$, and $88 \%$ yields, respectively.

(a)

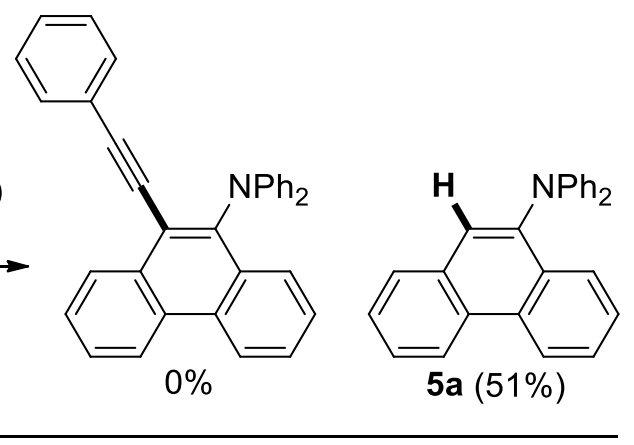

(b)

$(0.5 \mathrm{mmol})$

toluene

$110^{\circ} \mathrm{C}, 24 \mathrm{~h}$

$\mathrm{Pd}\left(\mathrm{PPh}_{3}\right)_{4}(5 \mathrm{~mol} \%)$

Cul (10 mol\%)

${ }^{i} \mathrm{Pr}_{2} \mathrm{EtN}$ (3.0 equiv)

$\mathrm{Pd}_{2}(\mathrm{dba})_{3} \cdot \mathrm{CHCl}_{3}$

$(1 \mathrm{~mol} \%)$

xantphos (4 mol \%)

$\mathrm{NaO}{ }^{t} \mathrm{Bu}$ ( 1.5 equiv)

1,4-dioxane

$80^{\circ} \mathrm{C}, 24 \mathrm{~h}$

$4 a$

(0.5 mmol)

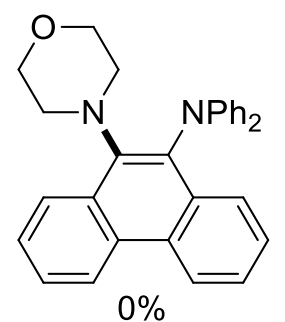

$0 \%$

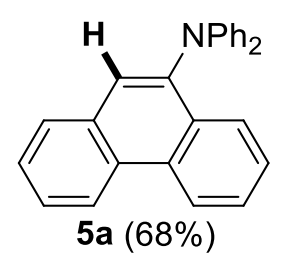

(c)
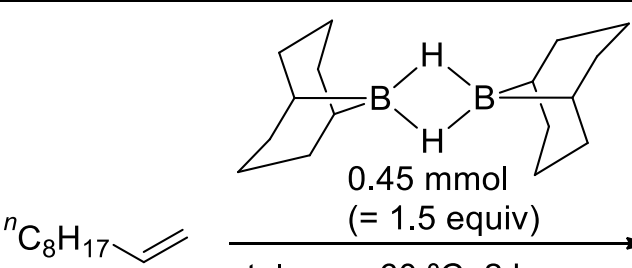

$0.9 \mathrm{mmol}$ (= 3.0 equiv)
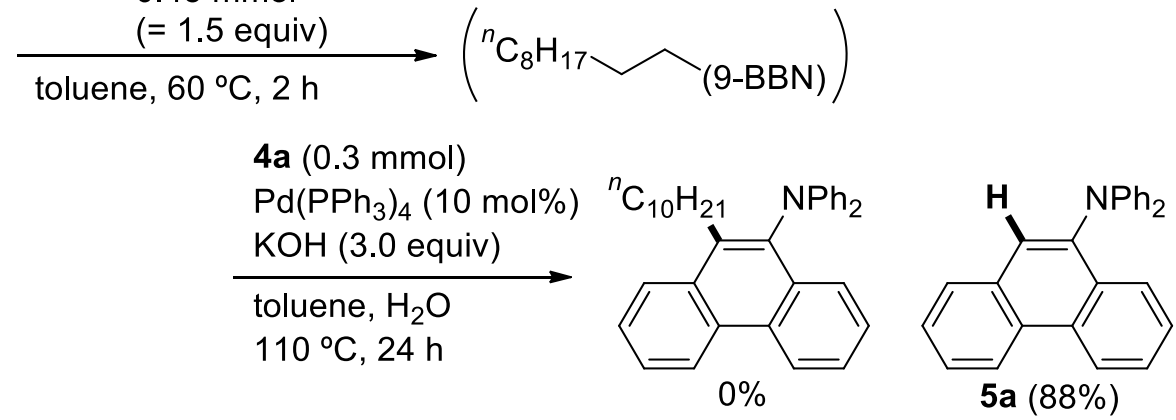

Scheme S1. Failed examples in palladium-catalyzed cross-coupling of $\mathbf{4 a}$. 
In addition, the amount of boronic acid was also investigated.

Table S2. Effect of the amount of phenyl boronic acid.

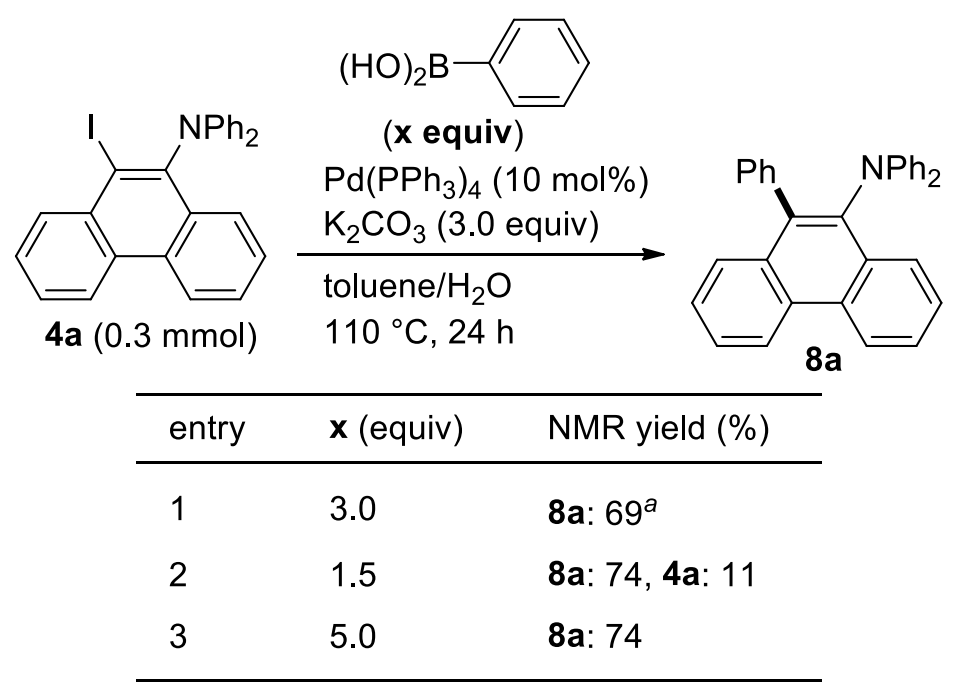

${ }^{a}$ An isolated yield. 


\section{Crystallographic Data}

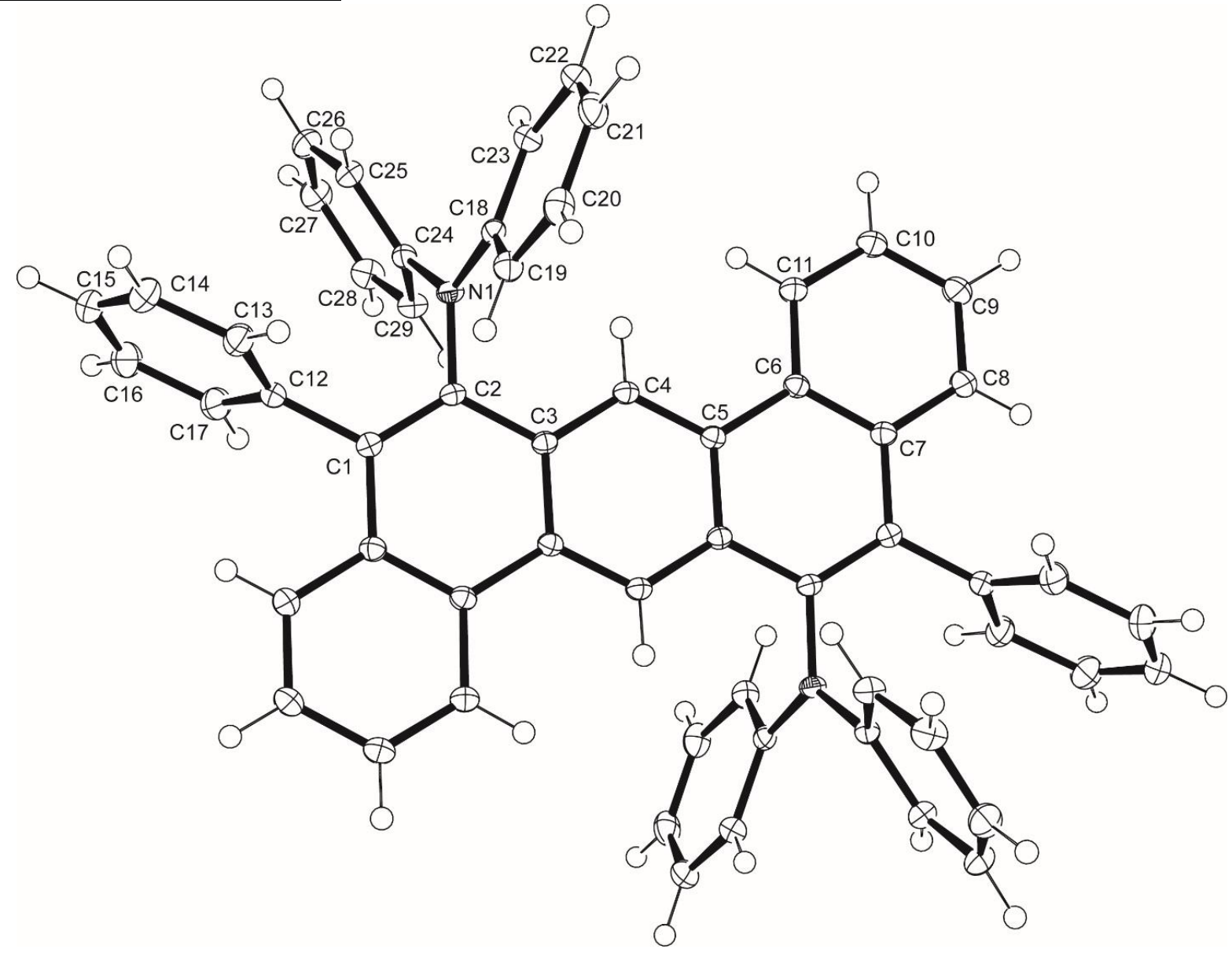

Figure S1. ORTEP drawing of 13a at 50\% ellipsoid probability (CCDC 2093035).

Single crystals of 13a were obtained by slow evaporation of solution of $5 \mathrm{mg}$ of 13a in $1 \mathrm{~mL}$ of chloroform. 
Table S3. Crystal data and structure refinement for 13a.

\begin{tabular}{|c|c|}
\hline Empirical formula & $\mathrm{C}_{30} \mathrm{H}_{21} \mathrm{Cl}_{3} \mathrm{~N}$ \\
\hline Formula weight & 501.83 \\
\hline Temperature/K & 293(2) \\
\hline Crystal system & monoclinic \\
\hline Space group & $\mathrm{P} 2{ }_{1} / \mathrm{c}$ \\
\hline $\mathrm{a} / \AA$ & $10.5541(2)$ \\
\hline $\mathrm{b} / \AA$ & $23.8728(4)$ \\
\hline $\mathrm{c} / \AA$ & $9.64810(10)$ \\
\hline$\alpha /^{\circ}$ & 90 \\
\hline$\beta /{ }^{\circ}$ & $91.7700(10)$ \\
\hline$\gamma /{ }^{\circ}$ & 90 \\
\hline Volume/ $\AA^{3}$ & $2429.74(7)$ \\
\hline $\mathrm{Z}$ & 4 \\
\hline$\rho_{\text {calc }} \mathrm{g} / \mathrm{cm}^{3}$ & 1.372 \\
\hline$\mu / \mathrm{mm}^{-1}$ & 0.397 \\
\hline $\mathrm{F}(000)$ & 1036.0 \\
\hline Crystal size $/ \mathrm{mm}^{3}$ & $0.2 \times 0.15 \times 0.08$ \\
\hline Radiation & $\operatorname{MoK} \alpha(\lambda=0.71073)$ \\
\hline $2 \Theta$ range for data collection $/^{\circ}$ & 4.222 to 63.054 \\
\hline Index ranges & $-14 \leq \mathrm{h} \leq 14,-34 \leq \mathrm{k} \leq 34,-13 \leq 1 \leq 13$ \\
\hline Reflections collected & 44945 \\
\hline Independent reflections & $7590\left[\mathrm{R}_{\text {int }}=0.0332, \mathrm{R}_{\text {sigma }}=0.0169\right]$ \\
\hline Data/restraints/parameters & $7590 / 0 / 307$ \\
\hline Goodness-of-fit on $\mathrm{F}^{2}$ & 1.044 \\
\hline Final $\mathrm{R}$ indexes $[\mathrm{I}>=2 \sigma(\mathrm{I})]$ & $\mathrm{R}_{1}=0.0364, \mathrm{wR}_{2}=0.0978$ \\
\hline Final $\mathrm{R}$ indexes [all data] & $\mathrm{R}_{1}=0.0403, \mathrm{w} \mathrm{R}_{2}=0.1004$ \\
\hline Largest diff. peak/hole / e $\AA^{-3}$ & $0.52 /-0.39$ \\
\hline
\end{tabular}




\section{Electrochemical Properties}

Cyclic voltammograms were obtained under the following conditions; solvent: dichloromethane (concentration: $0.5 \mathrm{mM}$ ), electrolyte: $0.1 \mathrm{M}^{n} \mathrm{Bu}_{4} \mathrm{NPF}_{6}$, working electrode: glassy carbon, counter electrode: Pt, reference electrode: $\mathrm{Ag} / \mathrm{AgNO}_{3}$, scan rate: $100 \mathrm{mV} / \mathrm{s}$.
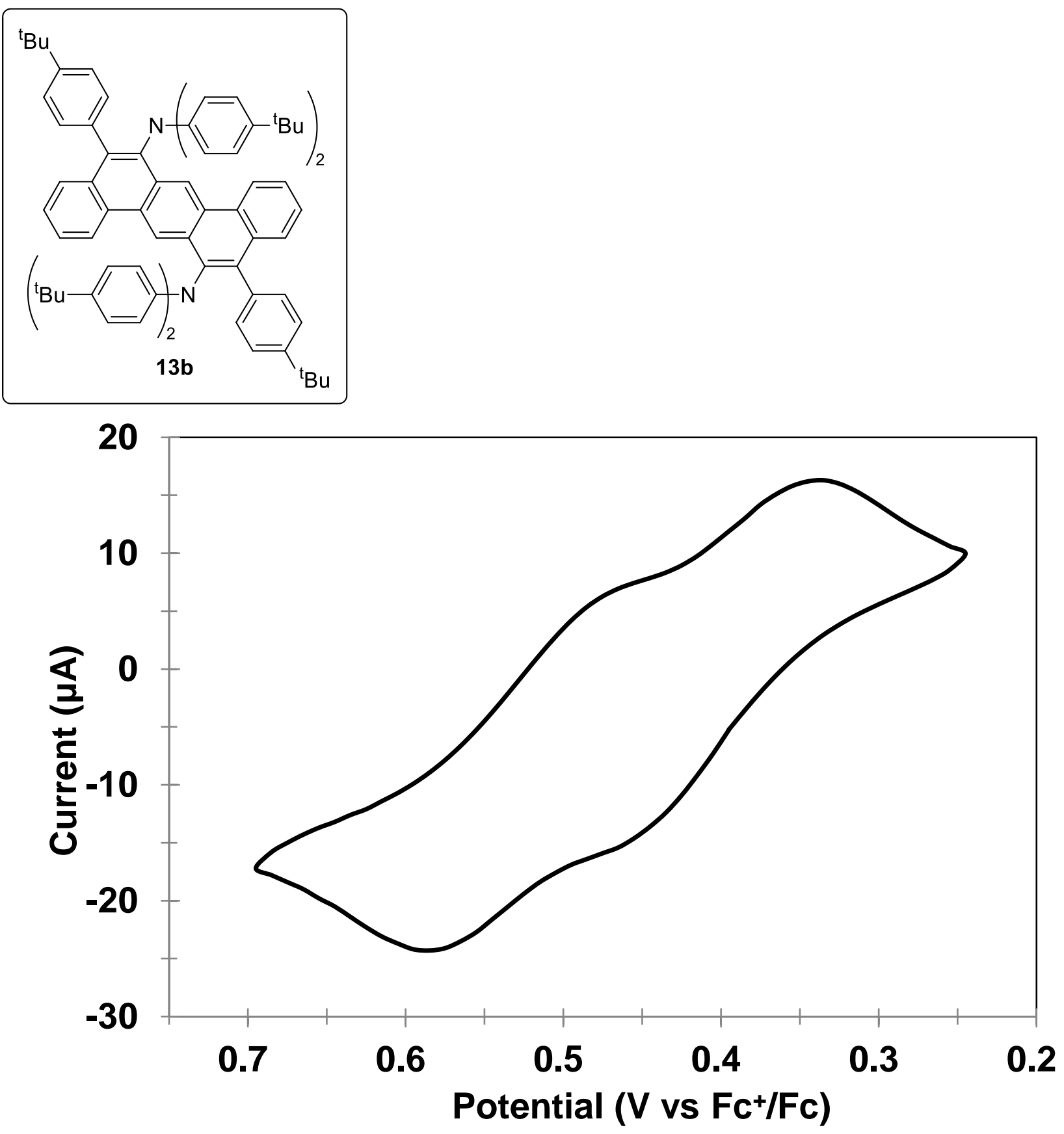

Figure S2. Cyclic voltammogram of $\mathbf{1 3 b}$. 

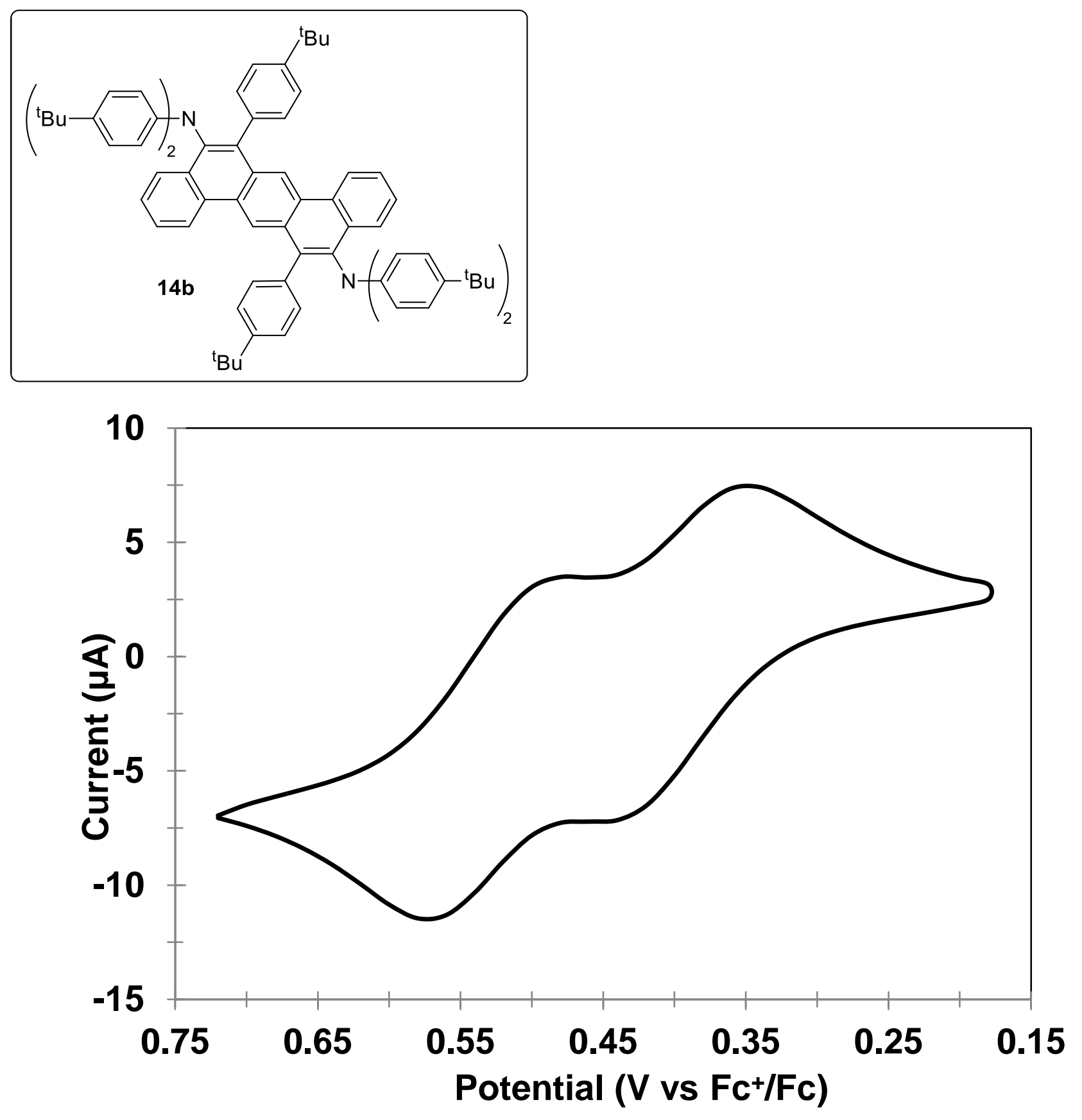

Figure S3. Cyclic voltammogram of $\mathbf{1 4 b}$. 

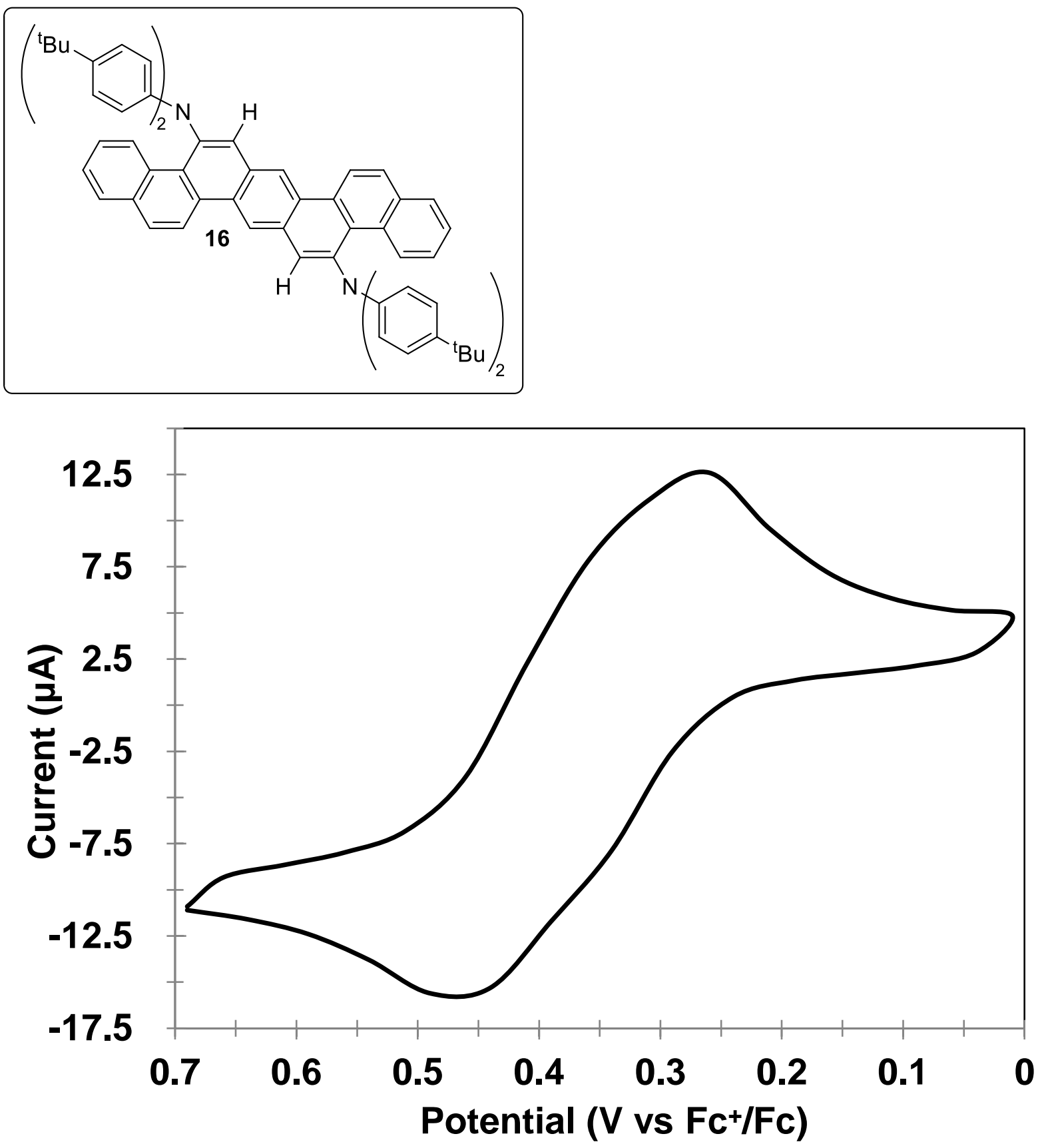

Figure S4. Cyclic voltammogram of $\mathbf{1 6 .}$ 
Table S4. Oxidation/Reduction Potentials of Compounds 13b, 14b, and 16.

\begin{tabular}{llccc}
\hline entry & compound & $E_{1 / 2}{ }^{\text {ox }}\left(\mathrm{vs} \mathrm{Fc} / \mathrm{Fc}^{+}\right) / \mathrm{V}$ & $E_{1 / 2}^{\text {red }}\left(\mathrm{vs} \mathrm{Fc} / \mathrm{Fc}^{+}\right) / \mathrm{V}$ & $\mathrm{HOMO} / \mathrm{eV}$ \\
\hline $\mathrm{I}$ & $\mathrm{I} \mathbf{3 a}$ & $+0.40,+0.53$ & $\mathrm{ND}$ & -5.20 \\
3 & $\mathrm{I4a}$ & $+0.39,+0.53$ & $\mathrm{ND}$ & -5.19 \\
4 & 16 & $+0.35,+0.40$ & $\mathrm{ND}$ & -5.15 \\
\hline
\end{tabular}

The HOMO levels of $\mathbf{1 3 b}, \mathbf{1 4 b}$, and 16 were experimentally estimated from their oxidation potentials, respectively, by taking advantage of Equation S1.

$$
E_{\mathrm{HOMO}}=-4.8-E_{1 / 2}{ }^{\mathrm{ox}}(\text { Equation S1) }
$$




\section{Theoretical Information}

\section{Computational Methodology}

All calculations in the present study were performed with the Gaussian 09 program $^{2}$ and by using the Becke-three-parameter plus Lee-Yang-Parr (B3LYP) density functional theory (DFT) method. ${ }^{3,4}$ For all geometry optimizations and normal coordinate analyses at stationary points, we used $6-31 \mathrm{G}(\mathrm{d})$ basis set $^{5,6}$ for all atoms. The dispersion energy was corrected by using gaussian command $\operatorname{IOp}(3 / 124=3){ }^{7}$

\section{Calculated HOMO and LUMO of 13a, 14a, and 16'}
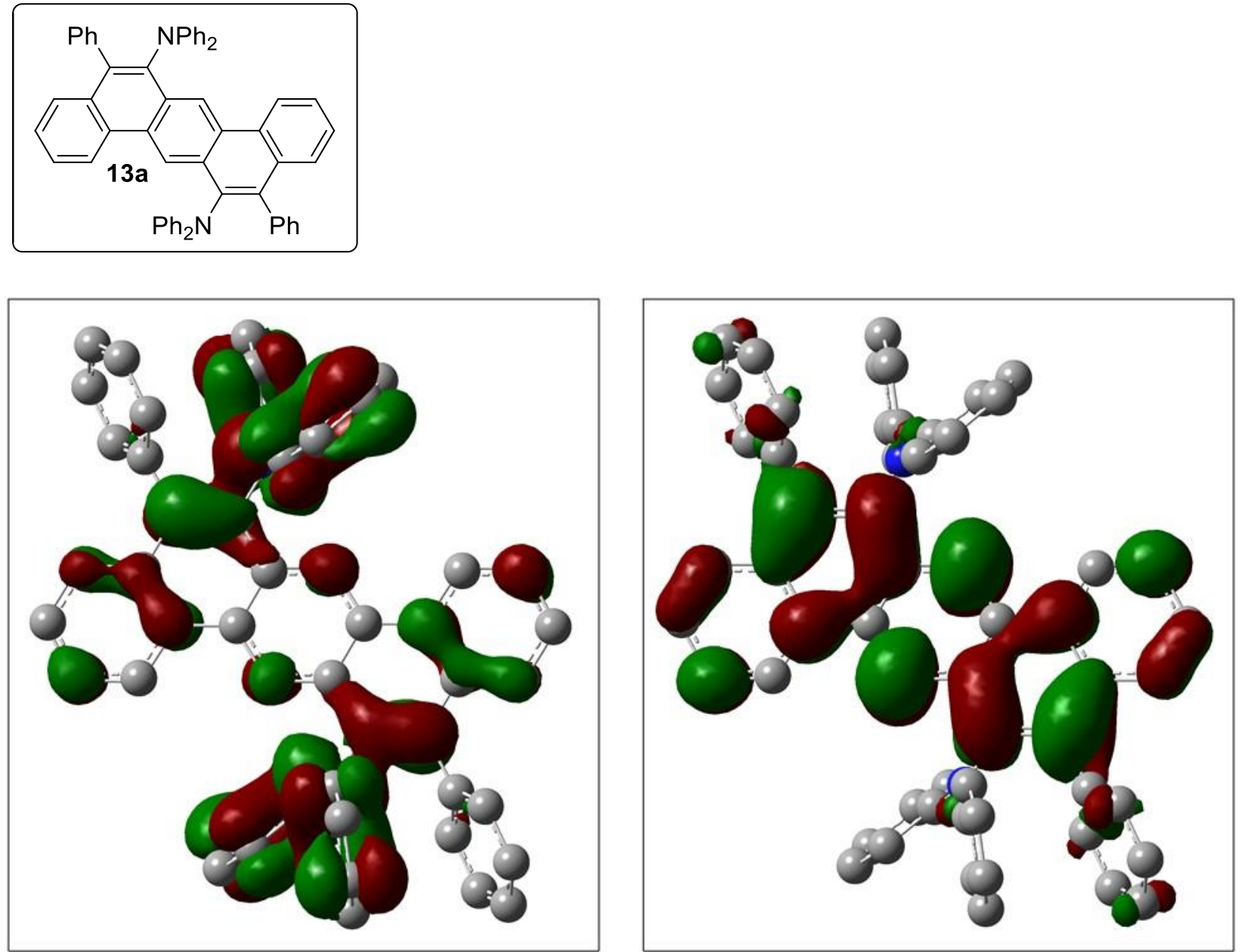

Figure S5. Calculated HOMO (left) and LUMO (right) of 13a. Hydrogen atoms were omitted for clarity. 

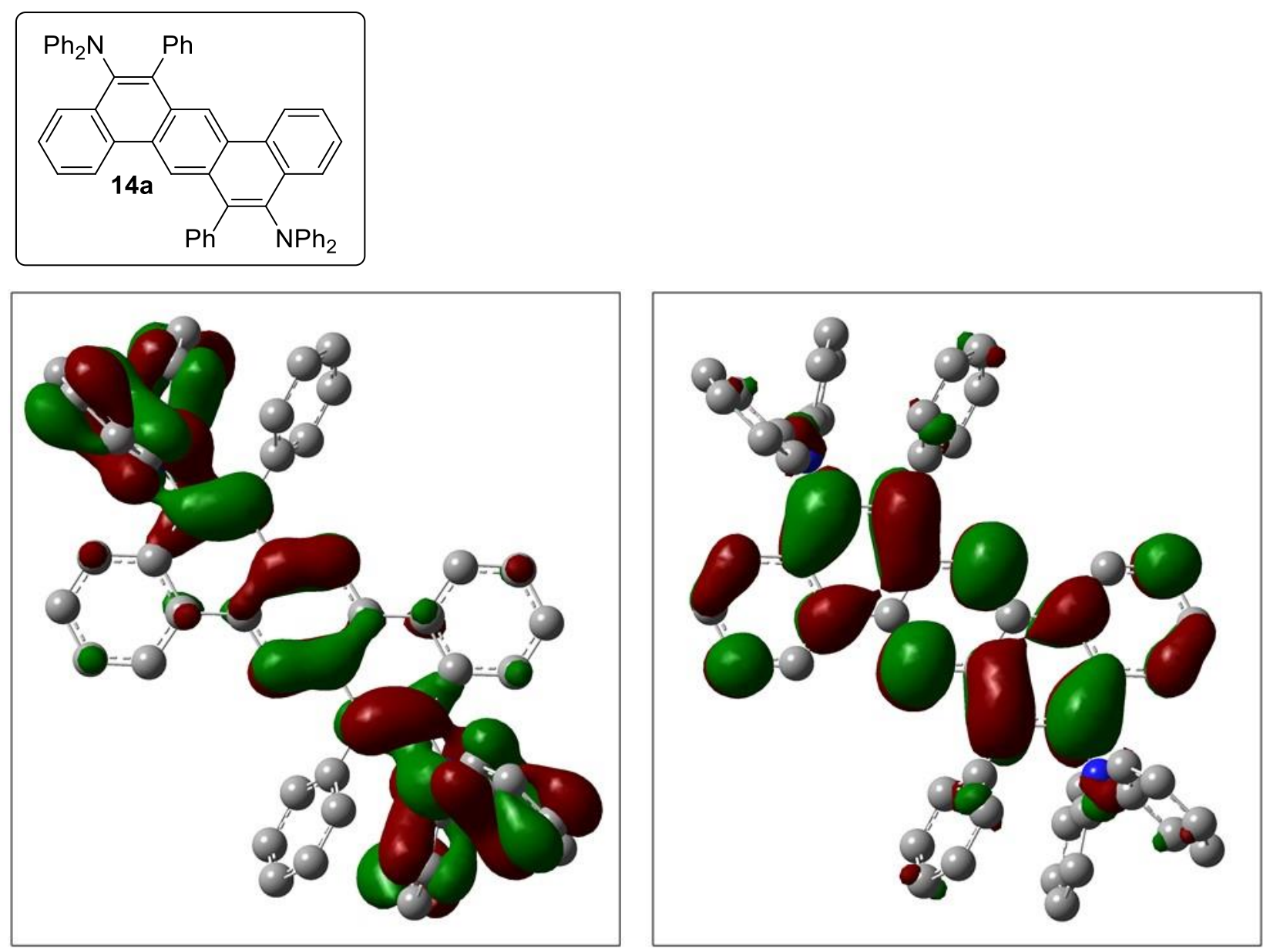

Figure S6. Calculated HOMO (left) and LUMO (right) of 14a. Hydrogen atoms were omitted for clarity. 

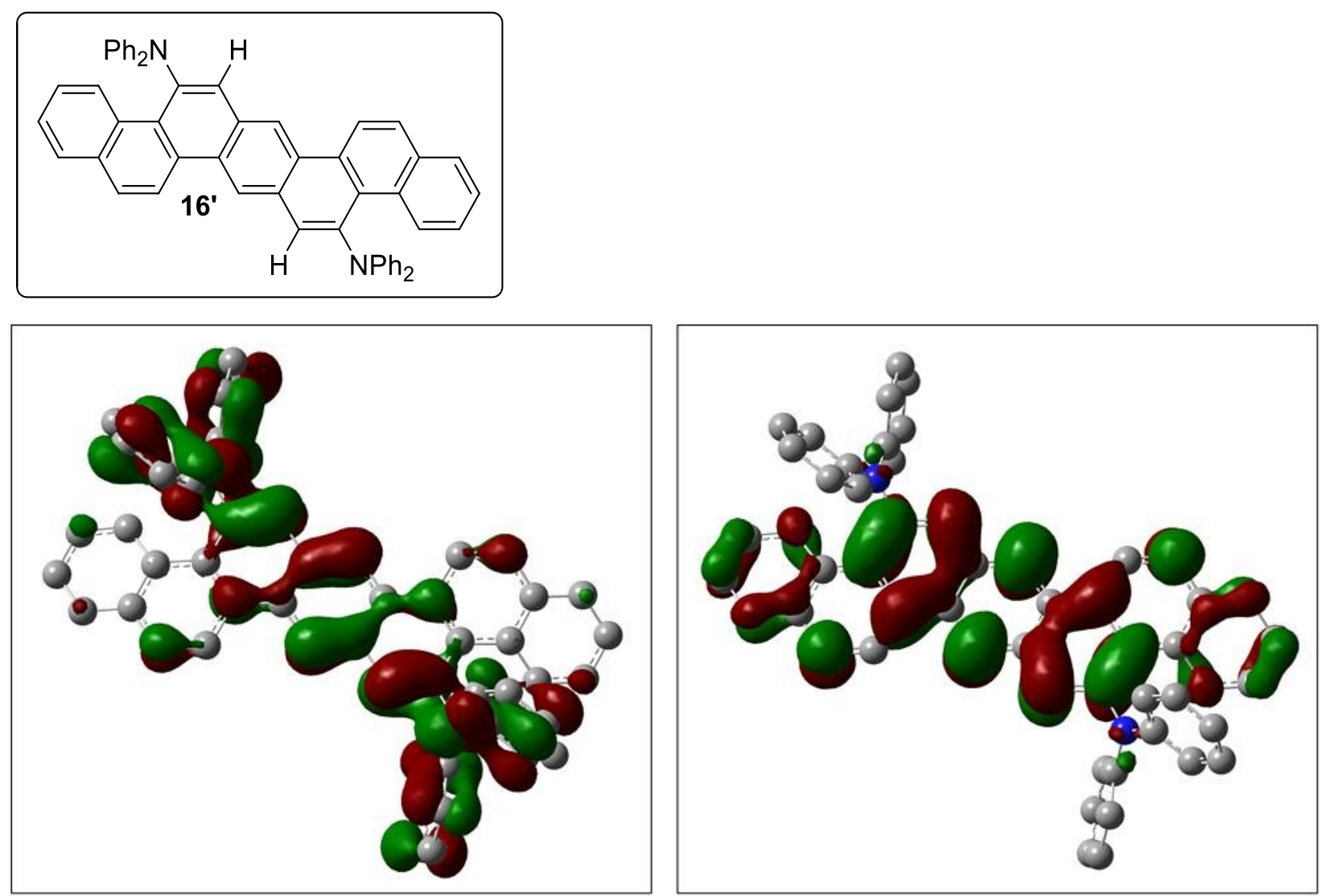

Figure S7. Calculated HOMO (left) and LUMO (right) of 16' ( ${ }^{t} \mathrm{Bu}$ groups of $\mathbf{1 6}$ were replaced by hydrogen atoms). Hydrogen atoms were omitted for clarity.

\section{Cartesian Coordinate for Optimized Structure of 13a}

SCF Done: E(RB3LYP) $=-2343.94547179$ (hartree)

Zero-point correction $=0.806111$ (hartree)

Thermal correction to Energy $=0.851823$ (hartree)

Thermal correction to Enthalpy $=0.852767$ (hartree)

Thermal correction to Gibbs Free Energy $=0.723865$ (hartree)

The number of imaginary frequencies: 0

$\begin{array}{lrrr}C & -4.88335100 & -1.70449600 & 0.29674500 \\ C & -3.73943500 & -0.94194300 & 0.12844600 \\ C & -2.46777100 & -1.54254100 & -0.00891900 \\ C & -1.25388100 & -0.75906600 & -0.21008300 \\ C & -1.26326900 & 0.63446400 & -0.31243900 \\ C & -0.09143900 & 1.38793400 & -0.45308300\end{array}$




\begin{tabular}{|c|c|c|c|}
\hline $\mathrm{C}$ & -0.13605700 & 2.83150400 & -0.53279600 \\
\hline $\mathrm{C}$ & 1.17227600 & 0.72088600 & -0.52752400 \\
\hline $\mathrm{C}$ & 1.00400600 & 3.58082900 & -0.68194400 \\
\hline $\mathrm{C}$ & 0.00983300 & -1.42611400 & -0.28452500 \\
\hline $\mathrm{C}$ & 1.18166300 & -0.67264300 & -0.42516800 \\
\hline $\mathrm{C}$ & 2.38616600 & 1.50436200 & -0.72868800 \\
\hline $\mathrm{C}$ & 2.29477500 & 2.92721400 & -0.79730000 \\
\hline $\mathrm{C}$ & 3.65783000 & 0.90376300 & -0.86605200 \\
\hline $\mathrm{C}$ & 3.48071700 & 3.68430400 & -0.95112400 \\
\hline $\mathrm{C}$ & 4.80174600 & 1.66631600 & -1.03435000 \\
\hline $\mathrm{C}$ & 4.71456700 & 3.06856600 & -1.06706200 \\
\hline $\mathrm{H}$ & 3.41154900 & 4.76582300 & -0.97673500 \\
\hline $\mathrm{H}$ & 5.76548900 & 1.17537100 & -1.13893000 \\
\hline $\mathrm{H}$ & 5.61169400 & 3.67015200 & -1.18609100 \\
\hline $\mathrm{C}$ & -2.37638000 & -2.96539400 & 0.05969200 \\
\hline $\mathrm{C}$ & -1.08561200 & -3.61900800 & -0.05566600 \\
\hline $\mathrm{C}$ & -3.56232100 & -3.72248400 & 0.21351600 \\
\hline $\mathrm{C}$ & 0.05445100 & -2.86968300 & -0.20481300 \\
\hline $\mathrm{C}$ & -4.79617200 & -3.10674600 & 0.32945600 \\
\hline $\mathrm{H}$ & 3.75179700 & -0.17643800 & -0.85438900 \\
\hline $\mathrm{H}$ & 2.11938000 & -1.20700800 & -0.45713900 \\
\hline $\mathrm{H}$ & -3.83340300 & 0.13825800 & 0.11678500 \\
\hline $\mathrm{H}$ & -5.84709400 & -1.21355100 & 0.40132700 \\
\hline $\mathrm{H}$ & -5.69329900 & -3.70833300 & 0.44848600 \\
\hline $\mathrm{H}$ & -3.49315400 & -4.80400300 & 0.23912700 \\
\hline $\mathrm{H}$ & -2.20098600 & 1.16882800 & -0.28046800 \\
\hline $\mathrm{C}$ & 0.89115500 & 5.06331900 & -0.75315200 \\
\hline $\mathrm{C}$ & 1.18760900 & 5.74247500 & -1.94301800 \\
\hline $\mathrm{C}$ & 0.41514600 & 5.79075300 & 0.34595000 \\
\hline $\mathrm{C}$ & 1.00814600 & 7.12329600 & -2.03452200 \\
\hline $\mathrm{H}$ & 1.53545400 & 5.17644200 & -2.80208800 \\
\hline $\mathrm{C}$ & 0.23920900 & 7.17215900 & 0.25661100 \\
\hline $\mathrm{H}$ & 0.16129100 & 5.26465600 & 1.26119300 \\
\hline $\mathrm{C}$ & 0.53415300 & 7.84204200 & -0.93418300 \\
\hline $\mathrm{H}$ & 1.22910400 & 7.63523400 & -2.96731200 \\
\hline $\mathrm{H}$ & -0.13215300 & 7.72455900 & 1.11604900 \\
\hline $\mathrm{H}$ & 0.39184300 & 8.91728200 & -1.00511300 \\
\hline $\mathrm{C}$ & -0.97276100 & -5.10149800 & 0.01554100 \\
\hline $\mathrm{C}$ & -0.49675600 & -5.82893200 & -1.08356300 \\
\hline $\mathrm{C}$ & -1.26921300 & -5.78065500 & 1.20540800 \\
\hline $\mathrm{C}$ & -0.32082000 & -7.21033900 & -0.99422500 \\
\hline $\mathrm{H}$ & -0.24290400 & -5.30283500 & -1.99880600 \\
\hline $\mathrm{C}$ & -1.08975100 & -7.16147600 & 1.29691000 \\
\hline $\mathrm{H}$ & -1.61705500 & -5.21462300 & 2.06447800 \\
\hline $\mathrm{C}$ & -0.61576200 & -7.88022200 & 0.19656900 \\
\hline
\end{tabular}




$\begin{array}{lrrr}\mathrm{H} & 0.05053900 & -7.76273800 & -1.85366500 \\ \mathrm{H} & -1.31070700 & -7.67341400 & 2.22970000 \\ \mathrm{H} & -0.47345300 & -8.95546200 & 0.26749800 \\ \mathrm{~N} & 1.33018000 & -3.51387600 & -0.23293400 \\ \mathrm{~N} & -1.41178500 & 3.47569700 & -0.50467600 \\ \mathrm{C} & -2.17138200 & 3.46865300 & 0.67876700 \\ \mathrm{C} & -3.56794600 & 3.64571800 & 0.64408700 \\ \mathrm{C} & -1.55224200 & 3.22152800 & 1.91737200 \\ \mathrm{C} & -4.31239300 & 3.59493500 & 1.82099700 \\ \mathrm{H} & -4.05821600 & 3.82331800 & -0.30686900 \\ \mathrm{C} & -2.31112500 & 3.16021100 & 3.08574300 \\ \mathrm{H} & -0.47942300 & 3.06509700 & 1.95426600 \\ \mathrm{C} & -3.69426100 & 3.35100200 & 3.05163700 \\ \mathrm{H} & -5.38914500 & 3.73679800 & 1.77076900 \\ \mathrm{H} & -1.81050700 & 2.96478300 & 4.03065000 \\ \mathrm{H} & -4.28081800 & 3.30654000 & 3.96466200 \\ \mathrm{C} & -1.80179400 & 4.19943800 & -1.66112600 \\ \mathrm{C} & -2.36932500 & 5.47743800 & -1.56103600 \\ \mathrm{C} & -1.55385300 & 3.65101600 & -2.92738500 \\ \mathrm{C} & -2.68494700 & 6.19168100 & -2.71483400 \\ \mathrm{H} & -2.53025500 & 5.90918700 & -0.58000200 \\ \mathrm{C} & -1.85909500 & 4.37900600 & -4.07704100 \\ \mathrm{H} & -1.11262000 & 2.66143000 & -2.99715200 \\ \mathrm{C} & -2.42868400 & 5.65143200 & -3.97888000 \\ \mathrm{H} & -3.11274700 & 7.18672100 & -2.62374200 \\ \mathrm{H} & -1.65857800 & 3.94417700 & -5.05292500 \\ \mathrm{H} & -2.66801200 & 6.21628400 & -4.87561700 \\ \mathrm{C} & 2.08977700 & -3.50682800 & -1.41637700 \\ \mathrm{C} & 3.48634100 & -3.68389100 & -1.38169600 \\ \mathrm{C} & 1.47063700 & -3.25970200 & -2.65498100 \\ \mathrm{C} & 4.23078900 & -3.63310400 & -2.55860600 \\ \mathrm{H} & 3.97661100 & -3.86149200 & -0.43074000 \\ \mathrm{C} & 2.22952000 & -3.19838100 & -3.82335200 \\ \mathrm{H} & 0.39781900 & -3.10327200 & -2.69187500 \\ \mathrm{C} & 3.61265700 & -3.38917000 & -3.78924500 \\ \mathrm{H} & 5.30754100 & -3.77496600 & -2.50837700 \\ \mathrm{H} & 1.72890300 & -3.00295200 & -4.76825900 \\ \mathrm{C} & 4.19921400 & -3.34470500 & -4.70227000 \\ \mathrm{H} & 1.72018900 & -4.23761700 & 0.92351500 \\ \mathrm{H} & 2.4772300 & -5.51561600 & 0.82342300 \\ \mathrm{H} & -3.68919800 & 2.18977500 \\ \mathrm{H} & -634700 & -6.22986000 & 1.97722000 \\ \mathrm{H} & -5.94736300 & -0.15761100 \\ \mathrm{H} & -4.41718900 & 3.33943000 \\ \mathrm{H} & -2.69961300 & 2.25954300\end{array}$




$\begin{array}{llll}\mathrm{C} & 2.34708200 & -5.68961400 & 3.24126700 \\ \mathrm{H} & 3.03114900 & -7.22489900 & 1.88612700 \\ \mathrm{H} & 1.57697300 & -3.98236200 & 4.31531400 \\ \mathrm{H} & 2.58641200 & -6.25446600 & 4.13800300\end{array}$

\section{Cartesian Coordinate for Optimized Structure of 14a}

SCF Done: E $($ RB3LYP $)=-2343.94409265$ (hartree)

Zero-point correction $=0.806225$ (hartree)

Thermal correction to Energy $=0.851882$ (hartree)

Thermal correction to Enthalpy $=0.852826$ (hartree)

Thermal correction to Gibbs Free Energy $=0.724230$ (hartree)

The number of imaginary frequencies: 0

$\begin{array}{lrrr}\mathrm{C} & -5.08066000 & -1.18763500 & -0.43765300 \\ \mathrm{C} & -3.85325100 & -0.54620000 & -0.44200500 \\ \mathrm{C} & -2.64176500 & -1.27424400 & -0.45072600 \\ \mathrm{C} & -1.33415900 & -0.62694700 & -0.42560300 \\ \mathrm{C} & -1.18355400 & 0.76224800 & -0.42679800 \\ \mathrm{C} & 0.06782100 & 1.39450100 & -0.39494600 \\ \mathrm{C} & 0.17248100 & 2.83921700 & -0.41428300 \\ \mathrm{C} & 1.25346800 & 0.58885300 & -0.39190300 \\ \mathrm{C} & 1.40878800 & 3.43436300 & -0.40838200 \\ \mathrm{C} & -0.14875800 & -1.43264600 & -0.40246500 \\ \mathrm{C} & 1.10299800 & -0.80032500 & -0.40157700 \\ \mathrm{C} & 2.56125800 & 1.23621700 & -0.38293100 \\ \mathrm{C} & 2.63213200 & 2.65911500 & -0.38520100 \\ \mathrm{C} & 3.77220900 & 0.50812200 & -0.34702500 \\ \mathrm{C} & 3.89378500 & 3.29490900 & -0.33158700 \\ \mathrm{C} & 4.99911300 & 1.14950900 & -0.31067800 \\ \mathrm{C} & 5.06153900 & 2.55396200 & -0.29810300 \\ \mathrm{H} & 3.93537200 & 4.37746400 & -0.31714600 \\ \mathrm{H} & 5.91297100 & 0.56214200 & -0.28467300 \\ \mathrm{H} & 6.02300100 & 3.05861600 & -0.25944800 \\ \mathrm{C} & -2.71252600 & -2.69711400 & -0.45944400 \\ \mathrm{C} & -1.48897100 & -3.47235300 & -0.45562900 \\ \mathrm{C} & -3.97509400 & -3.33301200 & -0.43854800 \\ \mathrm{C} & -0.25287800 & -2.87729700 & -0.42942200 \\ \mathrm{C} & -5.14333800 & -2.59212000 & -0.43109800 \\ \mathrm{C} & -1.05024500 & 3.68786300 & -0.42602200 \\ \mathrm{C} & -1.90613100 & 3.71307100 & 0.68409200 \\ \mathrm{C} & -1.32944100 & 4.51632900 & -1.52091100 \\ \mathrm{C} & -3.01852900 & 4.55531500 & 0.70229200 \\ \mathrm{H} & -1.67899400 & 3.08538700 & 1.54077600\end{array}$




\begin{tabular}{|c|c|c|c|}
\hline $\mathrm{C}$ & -2.44356900 & 5.35670800 & -1.50467700 \\
\hline $\mathrm{H}$ & -0.65686700 & 4.51525100 & -2.37310100 \\
\hline $\mathrm{C}$ & -3.29029100 & 5.37941900 & -0.39294100 \\
\hline $\mathrm{H}$ & -3.66601200 & 4.57503000 & 1.57488500 \\
\hline $\mathrm{H}$ & -2.64807600 & 5.99618900 & -2.35958400 \\
\hline $\mathrm{C}$ & 0.96979600 & -3.72596600 & -0.41459500 \\
\hline $\mathrm{C}$ & 1.27511000 & -4.55093000 & -1.50514900 \\
\hline $\mathrm{C}$ & 1.79891500 & -3.75477200 & 0.71556400 \\
\hline $\mathrm{C}$ & 2.38859100 & -5.39135600 & -1.46500900 \\
\hline $\mathrm{H}$ & 0.62305800 & -4.54716600 & -2.37313600 \\
\hline $\mathrm{C}$ & 2.91063800 & -4.59705500 & 0.75762200 \\
\hline $\mathrm{H}$ & 1.55135300 & -3.12979400 & 1.56856000 \\
\hline $\mathrm{C}$ & 3.20855000 & -5.41762400 & -0.33345900 \\
\hline $\mathrm{H}$ & 2.61349600 & -6.02810400 & -2.31682800 \\
\hline $\mathrm{H}$ & 3.53709900 & -4.61956200 & 1.64536200 \\
\hline $\mathrm{N}$ & 1.50696800 & 4.86009600 & -0.36608900 \\
\hline $\mathrm{N}$ & -1.58821000 & -4.89823000 & -0.42102000 \\
\hline $\mathrm{C}$ & 0.96203300 & 5.51236000 & 0.77067900 \\
\hline $\mathrm{C}$ & 0.14383900 & 6.64371900 & 0.64209700 \\
\hline $\mathrm{C}$ & 1.19344900 & 4.96956000 & 2.04268500 \\
\hline $\mathrm{C}$ & -0.42976800 & 7.22098400 & 1.77292800 \\
\hline $\mathrm{H}$ & -0.05821800 & 7.04140500 & -0.34552300 \\
\hline $\mathrm{C}$ & 0.60328400 & 5.54484700 & 3.16764300 \\
\hline $\mathrm{H}$ & 1.82655400 & 4.09230700 & 2.13495800 \\
\hline $\mathrm{C}$ & -0.20893300 & 6.67505000 & 3.04117200 \\
\hline $\mathrm{H}$ & -1.07246800 & 8.08994200 & 1.65778100 \\
\hline $\mathrm{H}$ & 0.78787600 & 5.11178200 & 4.14746600 \\
\hline $\mathrm{C}$ & 1.97574800 & 5.55732400 & -1.49418600 \\
\hline $\mathrm{C}$ & 1.99598100 & 4.93493900 & -2.75590800 \\
\hline $\mathrm{C}$ & 2.47710800 & 6.86801500 & -1.37969400 \\
\hline $\mathrm{C}$ & 2.49574700 & 5.61120000 & -3.86870300 \\
\hline $\mathrm{H}$ & 1.62794300 & 3.91953200 & -2.85607300 \\
\hline $\mathrm{C}$ & 2.96052500 & 7.53675900 & -2.50205900 \\
\hline $\mathrm{H}$ & 2.48285100 & 7.35300000 & -0.40979000 \\
\hline $\mathrm{C}$ & 2.97566700 & 6.91772400 & -3.75615100 \\
\hline $\mathrm{H}$ & 2.50240100 & 5.10824500 & -4.83240300 \\
\hline $\mathrm{H}$ & 3.34022800 & 8.54927500 & -2.38971000 \\
\hline $\mathrm{C}$ & -1.07070300 & -5.55485100 & 0.72601800 \\
\hline $\mathrm{C}$ & -0.24990800 & -6.68598300 & 0.61284500 \\
\hline $\mathrm{C}$ & -1.33221400 & -5.01662400 & 1.99413200 \\
\hline $\mathrm{C}$ & 0.29648600 & -7.26752100 & 1.75490600 \\
\hline $\mathrm{H}$ & -0.02442900 & -7.08013300 & -0.37111400 \\
\hline $\mathrm{C}$ & -0.76921100 & -5.59623700 & 3.13073200 \\
\hline $\mathrm{H}$ & -1.96712000 & -4.13949800 & 2.07449900 \\
\hline $\mathrm{C}$ & 0.04556200 & -6.72620100 & 3.01952300 \\
\hline
\end{tabular}




$\begin{array}{lrrr}\mathrm{H} & 0.94159400 & -8.13622300 & 1.65192200 \\ \mathrm{H} & -0.97704900 & -5.16671300 & 4.10745300 \\ \mathrm{C} & -2.02943300 & -5.59111000 & -1.56284300 \\ \mathrm{C} & -2.01923500 & -4.96380500 & -2.82225400 \\ \mathrm{C} & -2.53309100 & -6.90232100 & -1.46569400 \\ \mathrm{C} & -2.49154800 & -5.63582900 & -3.94951000 \\ \mathrm{H} & -1.64926700 & -3.94791200 & -2.90949500 \\ \mathrm{C} & -2.98883600 & -7.56678600 & -2.60208900 \\ \mathrm{H} & -2.56231400 & -7.39111300 & -0.49812700 \\ \mathrm{C} & -2.97362500 & -6.94289800 & -3.85376900 \\ \mathrm{H} & -2.47492100 & -5.12910400 & -4.91111200 \\ \mathrm{H} & -3.37085300 & -8.57981100 & -2.50294300 \\ \mathrm{H} & 3.75329400 & -0.57585800 & -0.33835200 \\ \mathrm{H} & 1.97894500 & -1.43336900 & -0.41968200 \\ \mathrm{H} & -3.83458300 & 0.53774500 & -0.42937200 \\ \mathrm{H} & -5.99489600 & -0.60030600 & -0.43205100 \\ \mathrm{H} & -6.10544100 & -3.09684800 & -0.41746800 \\ \mathrm{H} & -4.01696700 & -4.41560300 & -0.42858800 \\ \mathrm{H} & -2.05880300 & 1.39542300 & -0.46407100 \\ \mathrm{H} & 3.35833300 & 7.44292700 & -4.62647600 \\ \mathrm{H} & -0.66599700 & 7.12366900 & 3.91884200 \\ \mathrm{H} & -4.15507200 & 6.03773400 & -0.37896300 \\ \mathrm{H} & 4.07281100 & -6.07596800 & -0.30095200 \\ \mathrm{H} & 0.48145800 & -7.17818000 & 3.90619100 \\ \mathrm{H} & -3.33481100 & -7.46480100 & -4.73518700 \\ & & & \end{array}$

\section{Cartesian Coordinate for Optimized Structure of 16'}

SCF Done: E $($ RB3LYP $)=-2189.09285420$ (hartree)

Zero-point correction $=0.737950$ (hartree)

Thermal correction to Energy $=0.779438$ (hartree)

Thermal correction to Enthalpy $=0.780382$ (hartree)

Thermal correction to Gibbs Free Energy $=0.660274$ (hartree)

The number of imaginary frequencies: 0

$\begin{array}{lrrr}\mathrm{C} & -5.06716000 & -1.16544500 & -0.37046300 \\ \mathrm{C} & -3.84479400 & -0.56260000 & -0.42490500 \\ \mathrm{C} & -2.63324400 & -1.31257500 & -0.31351600 \\ \mathrm{C} & -1.34491600 & -0.63171000 & -0.32008200 \\ \mathrm{C} & -1.19841800 & 0.75889800 & -0.31251800 \\ \mathrm{C} & 0.05979400 & 1.37777900 & -0.28993900 \\ \mathrm{C} & 0.17276500 & 2.79543500 & -0.33863400 \\ \mathrm{C} & 1.26158100 & 0.59315300 & -0.28667400 \\ \mathrm{C} & 1.38062700 & 3.43380000 & -0.27818200 \\ \mathrm{C} & -0.14335200 & -1.41635800 & -0.29744600\end{array}$




\begin{tabular}{|c|c|c|c|}
\hline $\mathrm{C}$ & 1.11501200 & -0.79745900 & -0.28765000 \\
\hline $\mathrm{C}$ & 2.54935500 & 1.27390900 & -0.24680500 \\
\hline $\mathrm{C}$ & 2.61774300 & 2.68730900 & -0.12184700 \\
\hline $\mathrm{C}$ & 3.76325500 & 0.52433200 & -0.33237000 \\
\hline $\mathrm{C}$ & 3.90496100 & 3.31120500 & 0.14161100 \\
\hline $\mathrm{C}$ & 4.98394400 & 1.12692300 & -0.24647800 \\
\hline $\mathrm{C}$ & 5.09285300 & 2.51896200 & 0.03140800 \\
\hline $\mathrm{H}$ & 5.89574000 & 0.54287300 & -0.34542900 \\
\hline $\mathrm{C}$ & -2.70453800 & -2.72646400 & -0.19578900 \\
\hline $\mathrm{C}$ & -1.46396600 & -3.47235200 & -0.32519400 \\
\hline $\mathrm{C}$ & -3.99766000 & -3.35136700 & 0.03430000 \\
\hline $\mathrm{C}$ & -0.25503500 & -2.83381500 & -0.35408400 \\
\hline $\mathrm{C}$ & -5.18261400 & -2.55859400 & -0.10090400 \\
\hline $\mathrm{N}$ & 1.38486300 & 4.84784100 & -0.47026700 \\
\hline $\mathrm{N}$ & -1.46348200 & -4.88564900 & -0.52282500 \\
\hline $\mathrm{C}$ & 0.60993600 & 5.65744300 & 0.39133900 \\
\hline $\mathrm{C}$ & -0.11143400 & 6.76203800 & -0.09135100 \\
\hline $\mathrm{C}$ & 0.54971200 & 5.34719300 & 1.76102100 \\
\hline $\mathrm{C}$ & -0.86570100 & 7.54217900 & 0.78443200 \\
\hline $\mathrm{H}$ & -0.07099800 & 7.00173800 & -1.14853900 \\
\hline $\mathrm{C}$ & -0.22020800 & 6.12431200 & 2.62474200 \\
\hline $\mathrm{H}$ & 1.10366000 & 4.49100300 & 2.13327300 \\
\hline $\mathrm{C}$ & -0.92950600 & 7.22972900 & 2.14561200 \\
\hline $\mathrm{H}$ & -1.41913400 & 8.39253400 & 0.39412000 \\
\hline $\mathrm{H}$ & -0.25571900 & 5.86872900 & 3.68061700 \\
\hline $\mathrm{C}$ & 2.03511100 & 5.36664900 & -1.61442400 \\
\hline $\mathrm{C}$ & 2.18594400 & 4.57524900 & -2.76403400 \\
\hline $\mathrm{C}$ & 2.58586100 & 6.65992200 & -1.59935900 \\
\hline $\mathrm{C}$ & 2.87422600 & 5.07082300 & -3.87141600 \\
\hline $\mathrm{H}$ & 1.76786300 & 3.57466900 & -2.78267400 \\
\hline $\mathrm{C}$ & 3.25504000 & 7.15151900 & -2.71753800 \\
\hline $\mathrm{H}$ & 2.48415000 & 7.27018600 & -0.70835800 \\
\hline $\mathrm{C}$ & 3.40879100 & 6.36105600 & -3.86080000 \\
\hline $\mathrm{H}$ & 2.98313800 & 4.44249900 & -4.75167600 \\
\hline $\mathrm{H}$ & 3.67365000 & 8.15427500 & -2.68712100 \\
\hline $\mathrm{C}$ & -0.70974000 & -5.69868100 & 0.35417800 \\
\hline $\mathrm{C}$ & 0.02266700 & -6.80184600 & -0.11500600 \\
\hline $\mathrm{C}$ & -0.68232000 & -5.39338400 & 1.72602800 \\
\hline $\mathrm{C}$ & 0.75540000 & -7.58544800 & 0.77583800 \\
\hline $\mathrm{H}$ & 0.00763100 & -7.03773600 & -1.17371800 \\
\hline $\mathrm{C}$ & 0.06633400 & -6.17394800 & 2.60519300 \\
\hline $\mathrm{H}$ & -1.24474400 & -4.53829400 & 2.08795400 \\
\hline $\mathrm{C}$ & 0.78656700 & -7.27794600 & 2.13928000 \\
\hline $\mathrm{H}$ & 1.31777800 & -8.43461500 & 0.39586100 \\
\hline $\mathrm{H}$ & 0.07654500 & -5.92217000 & 3.66252900 \\
\hline
\end{tabular}




$\begin{array}{lrrr}\mathrm{C} & -2.08543300 & -5.39979300 & -1.68468800 \\ \mathrm{C} & -2.20757100 & -4.60389100 & -2.83458900 \\ \mathrm{C} & -2.63675300 & -6.69290100 & -1.68823400 \\ \mathrm{C} & -2.86836200 & -5.09493300 & -3.96058200 \\ \mathrm{H} & -1.78879400 & -3.60343600 & -2.83899000 \\ \mathrm{C} & -3.27818400 & -7.17993900 & -2.82452700 \\ \mathrm{H} & -2.55725800 & -7.30662700 & -0.79735300 \\ \mathrm{C} & -3.40336500 & -6.38501100 & -3.96818000 \\ \mathrm{H} & -2.95529100 & -4.46318400 & -4.84083600 \\ \mathrm{H} & -3.69770200 & -8.18264500 & -2.80835800 \\ \mathrm{H} & 3.71963300 & -0.54489500 & -0.50073300 \\ \mathrm{H} & 1.98097900 & -1.44968000 & -0.26051700 \\ \mathrm{H} & -3.79724300 & 0.50730200 & -0.58781500 \\ \mathrm{H} & -5.97636300 & -0.58092300 & -0.48862900 \\ \mathrm{H} & -2.06480600 & 1.41106400 & -0.30381100 \\ \mathrm{H} & 3.93948300 & 6.74458000 & -4.72758200 \\ \mathrm{H} & -1.52304700 & 7.83794200 & 2.82218500 \\ \mathrm{H} & 1.36346200 & -7.88884800 & 2.82772300 \\ \mathrm{H} & -3.91255200 & -6.76499100 & -4.84930500 \\ \mathrm{H} & -0.72651300 & 3.39219800 & -0.46254700 \\ \mathrm{H} & 0.64699900 & -3.43014600 & -0.45849800 \\ \mathrm{C} & 6.36515600 & 3.10282000 & 0.24870000 \\ \mathrm{C} & 4.06690000 & 4.65433100 & 0.57910500 \\ \mathrm{C} & 5.31741400 & 5.19273400 & 0.81306100 \\ \mathrm{H} & 5.39626400 & 6.22181500 & 1.15302600 \\ \mathrm{C} & 6.48453500 & 4.42409100 & 0.62322900 \\ \mathrm{H} & 7.46368700 & 4.86291500 & 0.79503400 \\ \mathrm{H} & 3.19731100 & 5.26513500 & 0.76569200 \\ \mathrm{H} & 7.24571100 & 2.47456600 & 0.13550000 \\ \mathrm{C} & -6.45971800 & -3.14320700 & 0.08377300 \\ \mathrm{C} & -4.16996000 & -4.69628000 & 0.46227400 \\ \mathrm{C} & -5.42567600 & -5.23549600 & 0.66432400 \\ \mathrm{H} & -5.51256800 & -6.26595700 & 0.99809600 \\ \mathrm{C} & -6.58796400 & -4.46596300 & 0.45002900 \\ \mathrm{H} & -7.57091900 & -4.90536900 & 0.59680300 \\ \mathrm{H} & -3.30507000 & -5.30798200 & 0.66690600 \\ & -7.33733400 & -2.51440500 & -0.04772700\end{array}$




\section{References and Notes}

(1) Yu, M.; Wang, M.; Chen, X.; Hong, B.; Zhang, X.; Cheng, C. Synthesis of OLED Materials of Several Triarylamines by Palladium Catalysts and Their Light Emitting Property. J. Chem. Res. 2005, 2005, 558-560. DOI: 10.3184/030823405774308961.

(2) Frisch, M. J.; Trucks, G. W.; Schlegel, H. B.; Scuseria, G. E.; Robb, M. A.; Cheeseman, J. R.; Scalmani, G.; Barone, V.; Mennucci, B.; Petersson, G. A.; Nakatsuji, H.; Caricato, M.; Li, X.; Hratchian, H. P.; Izmaylov, A. F.; Bloino, J.; Zheng, G.; Sonnenberg, J. L.; Had, M.; Fox, D. J. Gaussian 09 C.01. Gaussian, Inc. Wallingford CT. 2010.

(3) Becke, A. D. Density-functional Thermochemistry. III. The Role of Exact Exchange. J. Chem. Phys. 1993, 98, 5648-5652. DOI: 10.1063/1.464913.

(4) Lee, C.; Yang, W.; Parr, R. G. Development of the Colle-Salvetti Correlation-Energy Formula into a Functional of the Electron Density. Phys. Rev. B 1988, 37, 785-789. DOI: 10.1103/PhysRevB.37.785.

(5) Ditchfield, R.; Hehre, W. J.; Pople, J. A. Self-Consistent Molecular-Orbital Methods. IX. An Extended Gaussian-Type Basis for Molecular-Orbital Studies of Organic Molecules. J. Chem. Phys. 1971, 54, 724. DOI: 10.1063/1.1674902.

(6) Hehre, W. J.; Ditchfield, R.; Pople, J. A. Self-Consistent Molecular Orbital Methods. XII. Further Extensions of Gaussian-Type Basis Sets for Use in Molecular Orbital Studies of Organic Molecules. J. Chem. Phys. 1972, 56, 2257. DOI: 10.1063/1.1677527.

(7) Grimme, S. Semiempirical GGA-Type Density Functional Constructed with a Long-Range Dispersion Correction. J. Comput. Chem. 2006, 27, 1787-1799. DOI: 10.1002/jcc.20495. 
7. Copies of ${ }^{1} \mathbf{H},{ }^{13} \mathrm{C}\left\{{ }^{1} \mathbf{H}\right\},{ }^{19} \mathrm{~F}\left\{{ }^{1} \mathrm{H}\right\}$, and ${ }^{31} \mathbf{P}\left\{{ }^{1} \mathrm{H}\right\}$ NMR Charts

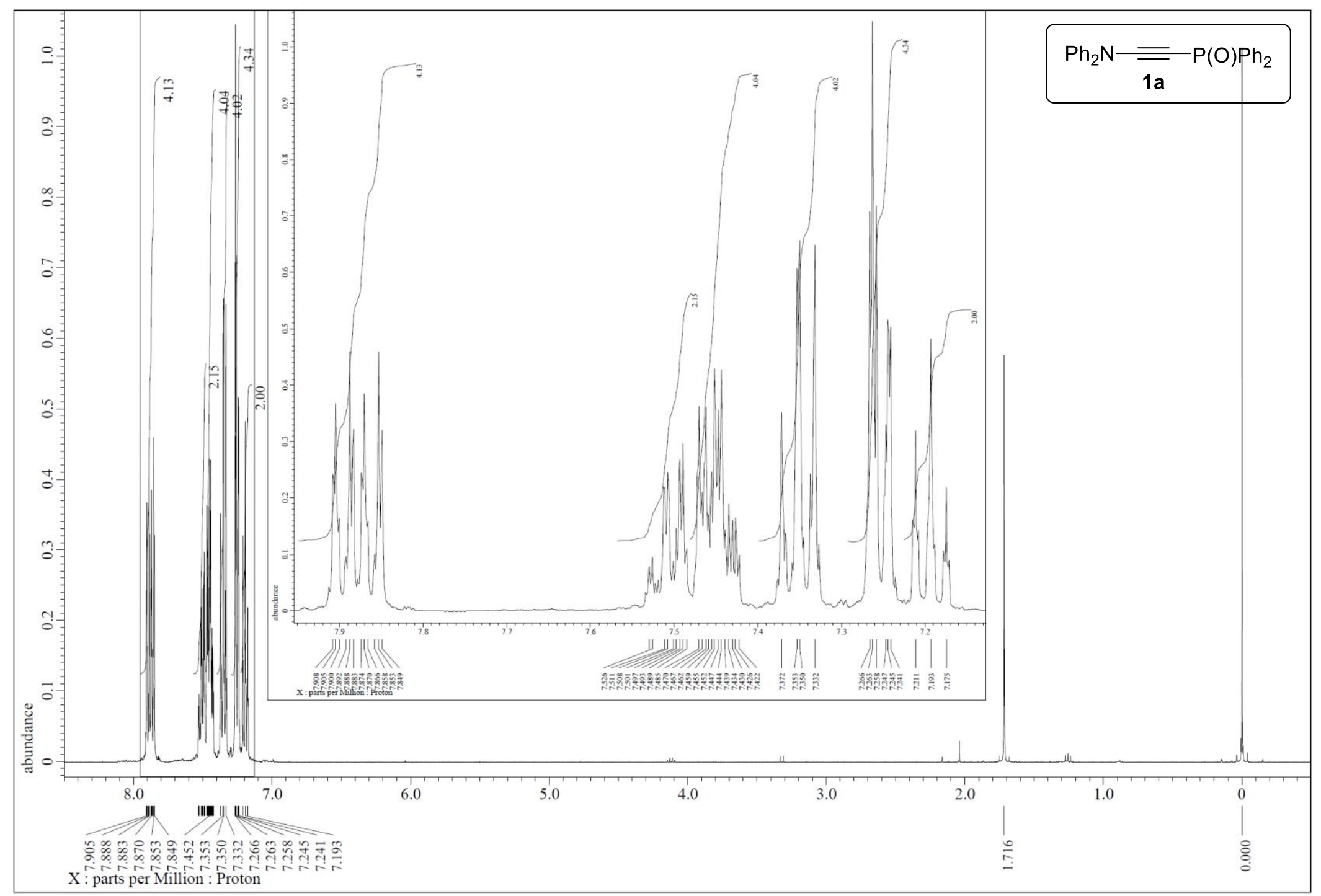

Figure S8. ${ }^{1} \mathrm{H}$ NMR $(400 \mathrm{MHz})$ spectrum of $\mathbf{1 a}\left(\mathrm{CDCl}_{3}, \mathrm{rt}\right)$ 


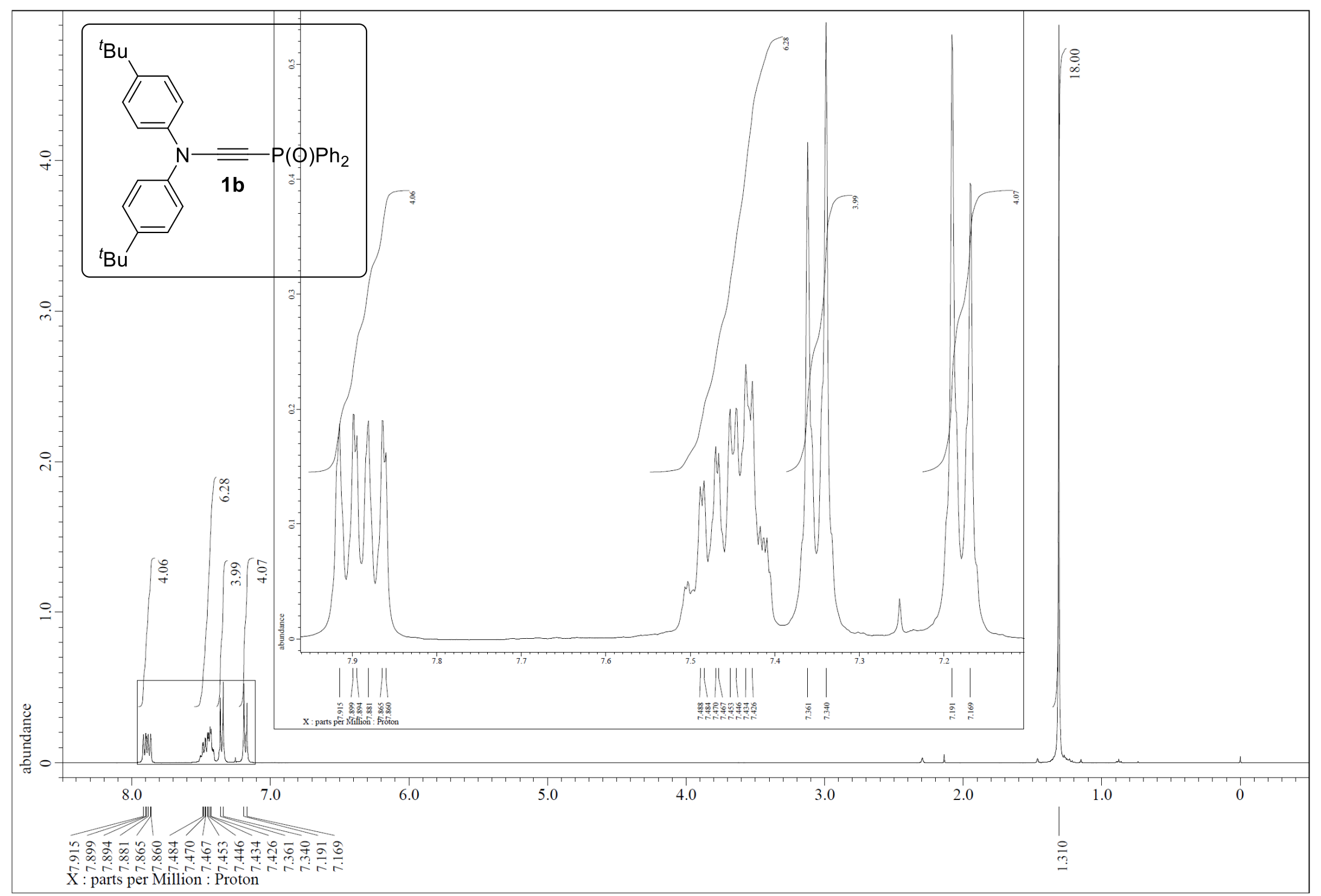

Figure S9. ${ }^{1} \mathrm{H}$ NMR $(400 \mathrm{MHz})$ spectrum of $\mathbf{1 b}\left(\mathrm{CDCl}_{3}, \mathrm{rt}\right)$ 


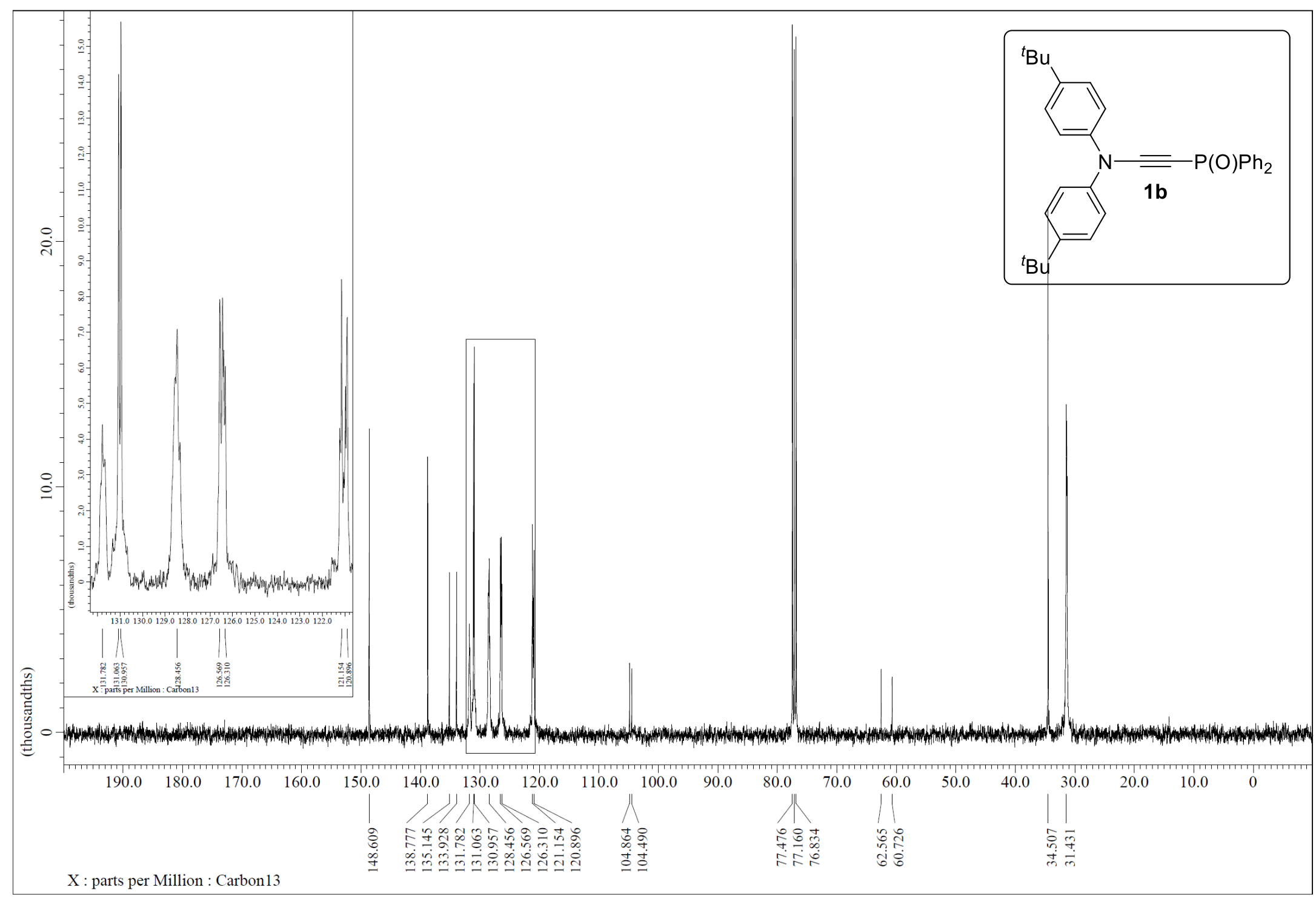

Figure S10. ${ }^{13} \mathrm{C}\left\{{ }^{1} \mathrm{H}\right\}$ NMR $(101 \mathrm{MHz})$ spectrum of $\mathbf{1 b}\left(\mathrm{CDCl}_{3}, \mathrm{rt}\right)$ 


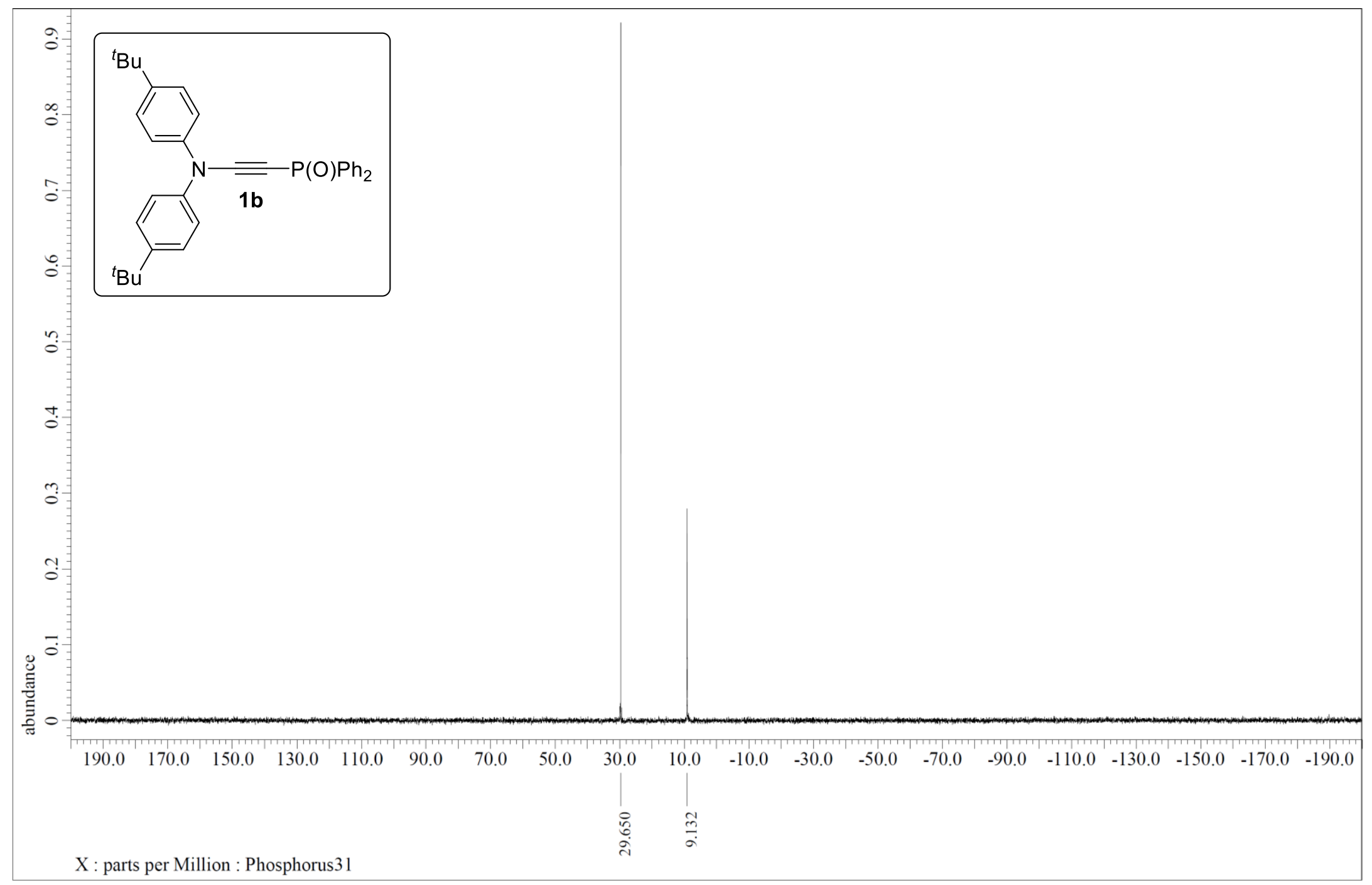

Figure S11. ${ }^{31} \mathrm{P}\left\{{ }^{1} \mathrm{H}\right\}$ NMR $(162 \mathrm{MHz})$ spectrum of $\mathbf{1 b}\left(\mathrm{CDCl}_{3}, \mathrm{rt}\right)$ 


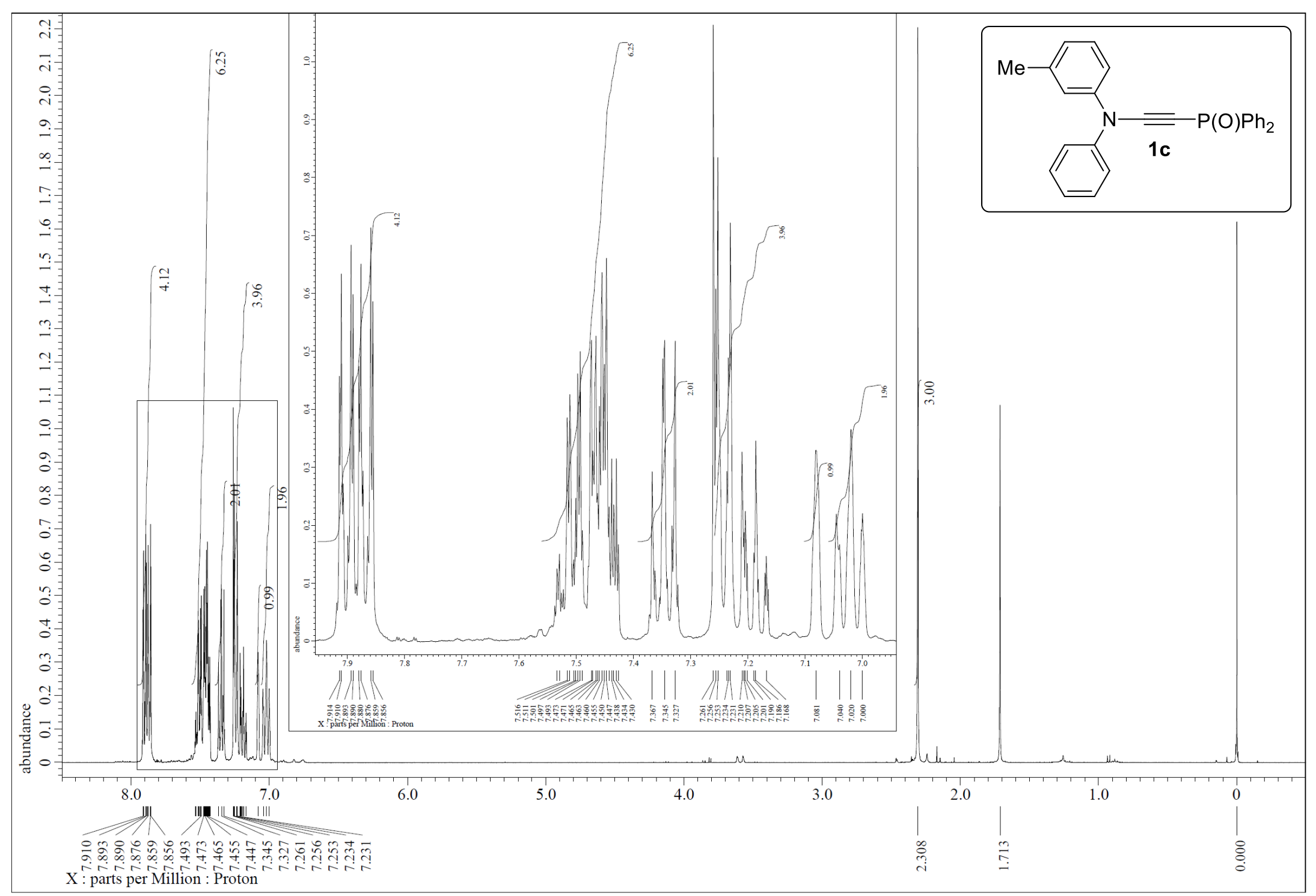

Figure S12. ${ }^{1} \mathrm{H}$ NMR $(400 \mathrm{MHz})$ spectrum of $\mathbf{1 c}\left(\mathrm{CDCl}_{3}, \mathrm{rt}\right)$ 


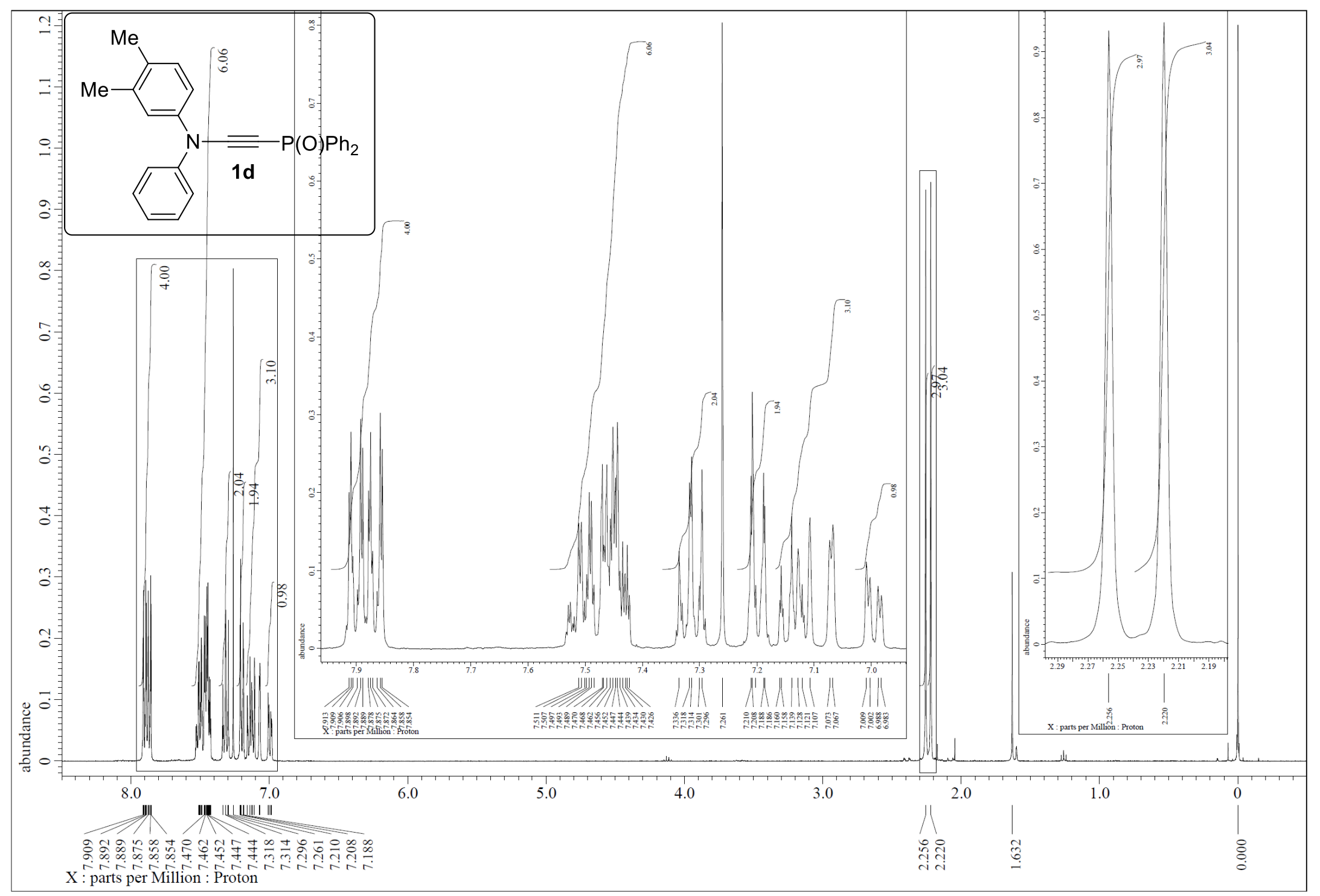

Figure S13. ${ }^{1} \mathrm{H}$ NMR $(400 \mathrm{MHz})$ spectrum of $\mathbf{1 d}\left(\mathrm{CDCl}_{3}, \mathrm{rt}\right)$ 


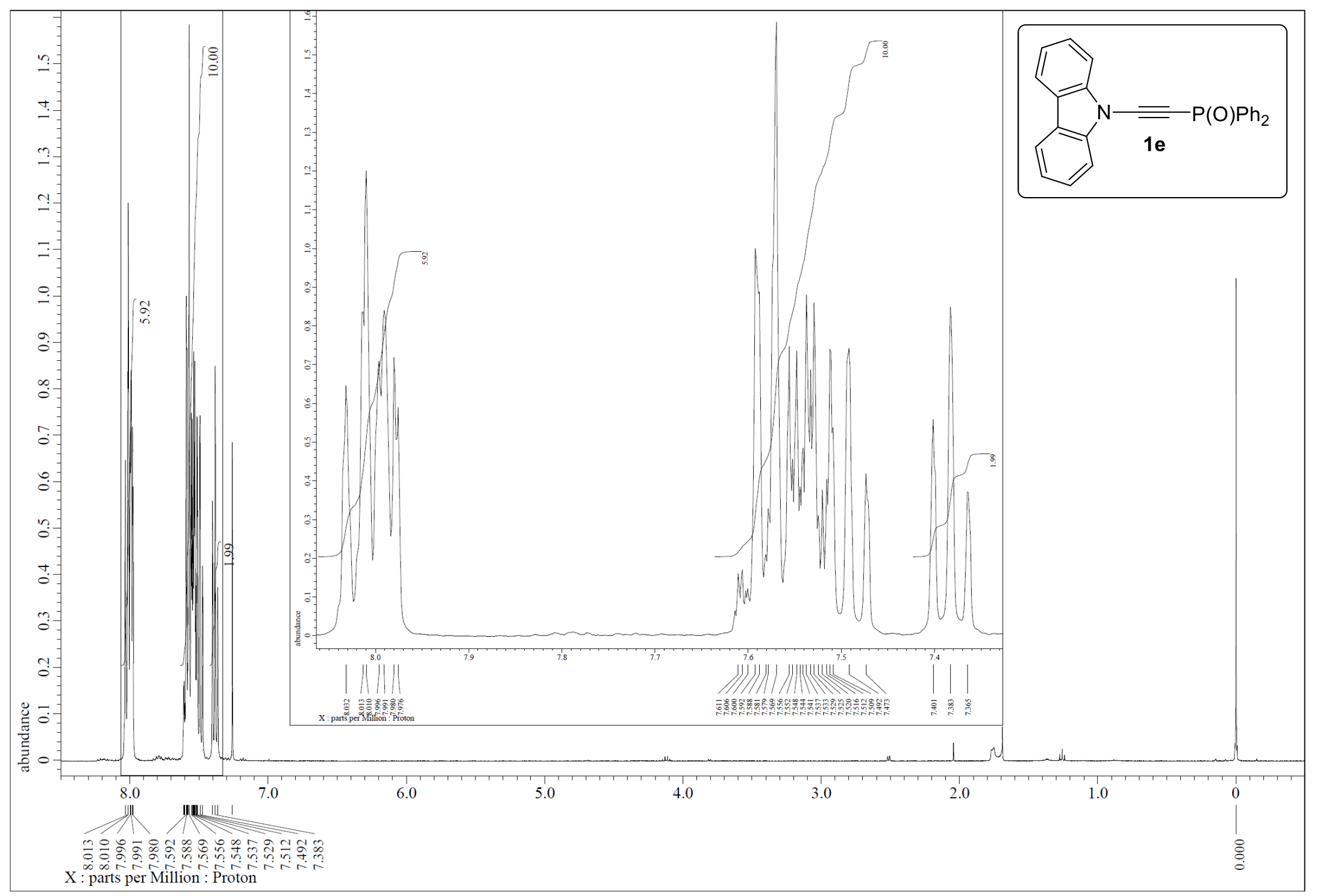

Figure S14. ${ }^{1} \mathrm{H}$ NMR $(400 \mathrm{MHz})$ spectrum of $1 \mathbf{e}\left(\mathrm{CDCl}_{3}, \mathrm{rt}\right)$ 


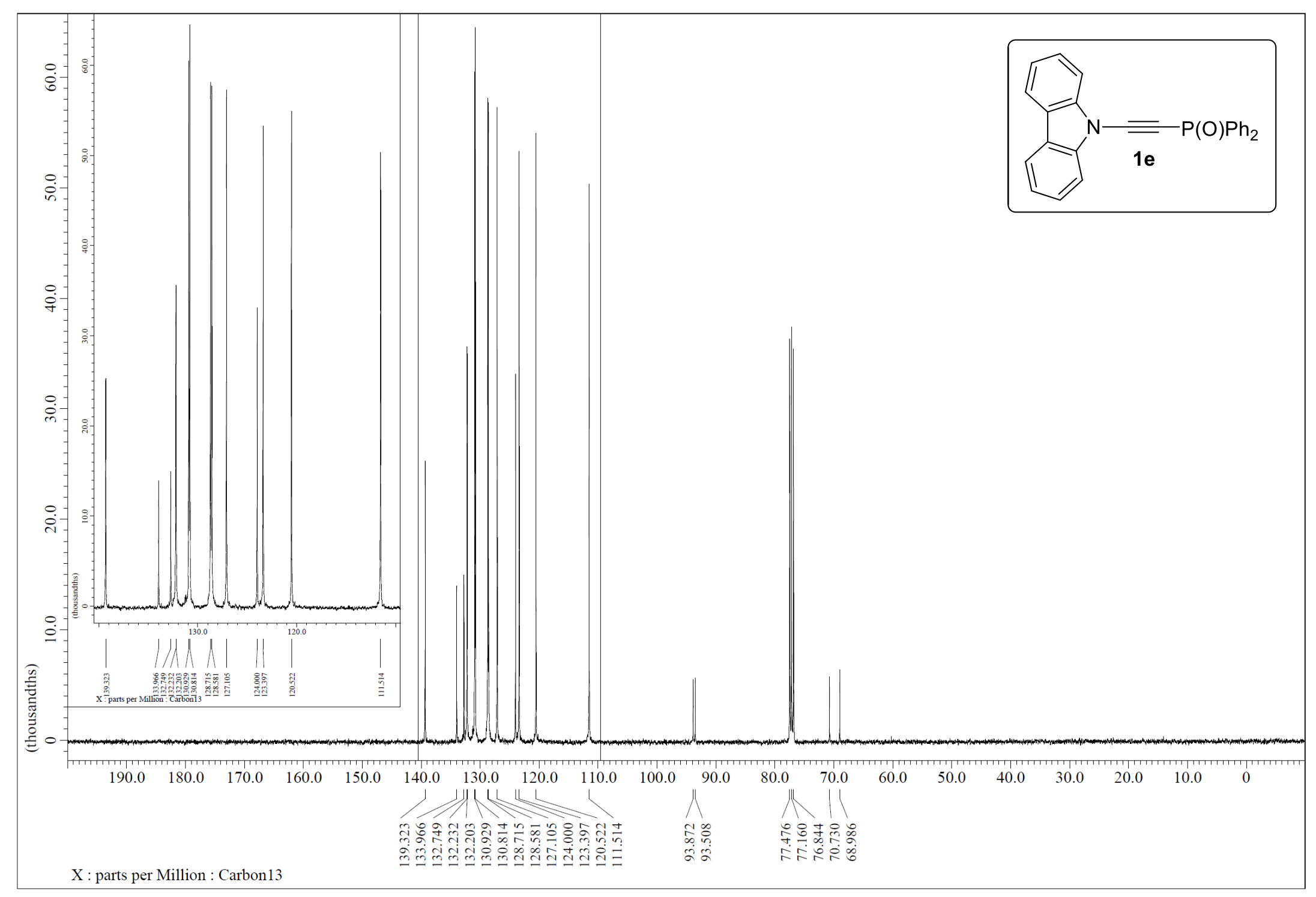

Figure S15. ${ }^{13} \mathrm{C}\left\{{ }^{1} \mathrm{H}\right\}$ NMR $(101 \mathrm{MHz})$ spectrum of $\mathbf{1 e}\left(\mathrm{CDCl}_{3}, \mathrm{rt}\right)$ 


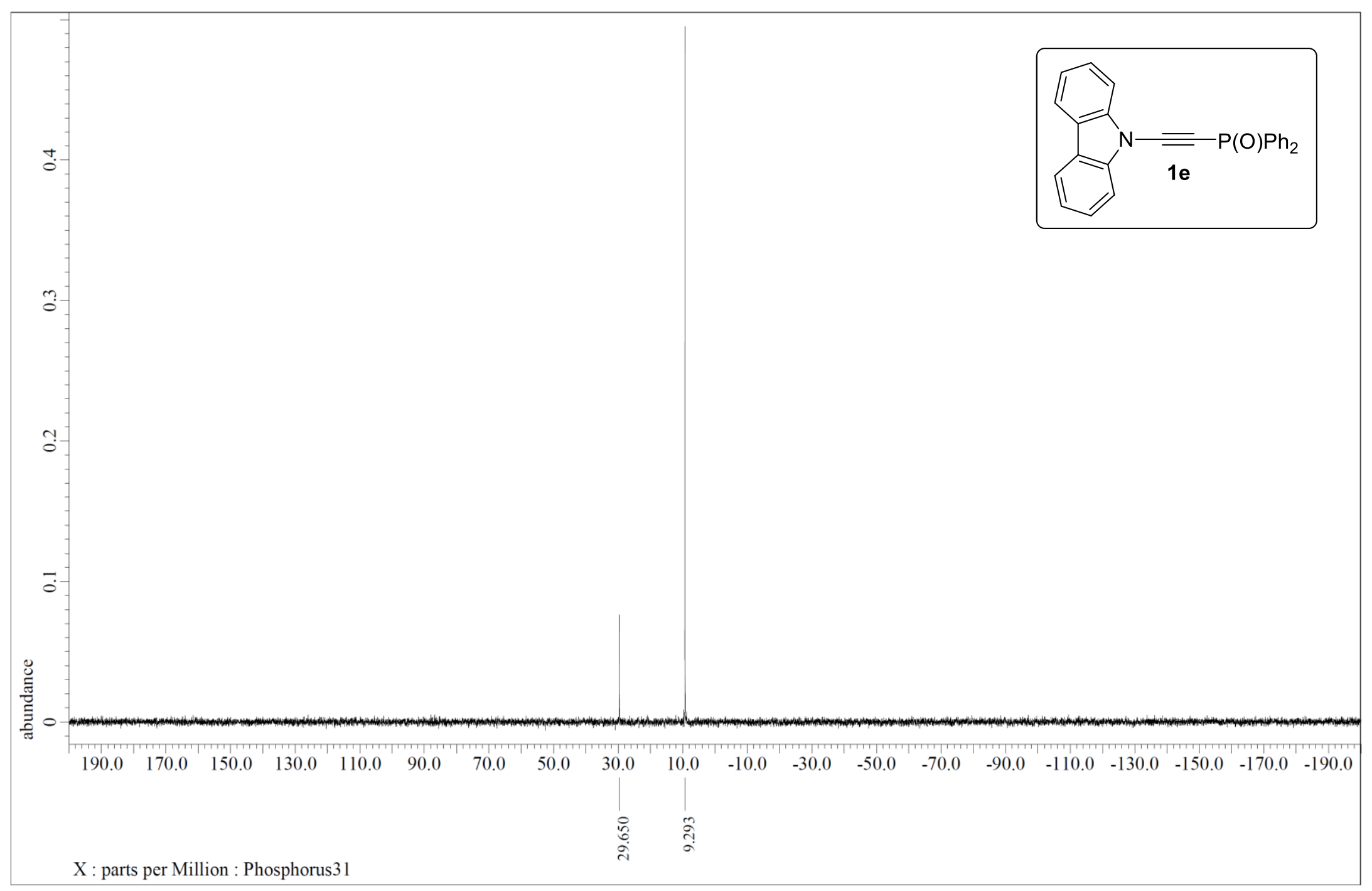

Figure S16. ${ }^{31} \mathrm{P}\left\{{ }^{1} \mathrm{H}\right\}$ NMR (162 MHz) spectrum of $1 \mathrm{e}\left(\mathrm{CDCl}_{3}, \mathrm{rt}\right)$ 


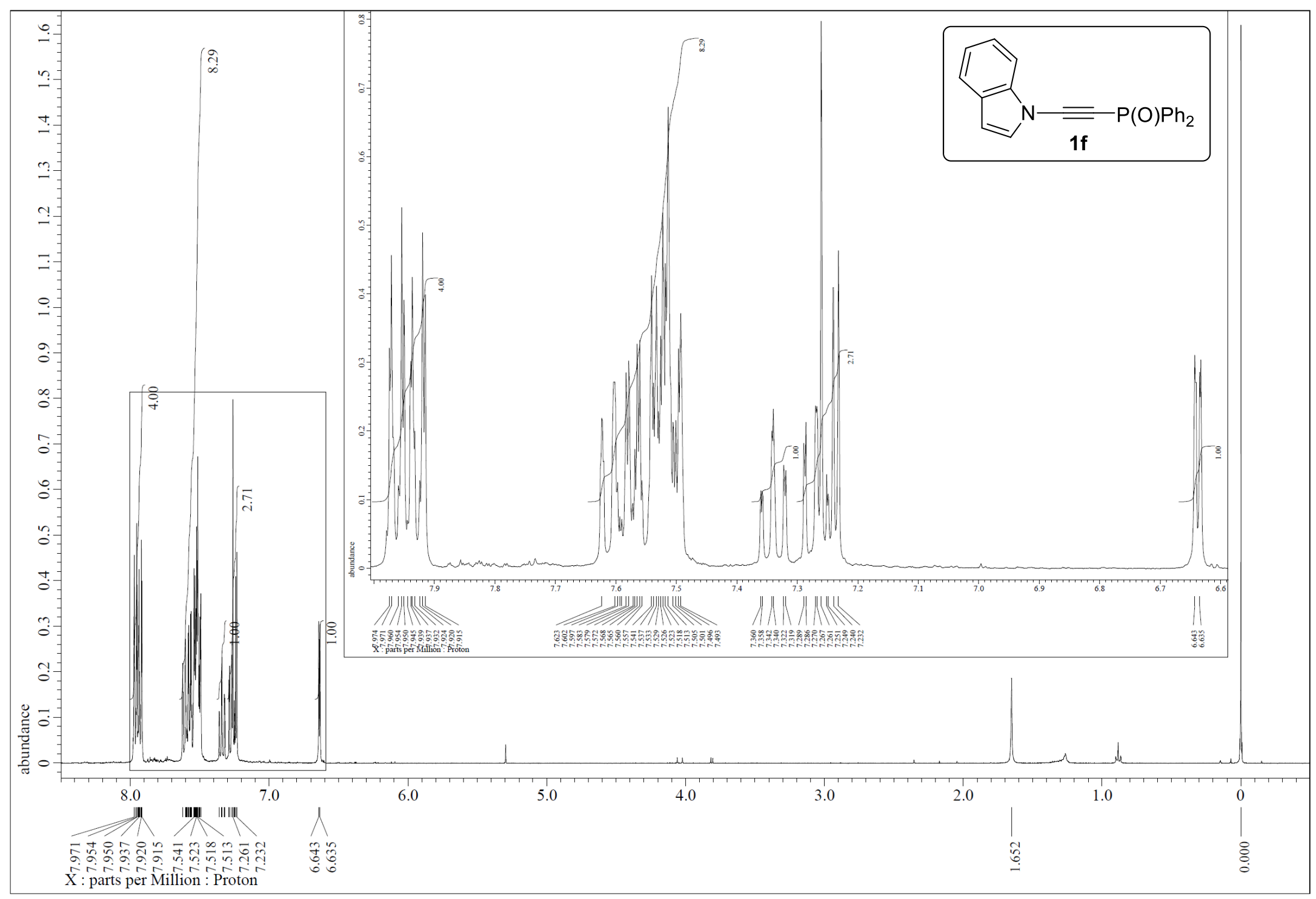

Figure S17. ${ }^{1} \mathrm{H}$ NMR $(400 \mathrm{MHz})$ spectrum of $\mathbf{1 f}\left(\mathrm{CDCl}_{3}, \mathrm{rt}\right)$ 


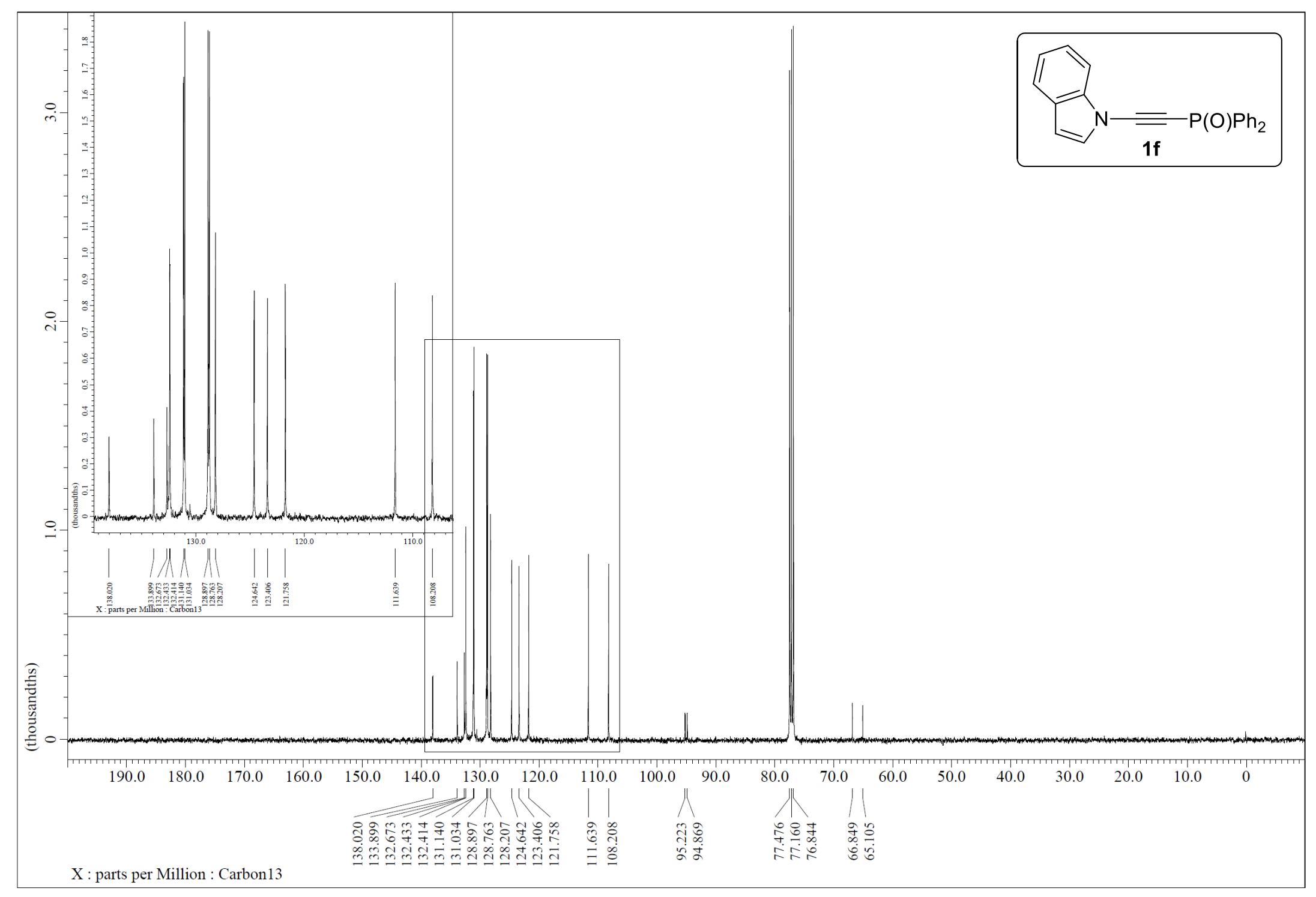

Figure S18. ${ }^{13} \mathrm{C}\left\{{ }^{1} \mathrm{H}\right\} \mathrm{NMR}(101 \mathrm{MHz})$ spectrum of $\mathbf{1 f}\left(\mathrm{CDCl}_{3}, \mathrm{rt}\right)$ 


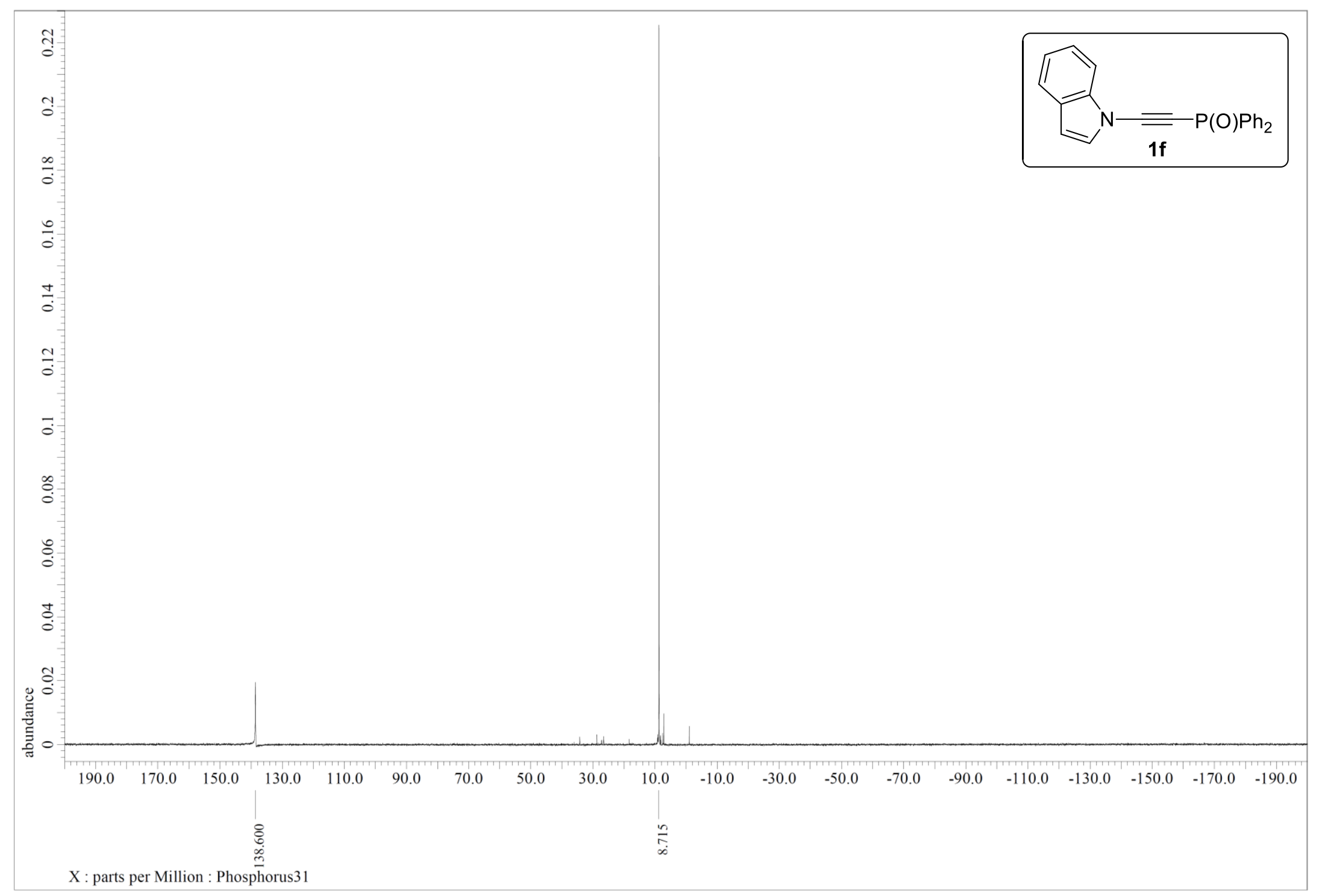

Figure S19. ${ }^{31} \mathrm{P}\left\{{ }^{1} \mathrm{H}\right\} \mathrm{NMR}(162 \mathrm{MHz})$ spectrum of $\mathbf{1 f}\left(\mathrm{CDCl}_{3}, \mathrm{rt}\right)$ 


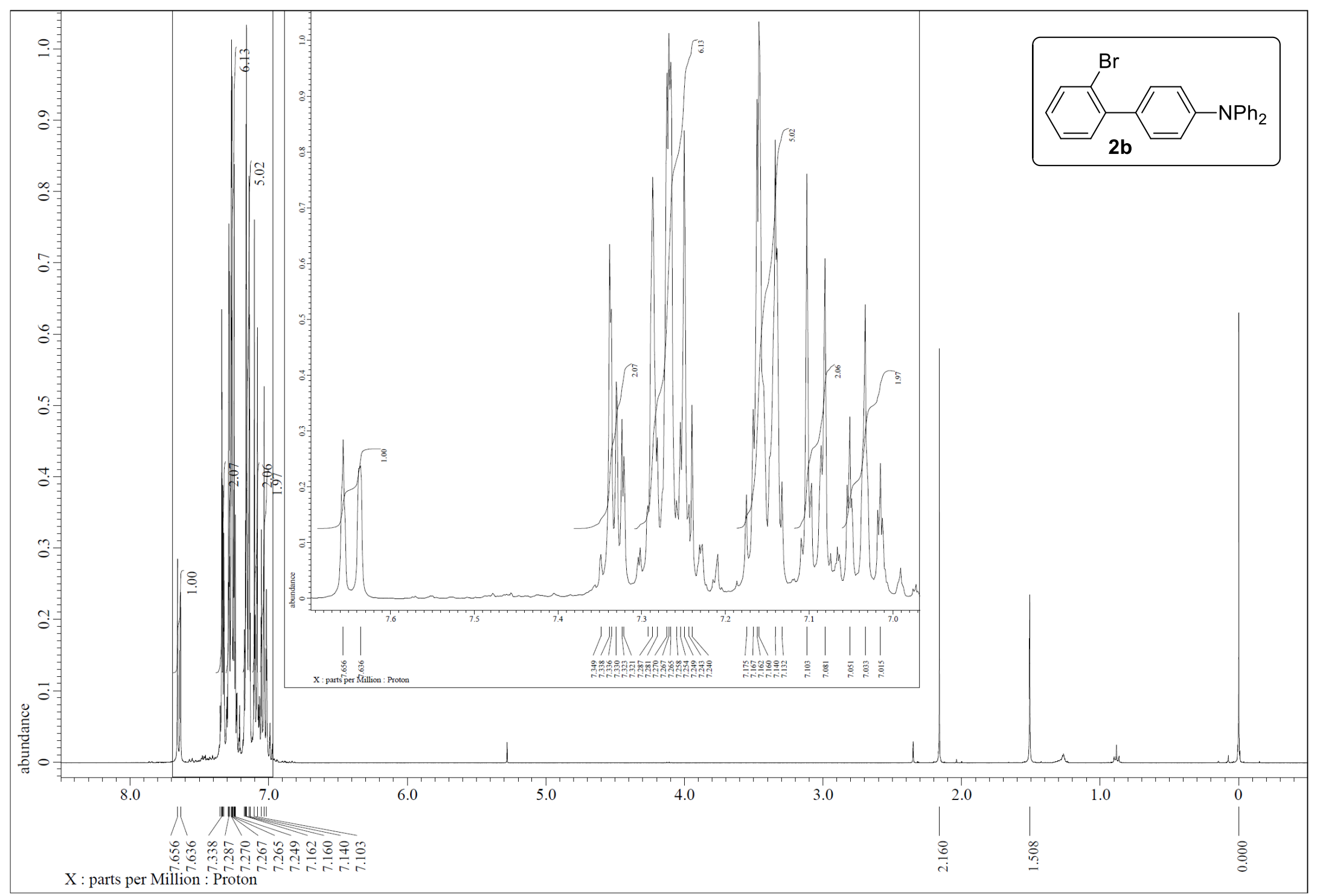

Figure S20. ${ }^{1} \mathrm{H}$ NMR (400 MHz) spectrum of $\mathbf{2 b}\left(\mathrm{CDCl}_{3}, \mathrm{rt}\right)$ 


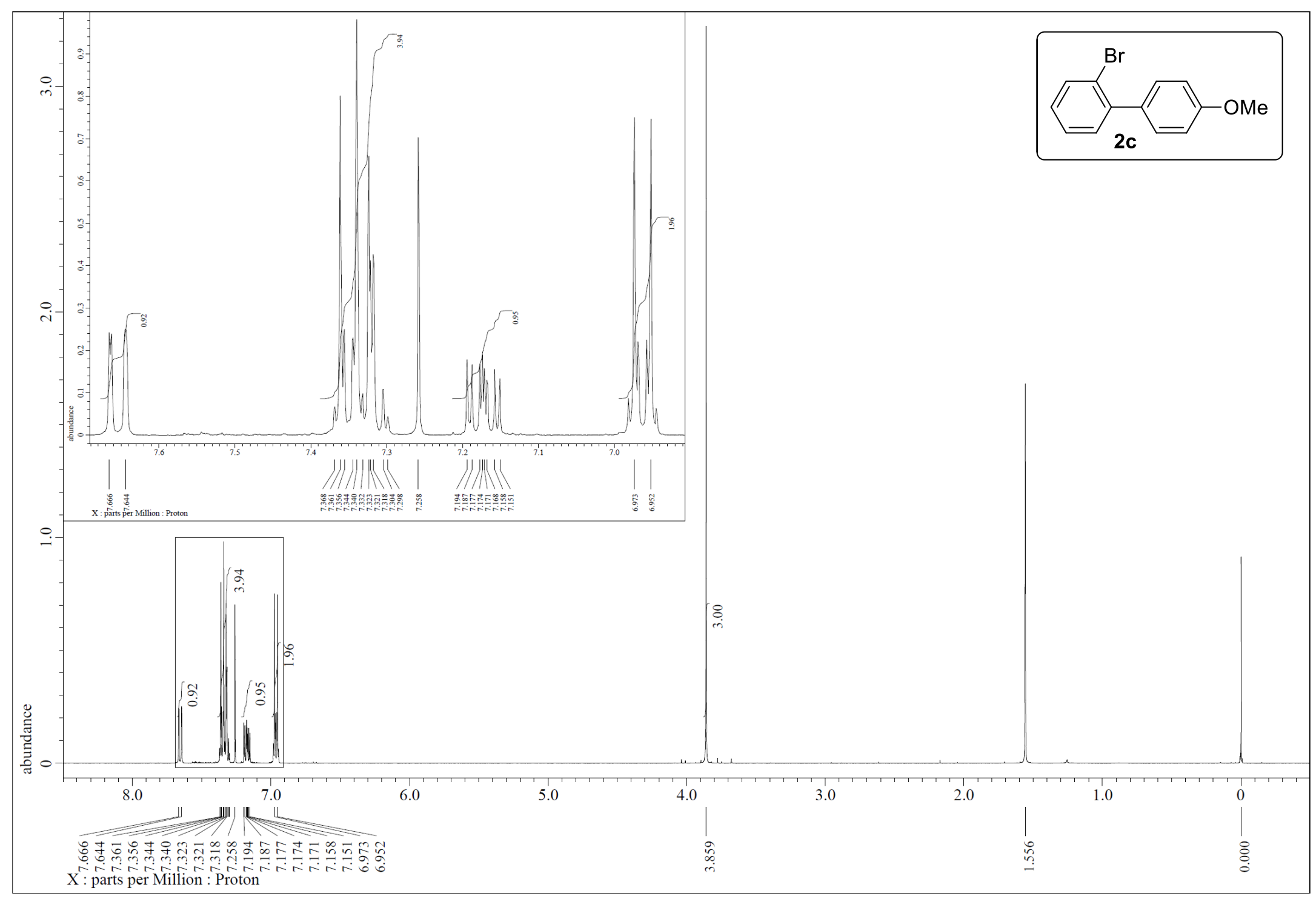

Figure S21. ${ }^{1} \mathrm{H}$ NMR $(400 \mathrm{MHz})$ spectrum of $2 \mathbf{c}\left(\mathrm{CDCl}_{3}, \mathrm{rt}\right)$ 


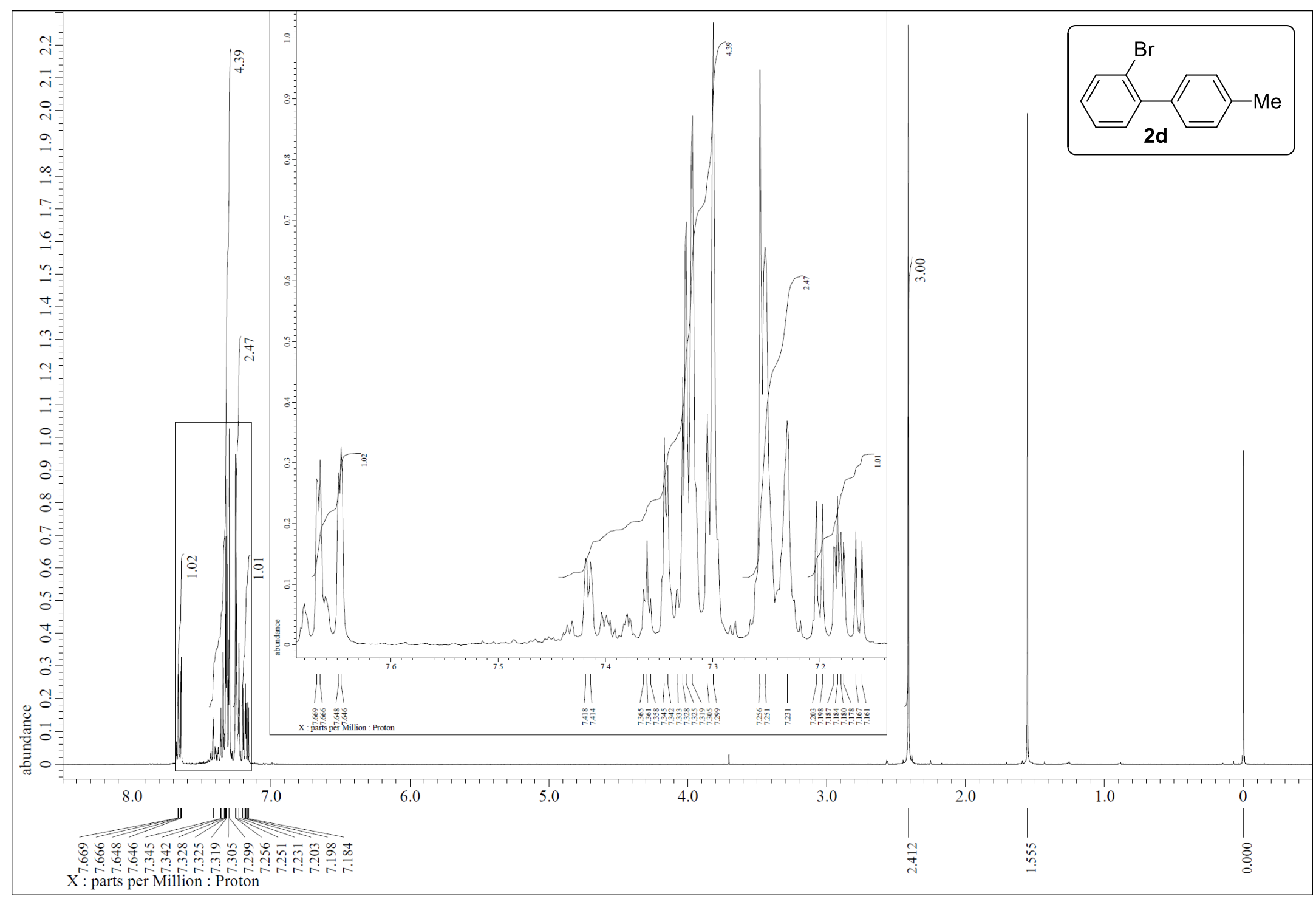

Figure S22. ${ }^{1} \mathrm{H}$ NMR $(400 \mathrm{MHz})$ spectrum of $\mathbf{2 d}\left(\mathrm{CDCl}_{3}, \mathrm{rt}\right)$ 


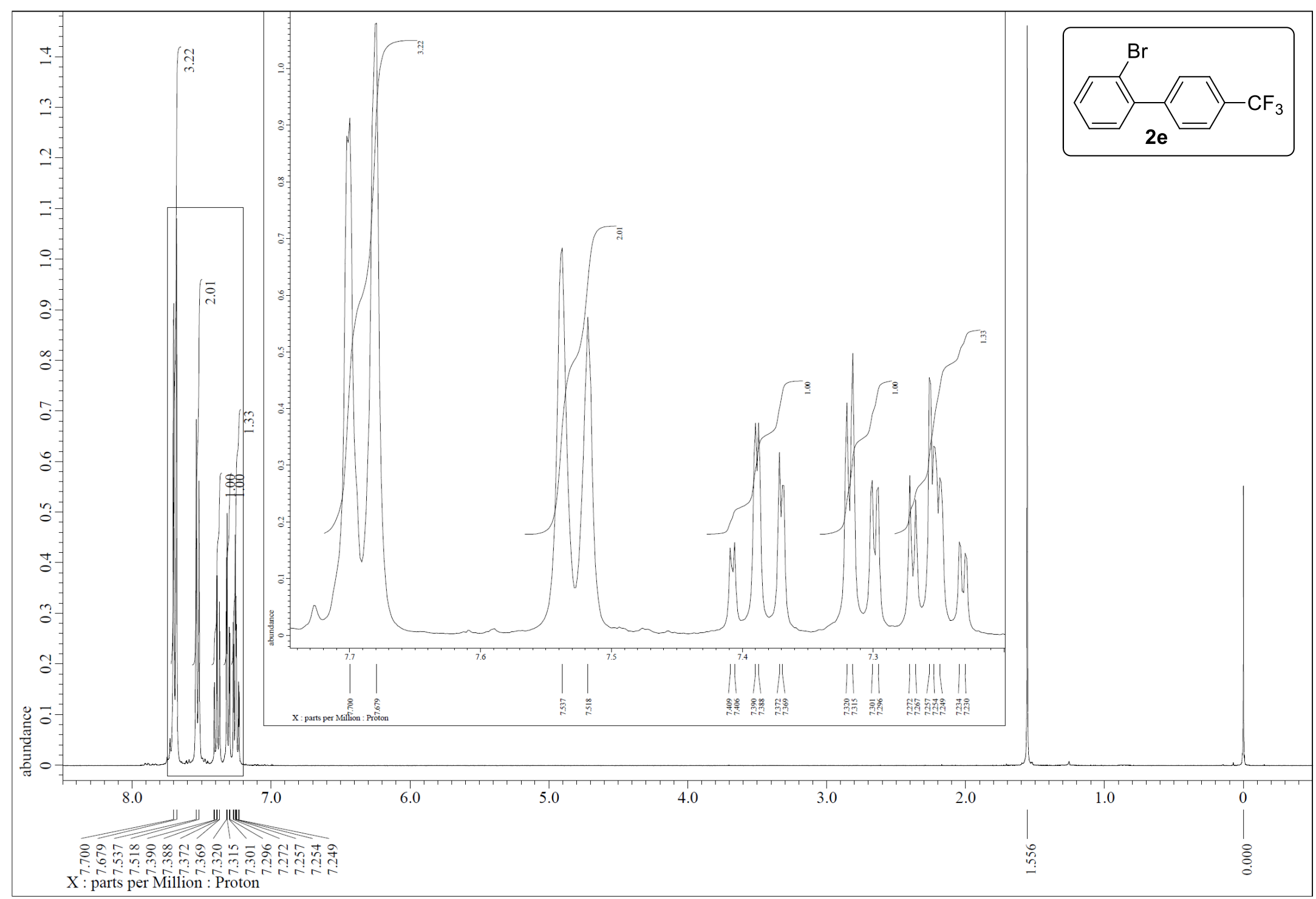

Figure S23. ${ }^{1} \mathrm{H}$ NMR $(400 \mathrm{MHz})$ spectrum of $\mathbf{2 e}\left(\mathrm{CDCl}_{3}, \mathrm{rt}\right)$ 


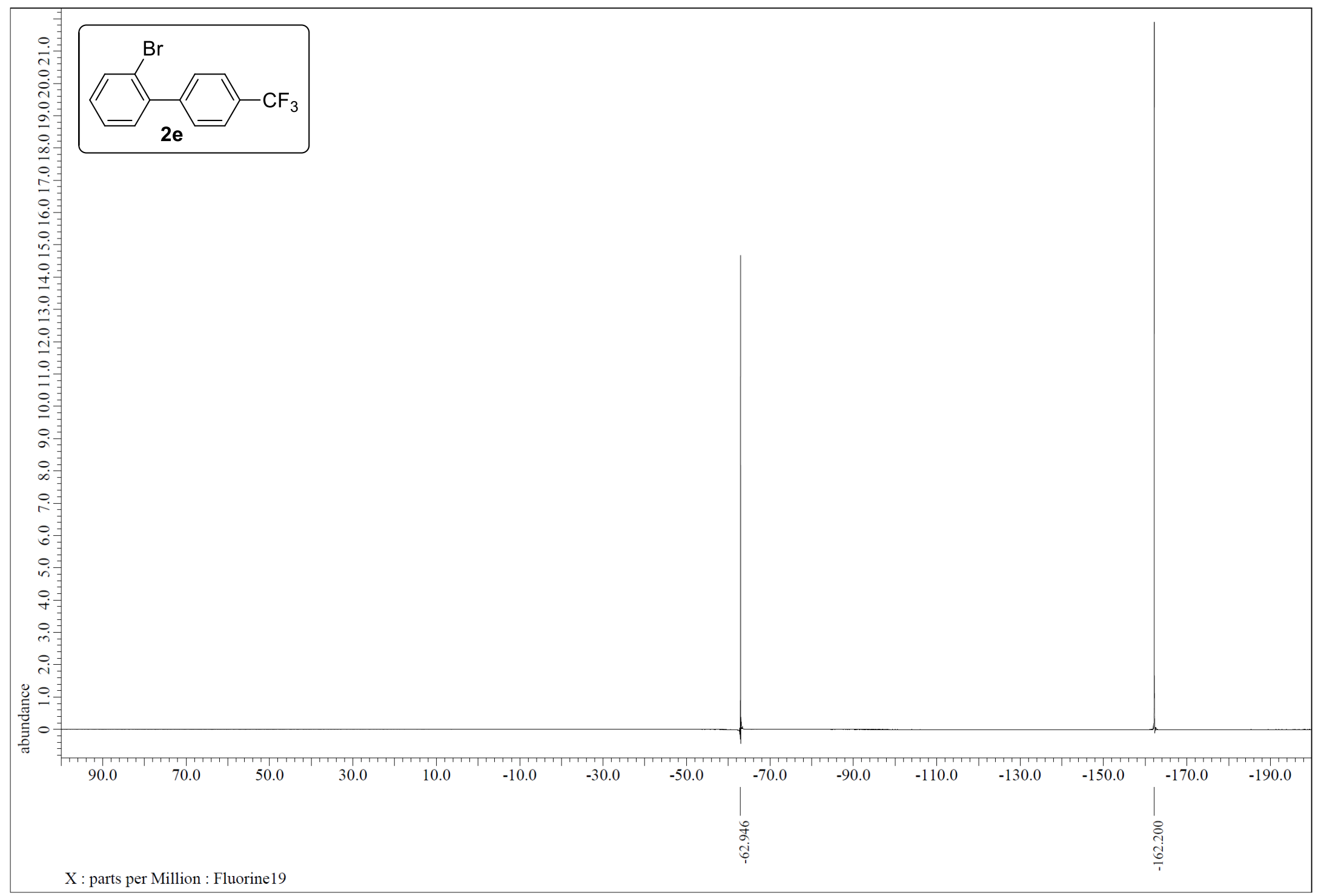

Figure S24. ${ }^{19} \mathrm{~F}\left\{{ }^{1} \mathrm{H}\right\}$ NMR $(376 \mathrm{MHz})$ spectrum of $2 \mathbf{e}\left(\mathrm{CDCl}_{3}, \mathrm{rt}\right)$ 


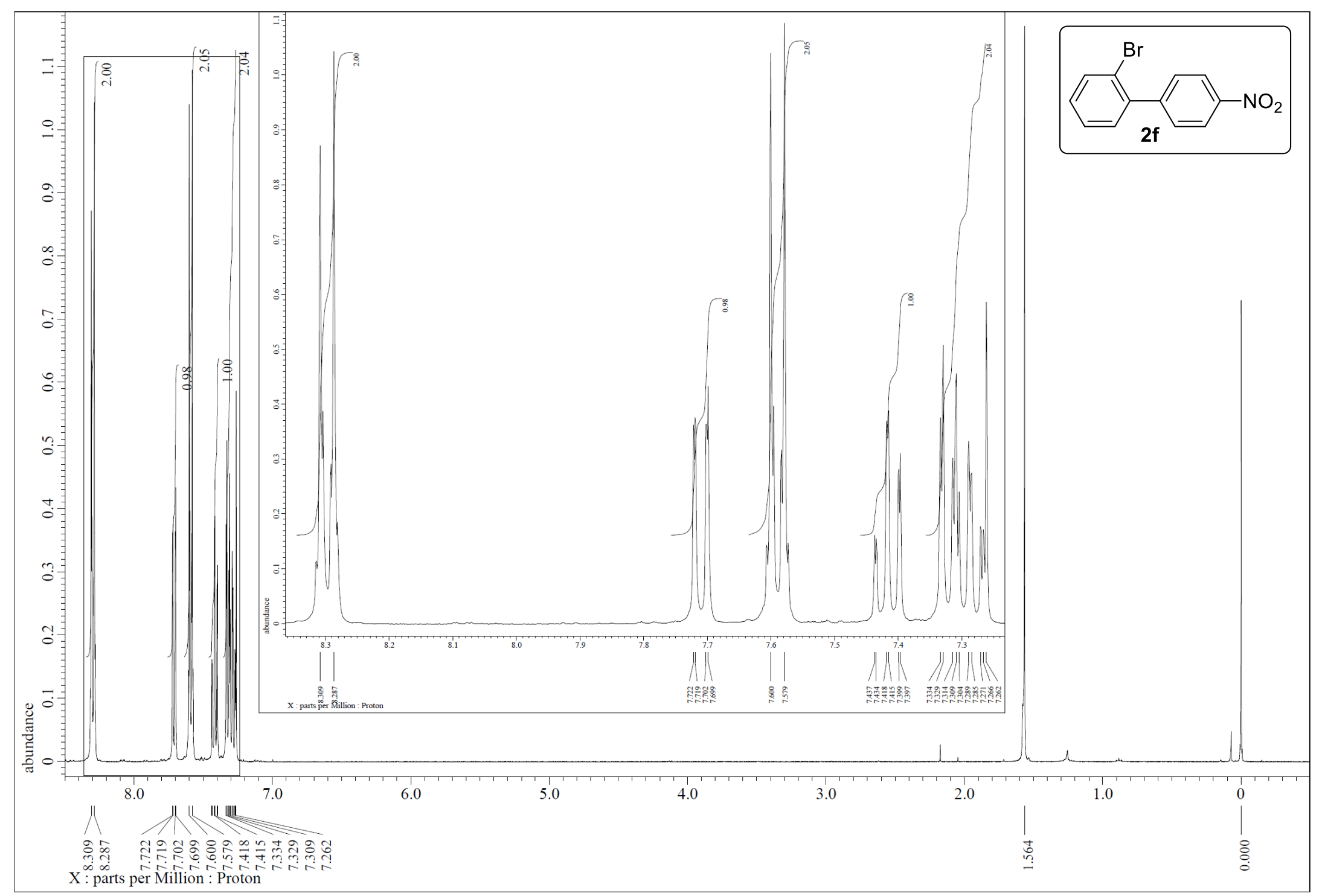

Figure S25. ${ }^{1} \mathrm{H}$ NMR $(400 \mathrm{MHz})$ spectrum of $\mathbf{2 f}\left(\mathrm{CDCl}_{3}, \mathrm{rt}\right)$ 


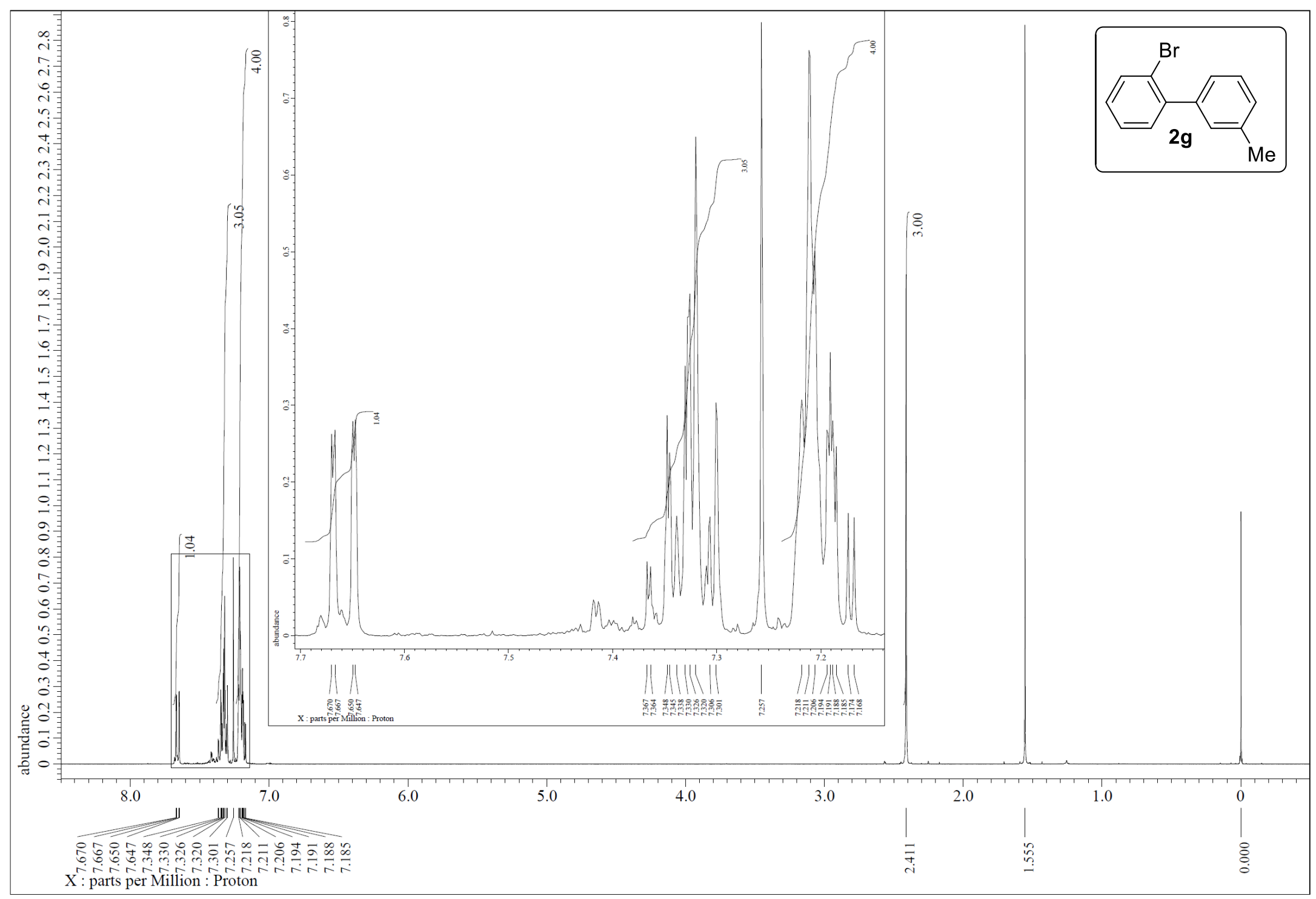

Figure S26. ${ }^{1} \mathrm{H}$ NMR $(400 \mathrm{MHz})$ spectrum of $\mathbf{2 g}\left(\mathrm{CDCl}_{3}, \mathrm{rt}\right)$ 


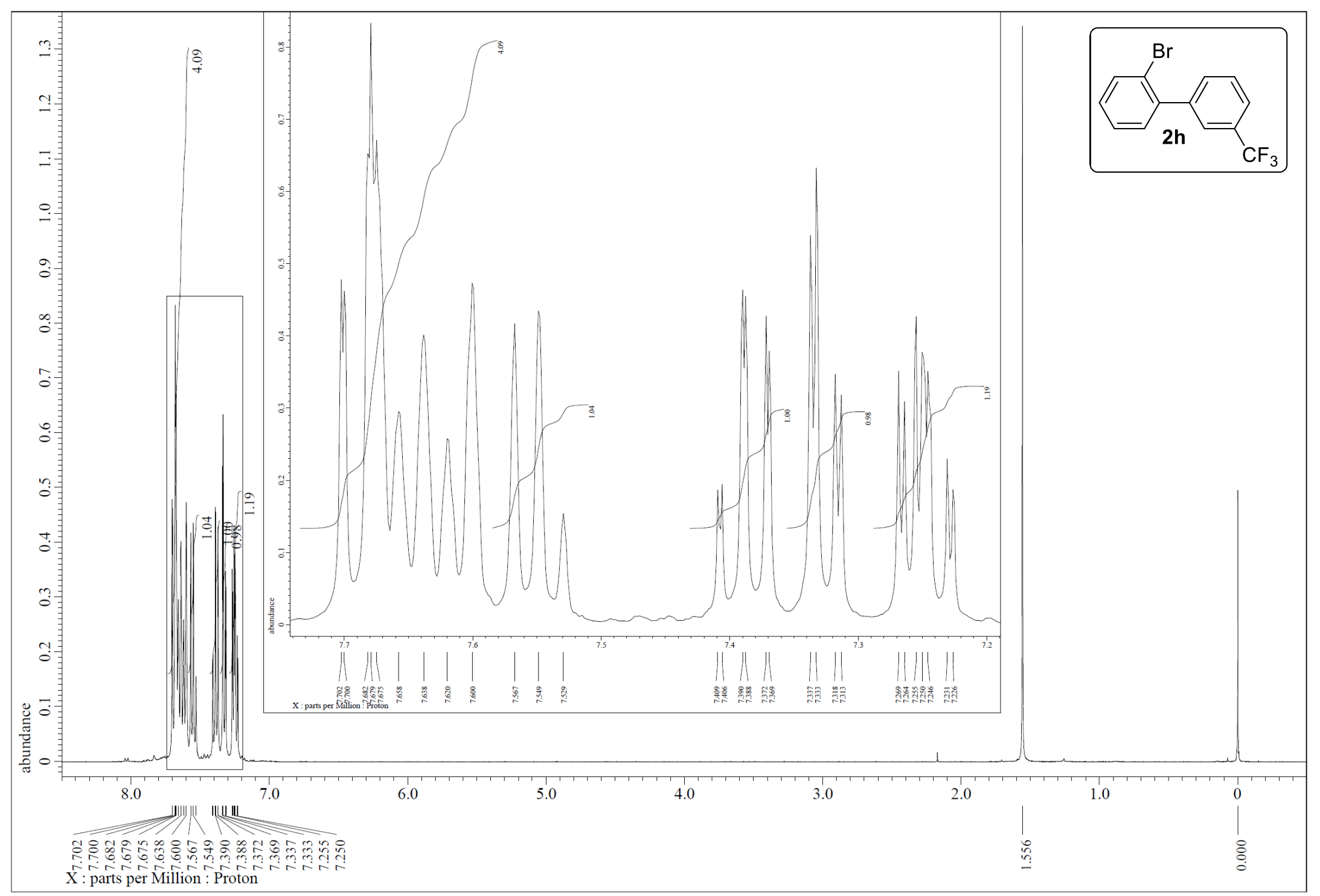

Figure S27. ${ }^{1} \mathrm{H}$ NMR (400 MHz) spectrum of $\mathbf{2 h}\left(\mathrm{CDCl}_{3}, \mathrm{rt}\right)$ 


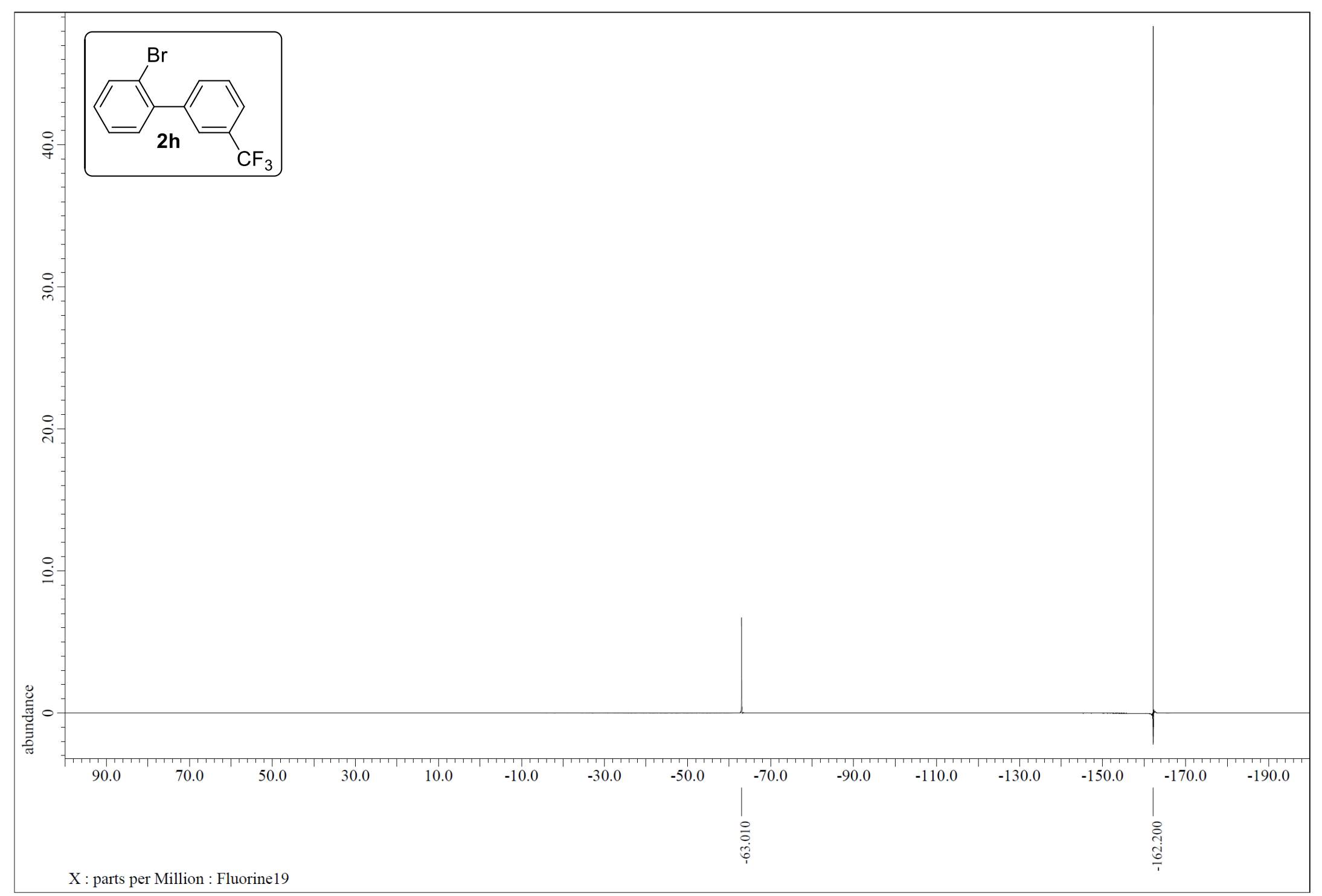

Figure S28. ${ }^{19} \mathrm{~F}\left\{{ }^{1} \mathrm{H}\right\}$ NMR (376 MHz) spectrum of $\mathbf{2 h}\left(\mathrm{CDCl}_{3}, \mathrm{rt}\right)$ 


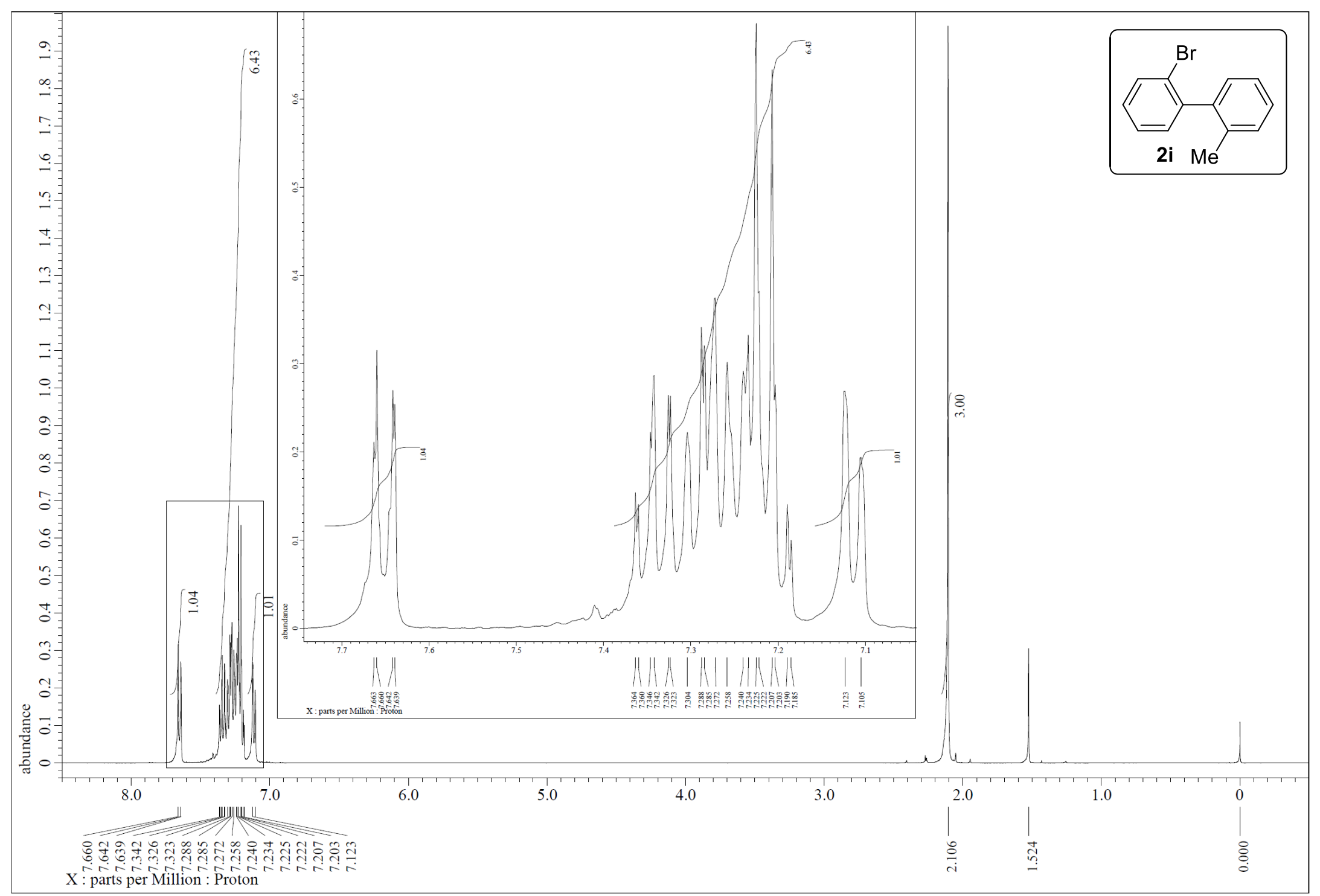

Figure S29. ${ }^{1} \mathrm{H}$ NMR $(400 \mathrm{MHz})$ spectrum of $\mathbf{2 i}\left(\mathrm{CDCl}_{3}, \mathrm{rt}\right)$ 


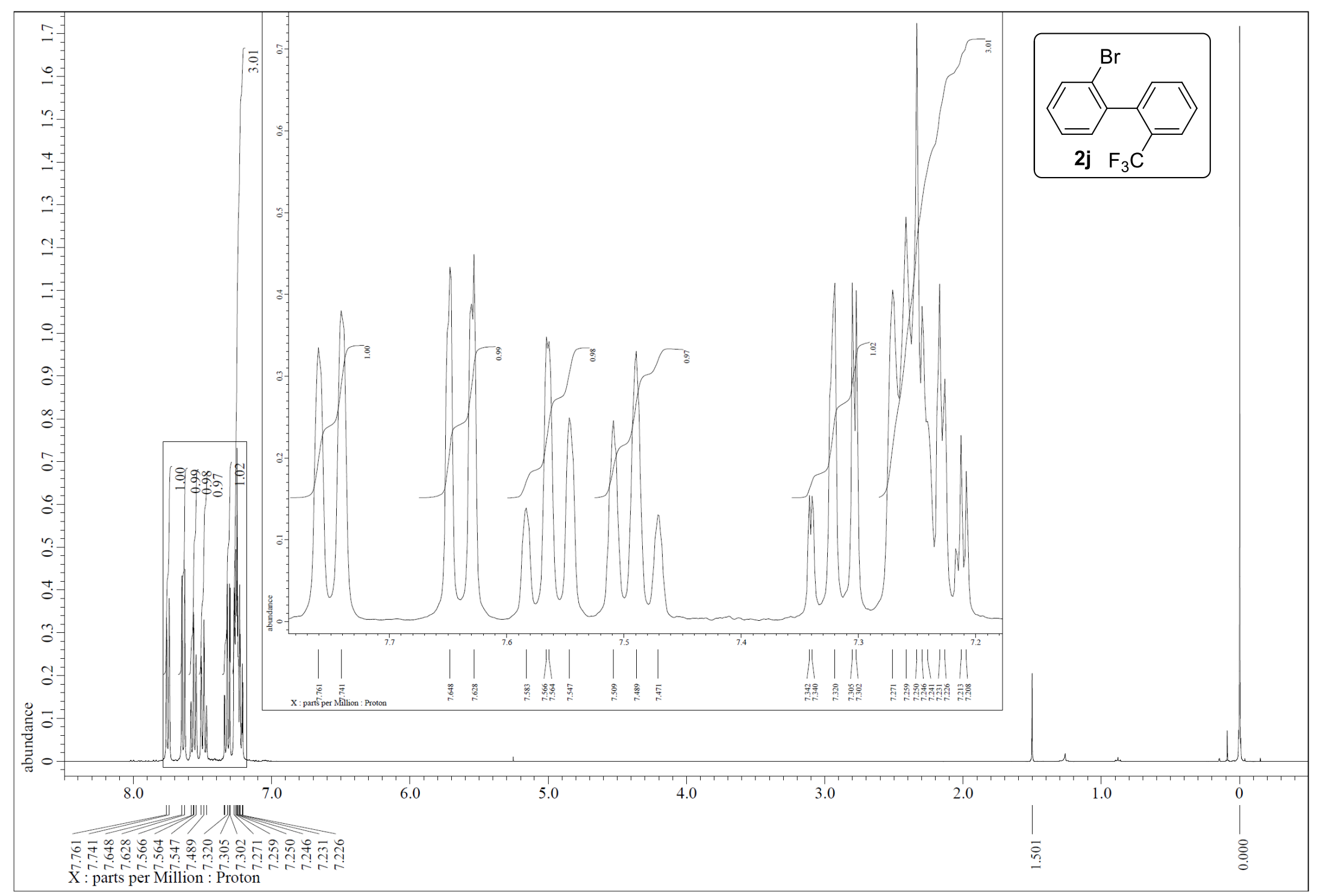

Figure S30. ${ }^{1} \mathrm{H}$ NMR (400 MHz) spectrum of $\mathbf{2 j}\left(\mathrm{CDCl}_{3}, \mathrm{rt}\right)$ 


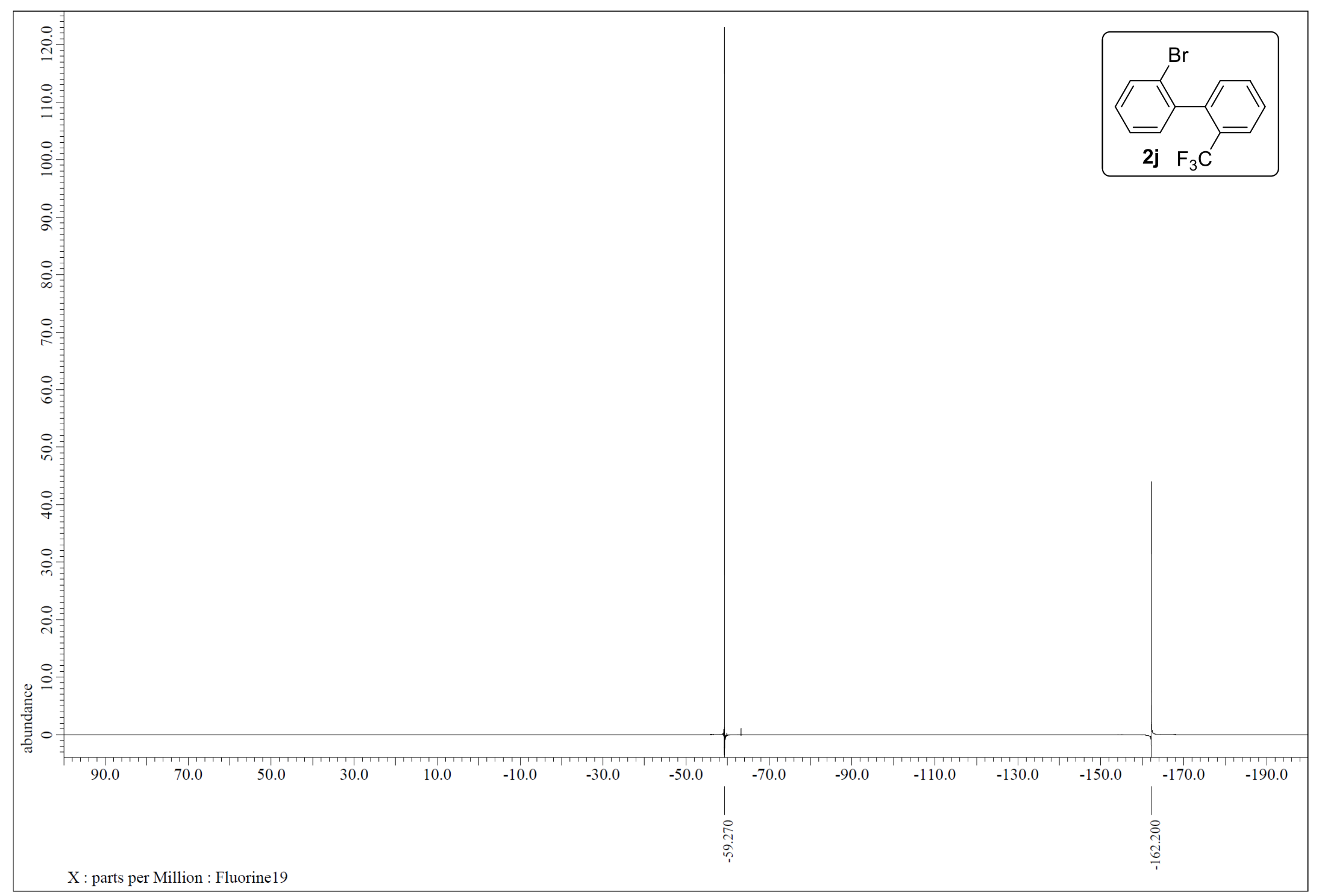

Figure S31. ${ }^{19} \mathrm{~F}\left\{{ }^{1} \mathrm{H}\right\} \mathrm{NMR}(376 \mathrm{MHz})$ spectrum of $\mathbf{2} \mathbf{j}\left(\mathrm{CDCl}_{3}, \mathrm{rt}\right)$ 


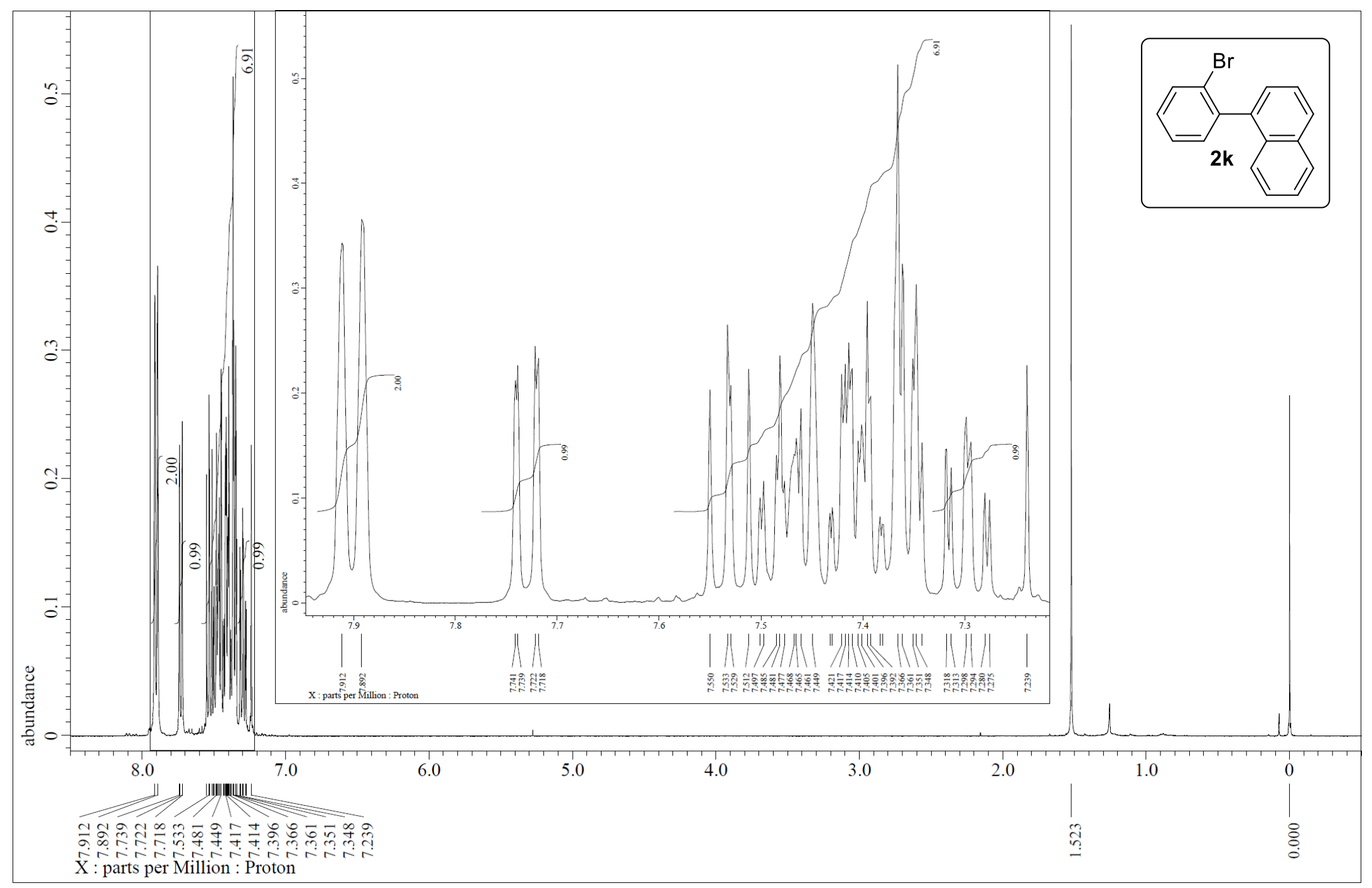

Figure S32. ${ }^{1} \mathrm{H}$ NMR (400 MHz) spectrum of $\mathbf{2 k}\left(\mathrm{CDCl}_{3}, \mathrm{rt}\right)$ 


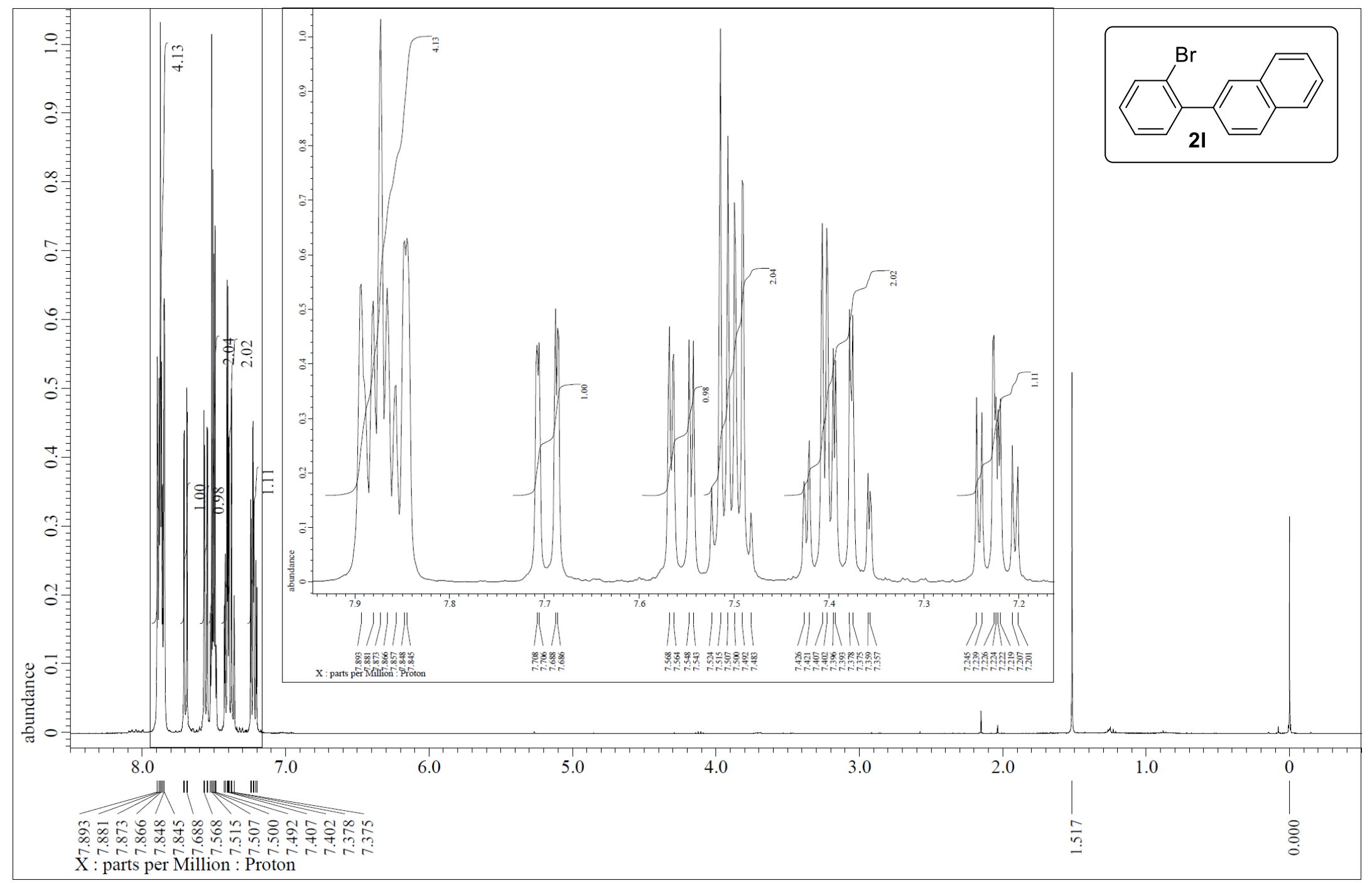

Figure S33. ${ }^{1} \mathrm{H}$ NMR $(400 \mathrm{MHz})$ spectrum of $2 \mathbf{l}\left(\mathrm{CDCl}_{3}, \mathrm{rt}\right)$ 


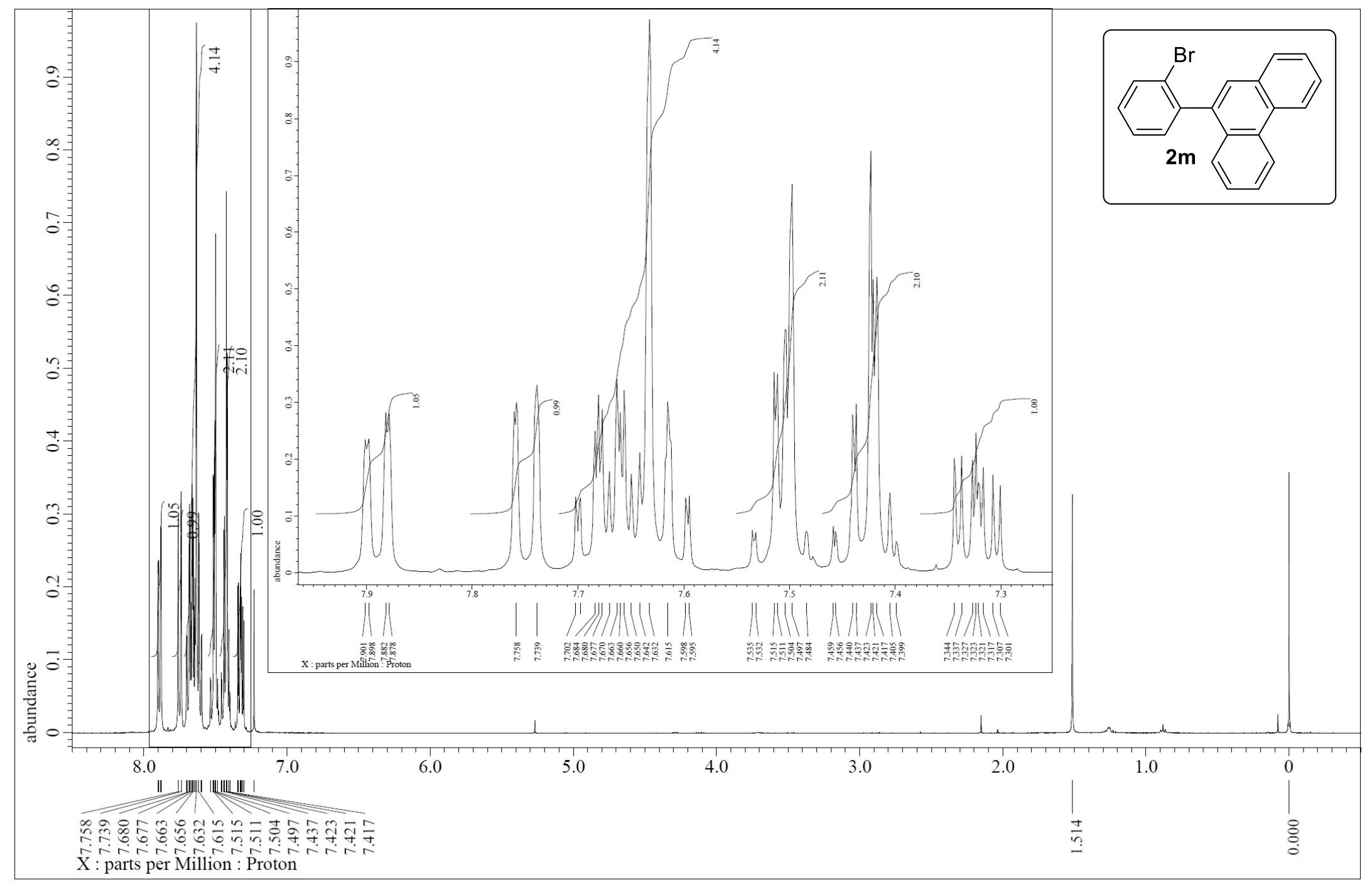

Figure S34. ${ }^{1} \mathrm{H}$ NMR $(400 \mathrm{MHz})$ spectrum of $\mathbf{2 m}\left(\mathrm{CDCl}_{3}, \mathrm{rt}\right)$ 


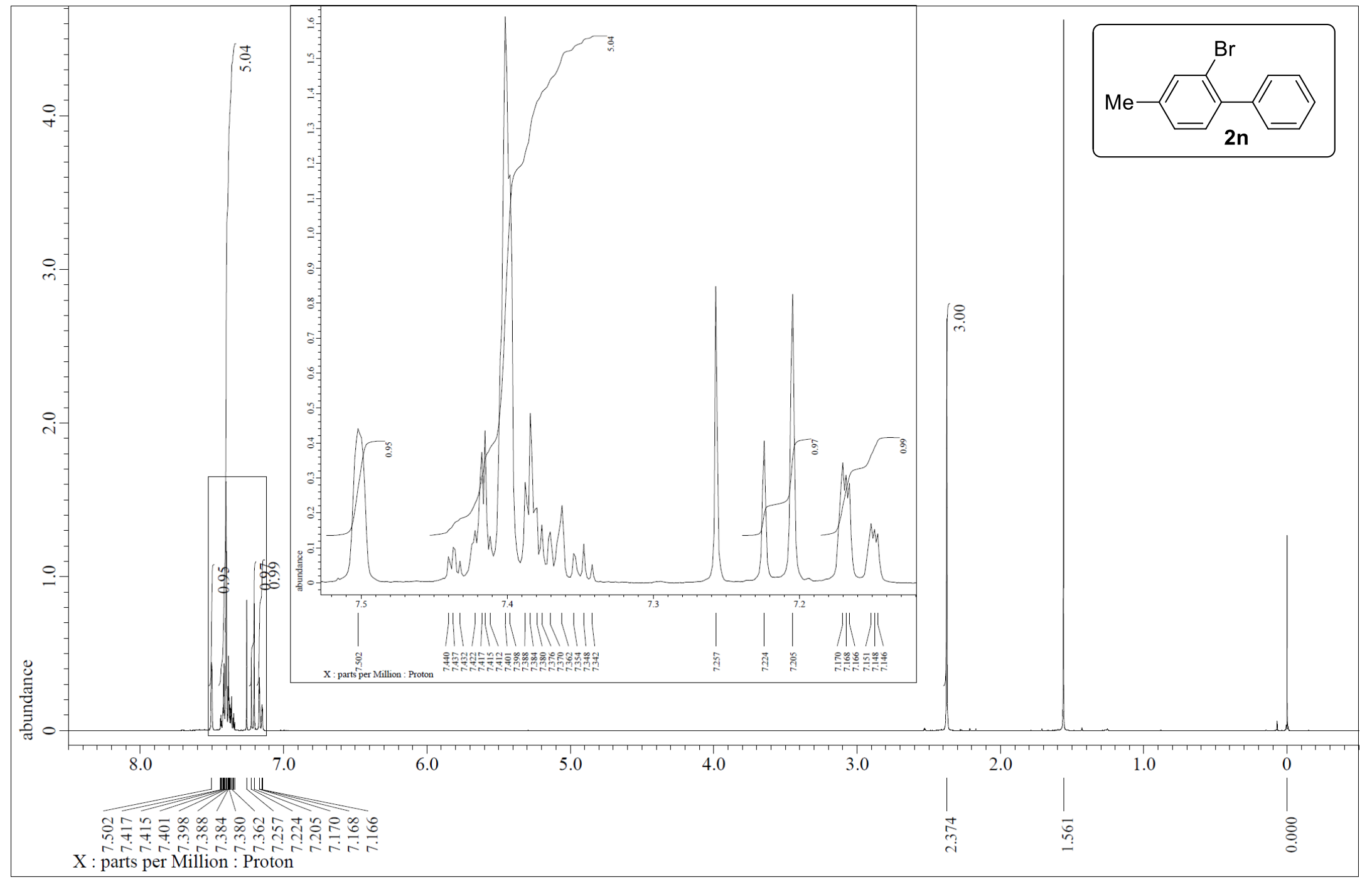

Figure S35. ${ }^{1} \mathrm{H}$ NMR $(400 \mathrm{MHz})$ spectrum of $\mathbf{2 n}\left(\mathrm{CDCl}_{3}, \mathrm{rt}\right)$ 


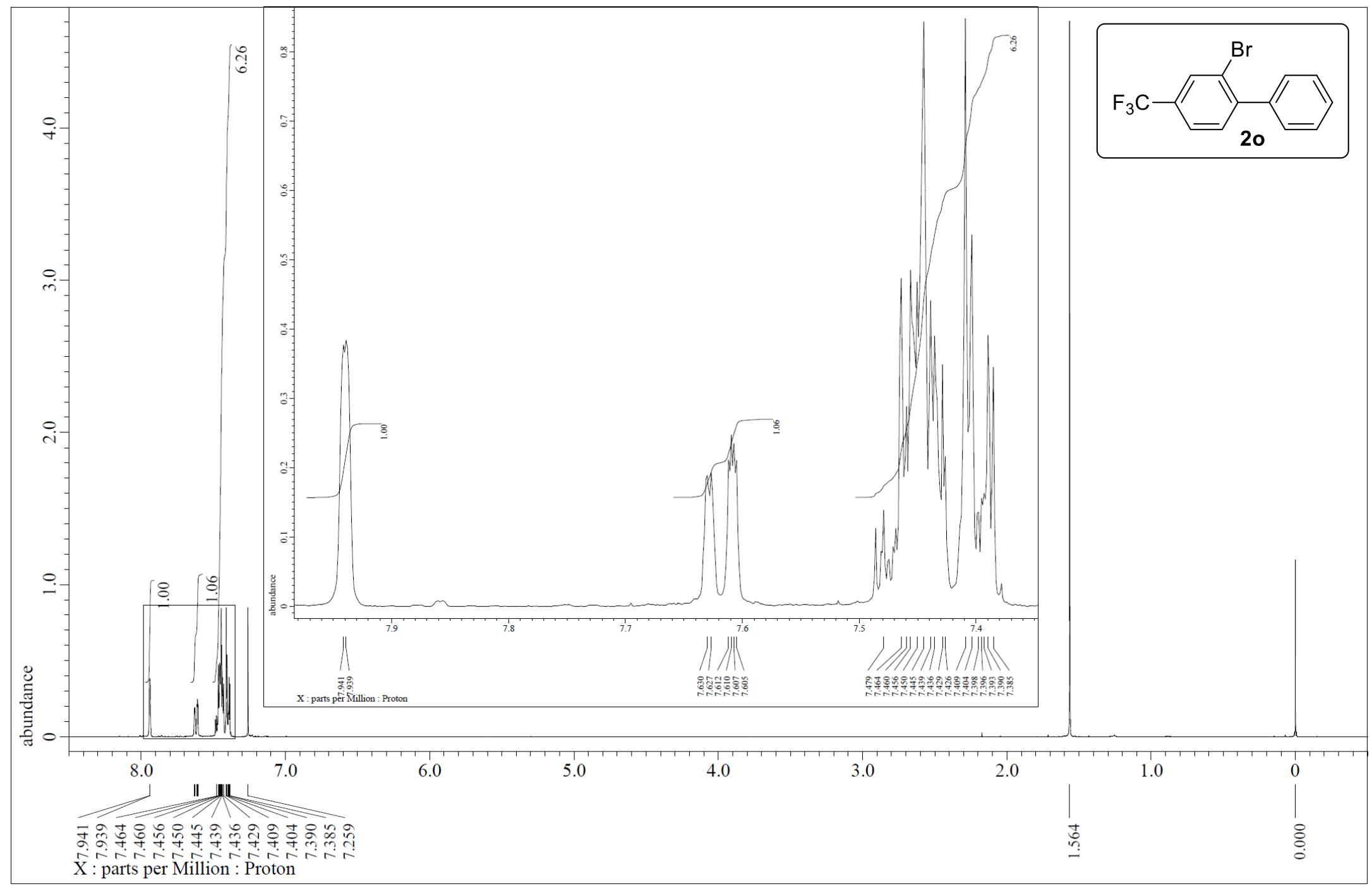

Figure S36. ${ }^{1} \mathrm{H}$ NMR $(400 \mathrm{MHz})$ spectrum of $2 \mathbf{o}\left(\mathrm{CDCl}_{3}, \mathrm{rt}\right)$ 


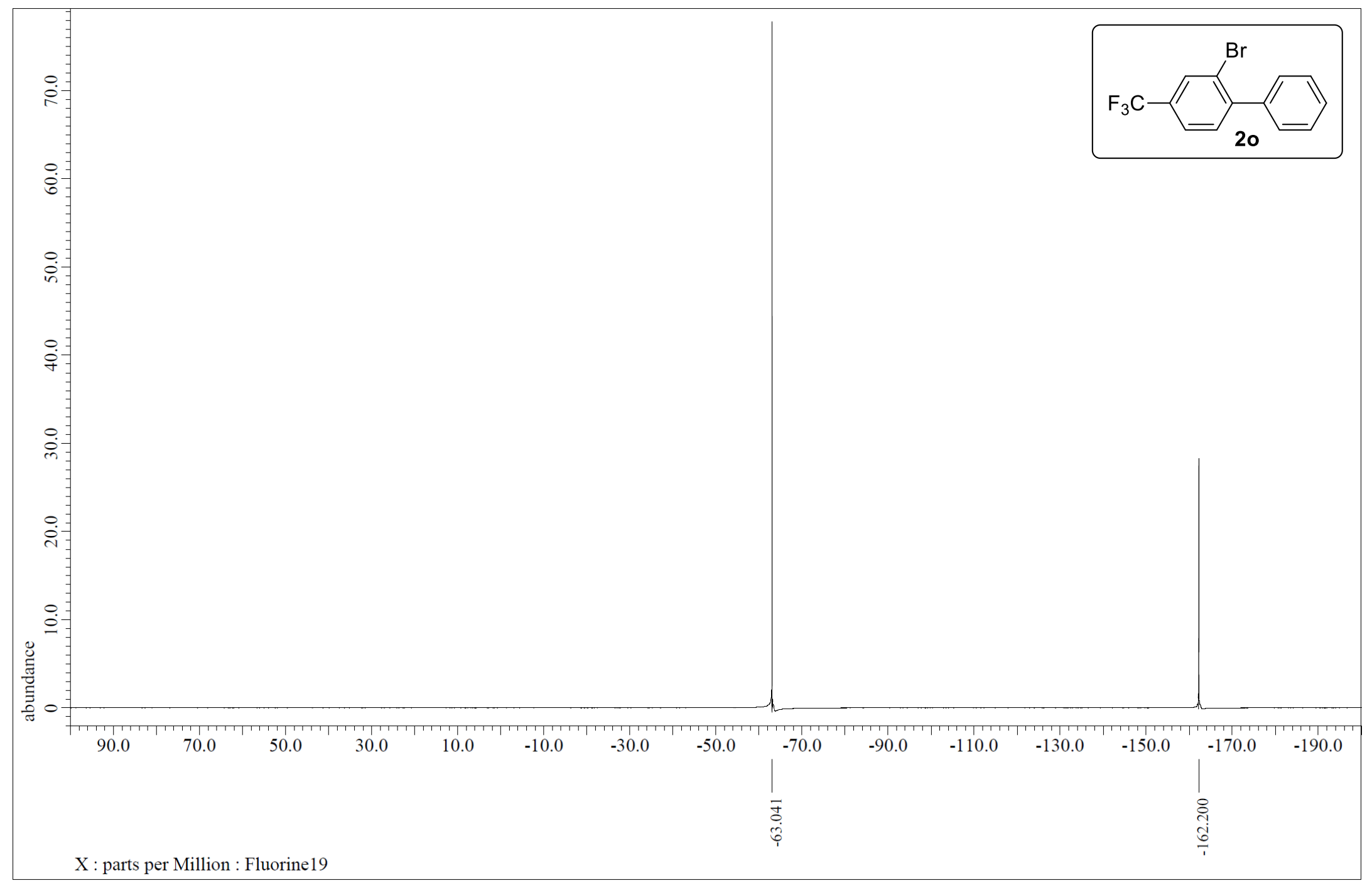

Figure S37. ${ }^{19} \mathrm{~F}\left\{{ }^{1} \mathrm{H}\right\}$ NMR $(376 \mathrm{MHz})$ spectrum of $20\left(\mathrm{CDCl}_{3}, \mathrm{rt}\right)$ 


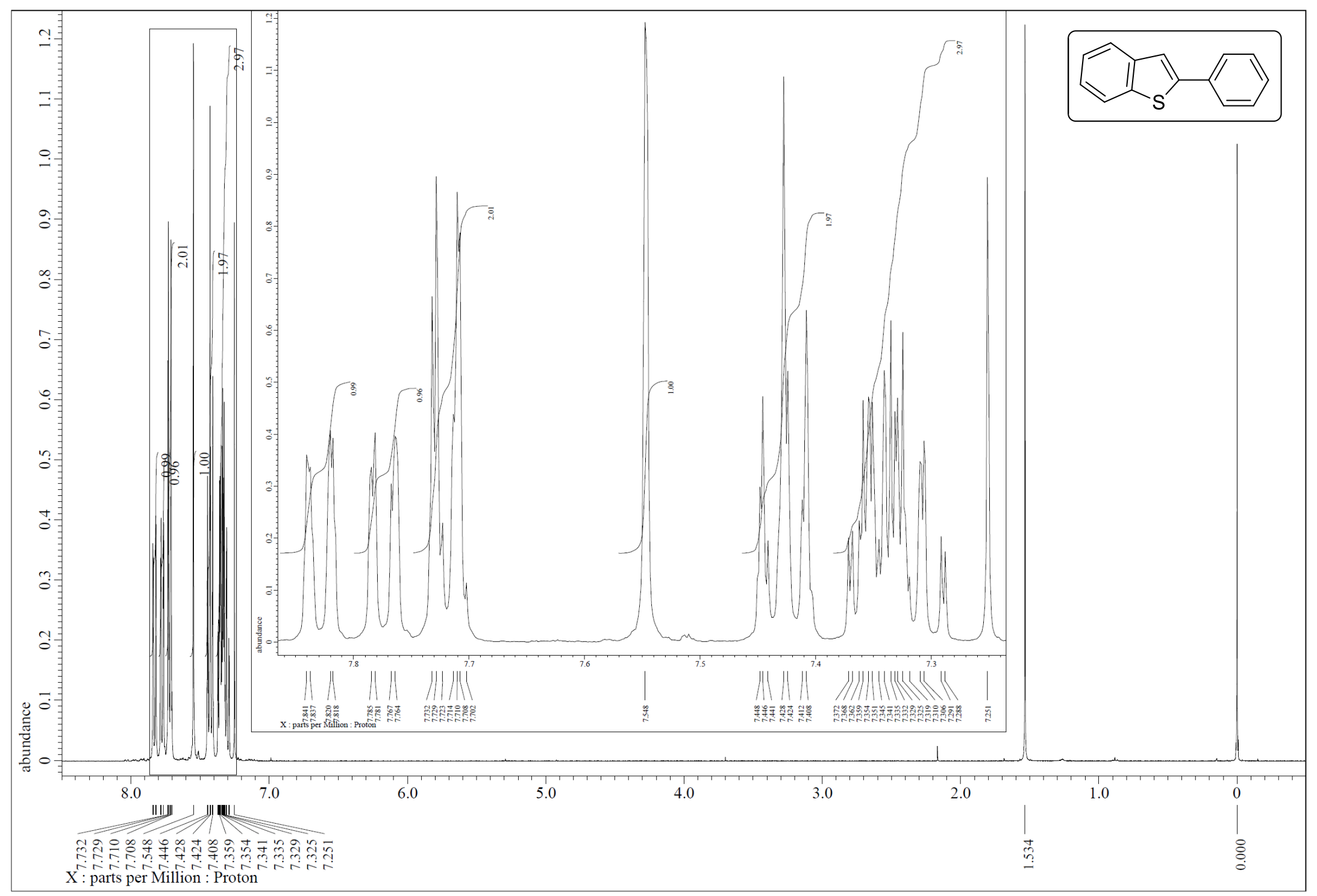

Figure S38. ${ }^{1} \mathrm{H}$ NMR $(400 \mathrm{MHz})$ spectrum of 2-phenylbenzo[b]thiophene $\left(\mathrm{CDCl}_{3}, \mathrm{rt}\right)$ 


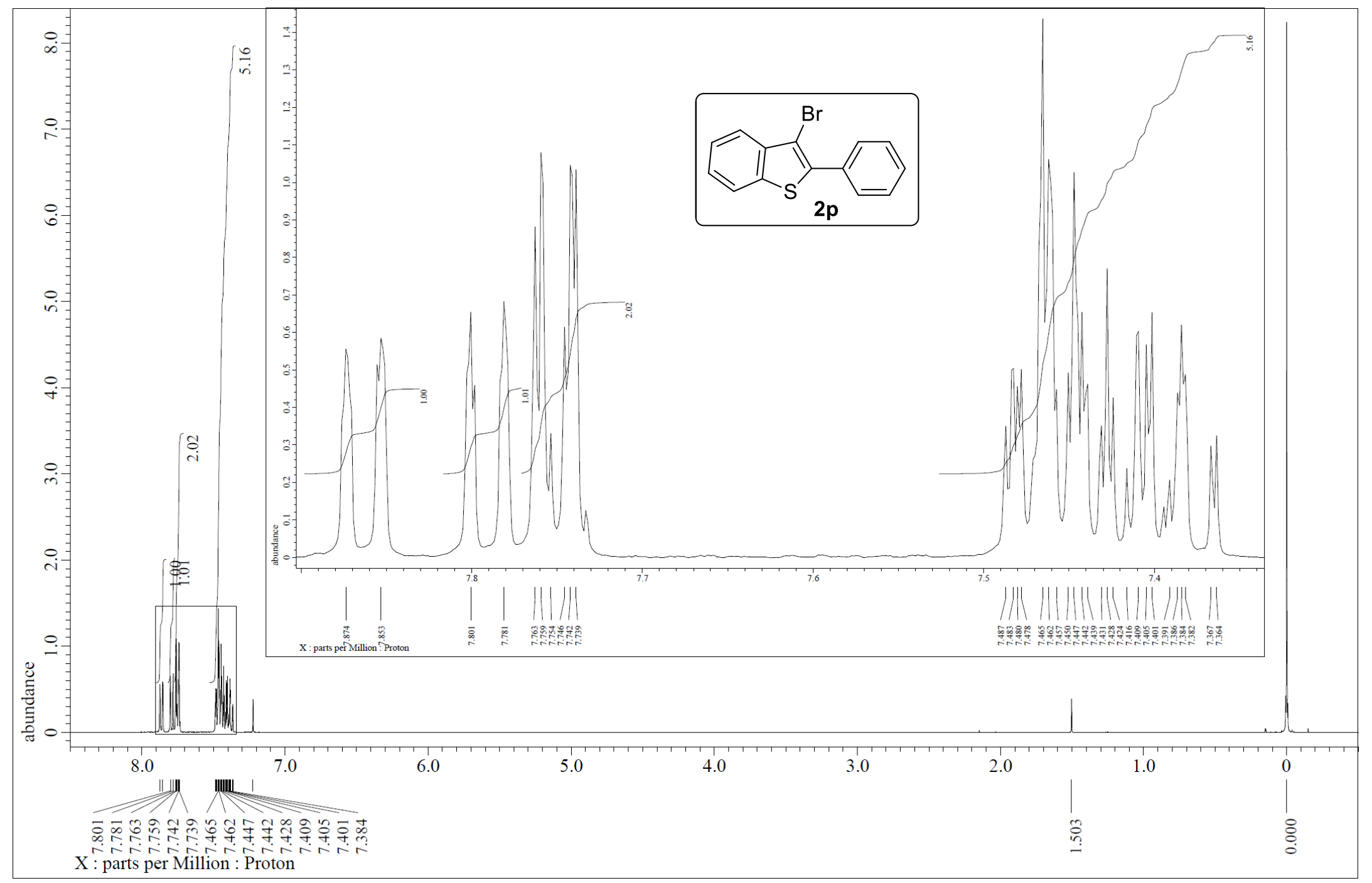

Figure S39. ${ }^{1} \mathrm{H}$ NMR (400 MHz) spectrum of $\mathbf{2 p}\left(\mathrm{CDCl}_{3}, \mathrm{rt}\right)$ 


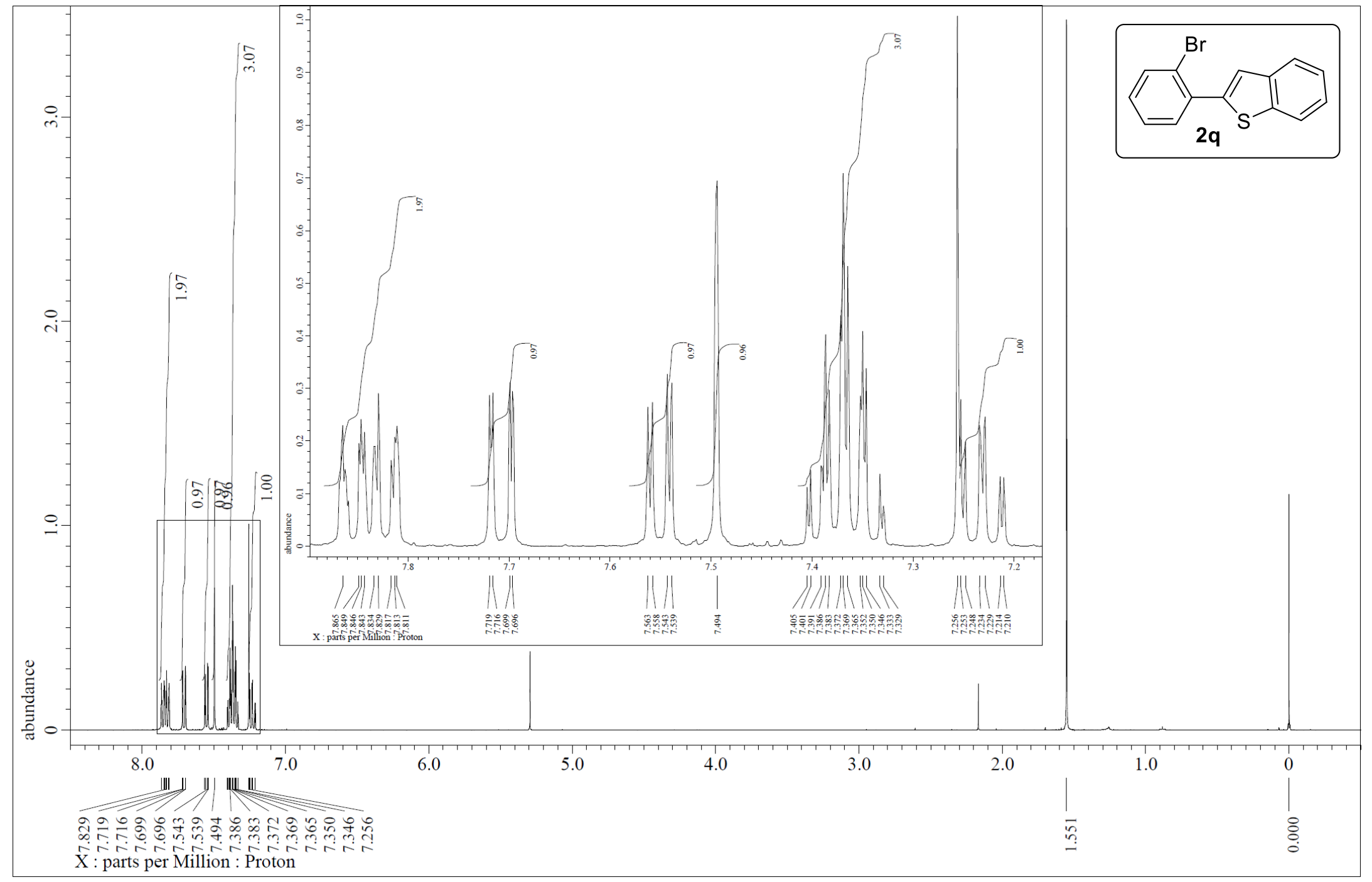

Figure S40. ${ }^{1} \mathrm{H}$ NMR $(400 \mathrm{MHz})$ spectrum of $\mathbf{2 q}\left(\mathrm{CDCl}_{3}, \mathrm{rt}\right)$ 


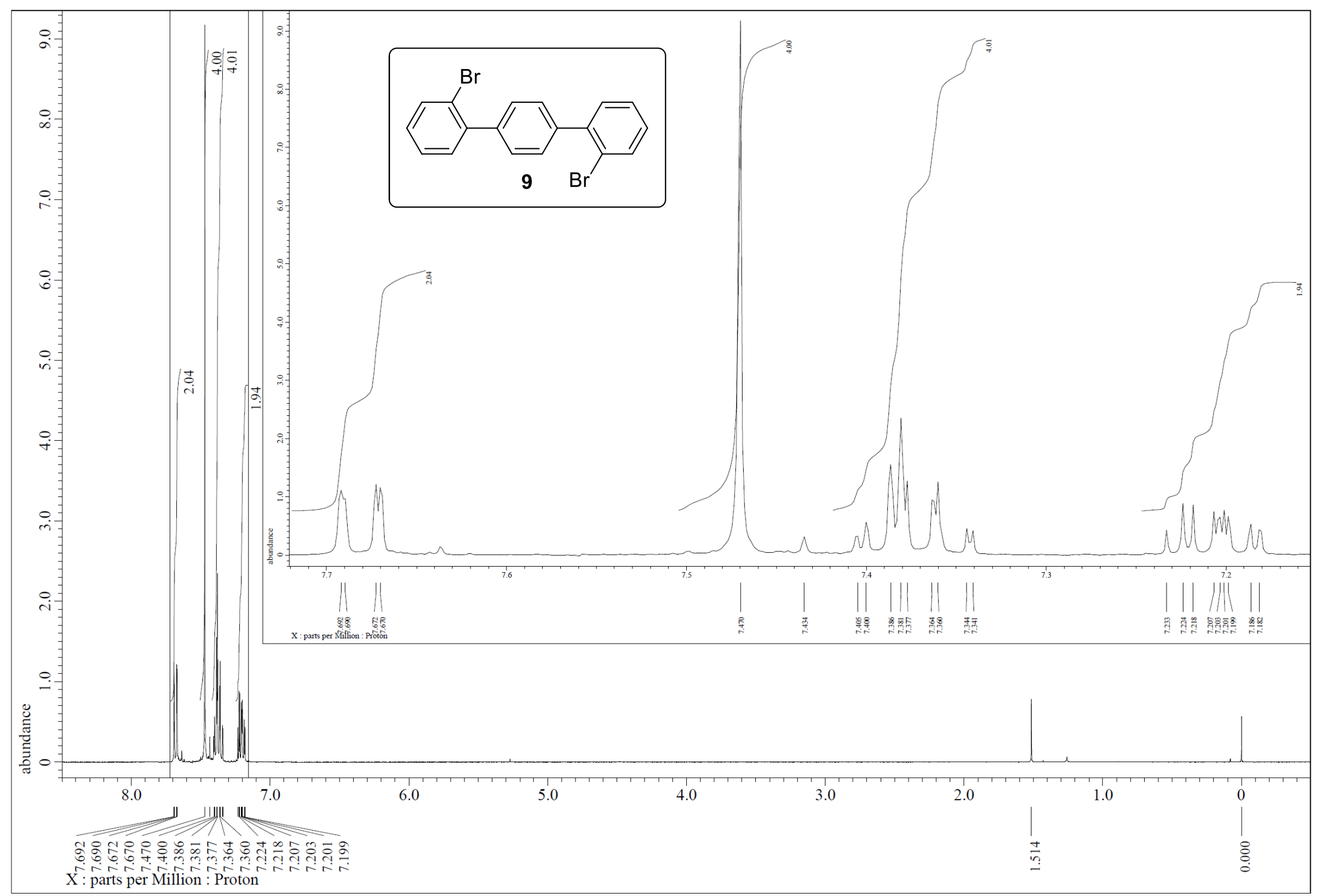

Figure S41. ${ }^{1} \mathrm{H}$ NMR $(400 \mathrm{MHz})$ spectrum of $\mathbf{9}\left(\mathrm{CDCl}_{3}, \mathrm{rt}\right)$ 


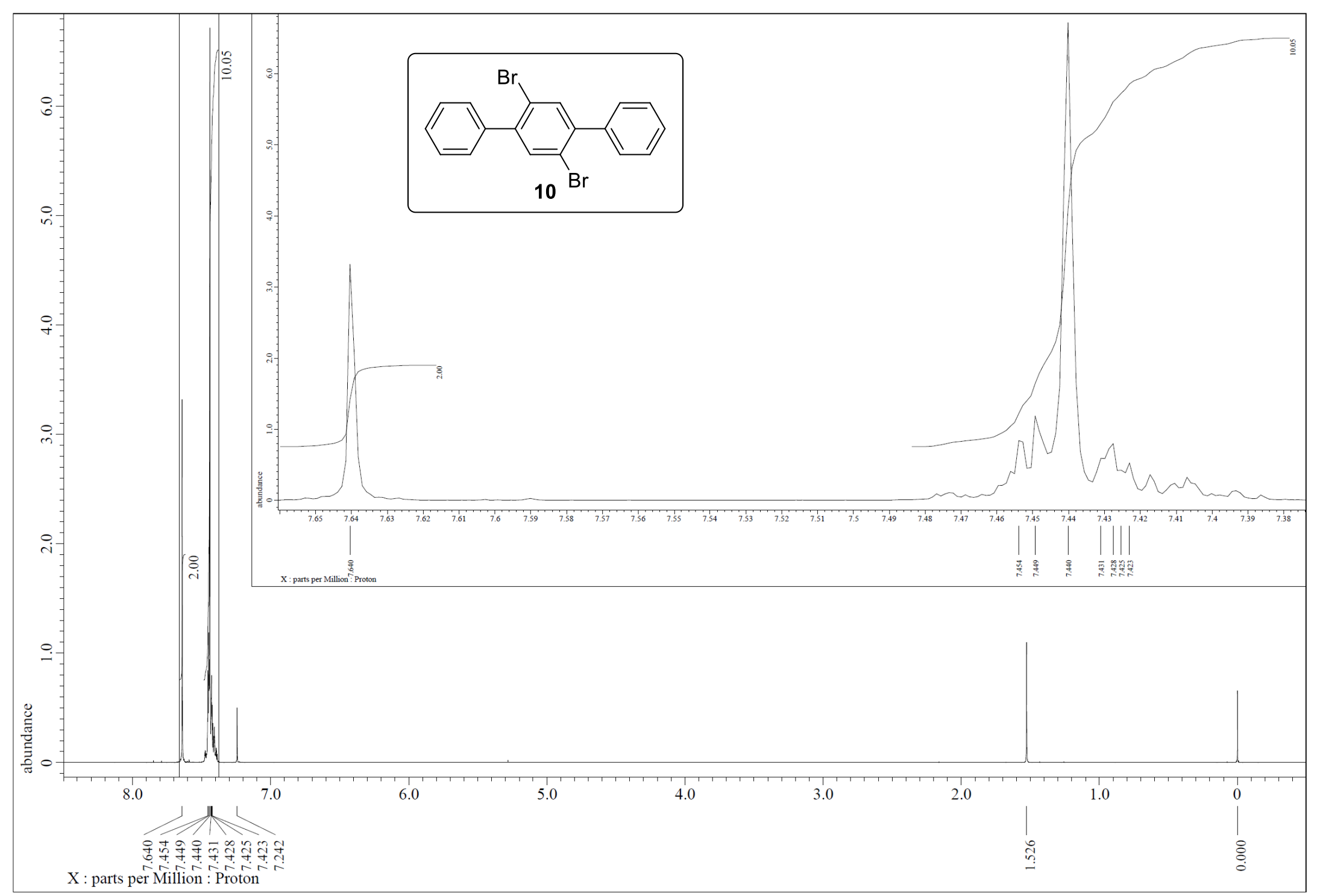

Figure S42. ${ }^{1} \mathrm{H}$ NMR $(400 \mathrm{MHz})$ spectrum of $\mathbf{1 0}\left(\mathrm{CDCl}_{3}, \mathrm{rt}\right)$ 


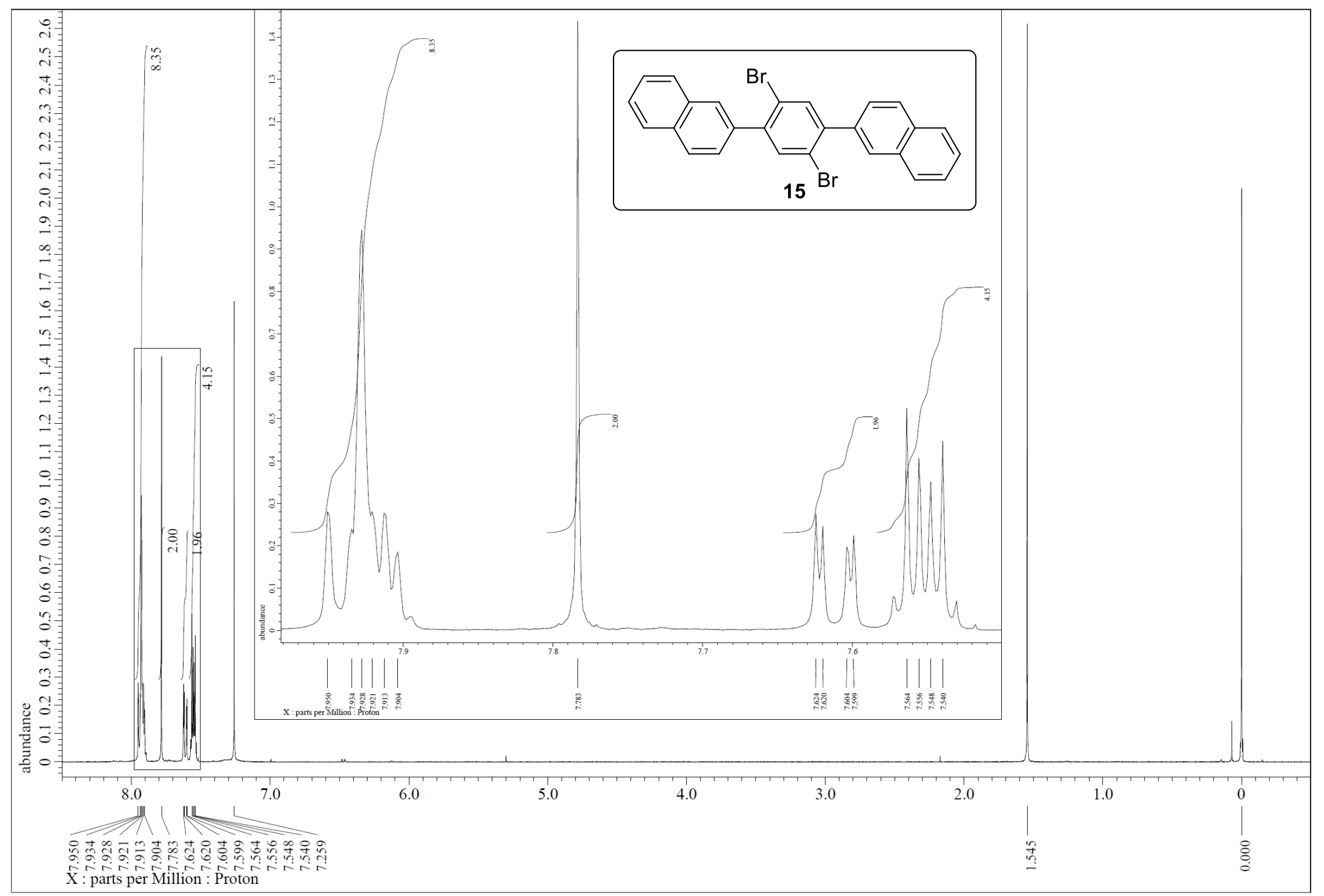

Figure S43. ${ }^{1} \mathrm{H}$ NMR $(400 \mathrm{MHz})$ spectrum of $15\left(\mathrm{CDCl}_{3}, \mathrm{rt}\right)$ 


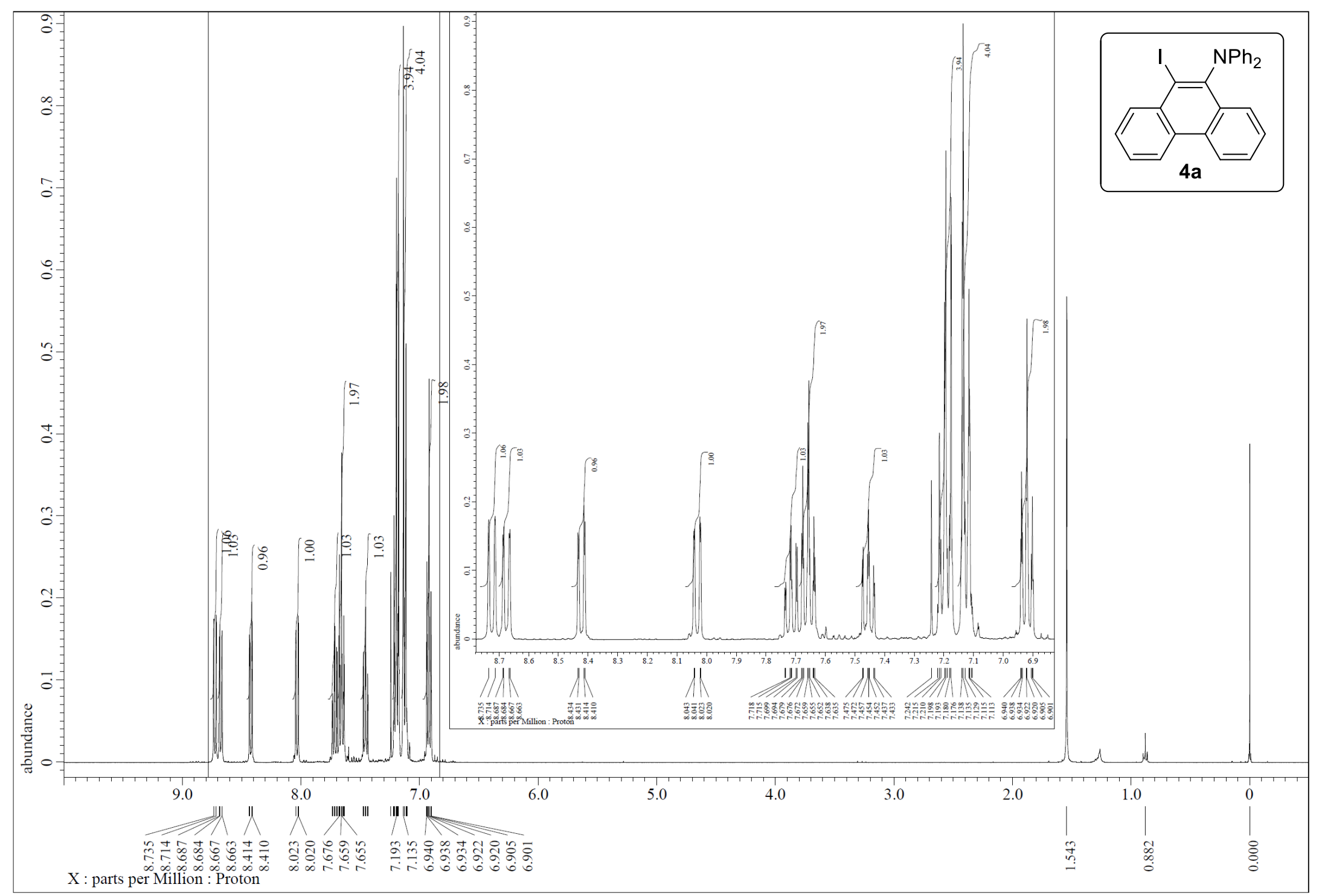

Figure S44. ${ }^{1} \mathrm{H}$ NMR (400 MHz) spectrum of $\mathbf{4 a}\left(\mathrm{CDCl}_{3}, \mathrm{rt}\right)$ 


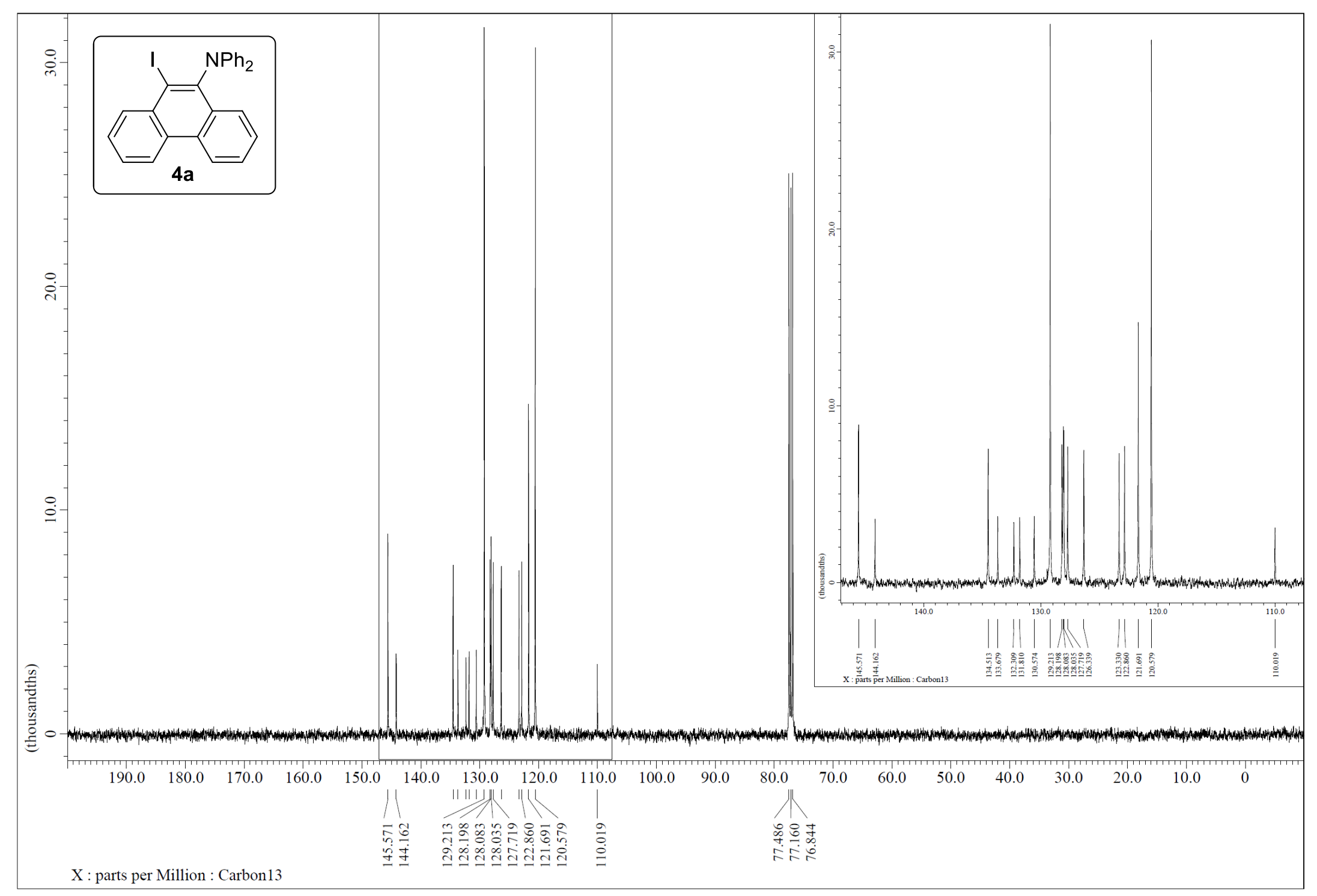

Figure S45. ${ }^{13} \mathrm{C}\left\{{ }^{1} \mathrm{H}\right\}$ NMR $(101 \mathrm{MHz})$ spectrum of $\mathbf{4 a}\left(\mathrm{CDCl}_{3}, \mathrm{rt}\right)$ 


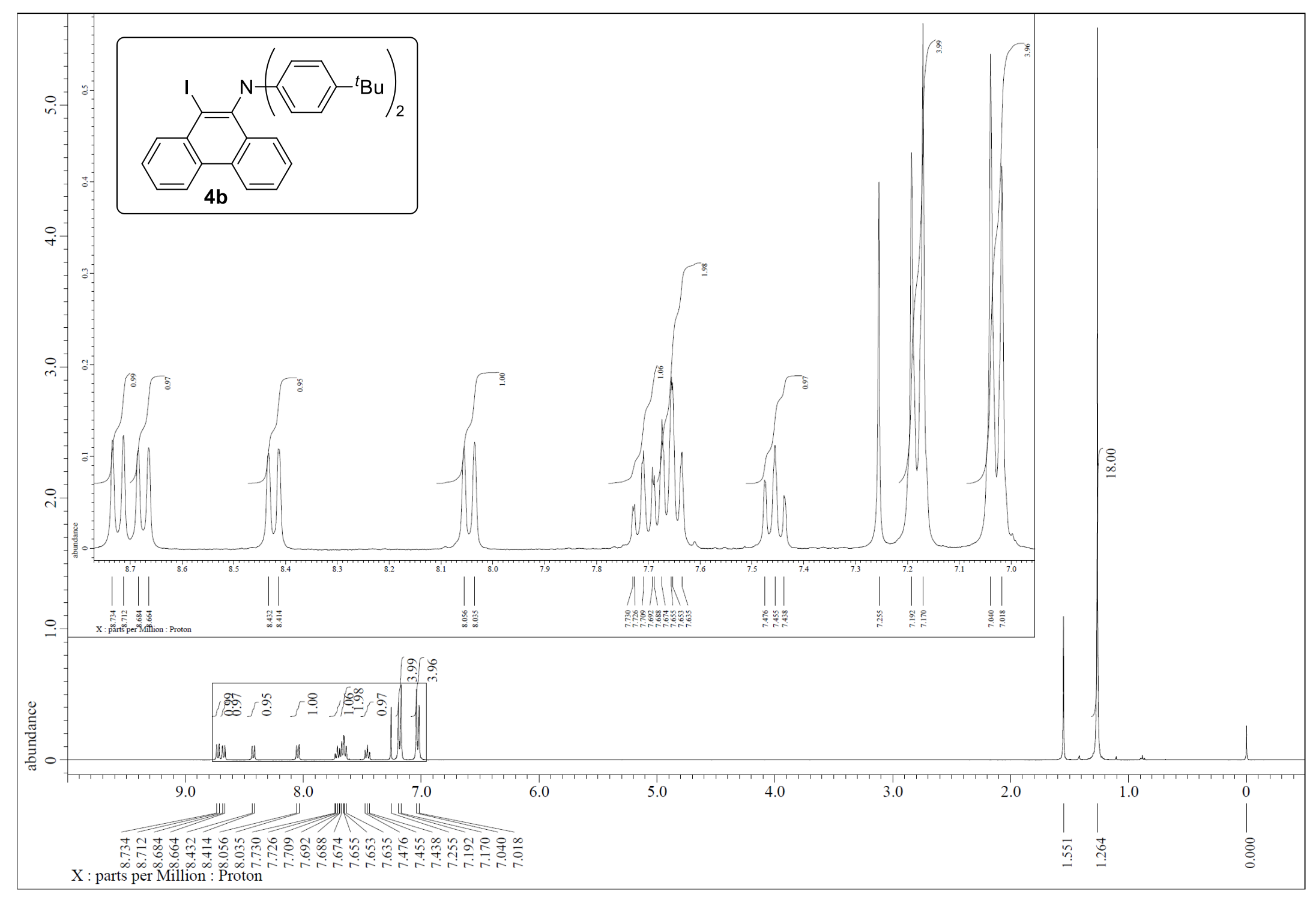

Figure S46. ${ }^{1} \mathrm{H}$ NMR $(400 \mathrm{MHz})$ spectrum of $\mathbf{4 b}\left(\mathrm{CDCl}_{3}, \mathrm{rt}\right)$ 


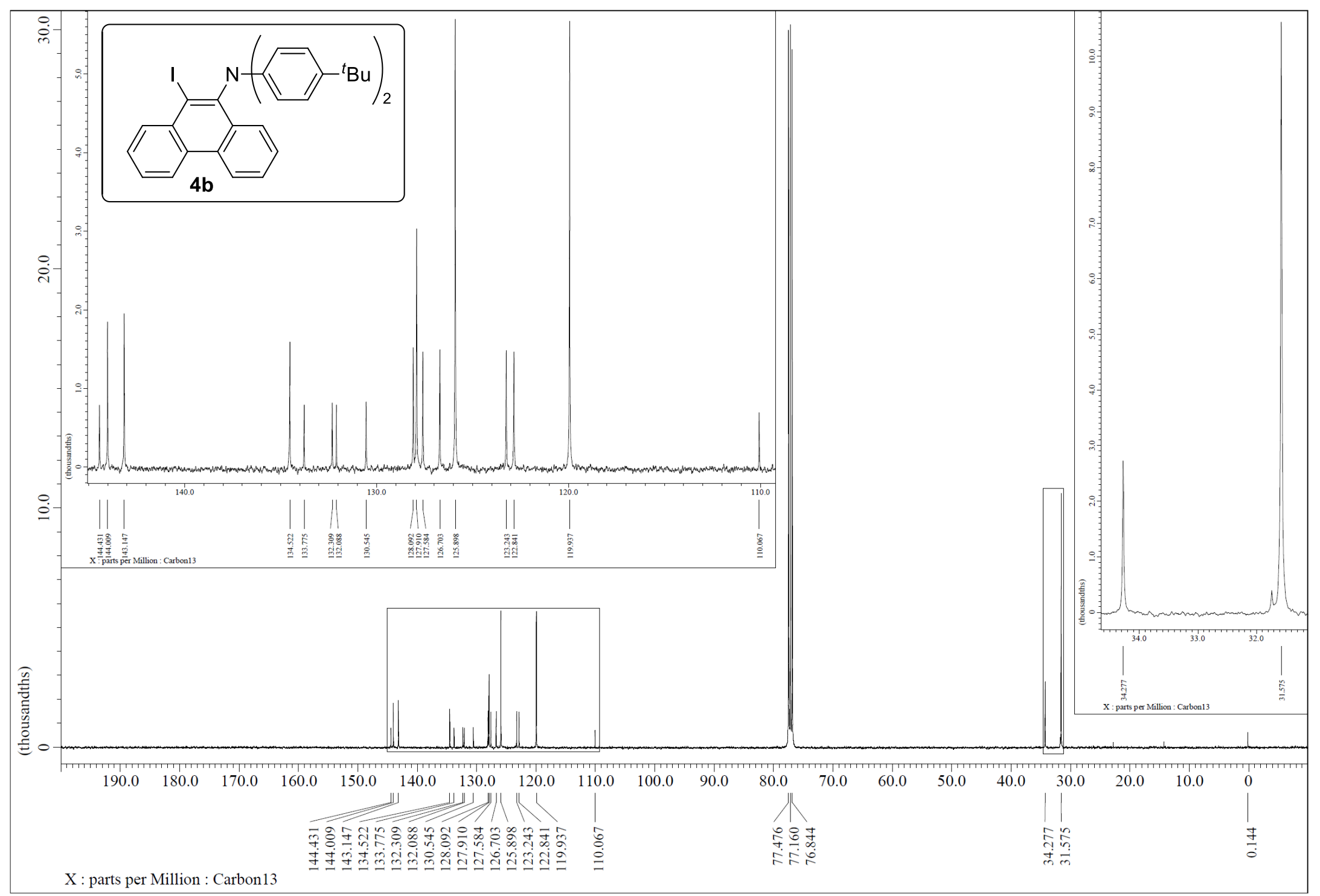

Figure S47. ${ }^{13} \mathrm{C}\left\{{ }^{1} \mathrm{H}\right\}$ NMR $(101 \mathrm{MHz})$ spectrum of $\mathbf{4 b}\left(\mathrm{CDCl}_{3}, \mathrm{rt}\right)$ 


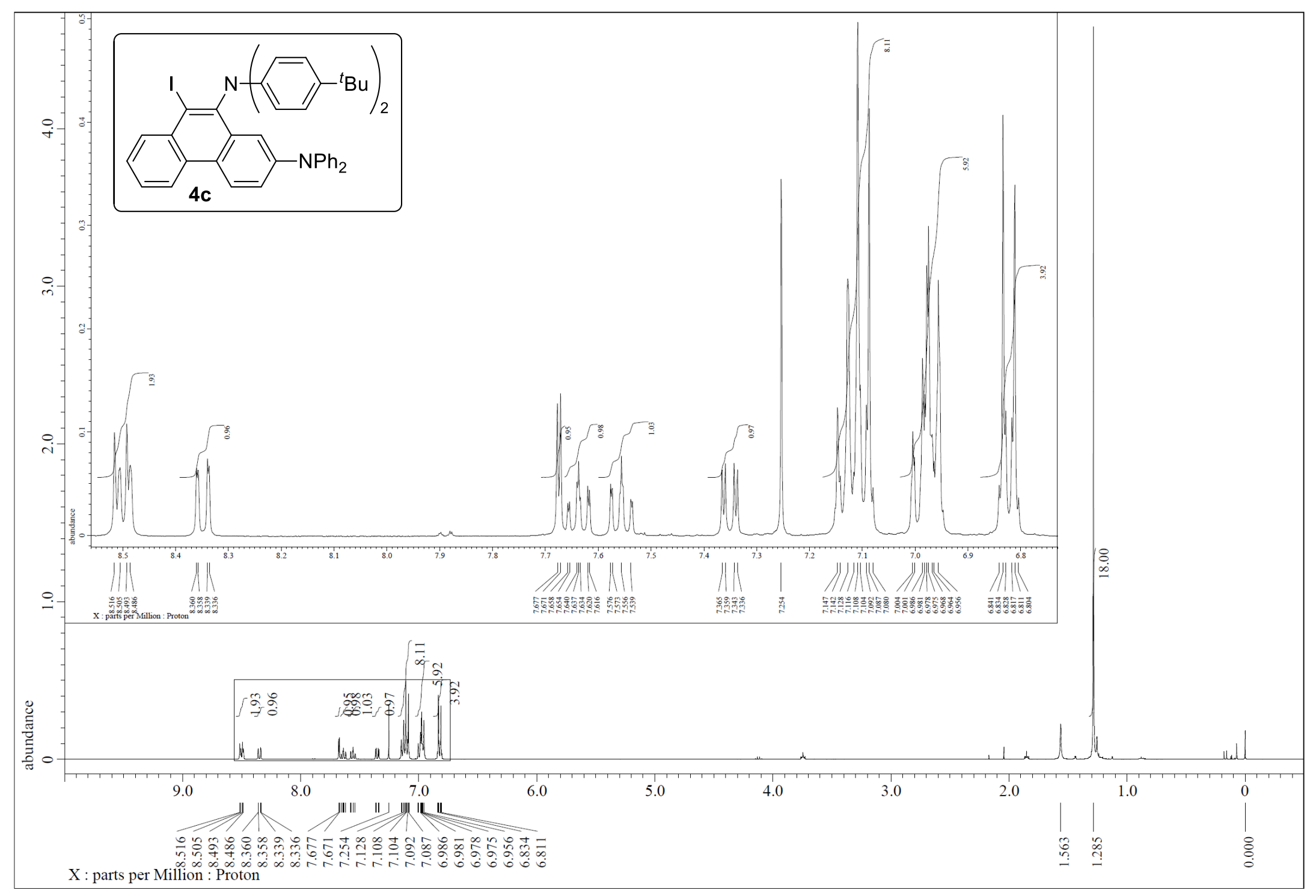

Figure S48. ${ }^{1} \mathrm{H}$ NMR (400 MHz) spectrum of $\mathbf{4 c}\left(\mathrm{CDCl}_{3}, \mathrm{rt}\right)$ 


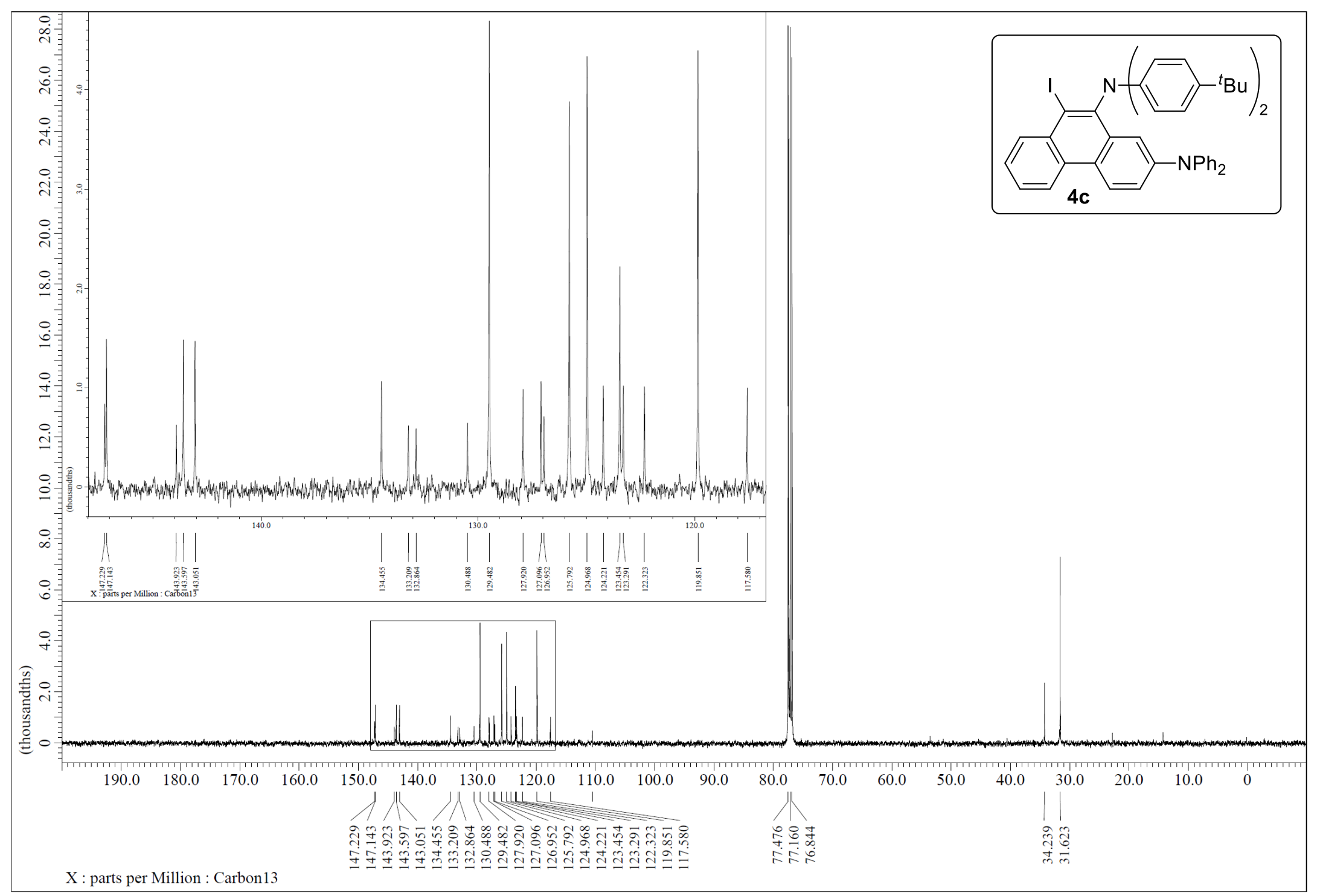

Figure S49. ${ }^{13} \mathrm{C}\left\{{ }^{1} \mathrm{H}\right\} \mathrm{NMR}(101 \mathrm{MHz})$ spectrum of $\mathbf{4 c}\left(\mathrm{CDCl}_{3}, \mathrm{rt}\right)$ 


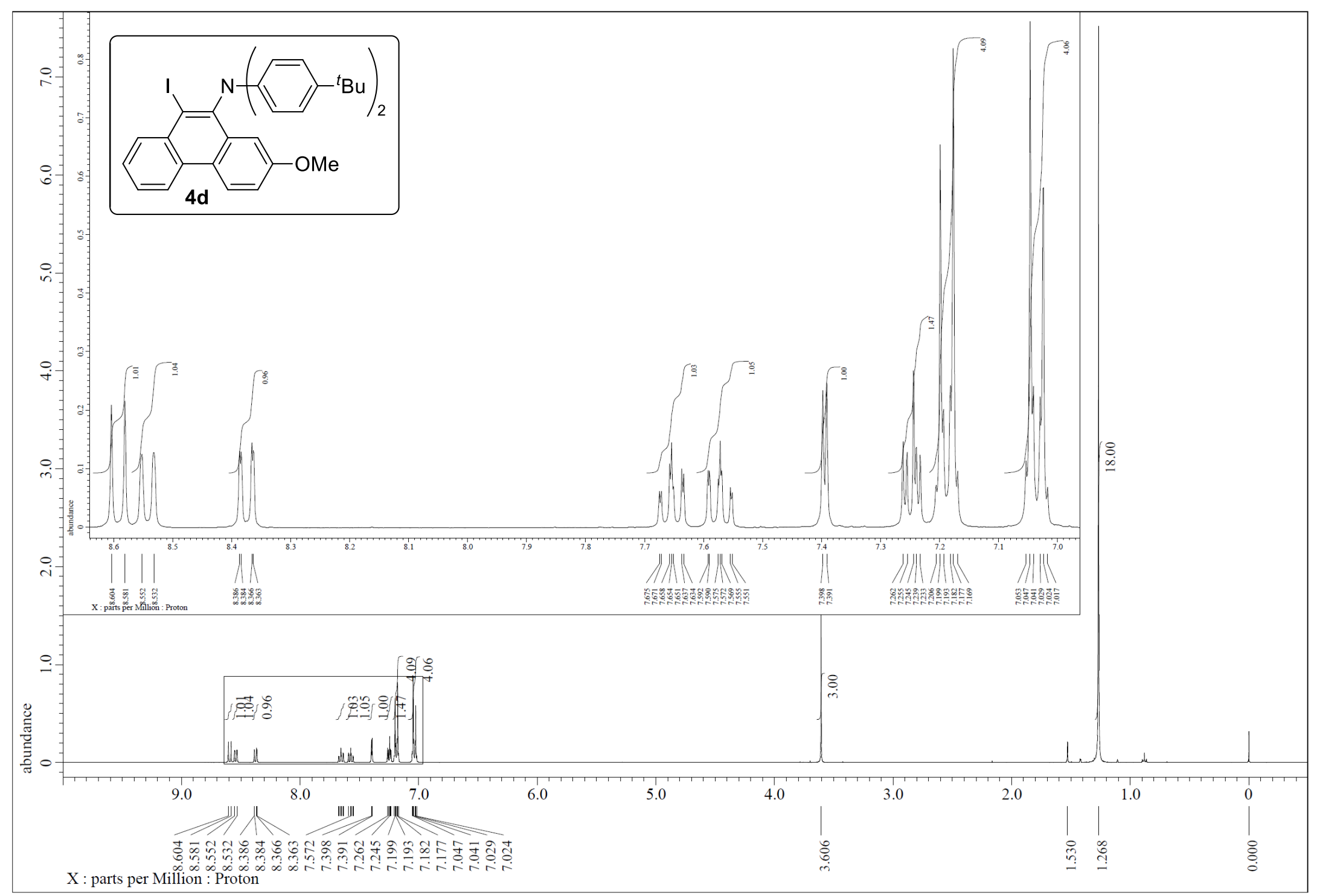

Figure S50. ${ }^{1} \mathrm{H}$ NMR (400 MHz) spectrum of $\mathbf{4 d}\left(\mathrm{CDCl}_{3}, \mathrm{rt}\right)$ 


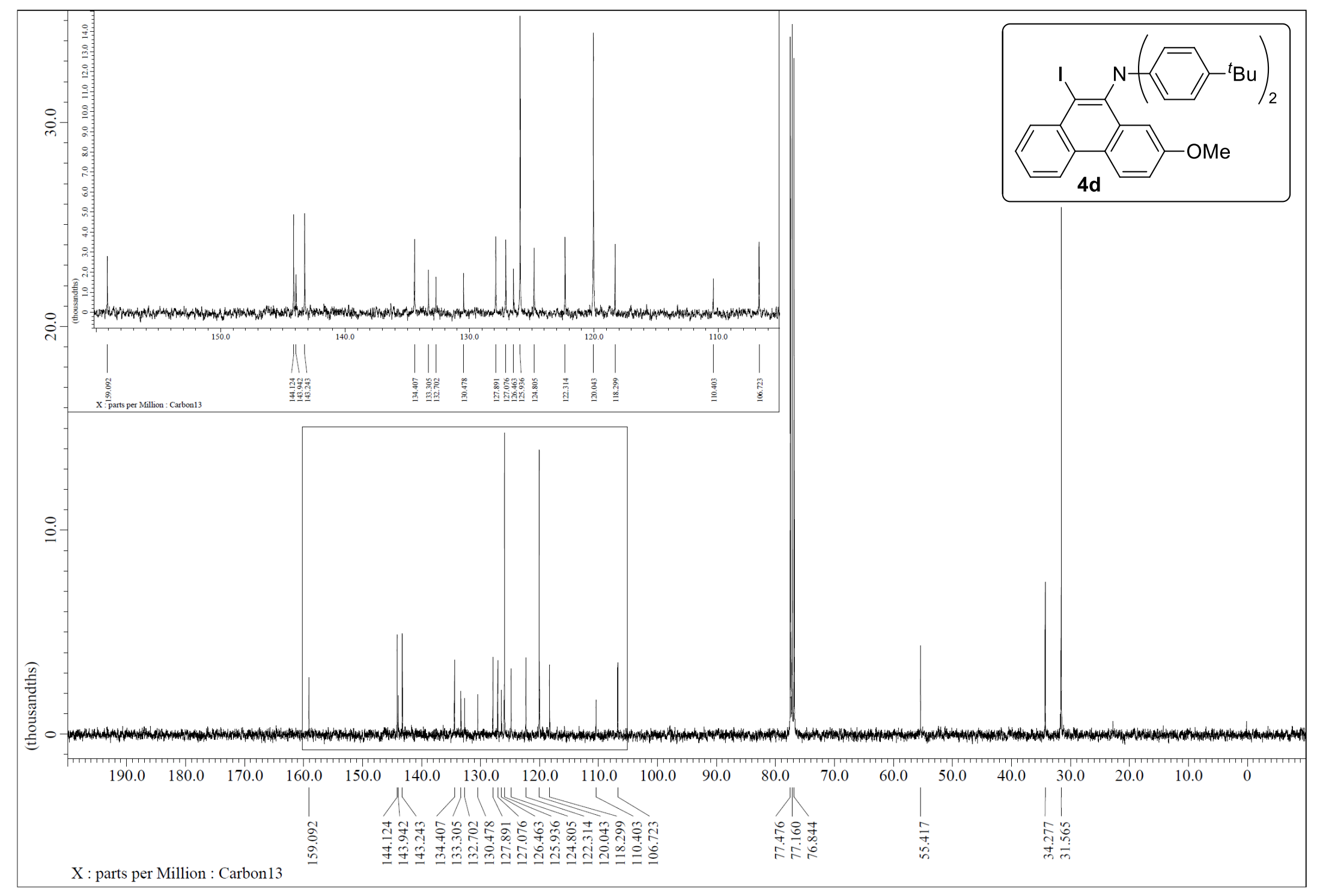

Figure S51. ${ }^{13} \mathrm{C}\left\{{ }^{1} \mathrm{H}\right\}$ NMR (101 MHz) spectrum of $\mathbf{4 d}\left(\mathrm{CDCl}_{3}, \mathrm{rt}\right)$ 


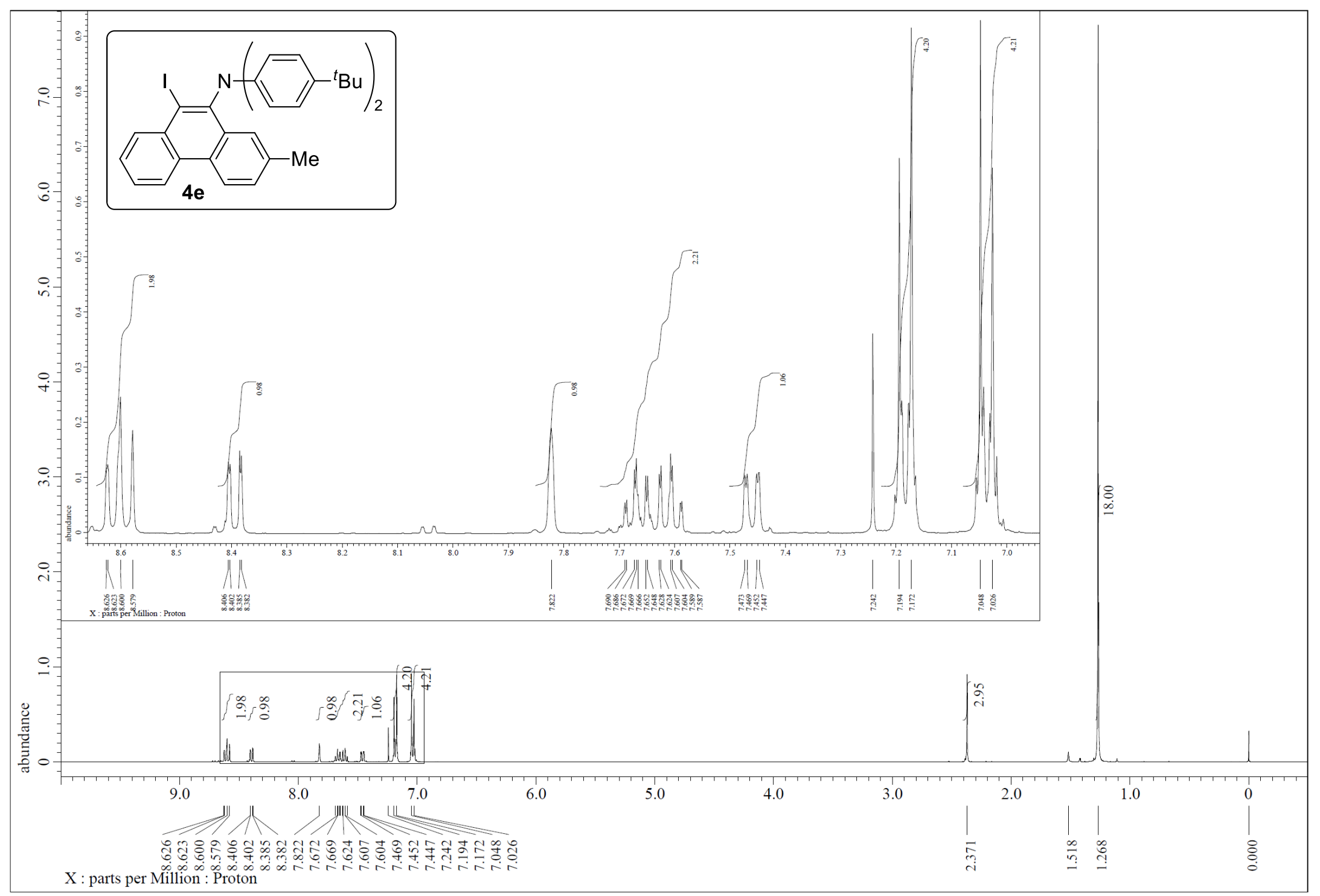

Figure S52. ${ }^{1} \mathrm{H}$ NMR $(400 \mathrm{MHz})$ spectrum of $4 \mathbf{e}\left(\mathrm{CDCl}_{3}, \mathrm{rt}\right)$ 


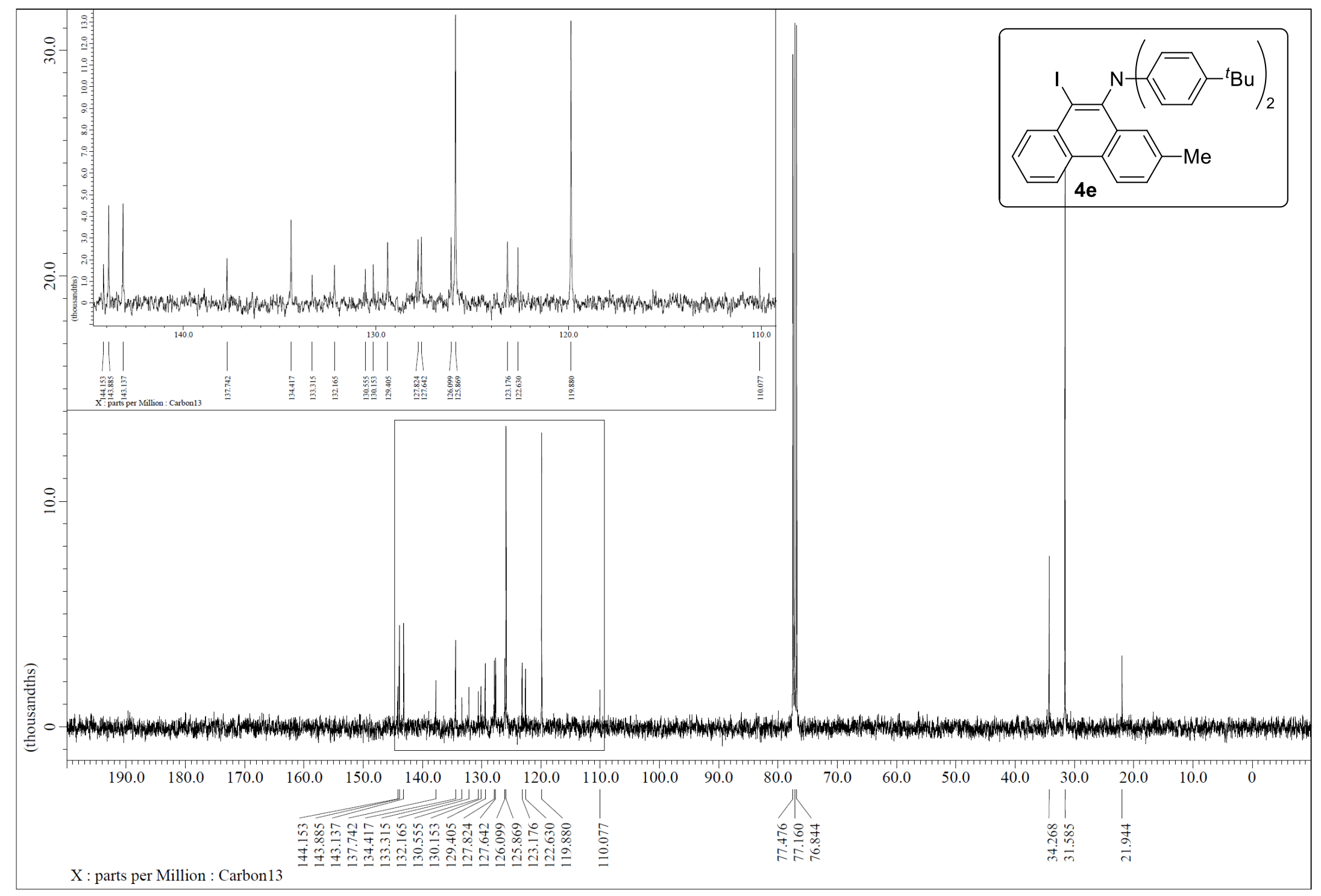

Figure S53. ${ }^{13} \mathrm{C}\left\{{ }^{1} \mathrm{H}\right\} \mathrm{NMR}(101 \mathrm{MHz})$ spectrum of $\mathbf{4 e}\left(\mathrm{CDCl}_{3}, \mathrm{rt}\right)$ 


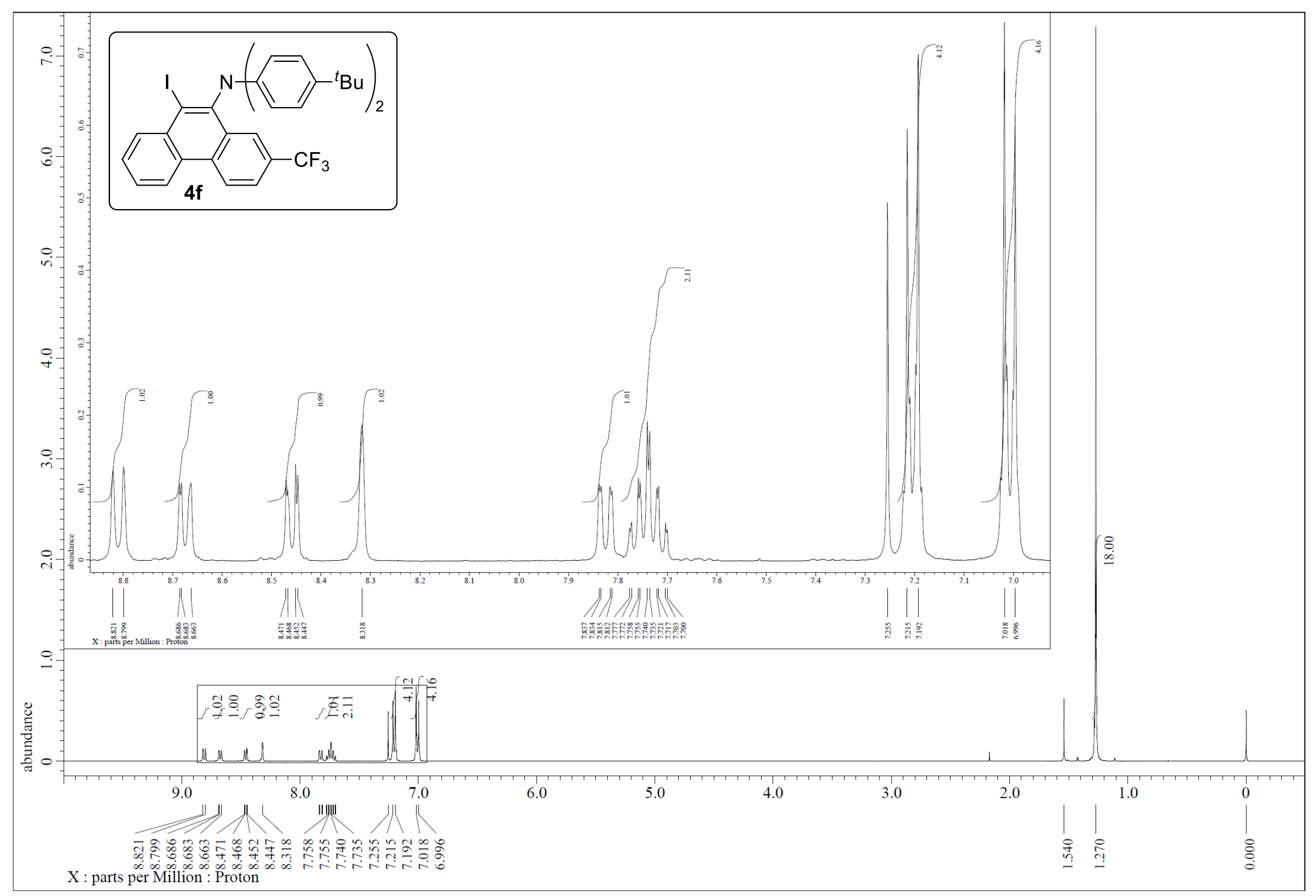

Figure S54. ${ }^{1} \mathrm{H}$ NMR (400 MHz) spectrum of $\mathbf{4 f}\left(\mathrm{CDCl}_{3}, \mathrm{rt}\right)$ 


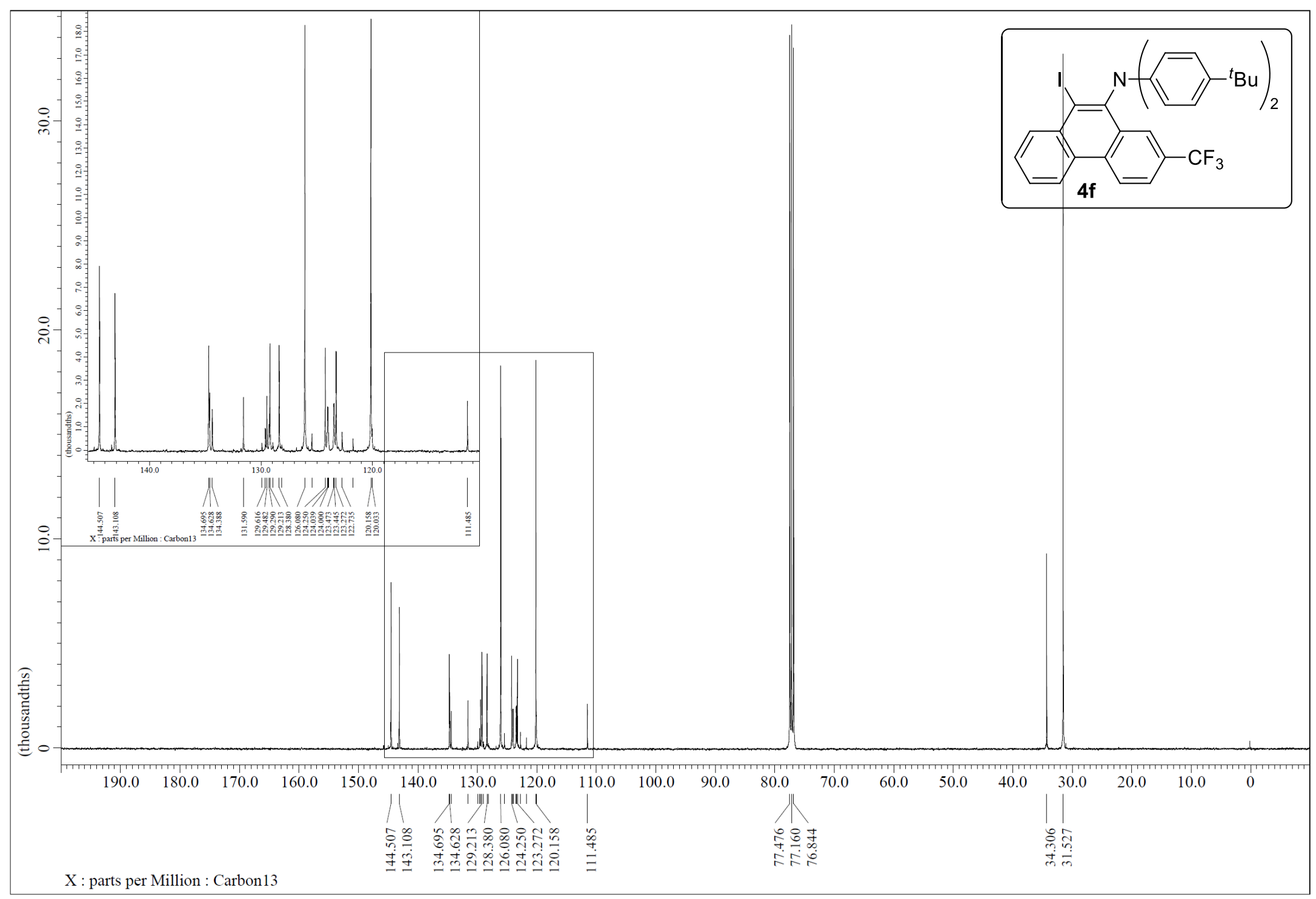

Figure S55. ${ }^{13} \mathrm{C}\left\{{ }^{1} \mathrm{H}\right\}$ NMR $(101 \mathrm{MHz})$ spectrum of $\mathbf{4} \mathbf{f}\left(\mathrm{CDCl}_{3}, \mathrm{rt}\right)$ 


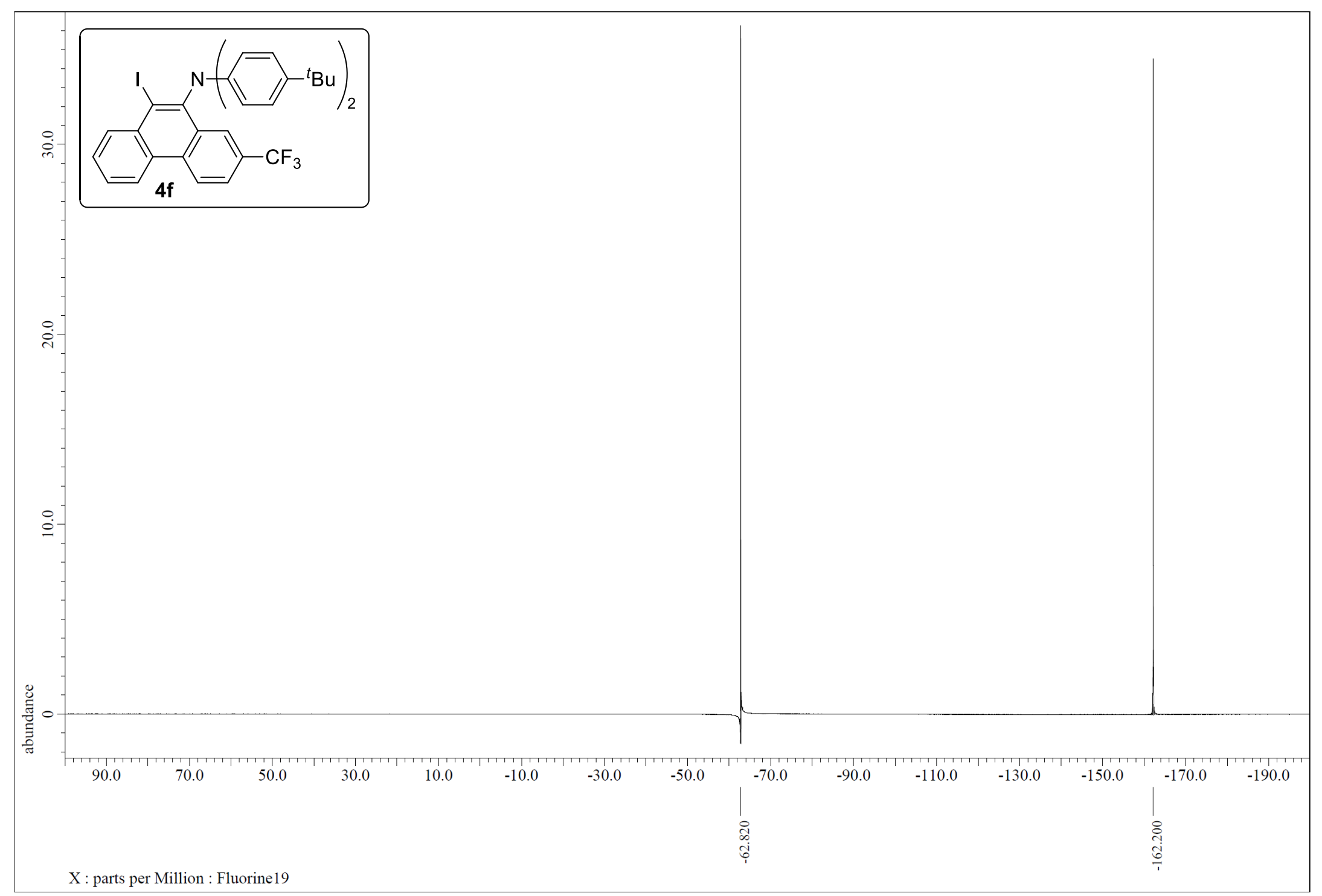

Figure S56. ${ }^{19} \mathrm{~F}\left\{{ }^{1} \mathrm{H}\right\}$ NMR $(376 \mathrm{MHz})$ spectrum of $\mathbf{4 f}\left(\mathrm{CDCl}_{3}, \mathrm{rt}\right)$ 


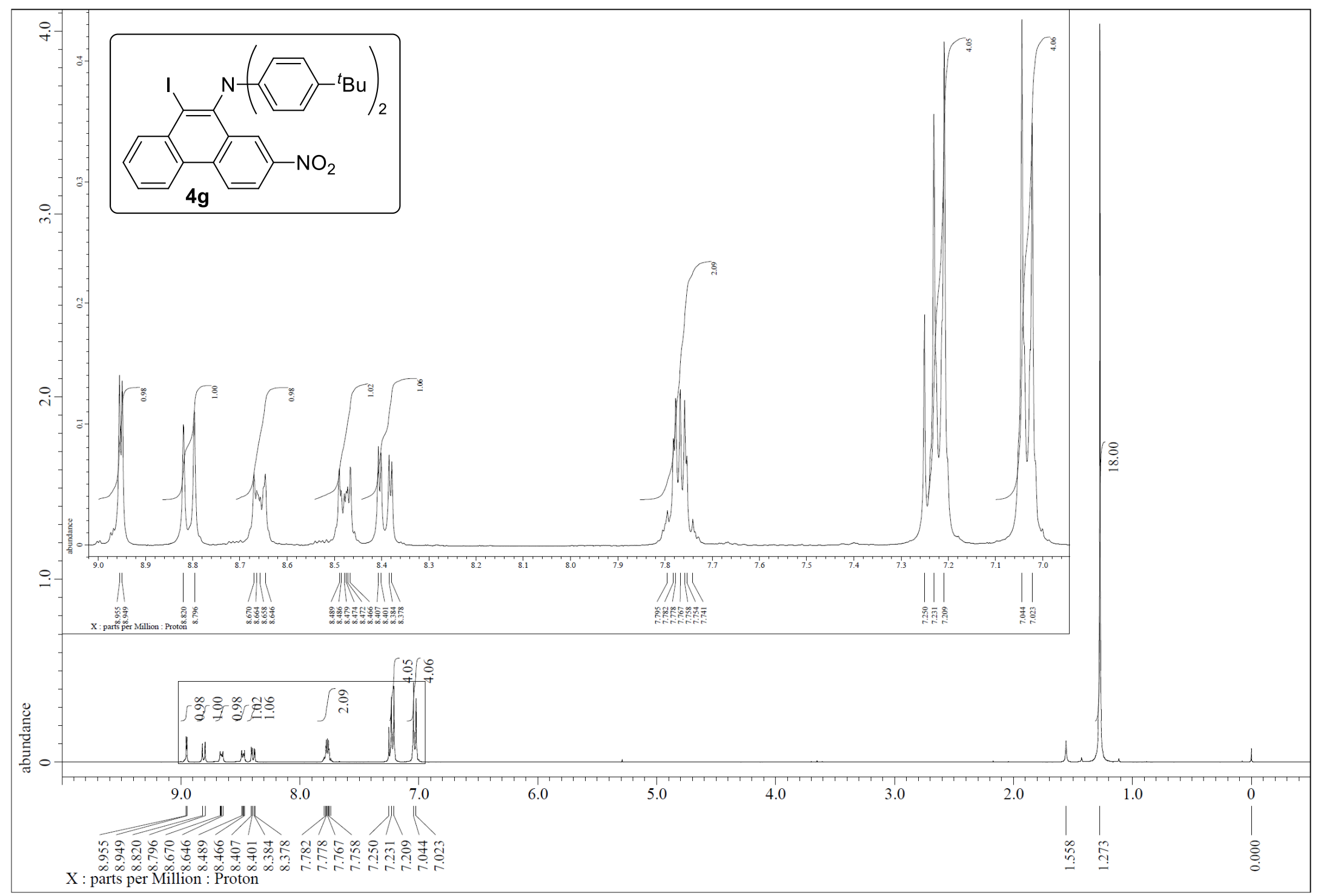

Figure S57. ${ }^{1} \mathrm{H}$ NMR $(400 \mathrm{MHz})$ spectrum of $\mathbf{4 g}\left(\mathrm{CDCl}_{3}, \mathrm{rt}\right)$ 


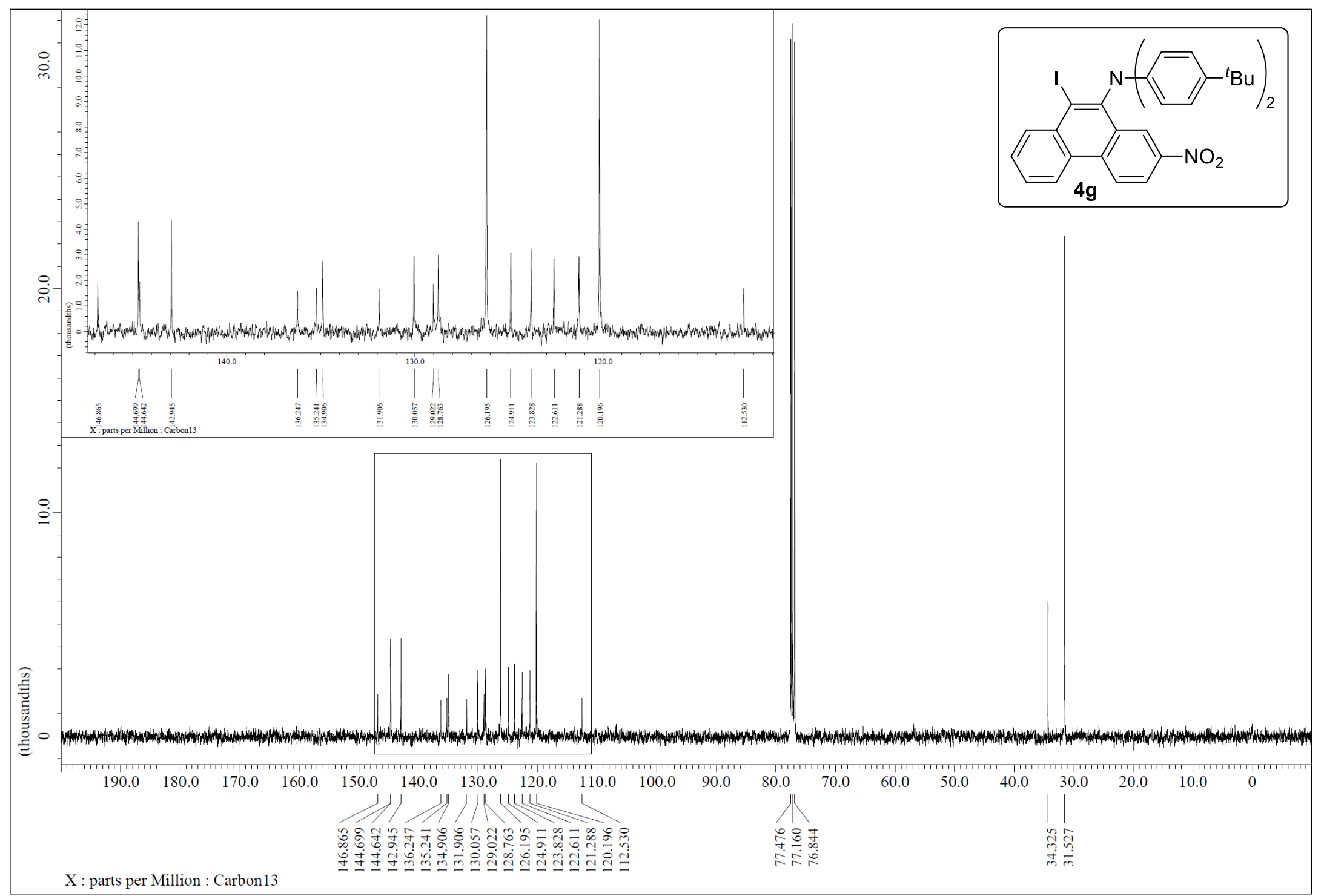

Figure S58. ${ }^{13} \mathrm{C}\left\{{ }^{1} \mathrm{H}\right\}$ NMR $(101 \mathrm{MHz})$ spectrum of $\mathbf{4 g}\left(\mathrm{CDCl}_{3}, \mathrm{rt}\right)$ 


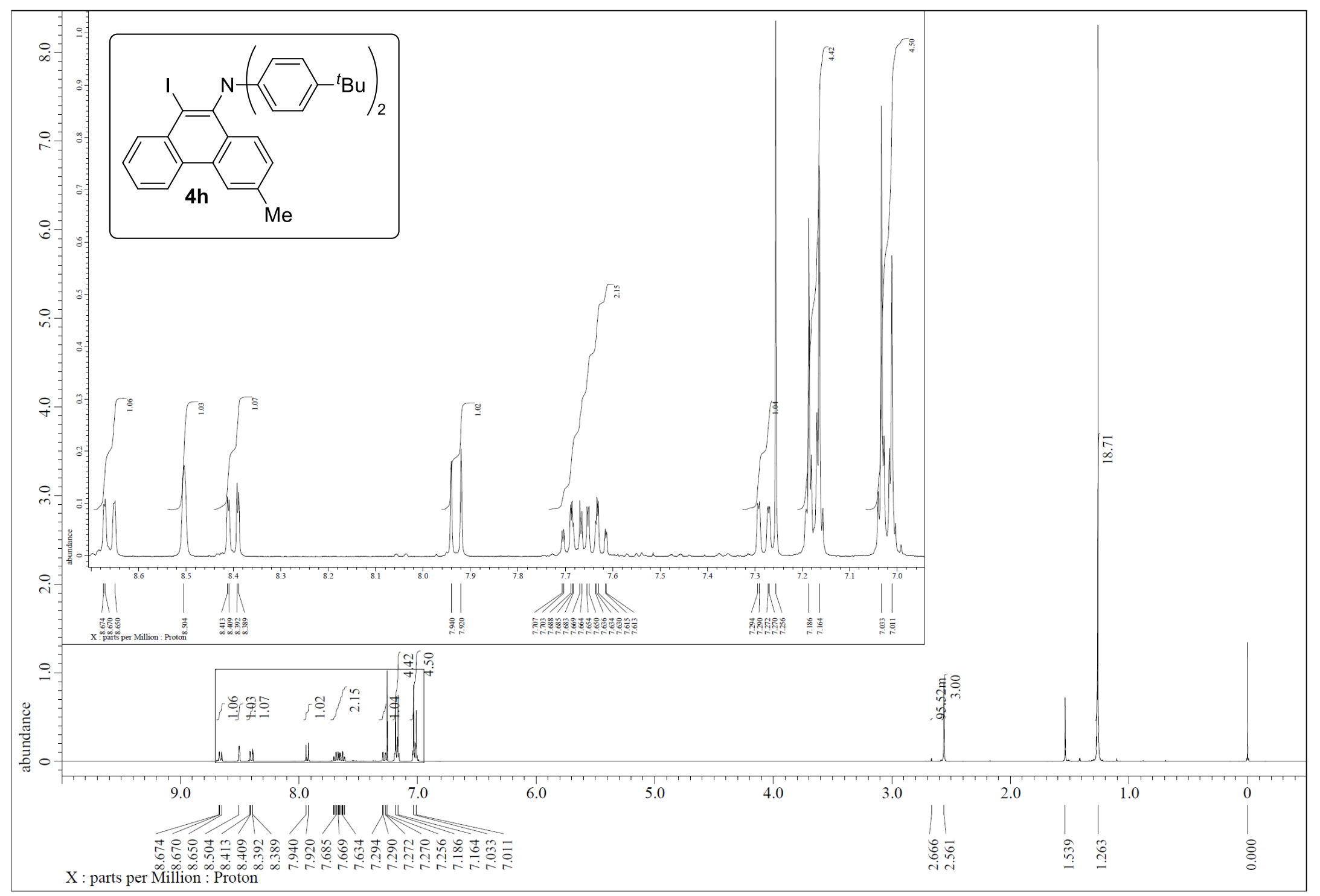

Figure S59. ${ }^{1} \mathrm{H}$ NMR $(400 \mathrm{MHz})$ spectrum of $\mathbf{4 h}\left(\mathrm{CDCl}_{3}, \mathrm{rt}\right)$ 


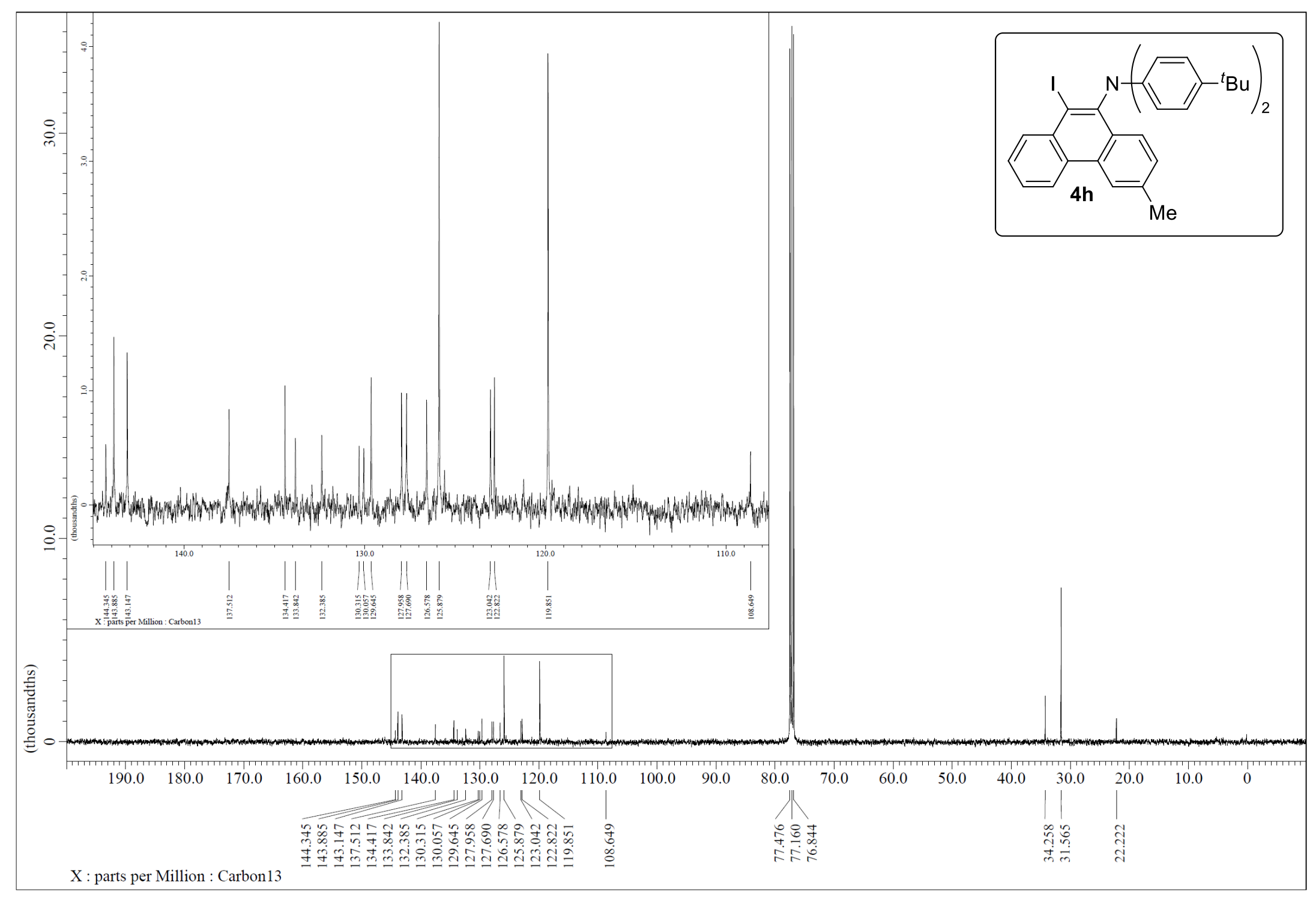

Figure S60. ${ }^{13} \mathrm{C}\left\{{ }^{1} \mathrm{H}\right\}$ NMR $(101 \mathrm{MHz})$ spectrum of $\mathbf{4 h}\left(\mathrm{CDCl}_{3}, \mathrm{rt}\right)$ 


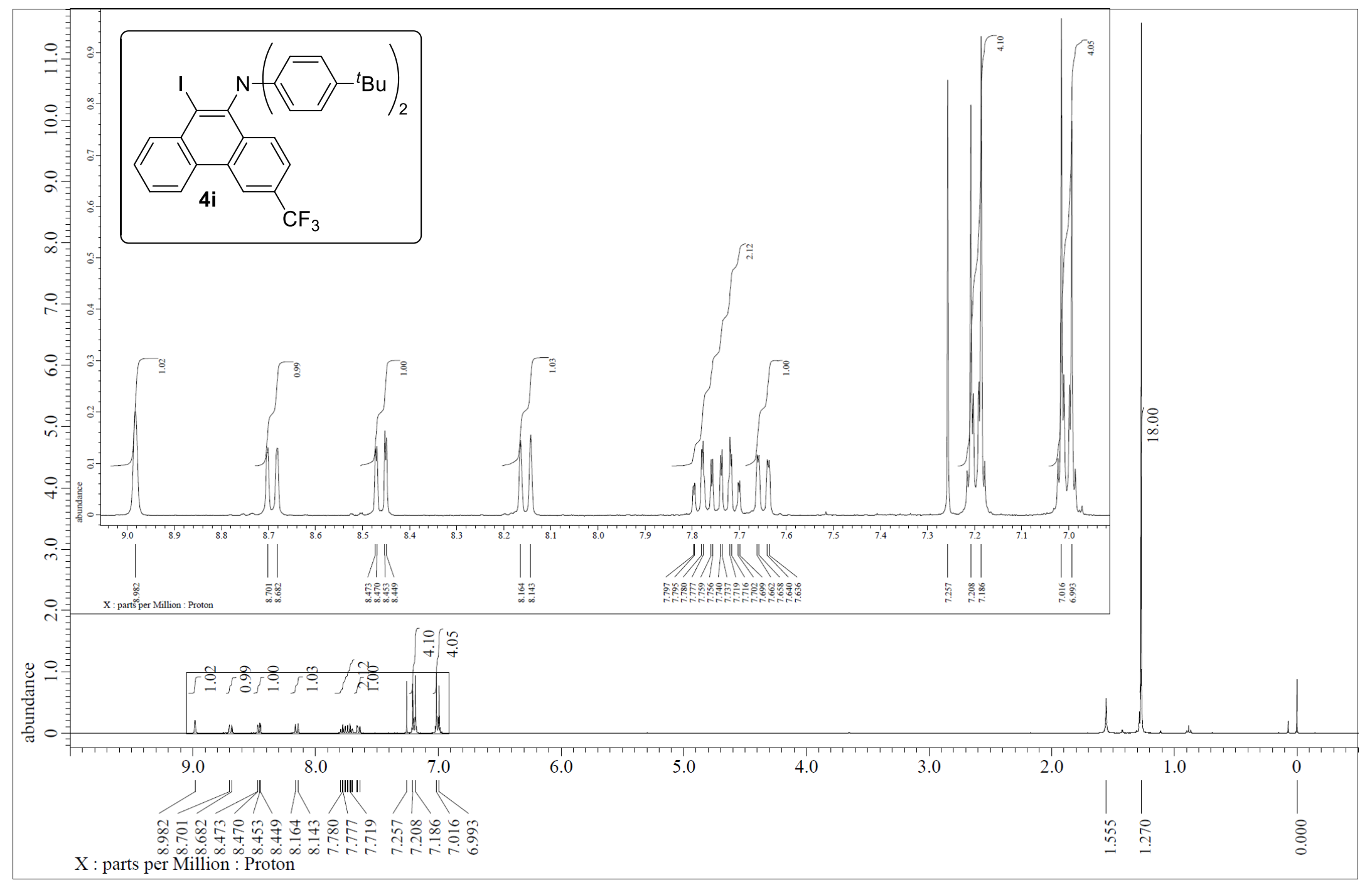

Figure S61. ${ }^{1} \mathrm{H}$ NMR (400 MHz) spectrum of $4 \mathbf{i}\left(\mathrm{CDCl}_{3}, \mathrm{rt}\right)$ 


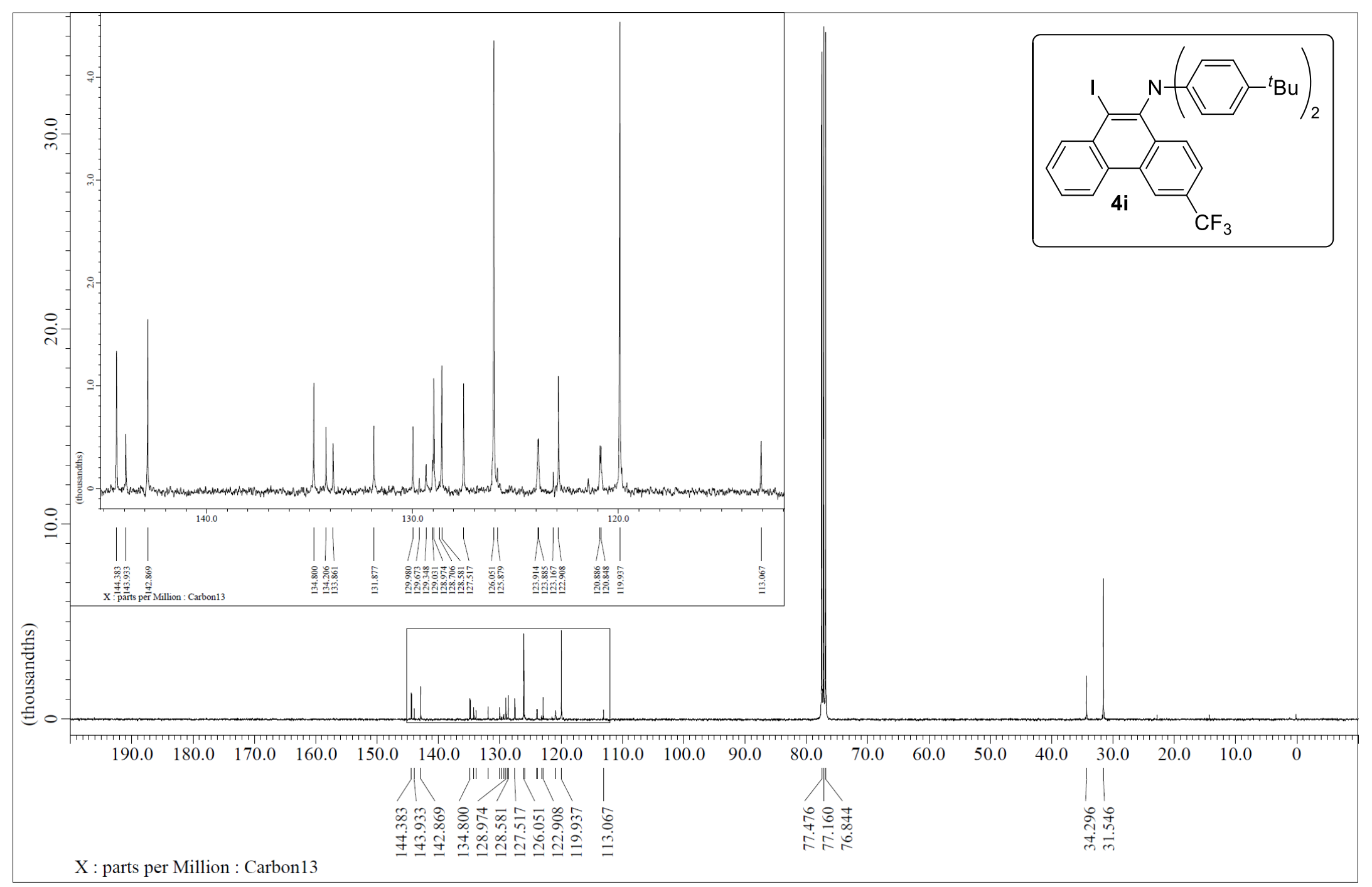

Figure S62. ${ }^{13} \mathrm{C}\left\{{ }^{1} \mathrm{H}\right\} \mathrm{NMR}(101 \mathrm{MHz})$ spectrum of $\mathbf{4 i}\left(\mathrm{CDCl}_{3}, \mathrm{rt}\right)$ 


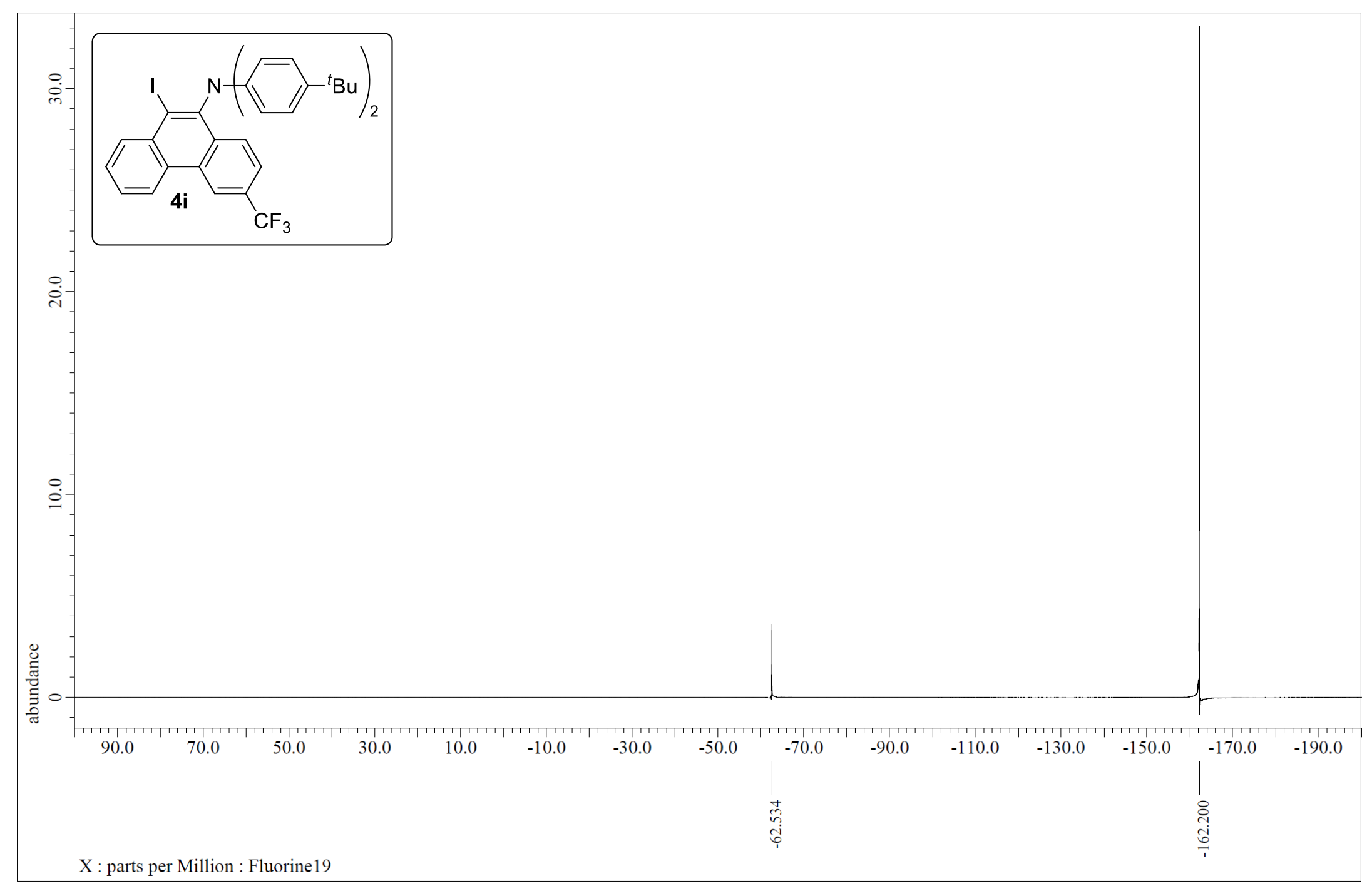

Figure S63. ${ }^{19} \mathrm{~F}\left\{{ }^{1} \mathrm{H}\right\}$ NMR $(376 \mathrm{MHz})$ spectrum of $\mathbf{4 i}\left(\mathrm{CDCl}_{3}\right.$, rt $)$ 


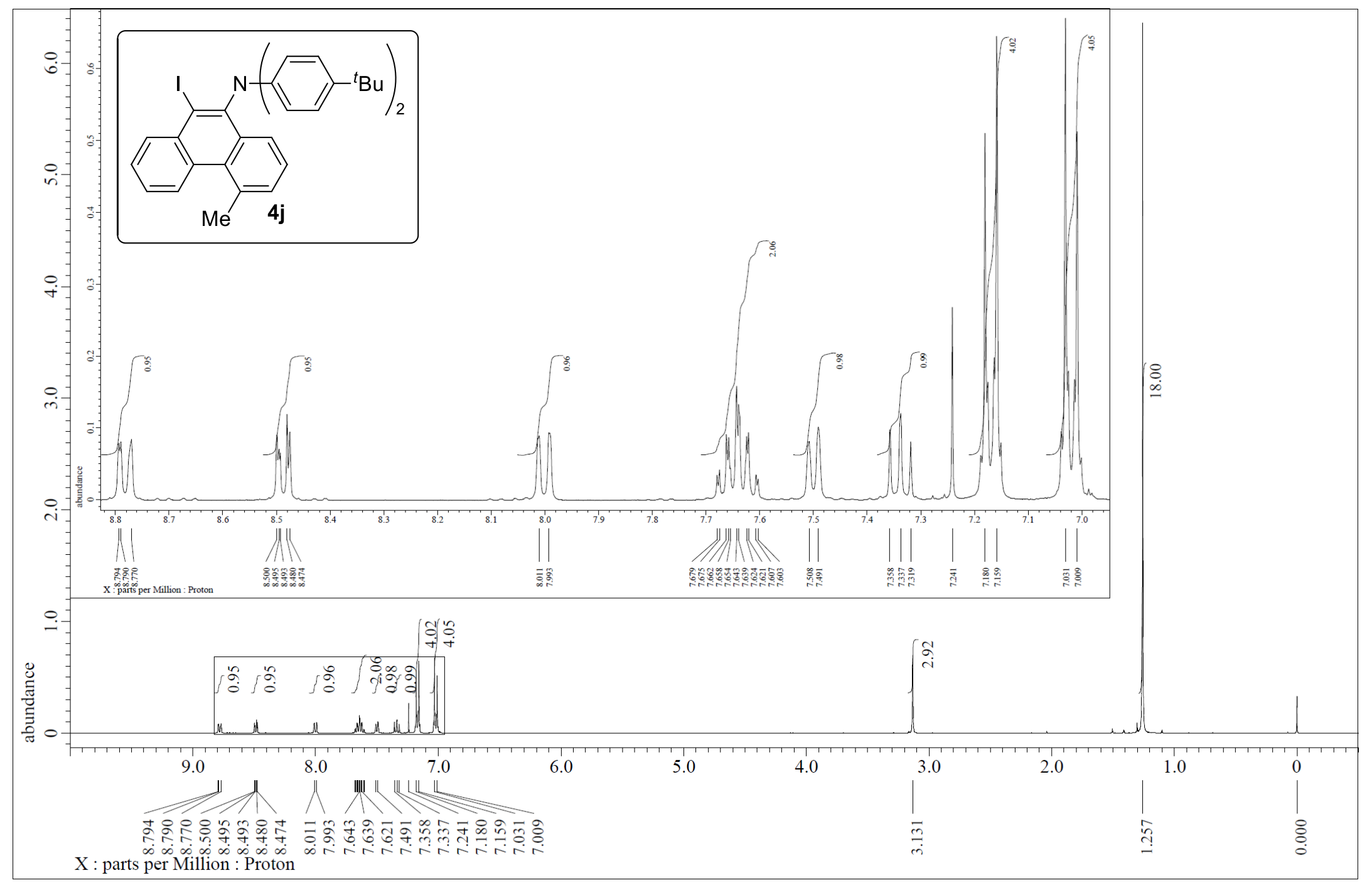

Figure S64. ${ }^{1} \mathrm{H}$ NMR $(400 \mathrm{MHz})$ spectrum of $\mathbf{4 j}\left(\mathrm{CDCl}_{3}, \mathrm{rt}\right)$ 


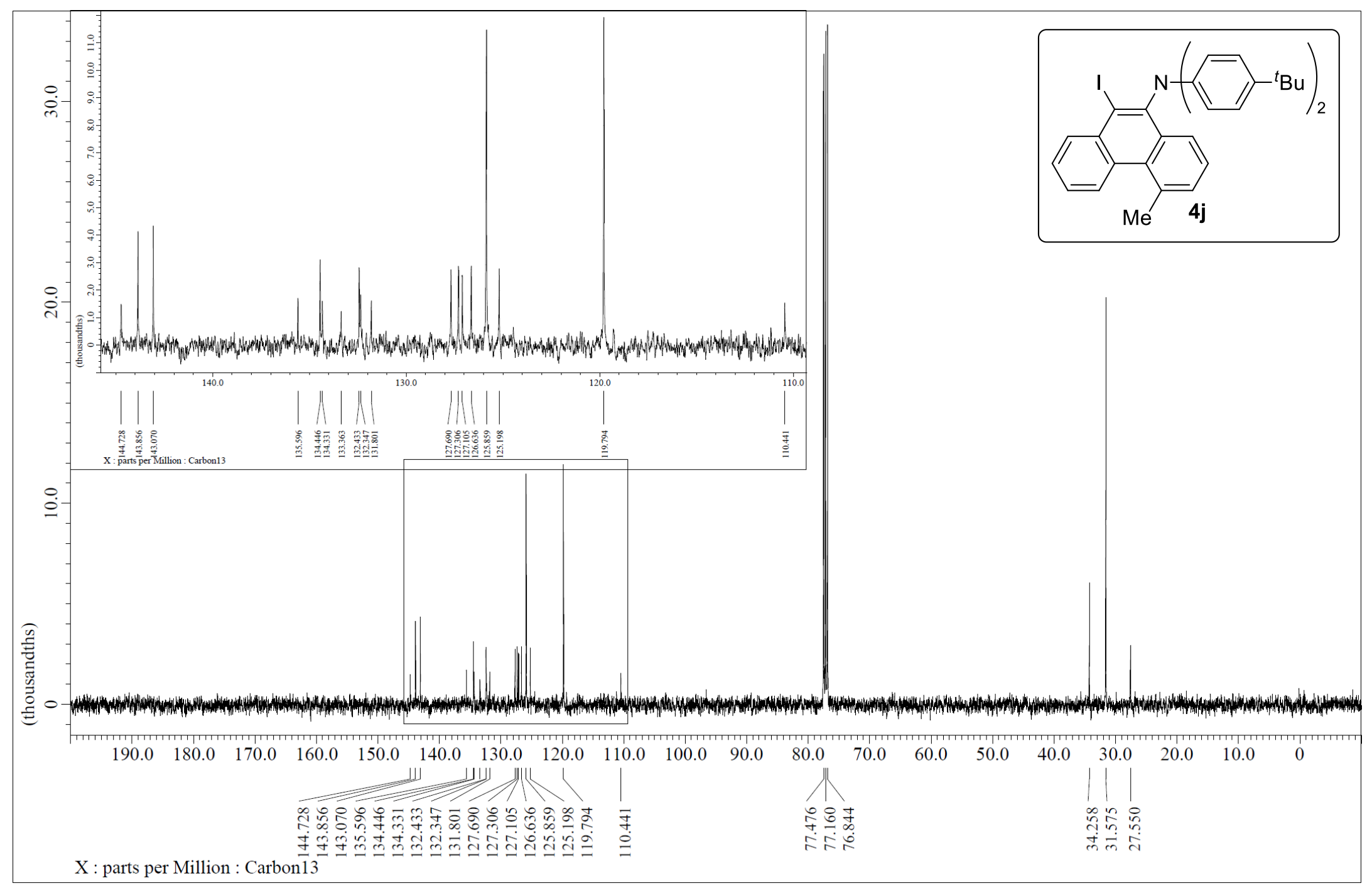

Figure S65. ${ }^{13} \mathrm{C}\left\{{ }^{1} \mathrm{H}\right\} \mathrm{NMR}(101 \mathrm{MHz})$ spectrum of $\mathbf{4 j}\left(\mathrm{CDCl}_{3}, \mathrm{rt}\right)$ 


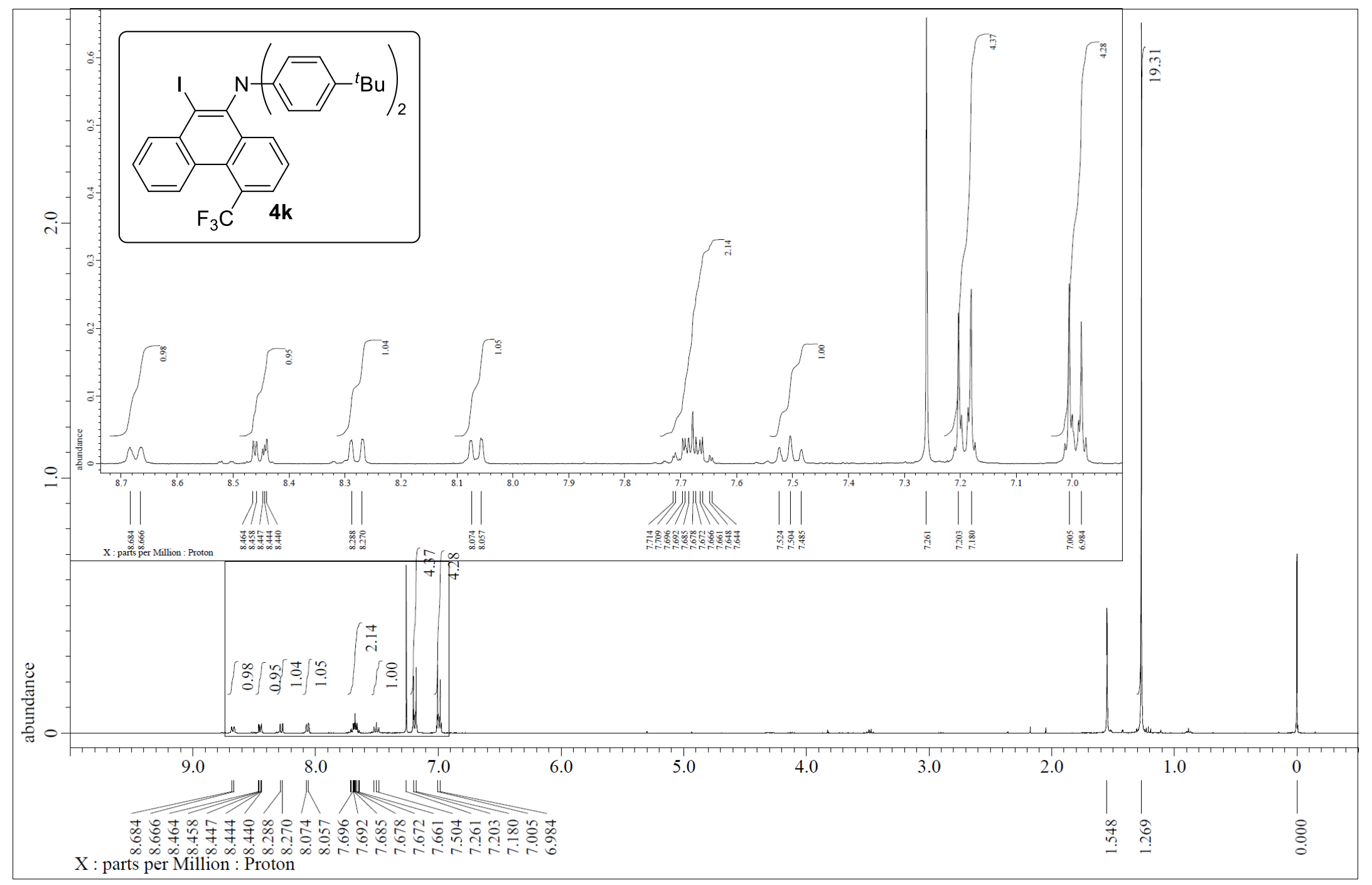

Figure S66. ${ }^{1} \mathrm{H}$ NMR $(400 \mathrm{MHz})$ spectrum of $\mathbf{4 k}\left(\mathrm{CDCl}_{3}, \mathrm{rt}\right)$ 


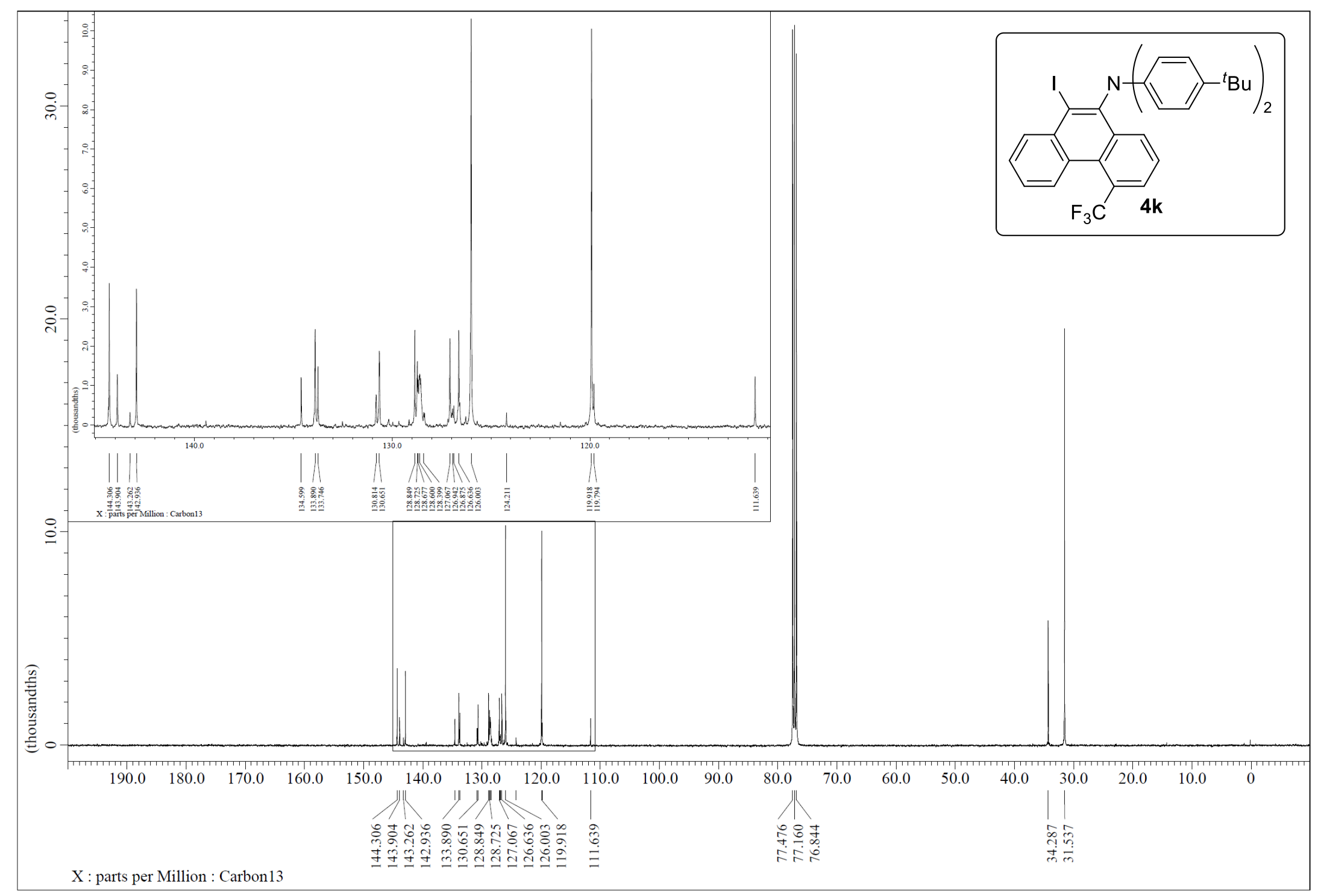

Figure S67. ${ }^{13} \mathrm{C}\left\{{ }^{1} \mathrm{H}\right\} \mathrm{NMR}(101 \mathrm{MHz})$ spectrum of $\mathbf{4 k}\left(\mathrm{CDCl}_{3}, \mathrm{rt}\right)$ 


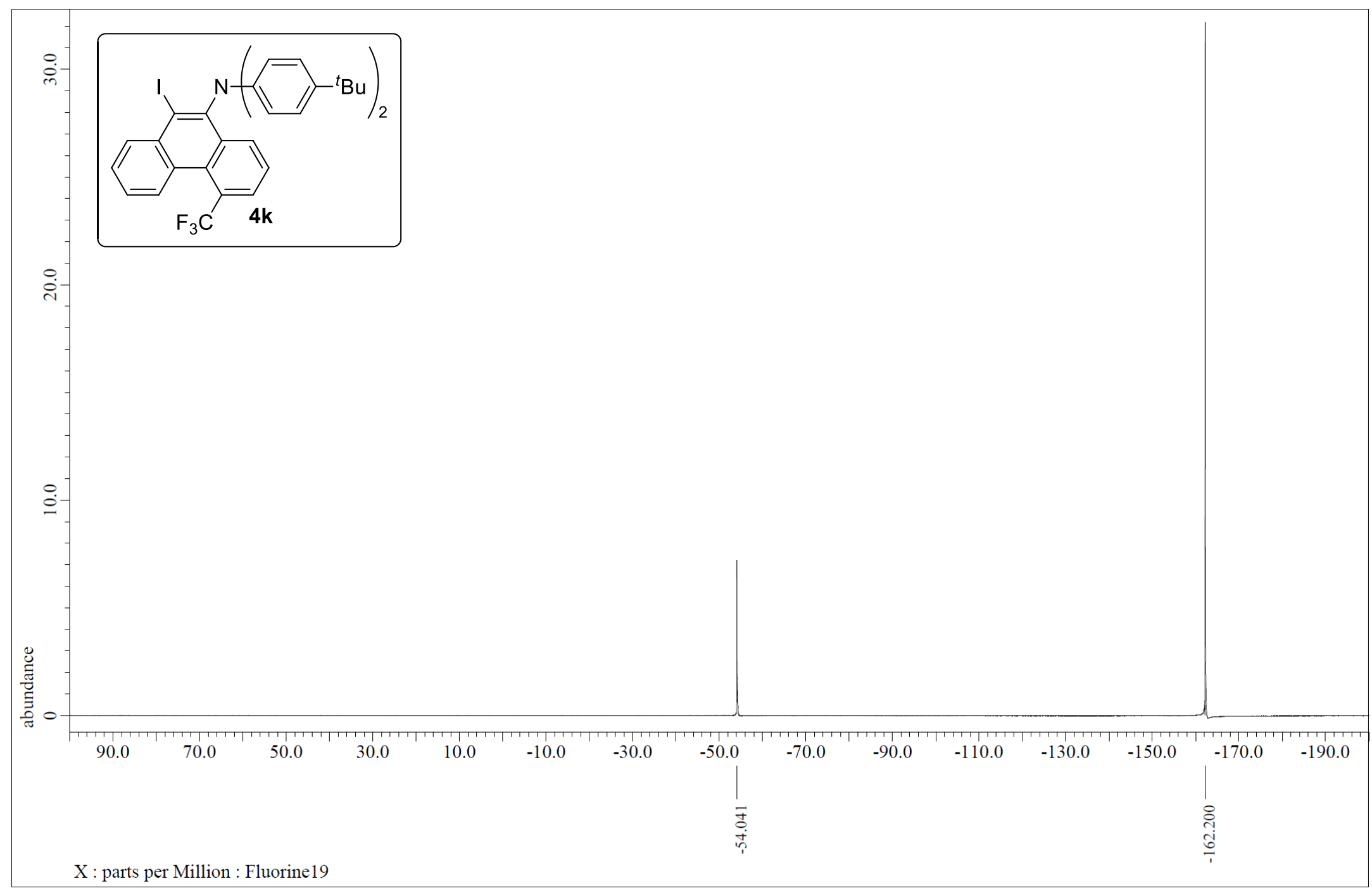

Figure S68. ${ }^{19} \mathrm{~F}\left\{{ }^{1} \mathrm{H}\right\}$ NMR (376 MHz) spectrum of $\mathbf{4 k}\left(\mathrm{CDCl}_{3}, \mathrm{rt}\right)$ 


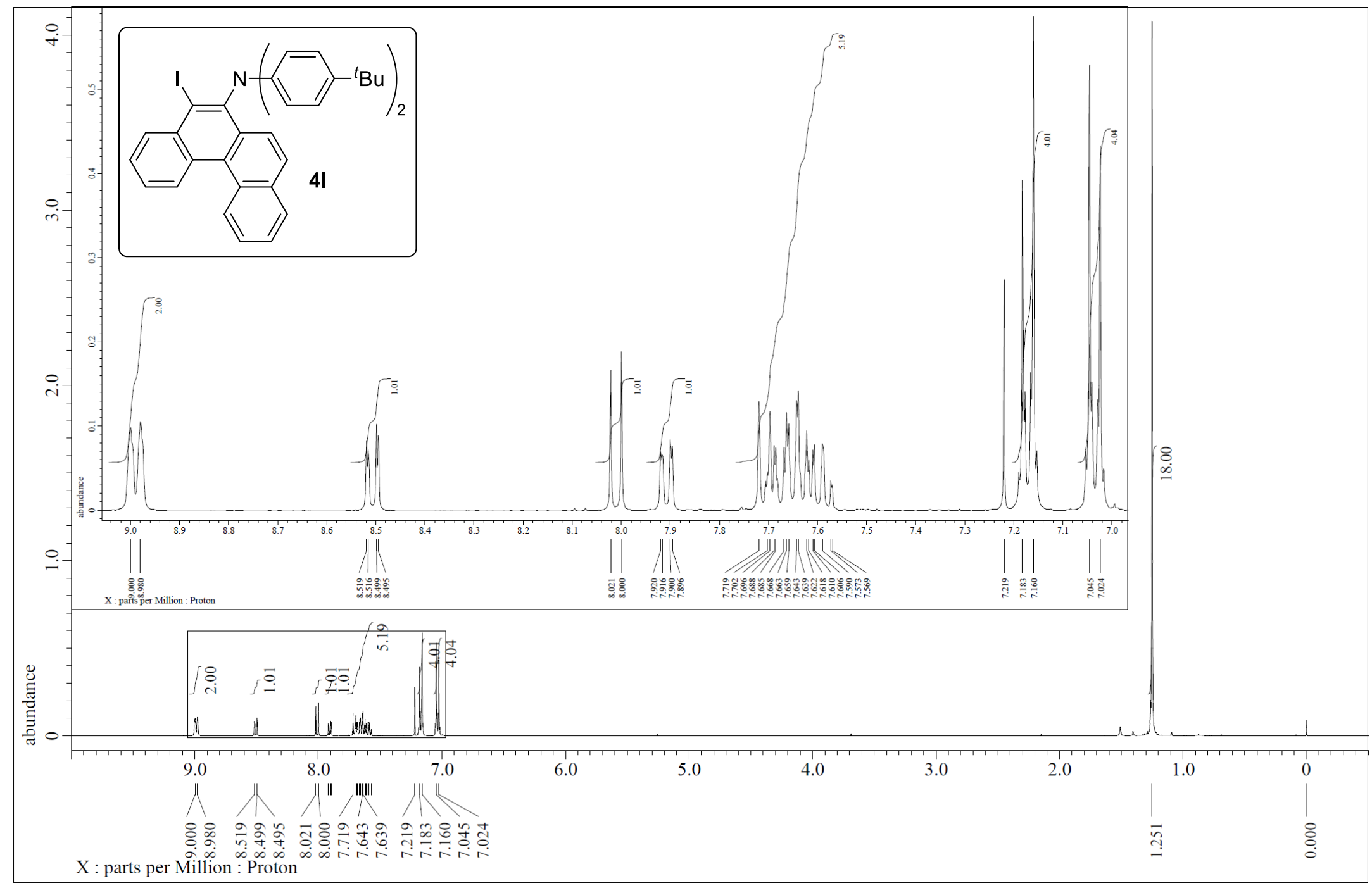

Figure S69. ${ }^{1} \mathrm{H}$ NMR $(400 \mathrm{MHz})$ spectrum of $\mathbf{4 l}\left(\mathrm{CDCl}_{3}, \mathrm{rt}\right)$ 


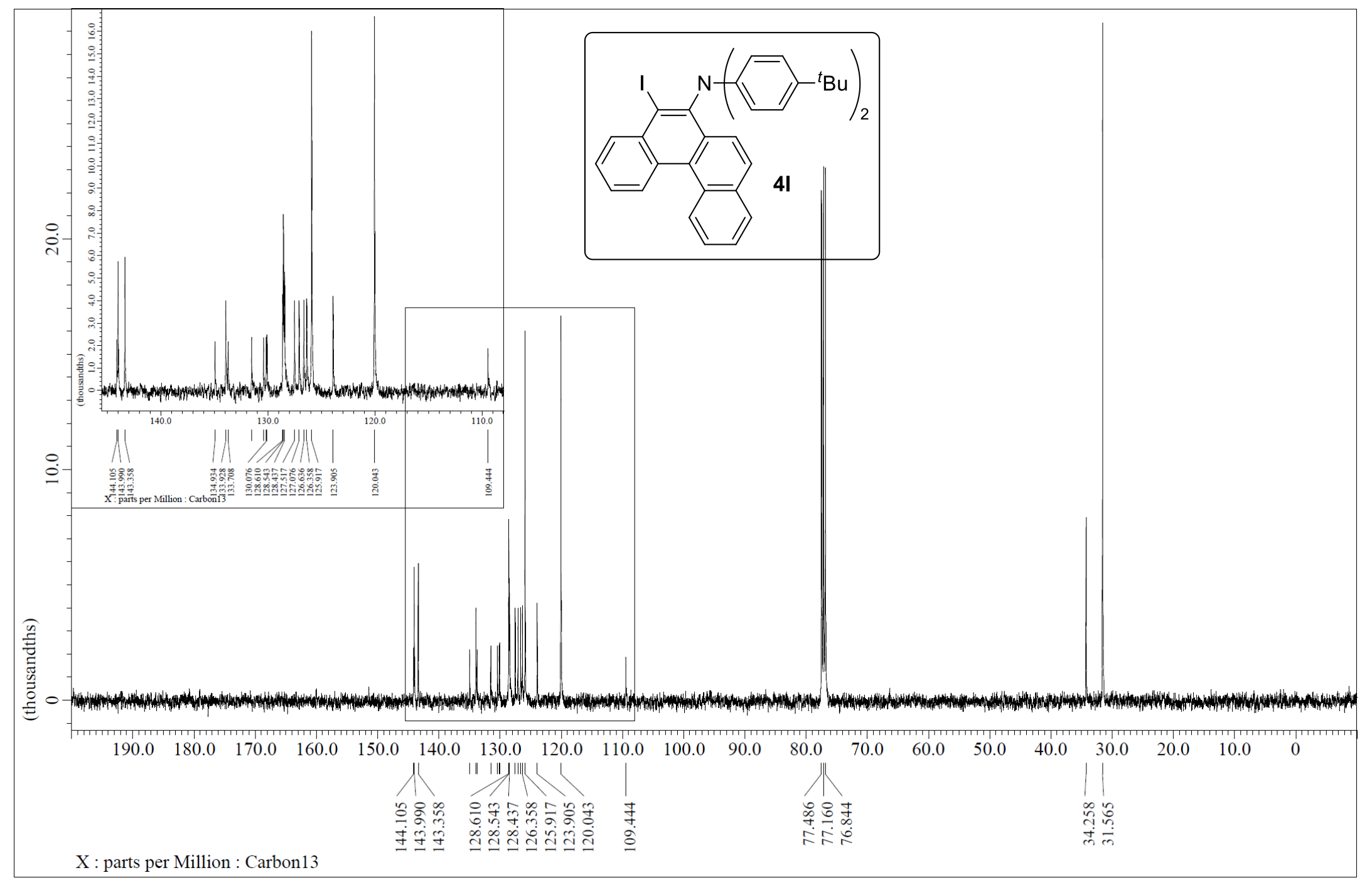

Figure S70. ${ }^{13} \mathrm{C}\left\{{ }^{1} \mathrm{H}\right\}$ NMR $(101 \mathrm{MHz})$ spectrum of $\mathbf{4 l}\left(\mathrm{CDCl}_{3}, \mathrm{rt}\right)$ 


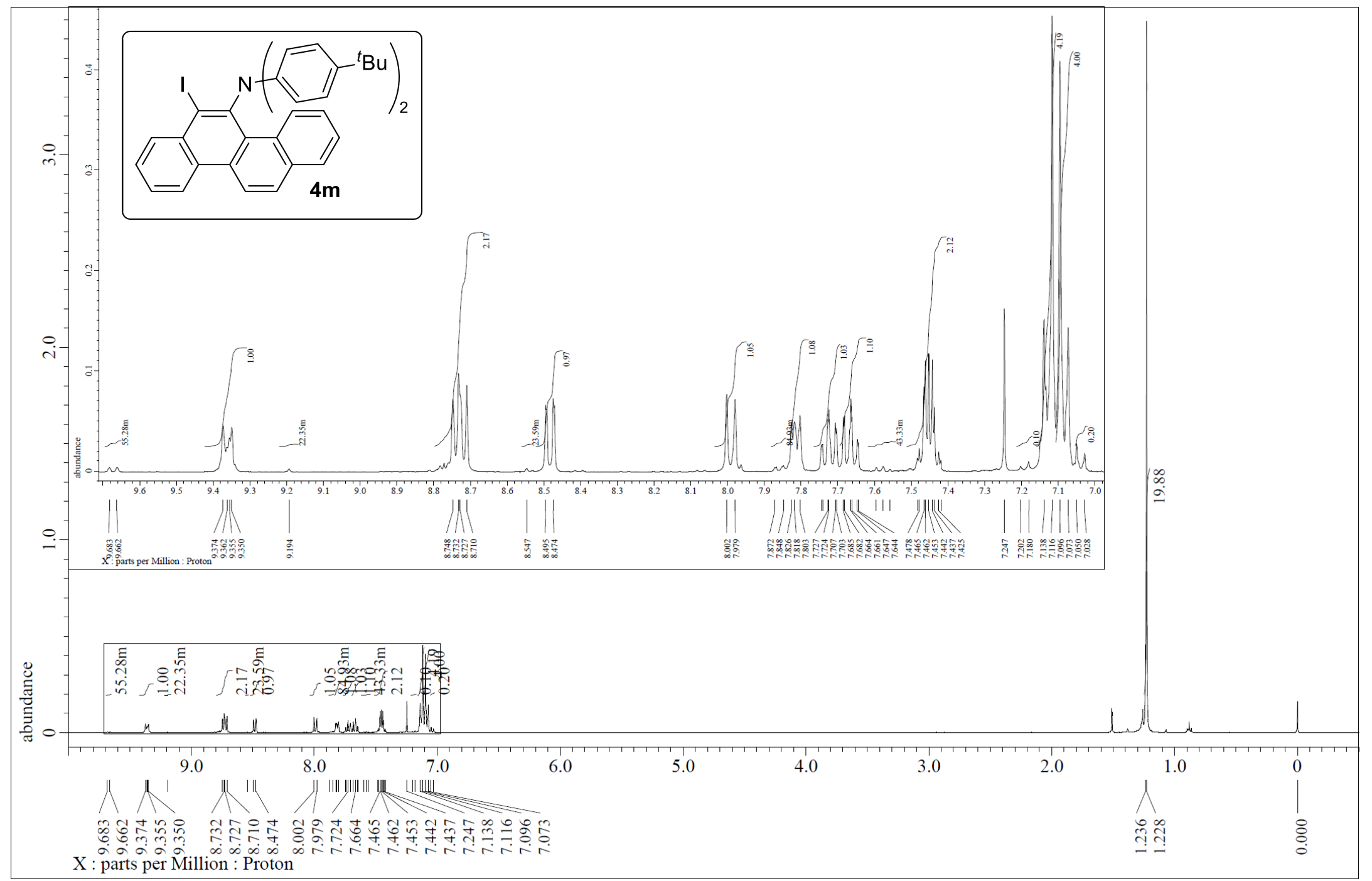

Figure S71. ${ }^{1} \mathrm{H}$ NMR $(400 \mathrm{MHz})$ spectrum of $\mathbf{4 m}\left(\mathrm{CDCl}_{3}, \mathrm{rt}\right)$ 


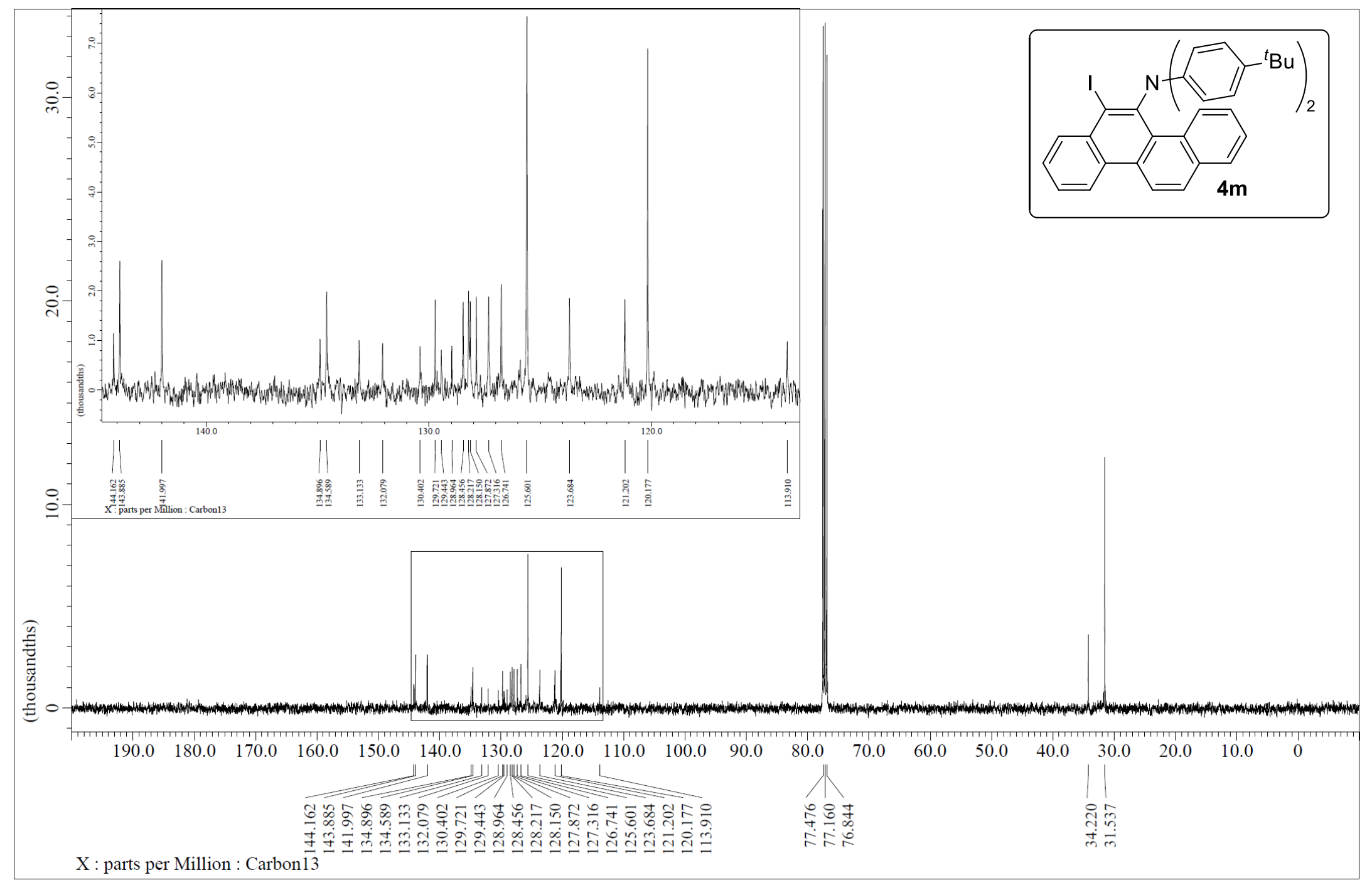

Figure S72. ${ }^{13} \mathrm{C}\left\{{ }^{1} \mathrm{H}\right\}$ NMR $(101 \mathrm{MHz})$ spectrum of $\mathbf{4 m}\left(\mathrm{CDCl}_{3}, \mathrm{rt}\right)$ 


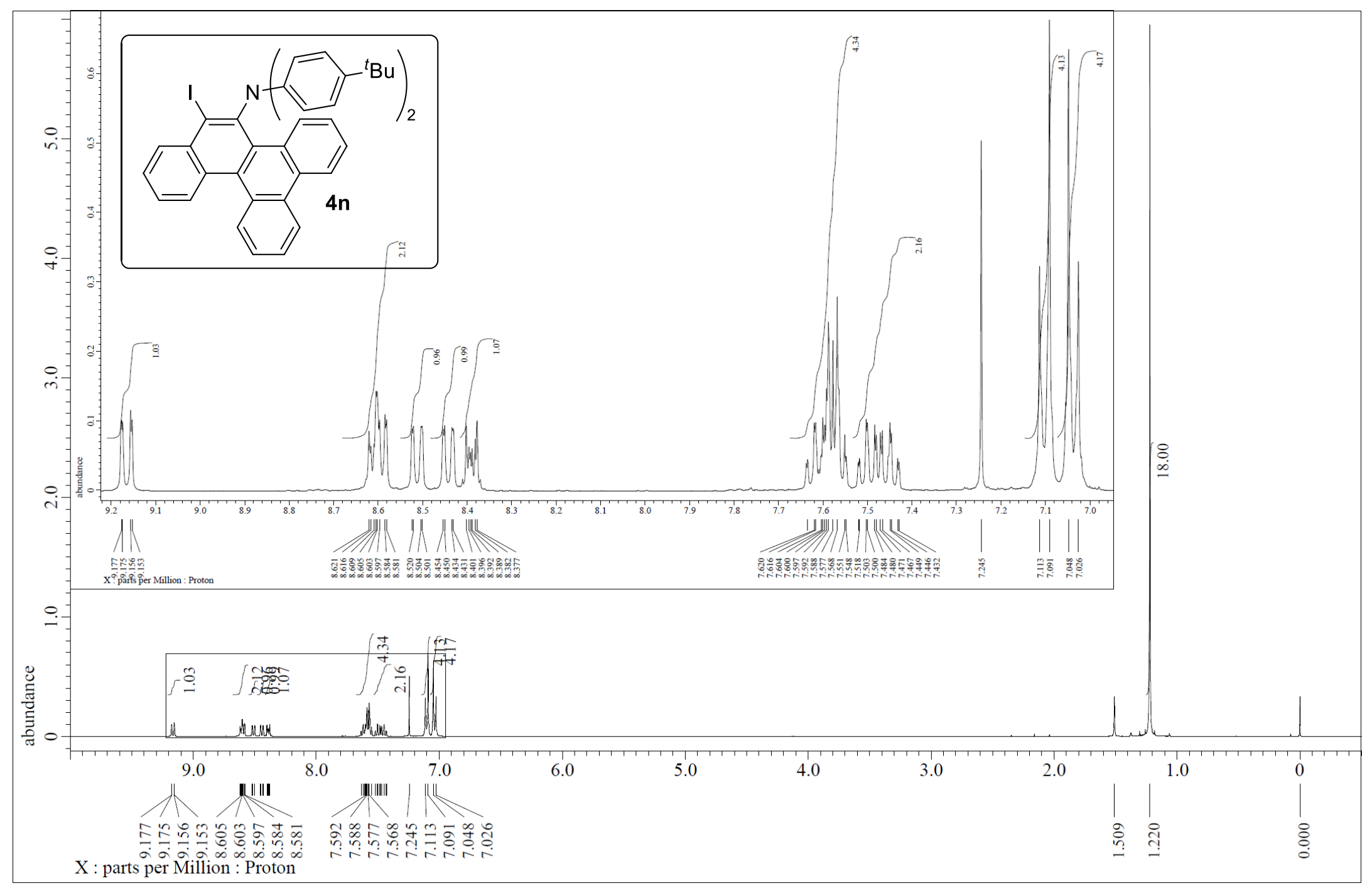

Figure S73. ${ }^{1} \mathrm{H}$ NMR (400 MHz) spectrum of $4 \mathbf{n}\left(\mathrm{CDCl}_{3}, \mathrm{rt}\right)$ 


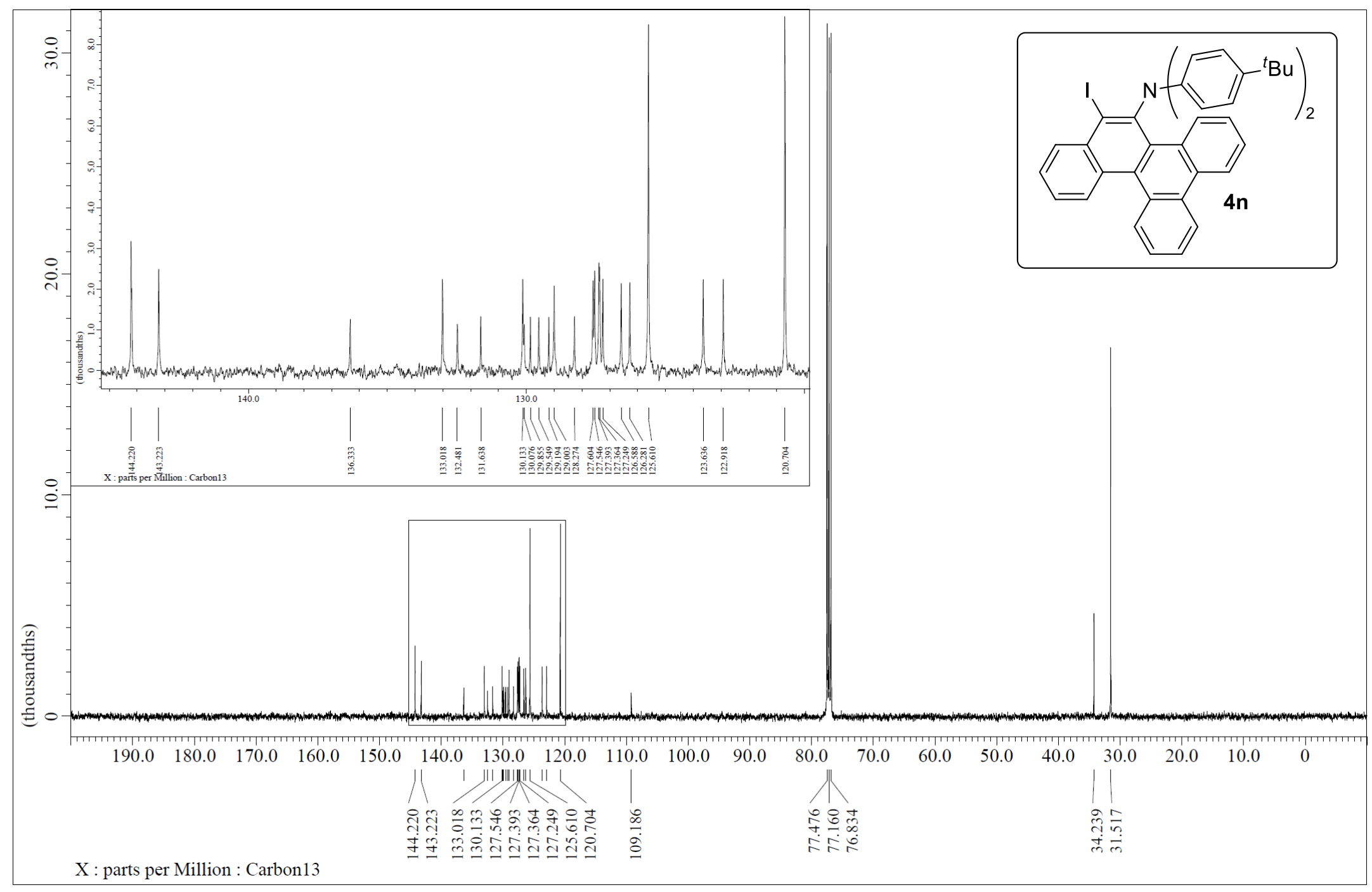

Figure S74. ${ }^{13} \mathrm{C}\left\{{ }^{1} \mathrm{H}\right\}$ NMR $(101 \mathrm{MHz})$ spectrum of $\mathbf{4 n}\left(\mathrm{CDCl}_{3}, \mathrm{rt}\right)$ 


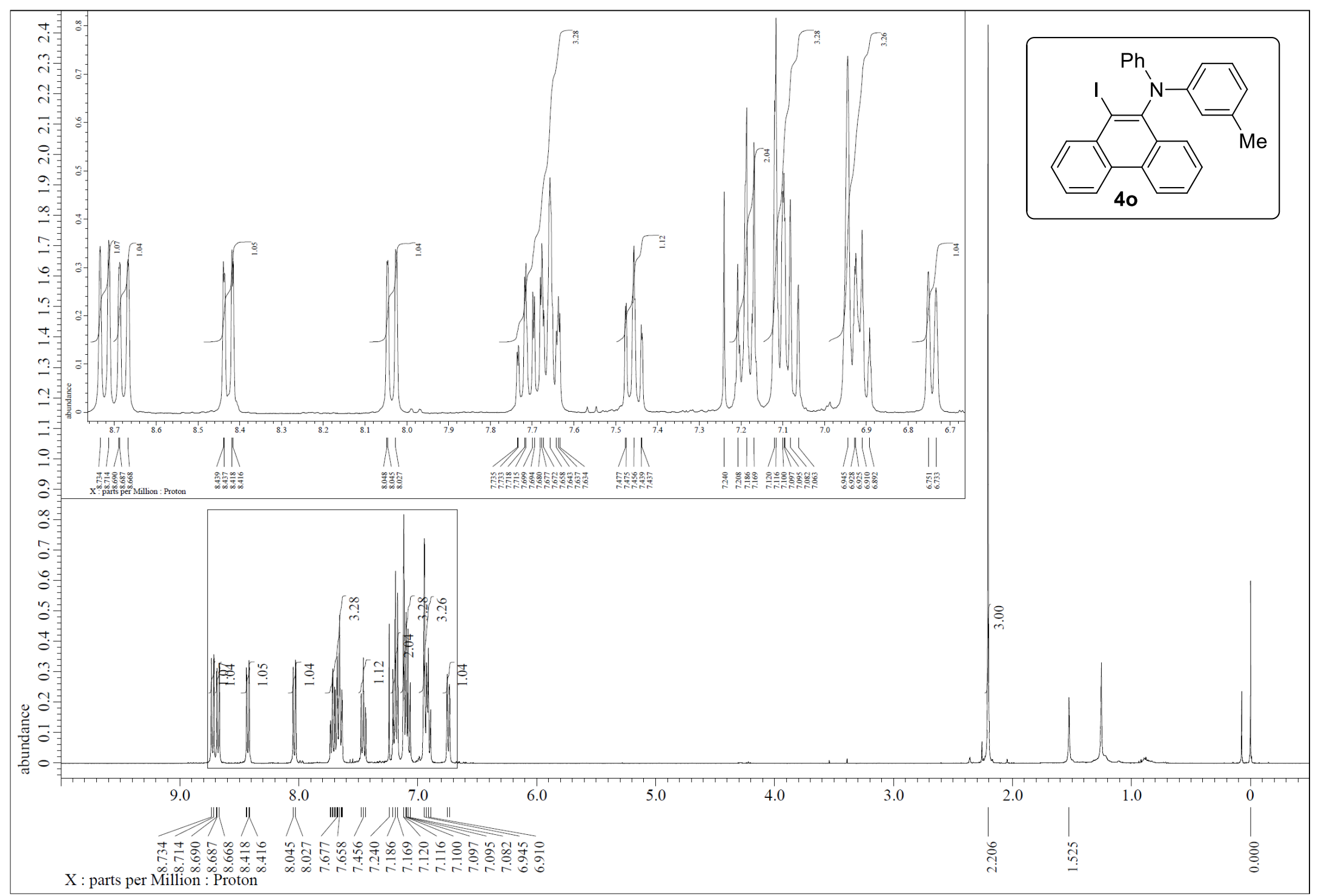

Figure S75. ${ }^{1} \mathrm{H}$ NMR $(400 \mathrm{MHz})$ spectrum of $4 \mathbf{o}\left(\mathrm{CDCl}_{3}, \mathrm{rt}\right)$ 


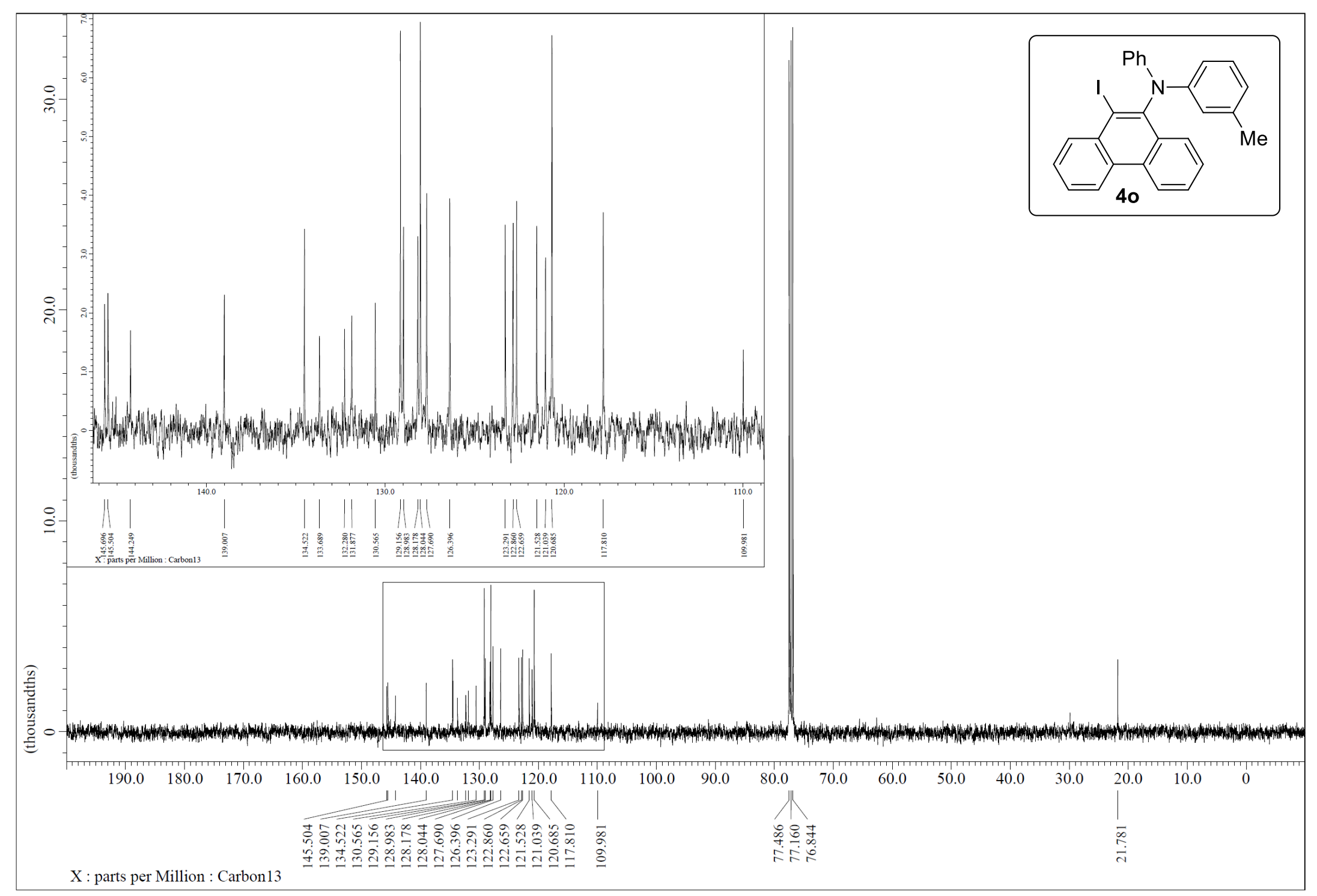

Figure S76. ${ }^{13} \mathrm{C}\left\{{ }^{1} \mathrm{H}\right\}$ NMR $(101 \mathrm{MHz})$ spectrum of $\mathbf{4 o}\left(\mathrm{CDCl}_{3}, \mathrm{rt}\right)$ 


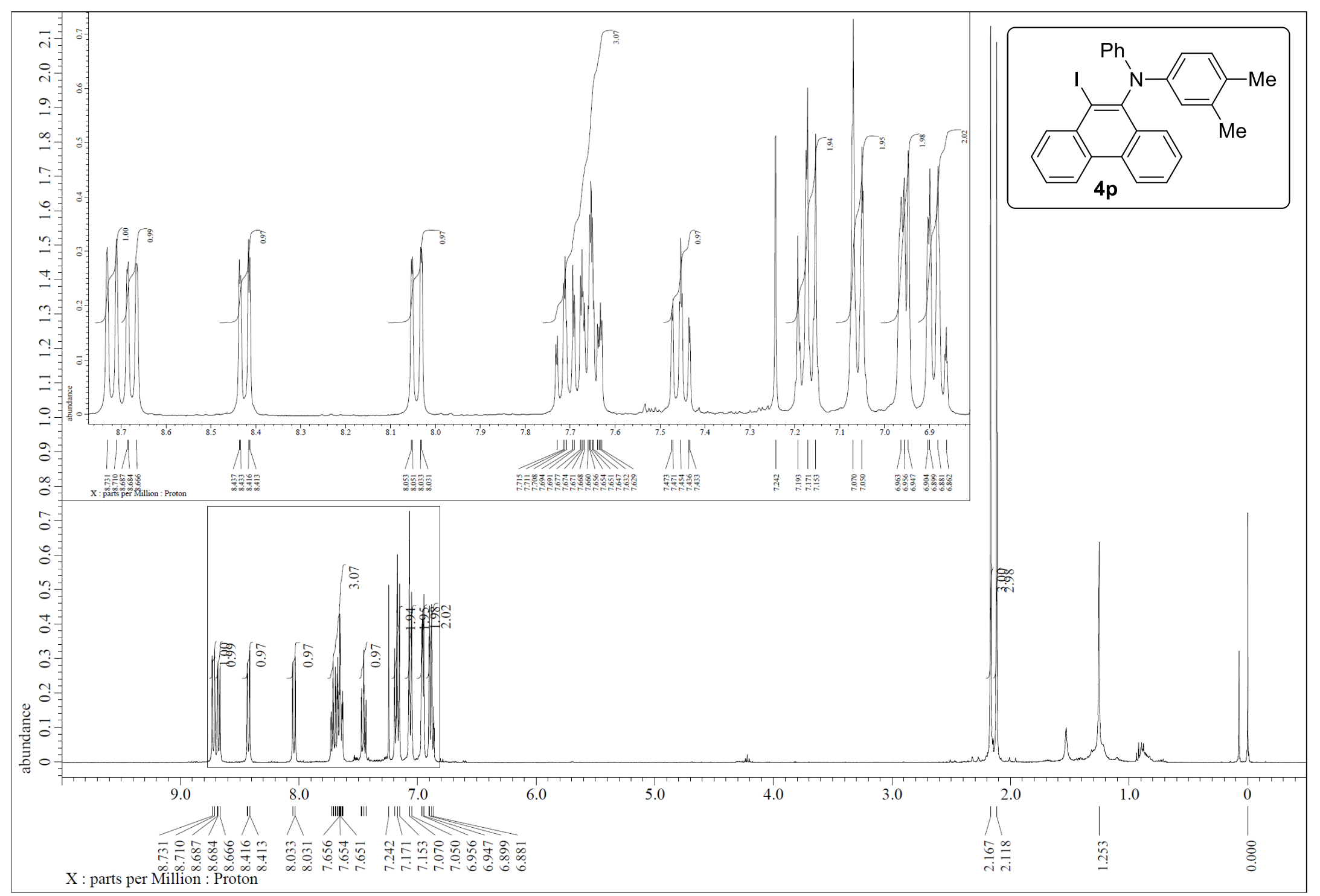

Figure S77. ${ }^{1} \mathrm{H}$ NMR (400 MHz) spectrum of $\mathbf{4 p}\left(\mathrm{CDCl}_{3}, \mathrm{rt}\right)$ 


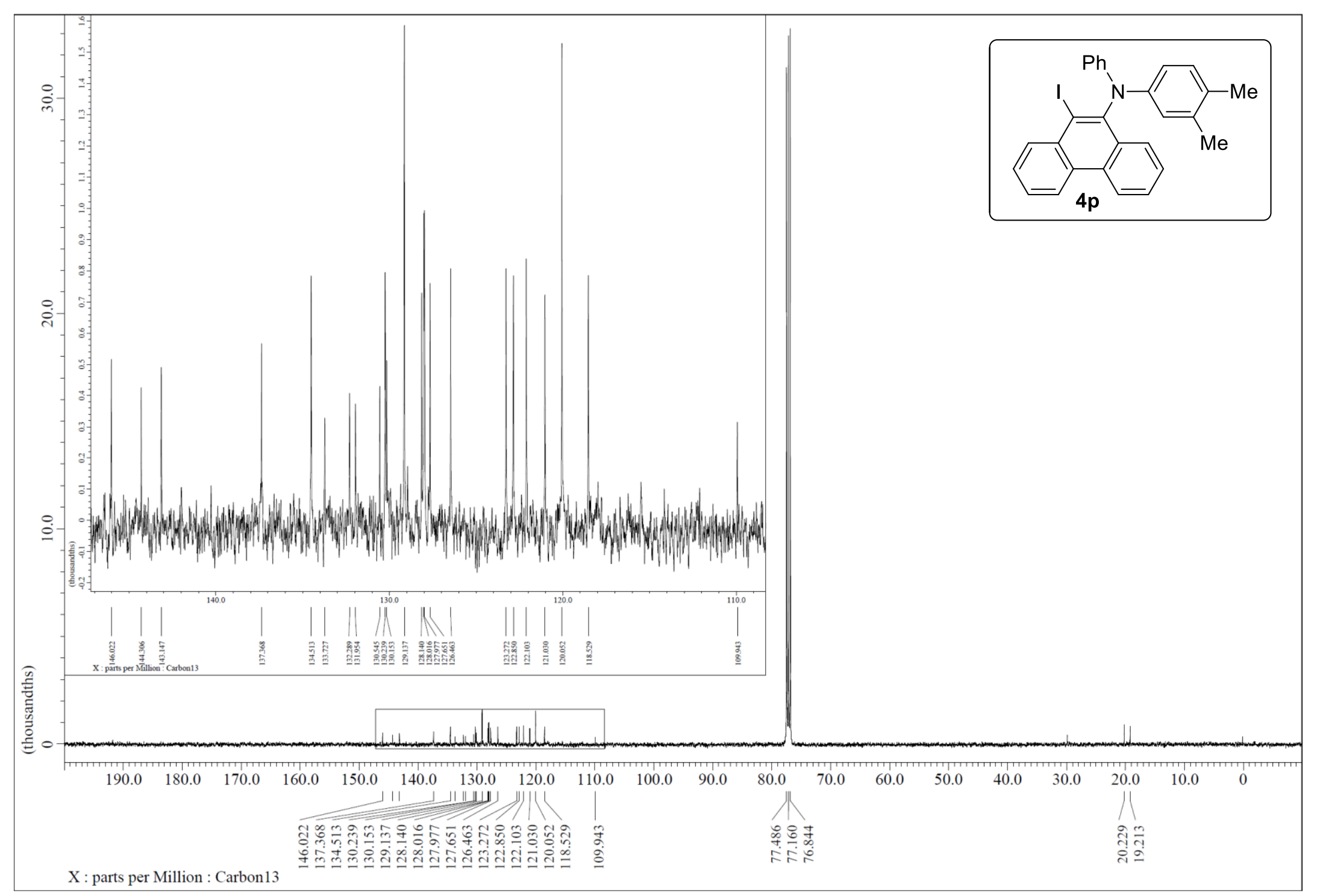

Figure S78. ${ }^{13} \mathrm{C}\left\{{ }^{1} \mathrm{H}\right\} \mathrm{NMR}(101 \mathrm{MHz})$ spectrum of $\mathbf{4 p}\left(\mathrm{CDCl}_{3}, \mathrm{rt}\right)$ 


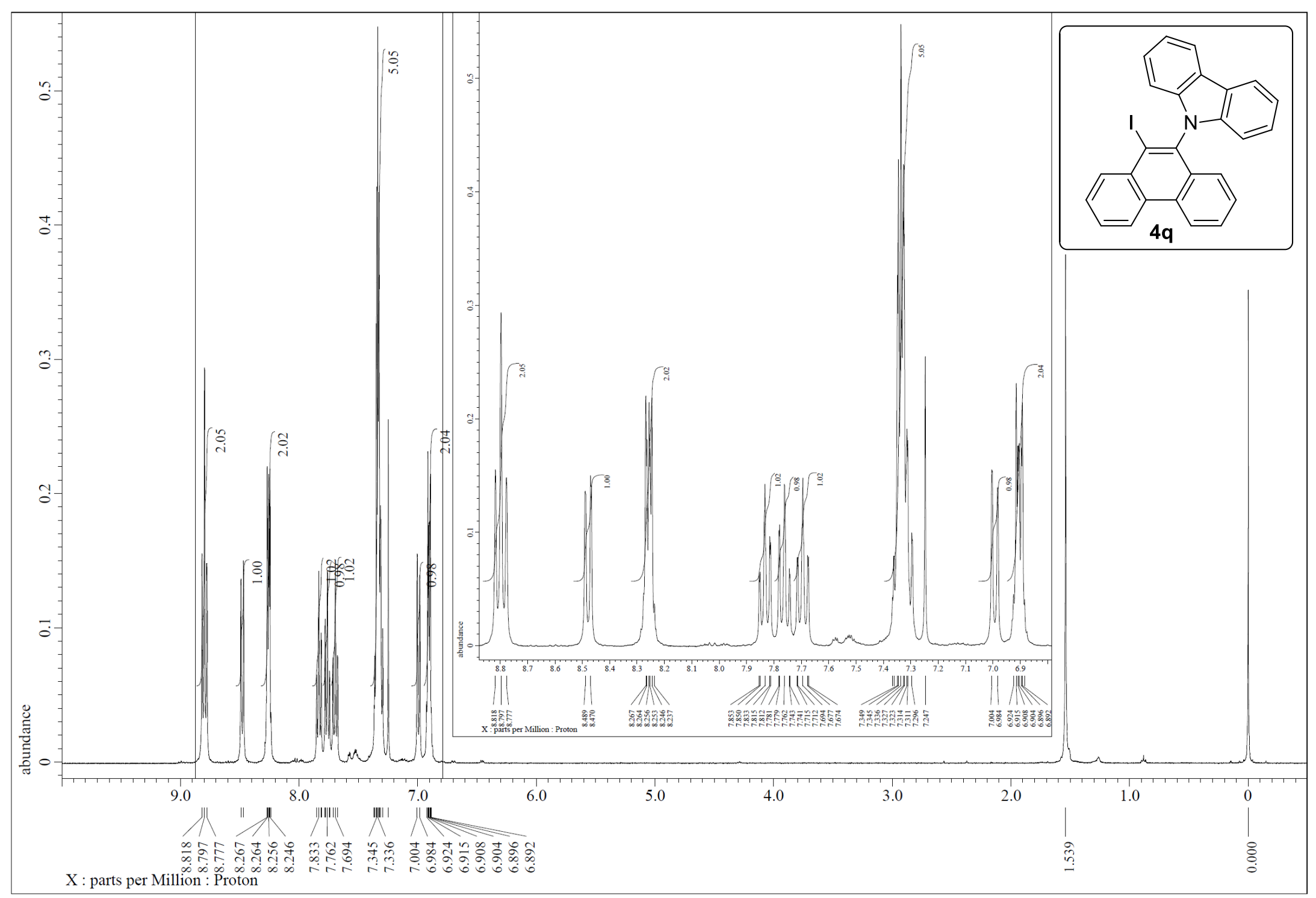

Figure S79. ${ }^{1} \mathrm{H}$ NMR (400 MHz) spectrum of $\mathbf{4 q}\left(\mathrm{CDCl}_{3}, \mathrm{rt}\right)$ 


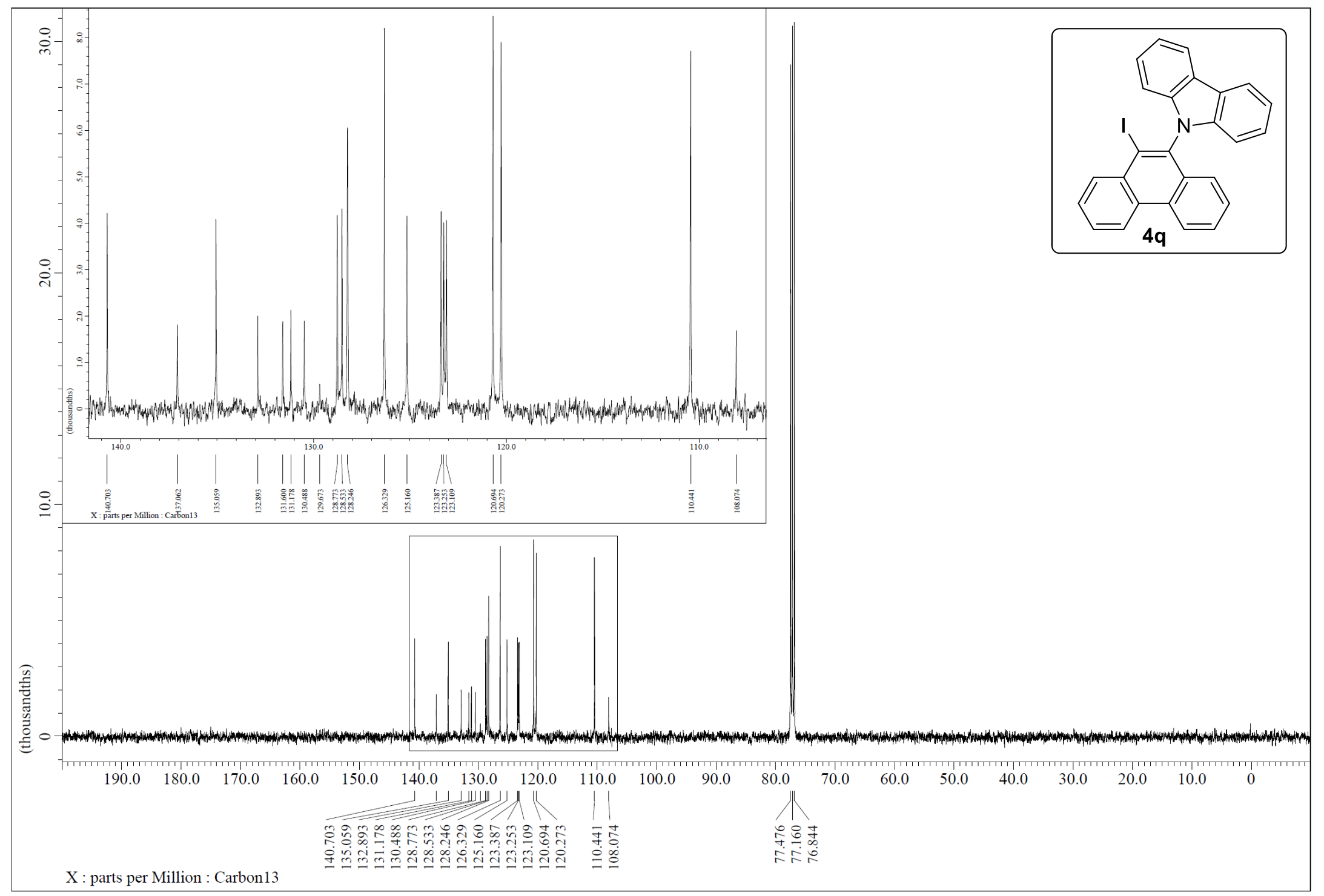

Figure S80. ${ }^{13} \mathrm{C}\left\{{ }^{1} \mathrm{H}\right\}$ NMR (101 MHz) spectrum of $\mathbf{4 q}\left(\mathrm{CDCl}_{3}, \mathrm{rt}\right)$ 


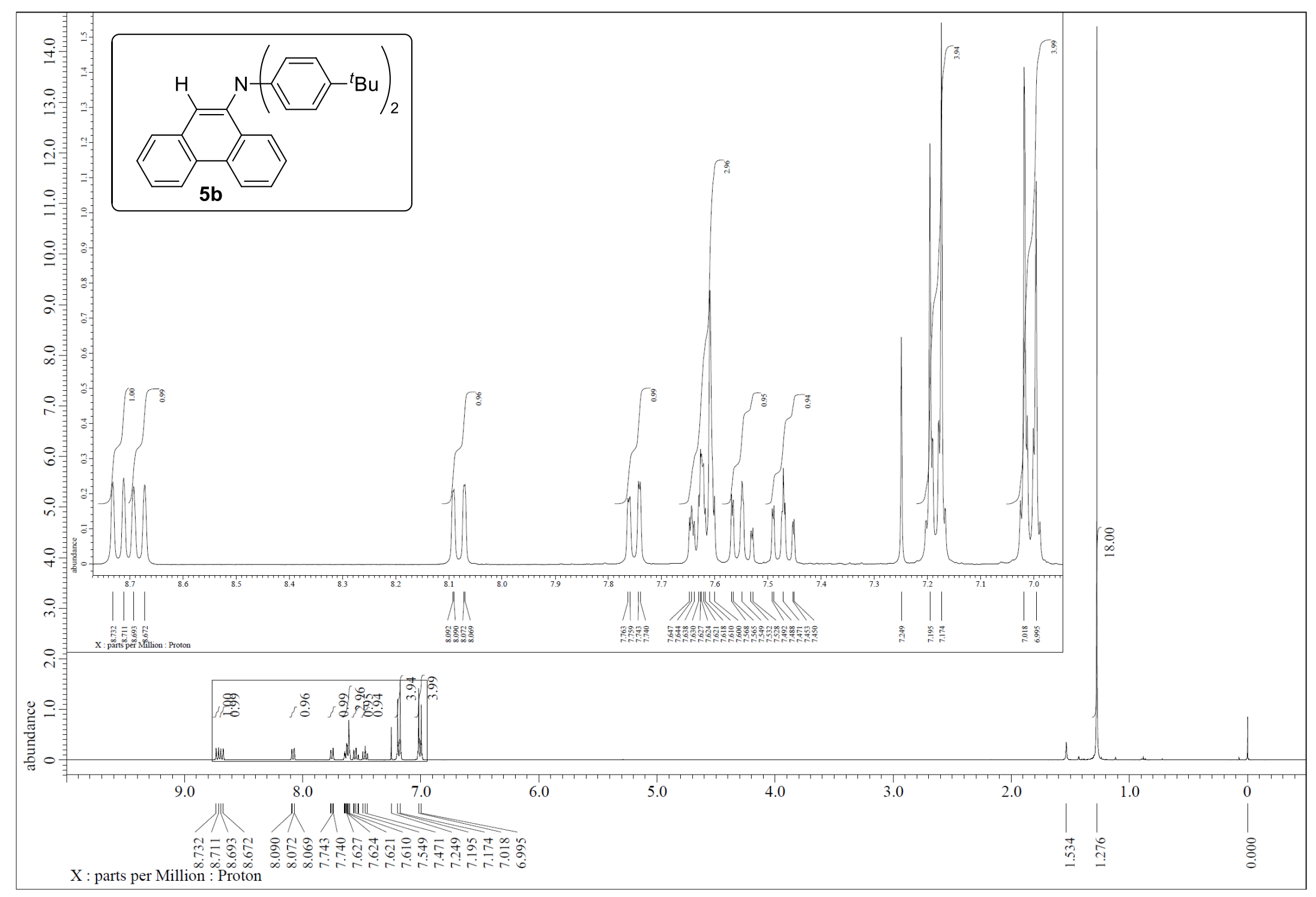

Figure S81. ${ }^{1} \mathrm{H}$ NMR (400 MHz) spectrum of $\mathbf{5 b}\left(\mathrm{CDCl}_{3}, \mathrm{rt}\right)$ 


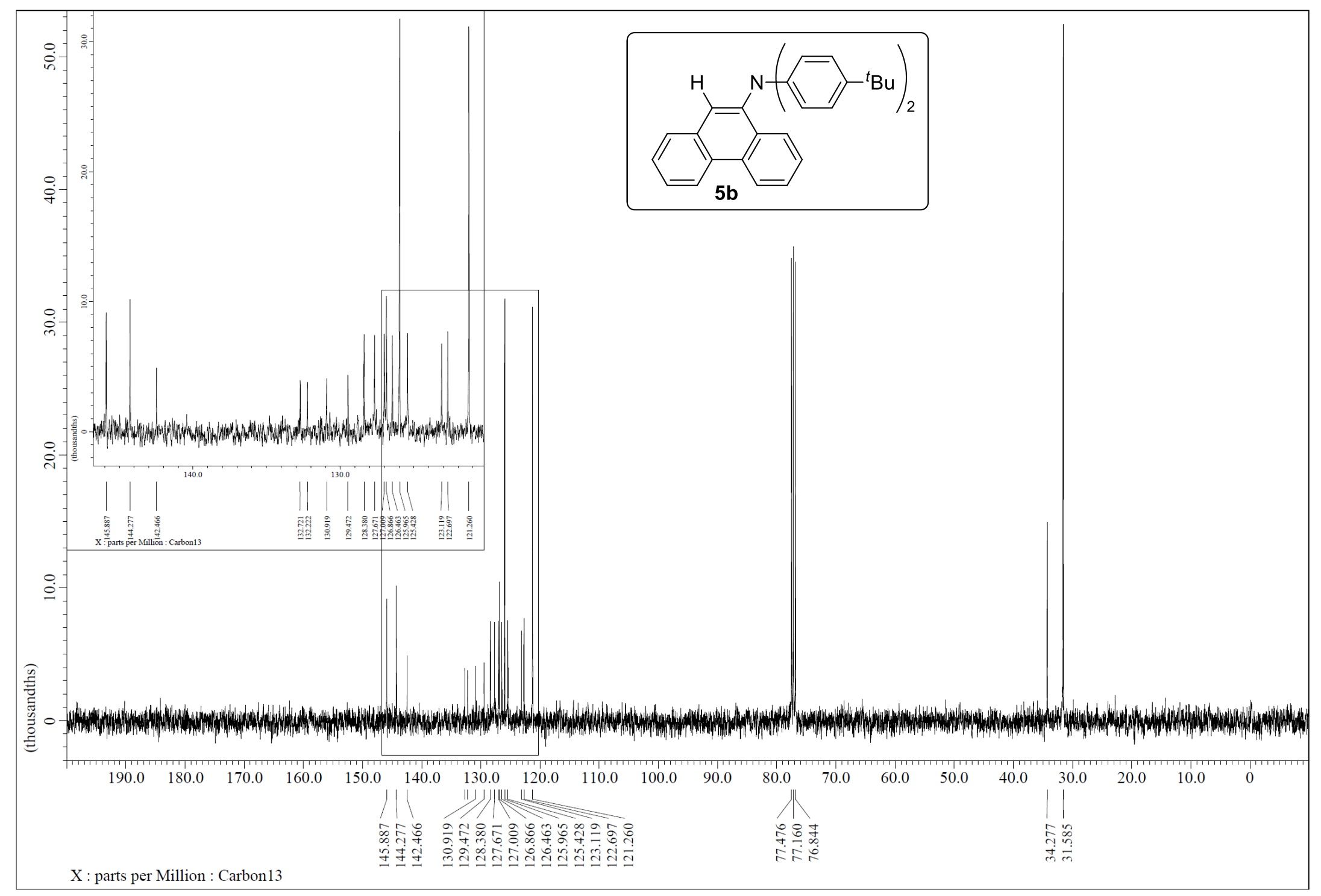

Figure S82. ${ }^{13} \mathrm{C}\left\{{ }^{1} \mathrm{H}\right\} \mathrm{NMR}(101 \mathrm{MHz})$ spectrum of $\mathbf{5 b}\left(\mathrm{CDCl}_{3}, \mathrm{rt}\right)$ 


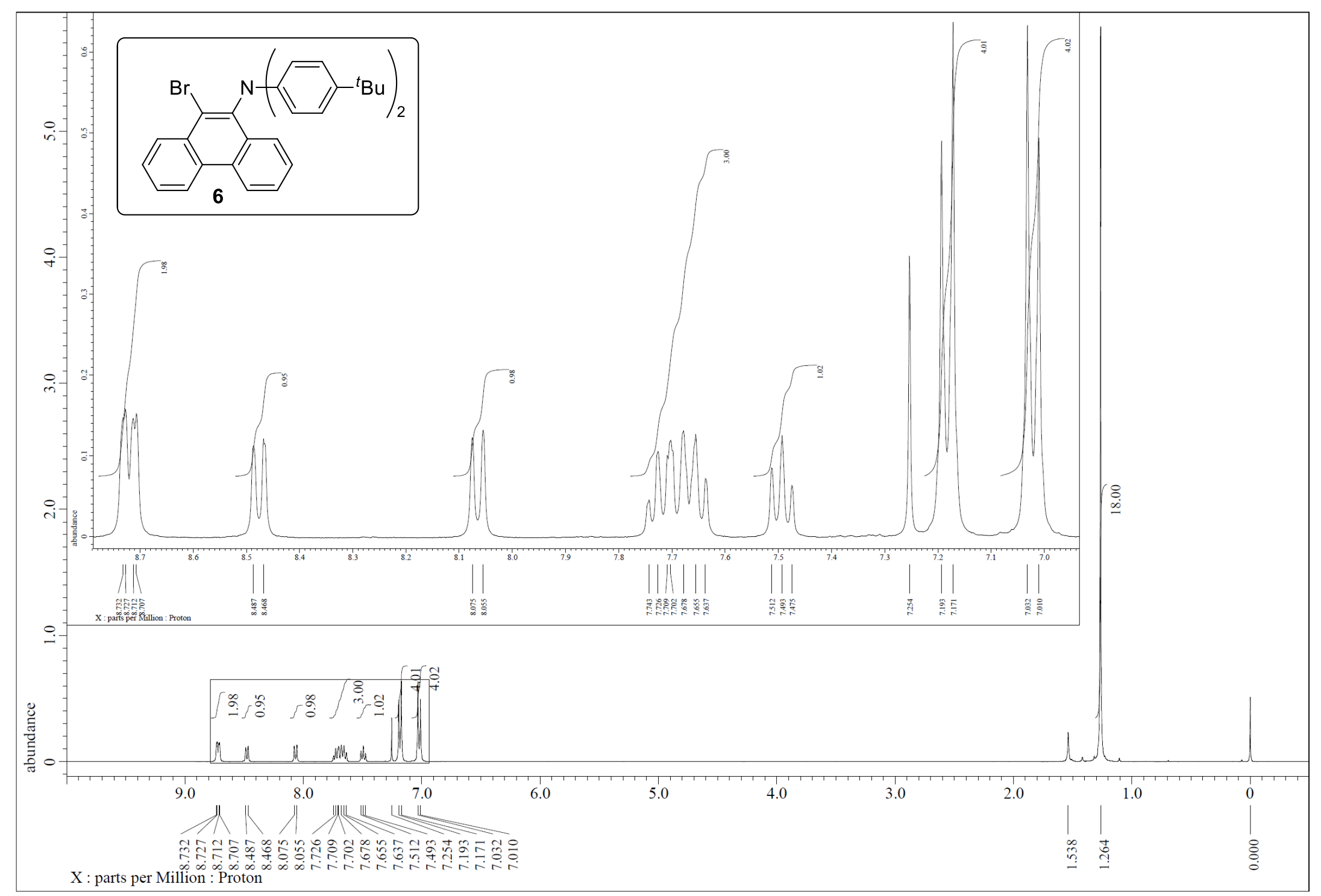

Figure S83. ${ }^{1} \mathrm{H}$ NMR $(400 \mathrm{MHz})$ spectrum of $\mathbf{6}\left(\mathrm{CDCl}_{3}, \mathrm{rt}\right)$ 


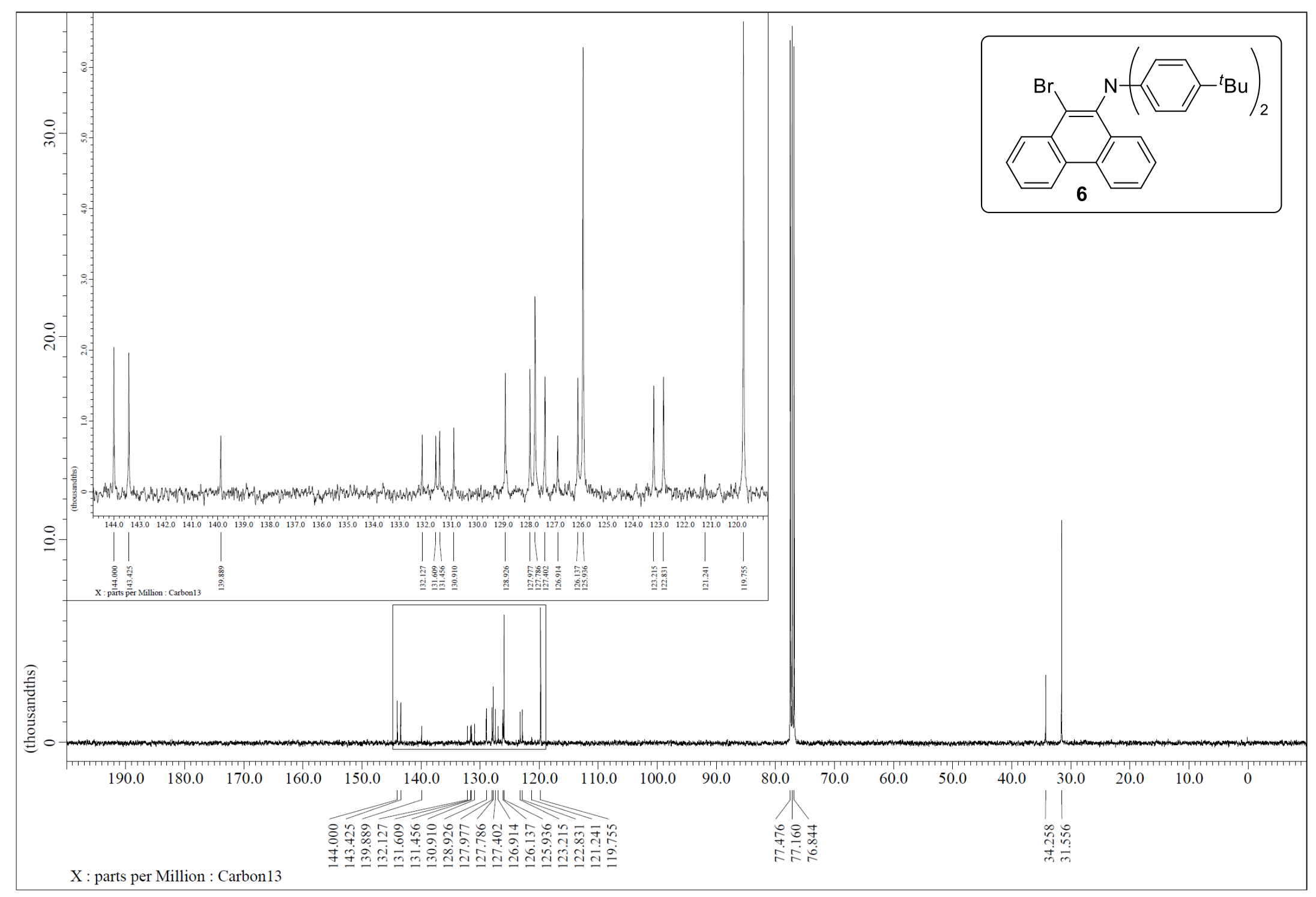

Figure S84. ${ }^{13} \mathrm{C}\left\{{ }^{1} \mathrm{H}\right\}$ NMR $(101 \mathrm{MHz})$ spectrum of $\mathbf{6}\left(\mathrm{CDCl}_{3}\right.$, rt) 


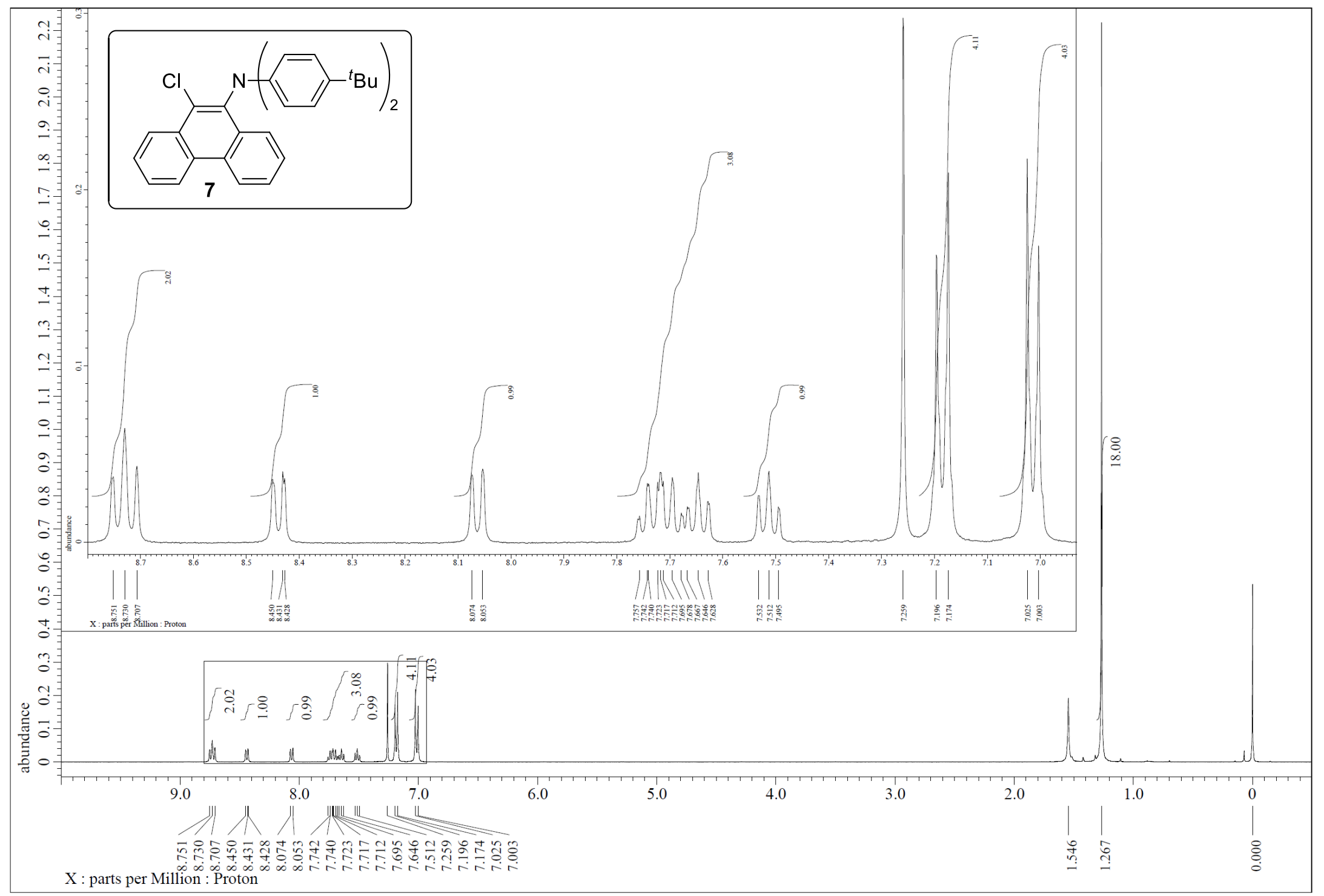

Figure S85. ${ }^{1} \mathrm{H}$ NMR $(400 \mathrm{MHz})$ spectrum of $7\left(\mathrm{CDCl}_{3}, \mathrm{rt}\right)$ 


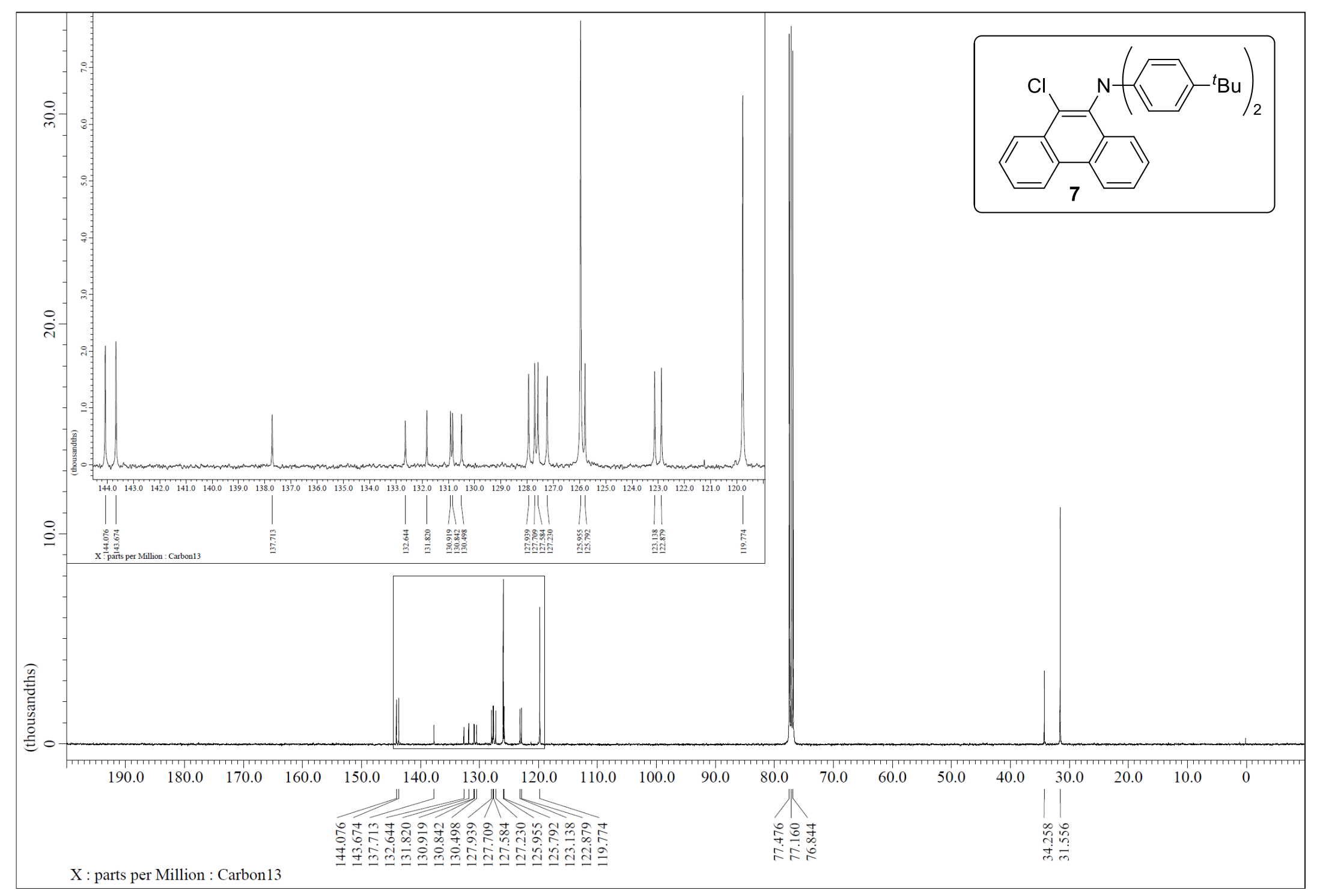

Figure S86. ${ }^{13} \mathrm{C}\left\{{ }^{1} \mathrm{H}\right\}$ NMR $(101 \mathrm{MHz})$ spectrum of $7\left(\mathrm{CDCl}_{3}, \mathrm{rt}\right)$ 


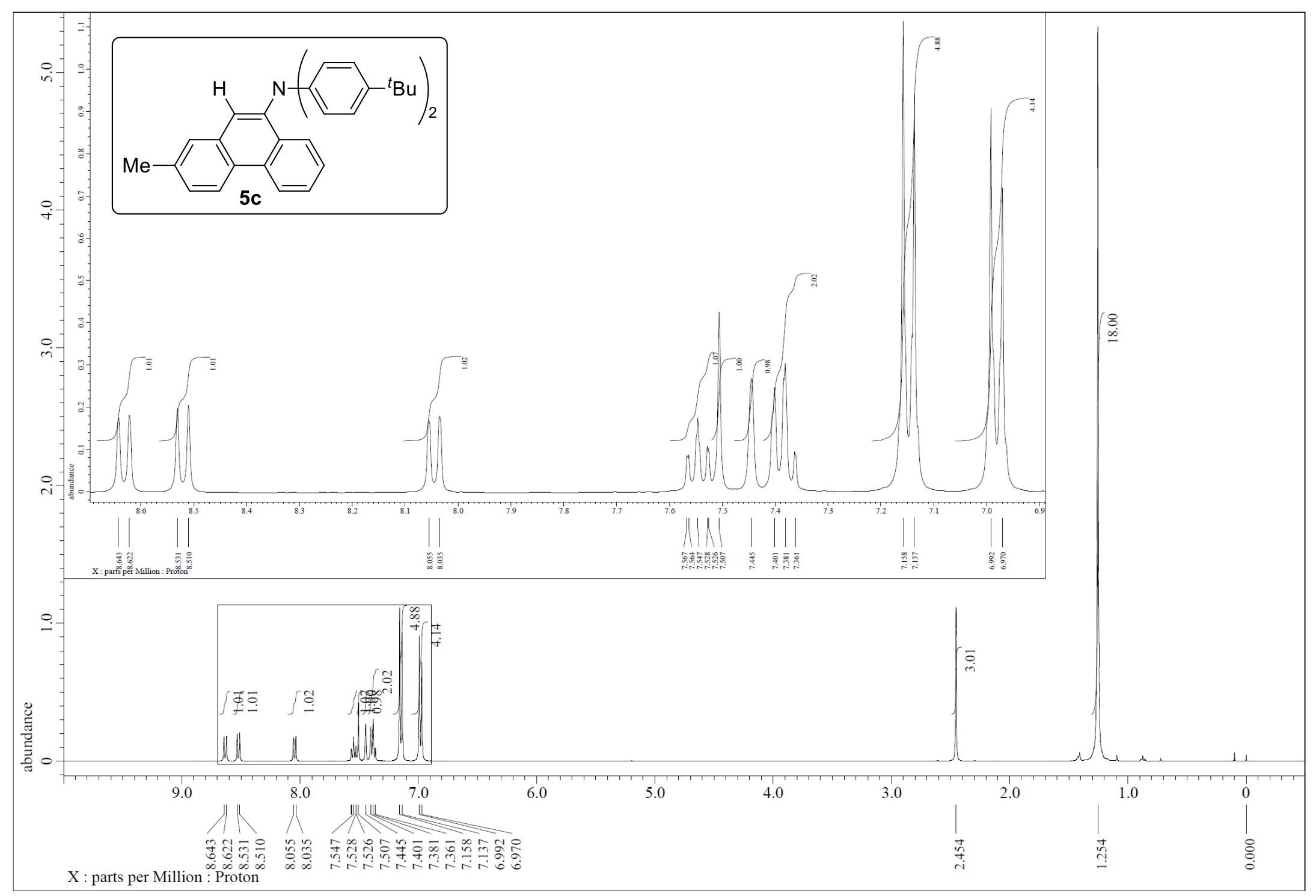

Figure S87. ${ }^{1} \mathrm{H}$ NMR $(400 \mathrm{MHz})$ spectrum of $\mathbf{5 c}\left(\mathrm{CDCl}_{3}, \mathrm{rt}\right)$ 


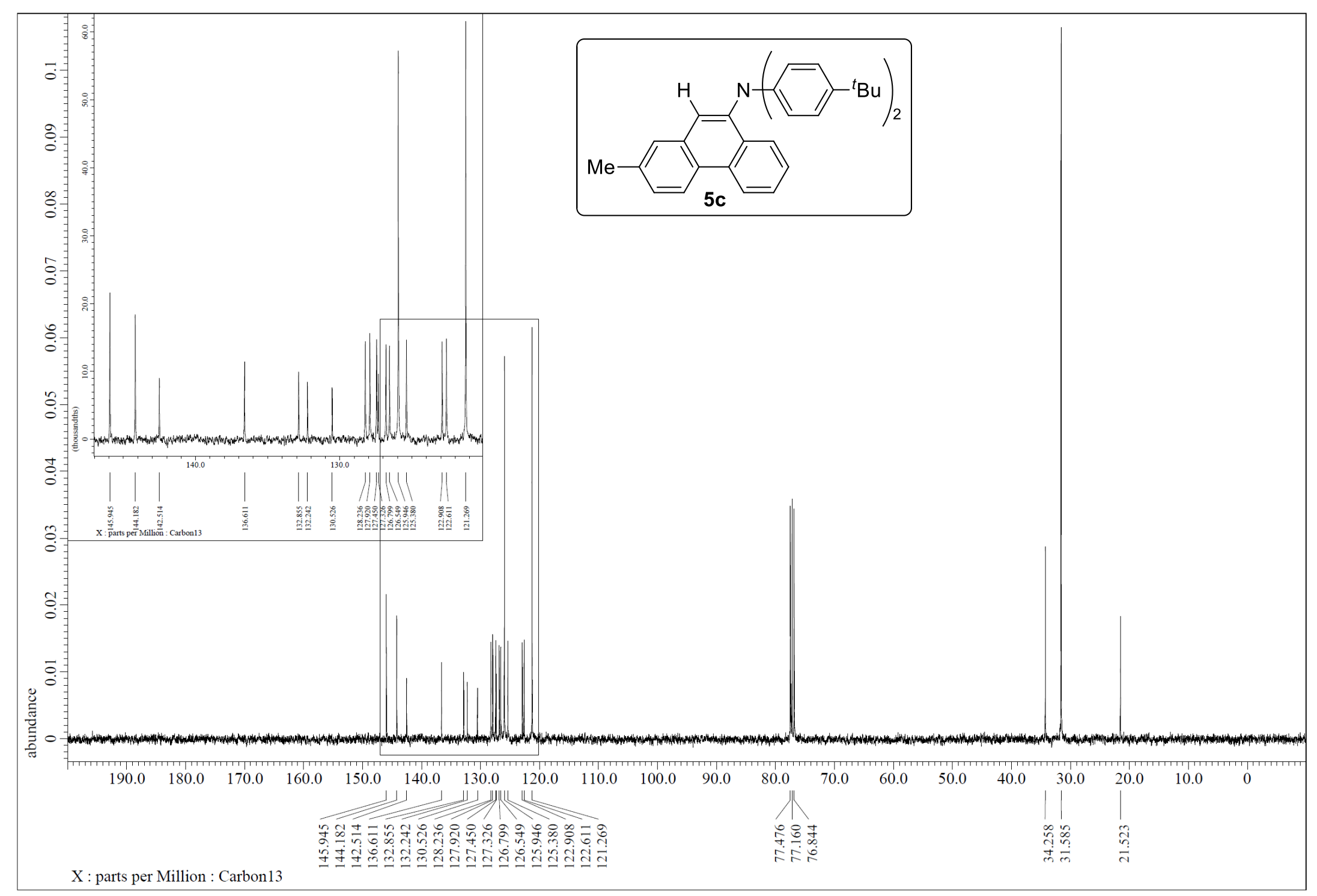

Figure S88. ${ }^{13} \mathrm{C}\left\{{ }^{1} \mathrm{H}\right\}$ NMR $(101 \mathrm{MHz})$ spectrum of $\mathbf{5 c}\left(\mathrm{CDCl}_{3}, \mathrm{rt}\right)$ 


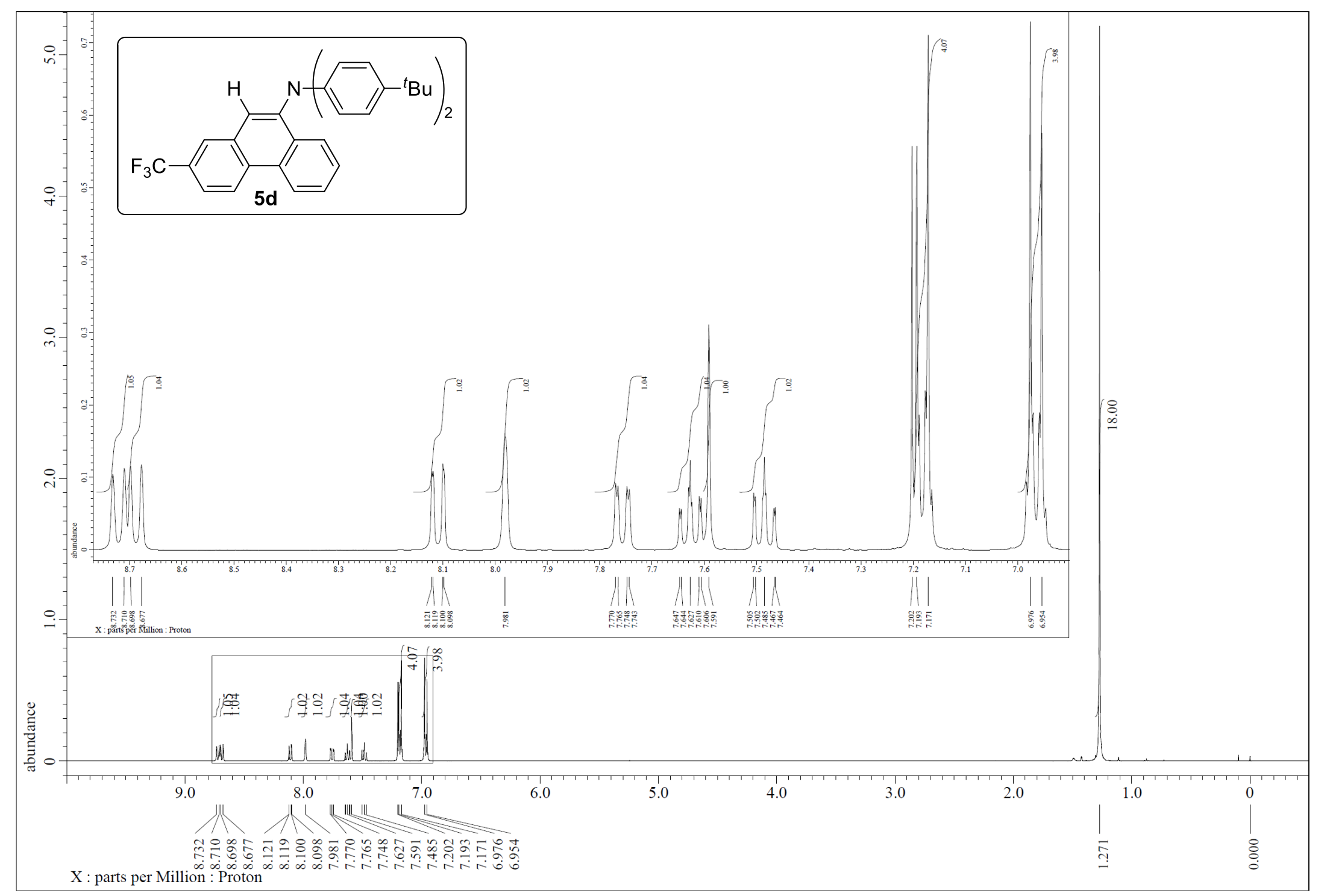

Figure S89. ${ }^{1} \mathrm{H}$ NMR (400 MHz) spectrum of $\mathbf{5 d}\left(\mathrm{CDCl}_{3}, \mathrm{rt}\right)$ 


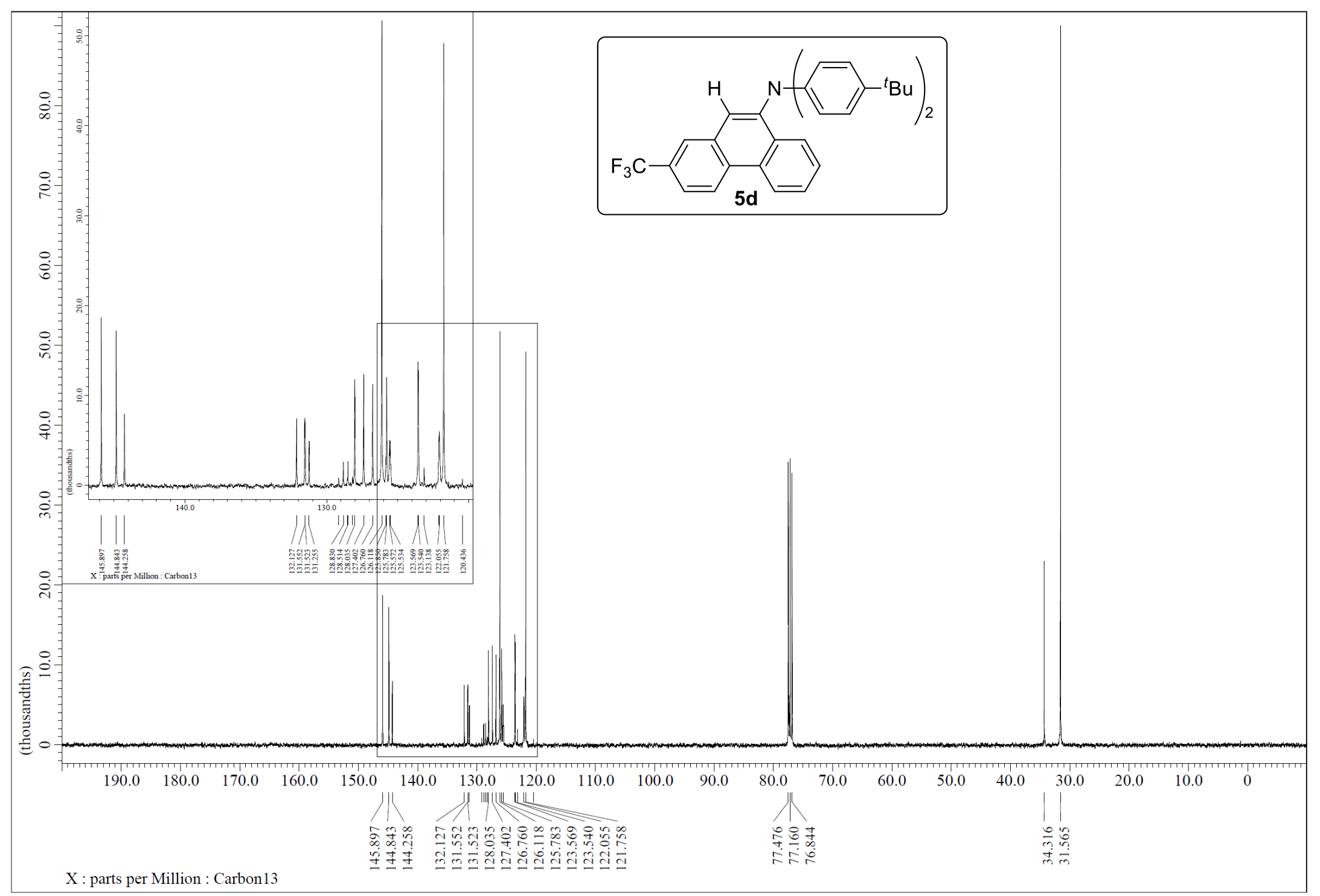

Figure S90. ${ }^{13} \mathrm{C}\left\{{ }^{1} \mathrm{H}\right\}$ NMR $(101 \mathrm{MHz})$ spectrum of $\mathbf{5 d}\left(\mathrm{CDCl}_{3}, \mathrm{rt}\right)$ 


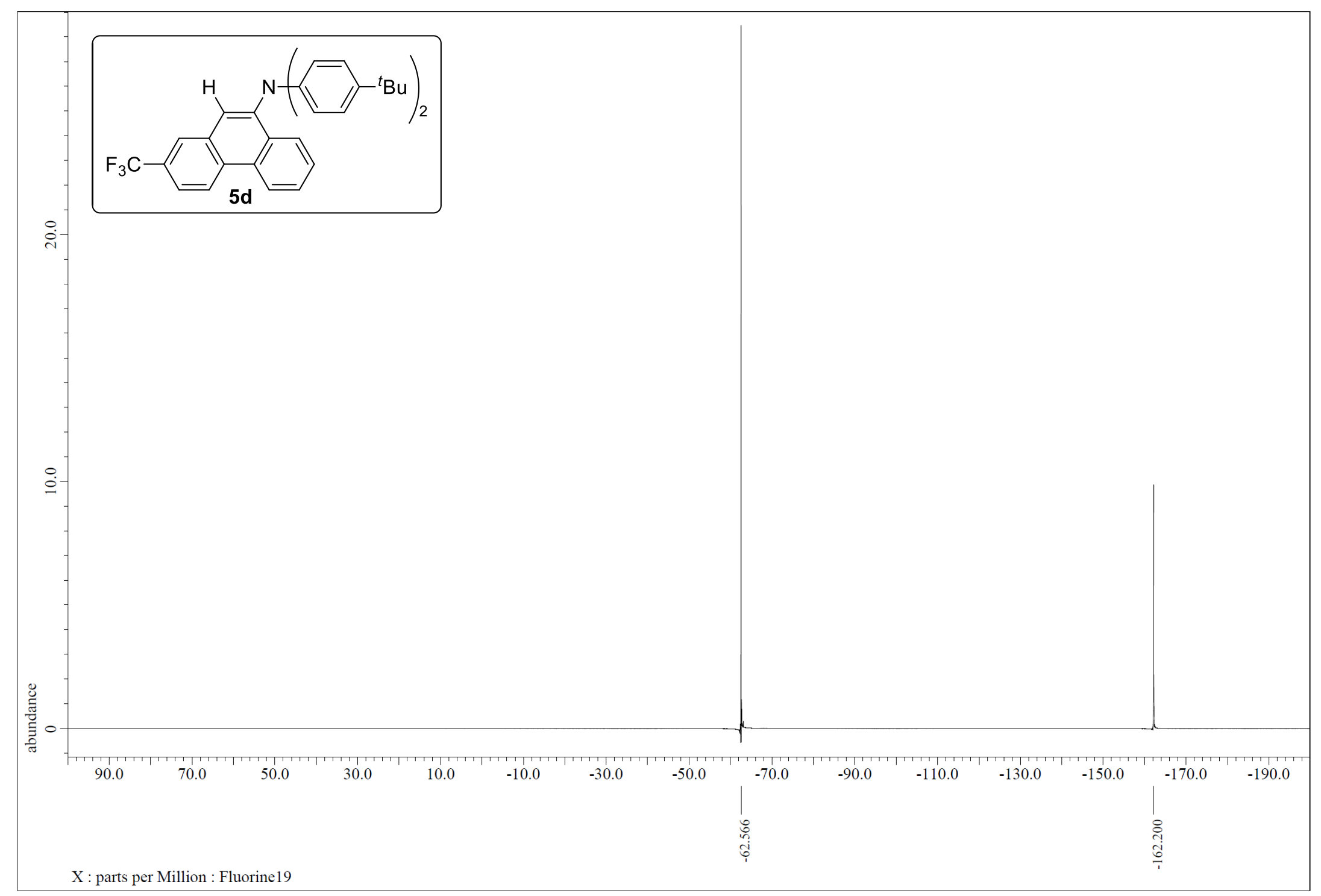

Figure S91. ${ }^{19} \mathrm{~F}\left\{{ }^{1} \mathrm{H}\right\}$ NMR $(376 \mathrm{MHz})$ spectrum of $\mathbf{5 d}\left(\mathrm{CDCl}_{3}, \mathrm{rt}\right)$ 


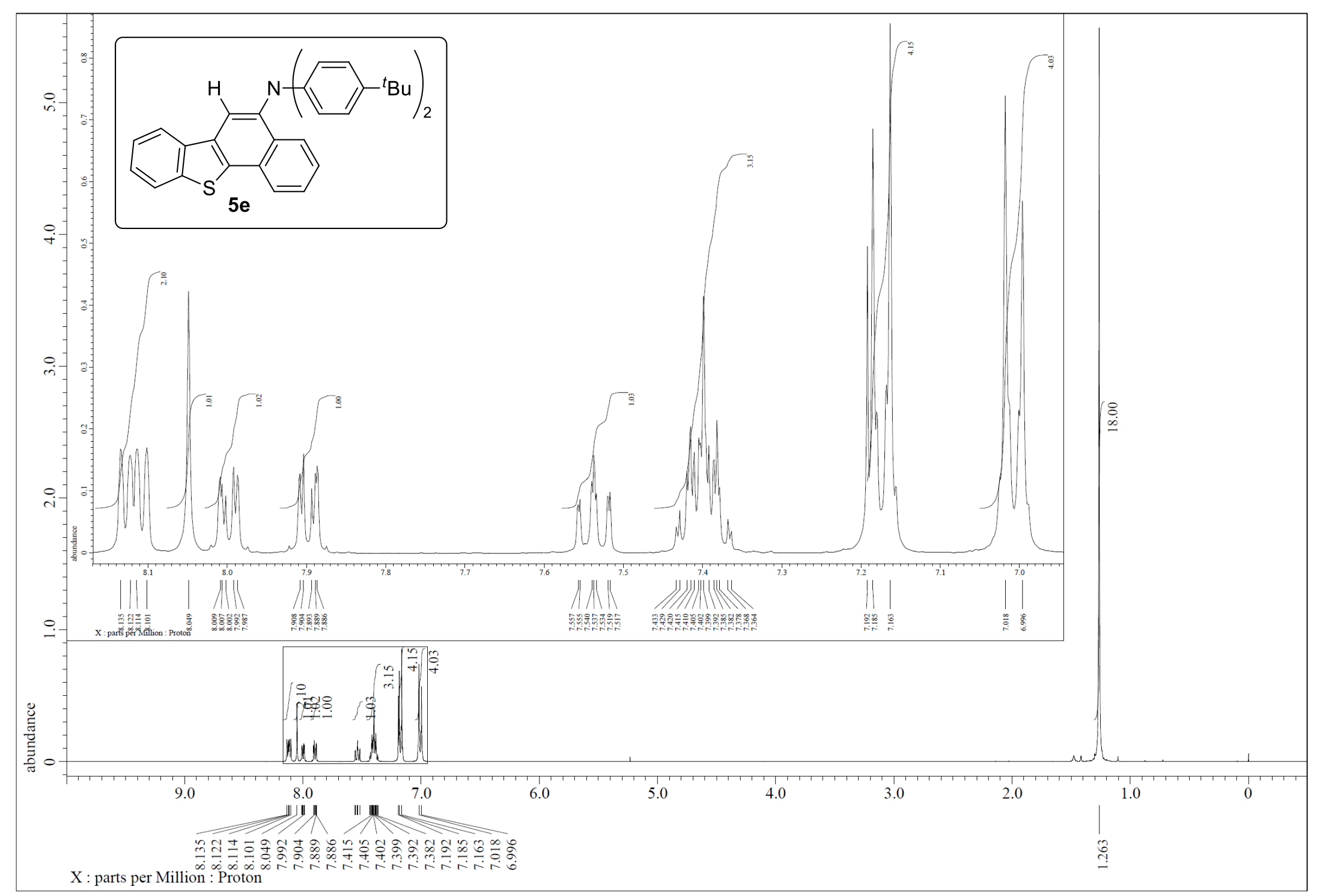

Figure S92. ${ }^{1} \mathrm{H}$ NMR $(400 \mathrm{MHz})$ spectrum of $\mathbf{5 e}\left(\mathrm{CDCl}_{3}, \mathrm{rt}\right)$ 


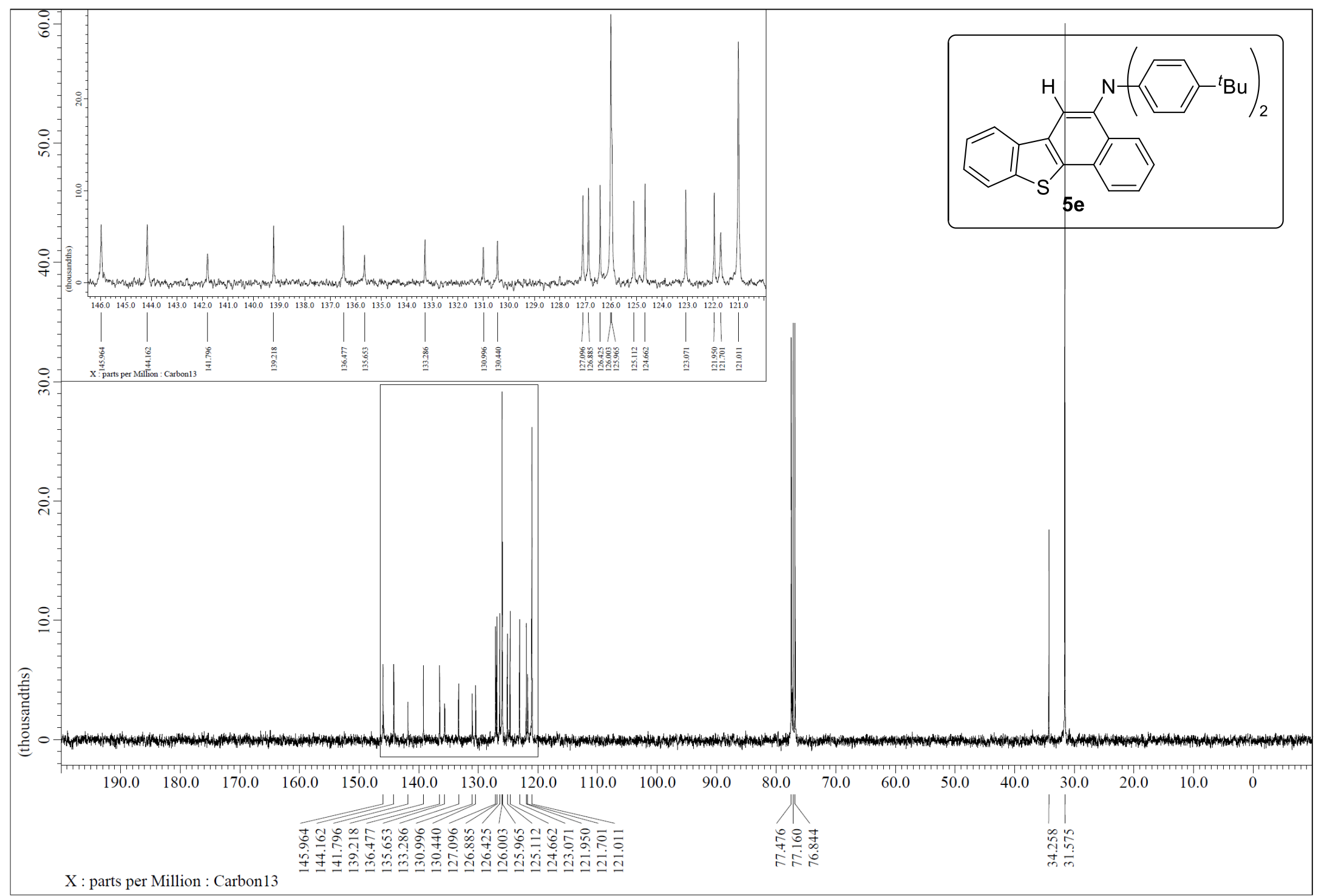

Figure S93. ${ }^{13} \mathrm{C}\left\{{ }^{1} \mathrm{H}\right\}$ NMR $(101 \mathrm{MHz})$ spectrum of $\mathbf{5 e}\left(\mathrm{CDCl}_{3}, \mathrm{rt}\right)$ 


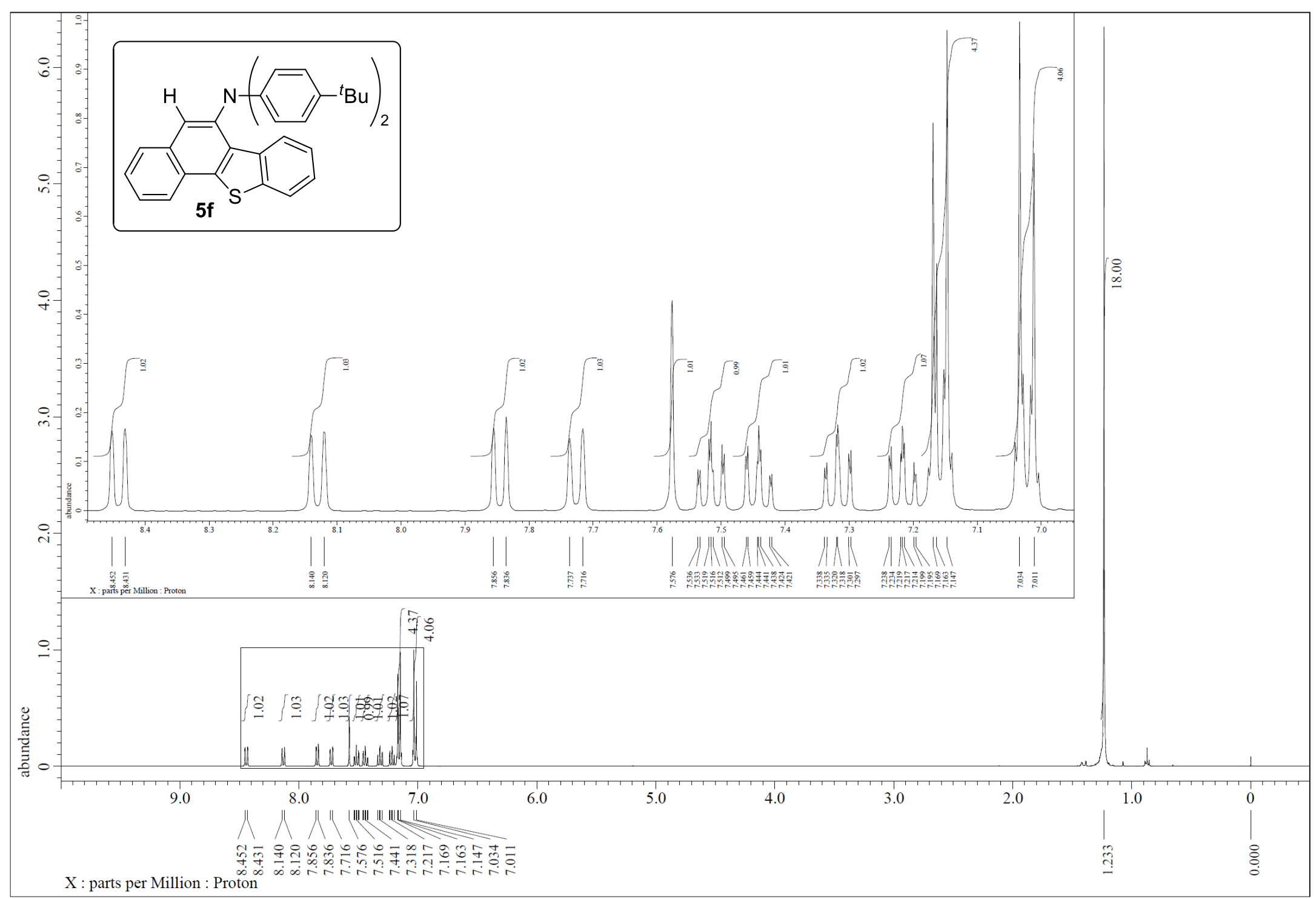

Figure S94. ${ }^{1} \mathrm{H}$ NMR $(400 \mathrm{MHz})$ spectrum of $\mathbf{5 f}\left(\mathrm{CDCl}_{3}, \mathrm{rt}\right)$ 


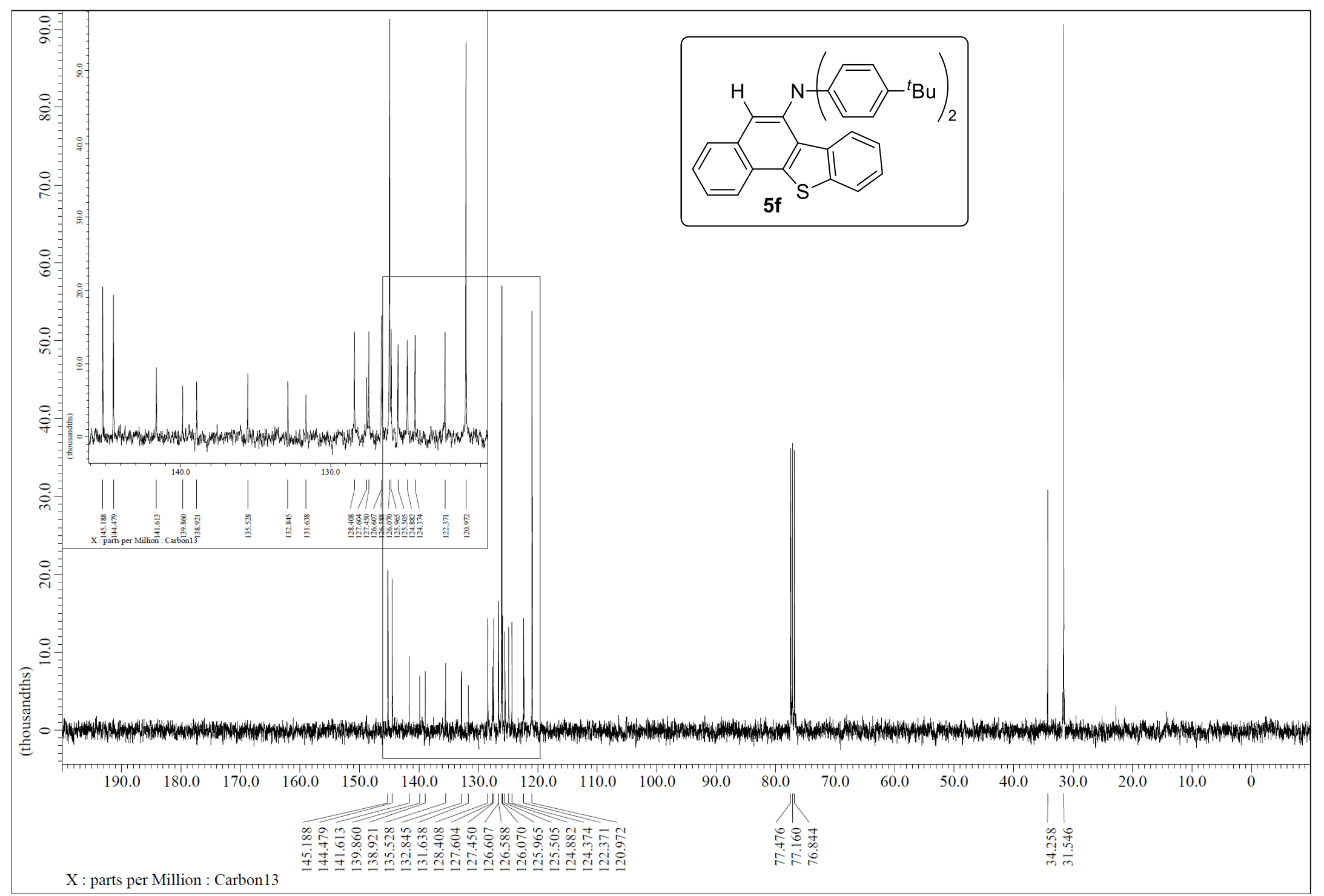

Figure S95. ${ }^{13} \mathrm{C}\left\{{ }^{1} \mathrm{H}\right\} \mathrm{NMR}(101 \mathrm{MHz})$ spectrum of $\mathbf{5 f}\left(\mathrm{CDCl}_{3}, \mathrm{rt}\right)$ 


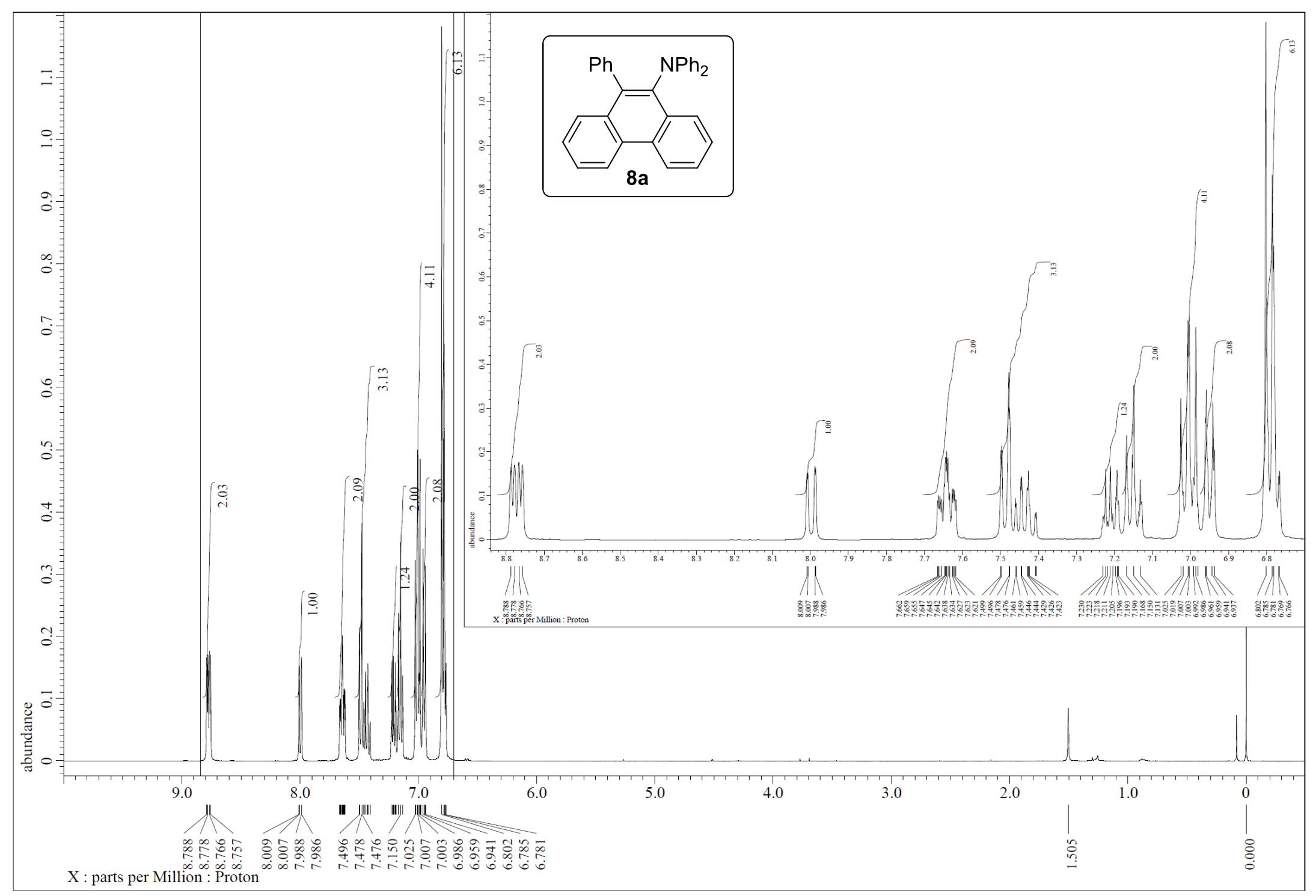

Figure S96. ${ }^{1} \mathrm{H}$ NMR $(400 \mathrm{MHz})$ spectrum of $\mathbf{8 a}\left(\mathrm{CDCl}_{3}, \mathrm{rt}\right)$ 


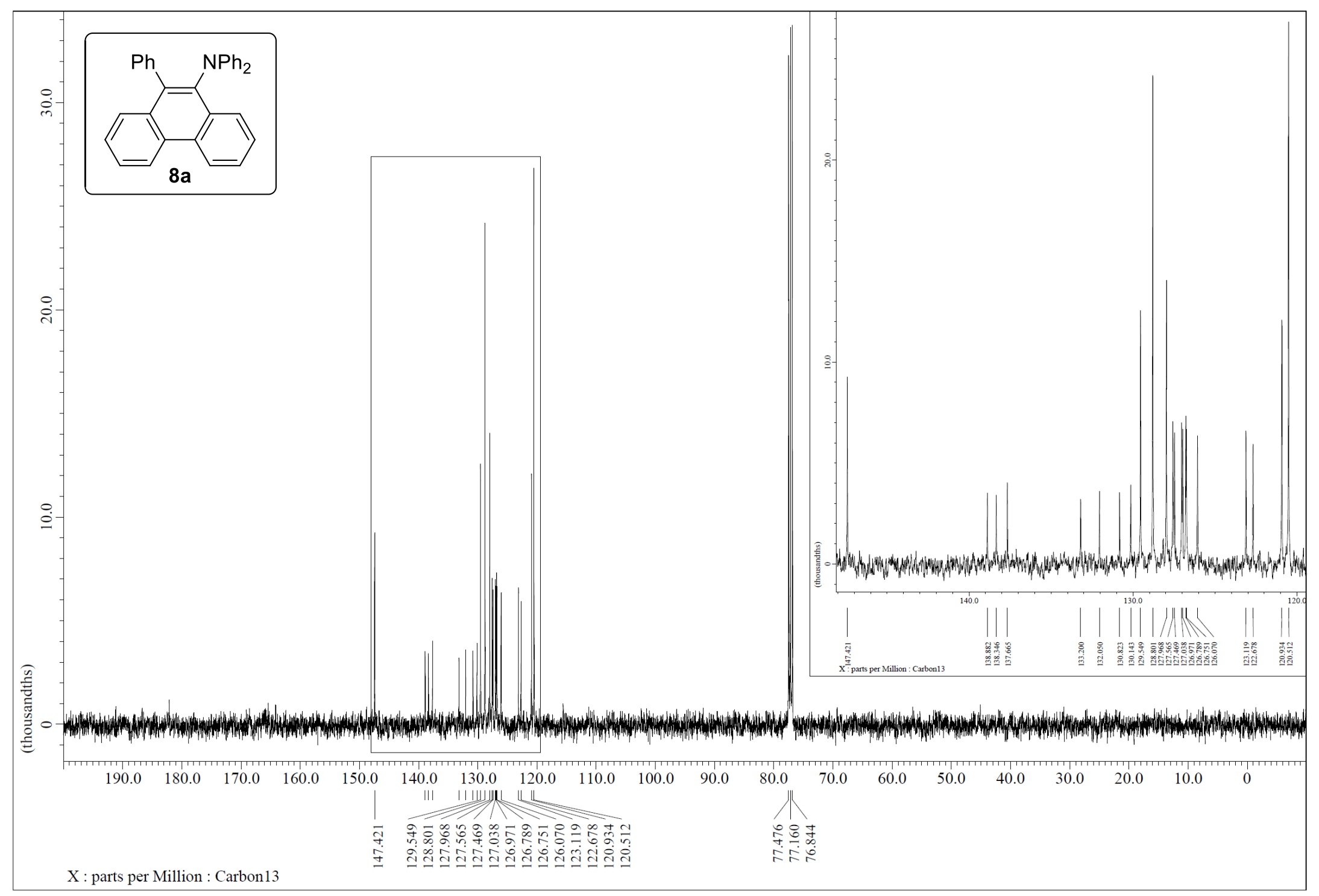

Figure S97. ${ }^{13} \mathrm{C}\left\{{ }^{1} \mathrm{H}\right\}$ NMR $(101 \mathrm{MHz})$ spectrum of $\mathbf{8 a}\left(\mathrm{CDCl}_{3}, \mathrm{rt}\right)$ 


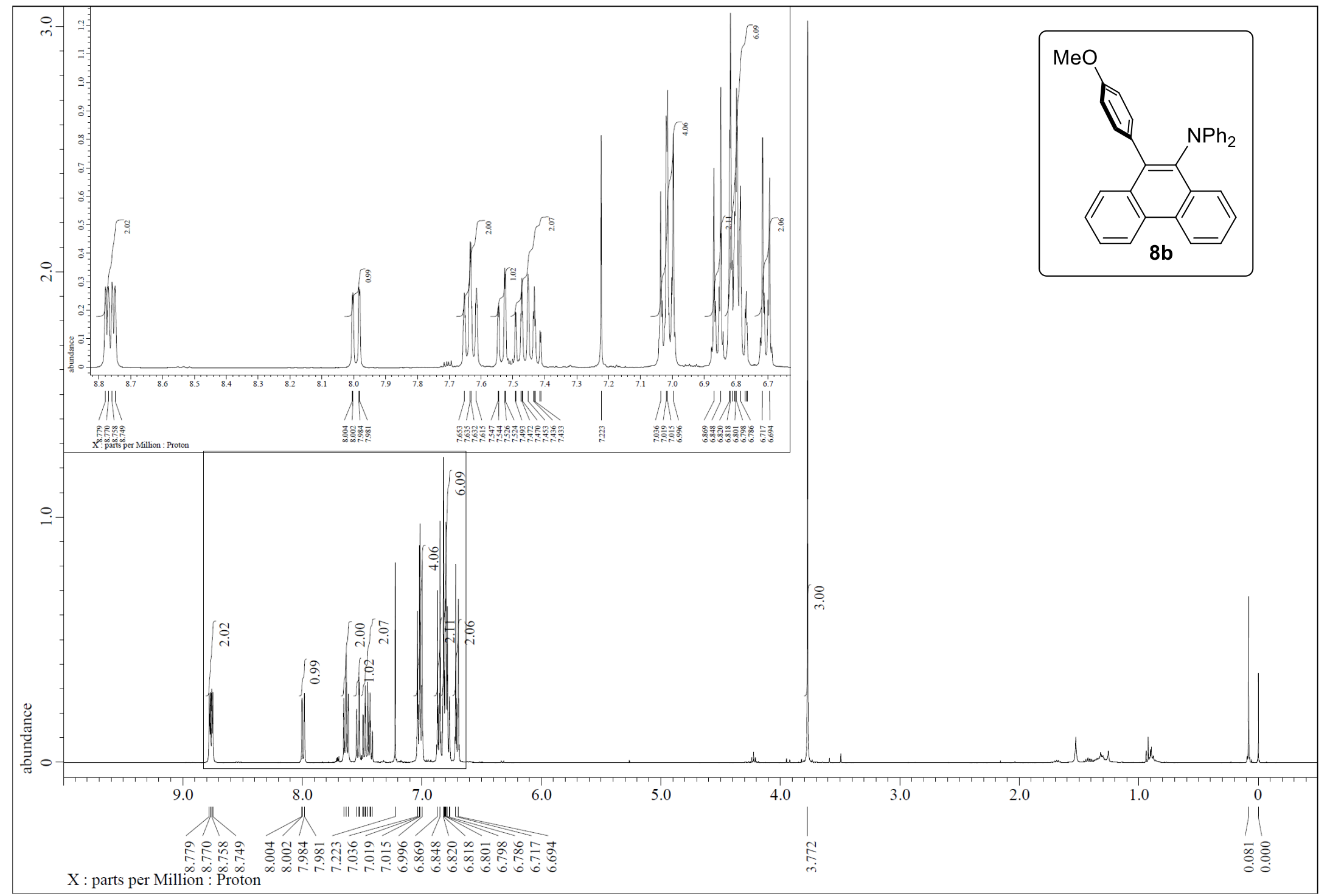

Figure S98. ${ }^{1} \mathrm{H}$ NMR (400 MHz) spectrum of $\mathbf{8 b}\left(\mathrm{CDCl}_{3}, \mathrm{rt}\right)$ 


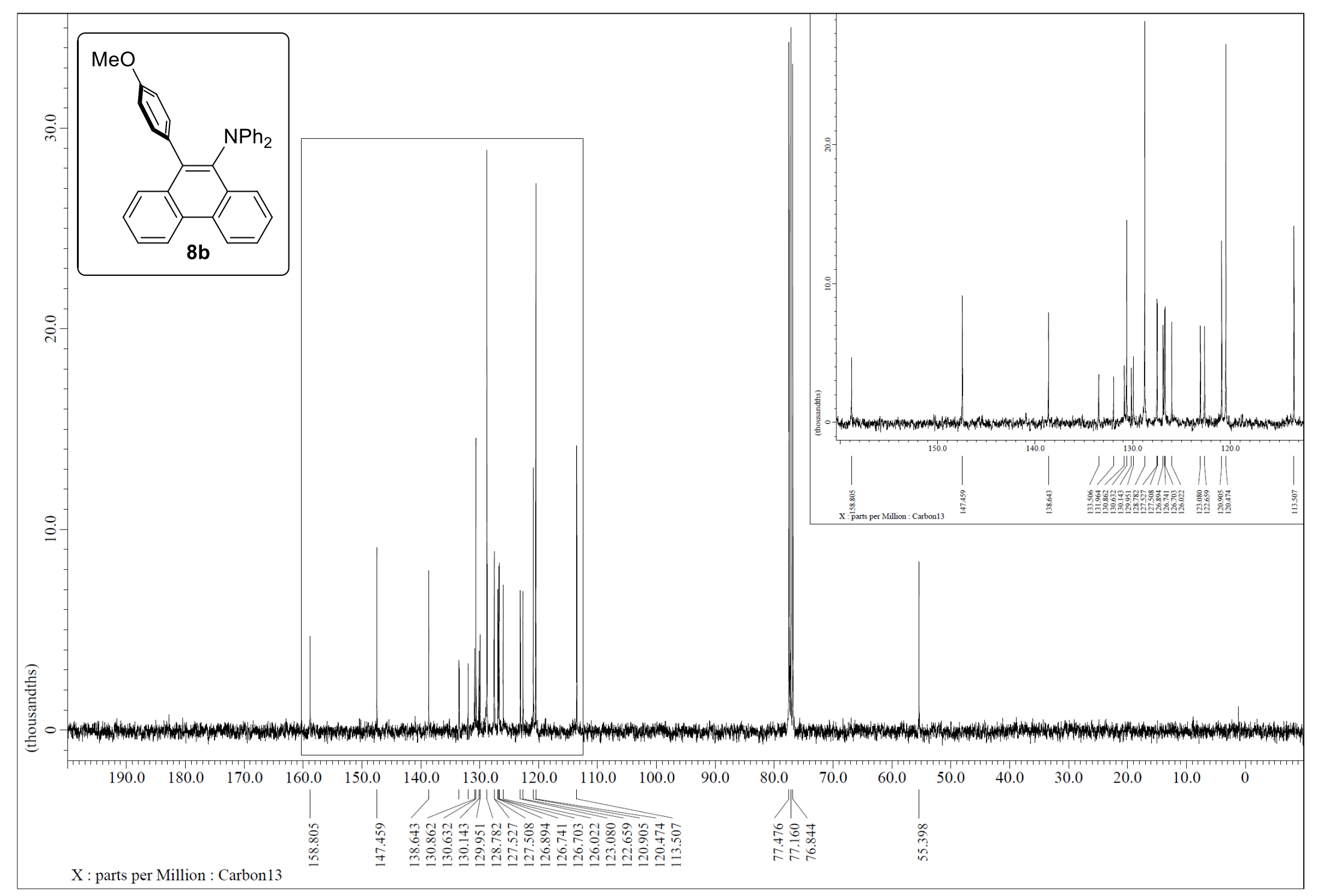

Figure S99. ${ }^{13} \mathrm{C}\left\{{ }^{1} \mathrm{H}\right\} \mathrm{NMR}(101 \mathrm{MHz})$ spectrum of $\mathbf{8 b}\left(\mathrm{CDCl}_{3}, \mathrm{rt}\right)$ 


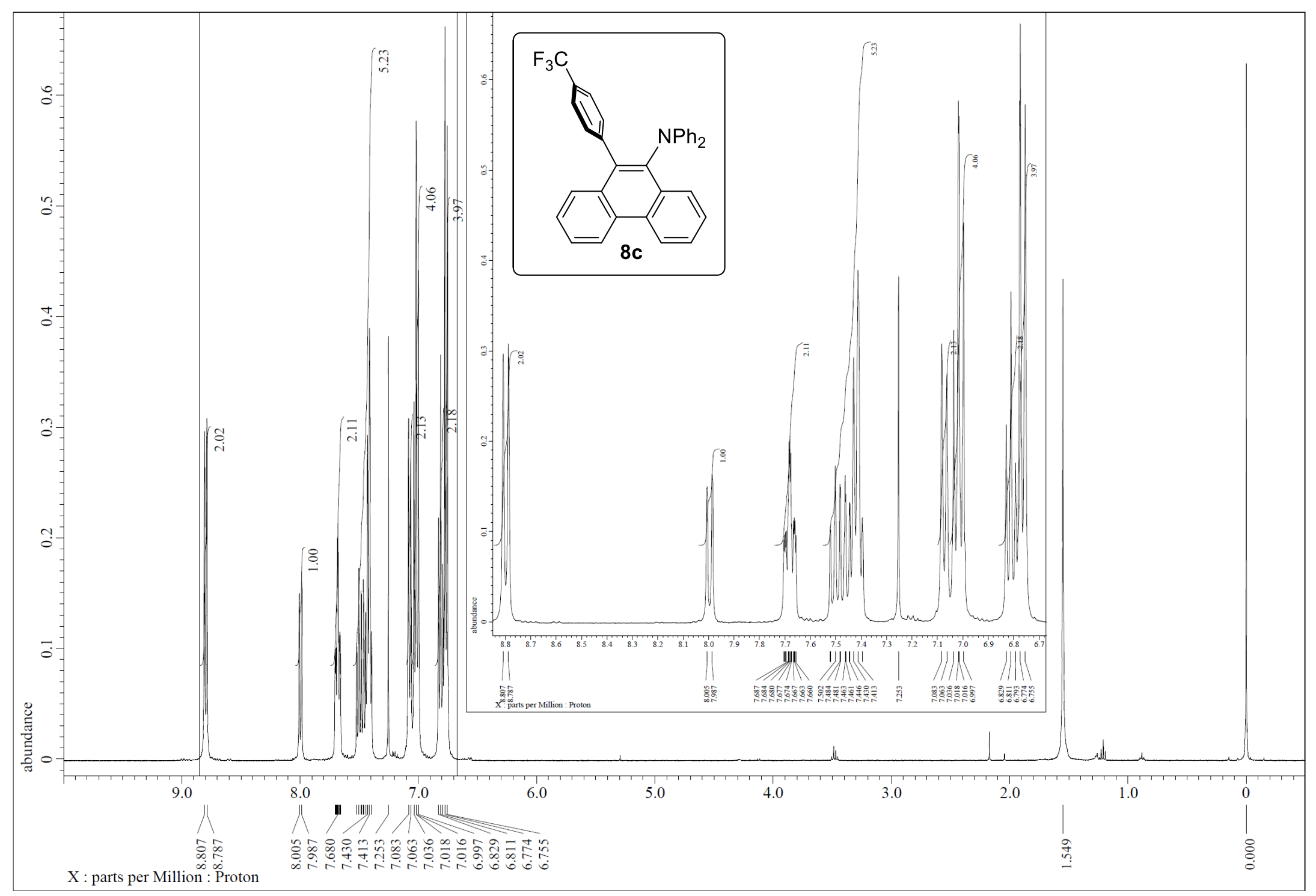

Figure S100. ${ }^{1} \mathrm{H}$ NMR $(400 \mathrm{MHz})$ spectrum of $\mathbf{8 c}\left(\mathrm{CDCl}_{3}, \mathrm{rt}\right)$ 


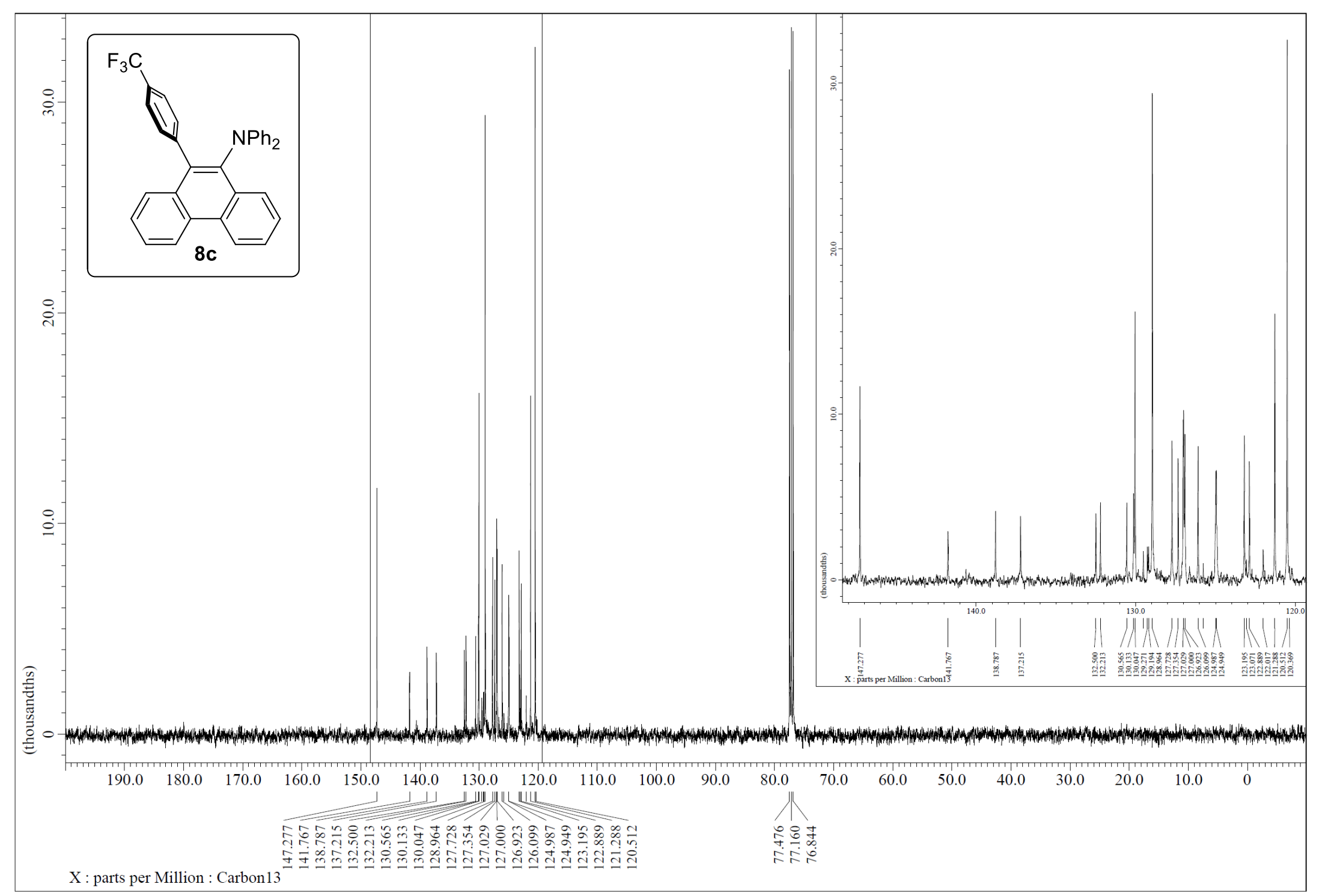

Figure S101. ${ }^{13} \mathrm{C}\left\{{ }^{1} \mathrm{H}\right\}$ NMR $(101 \mathrm{MHz})$ spectrum of $\mathbf{8 c}\left(\mathrm{CDCl}_{3}, \mathrm{rt}\right)$ 


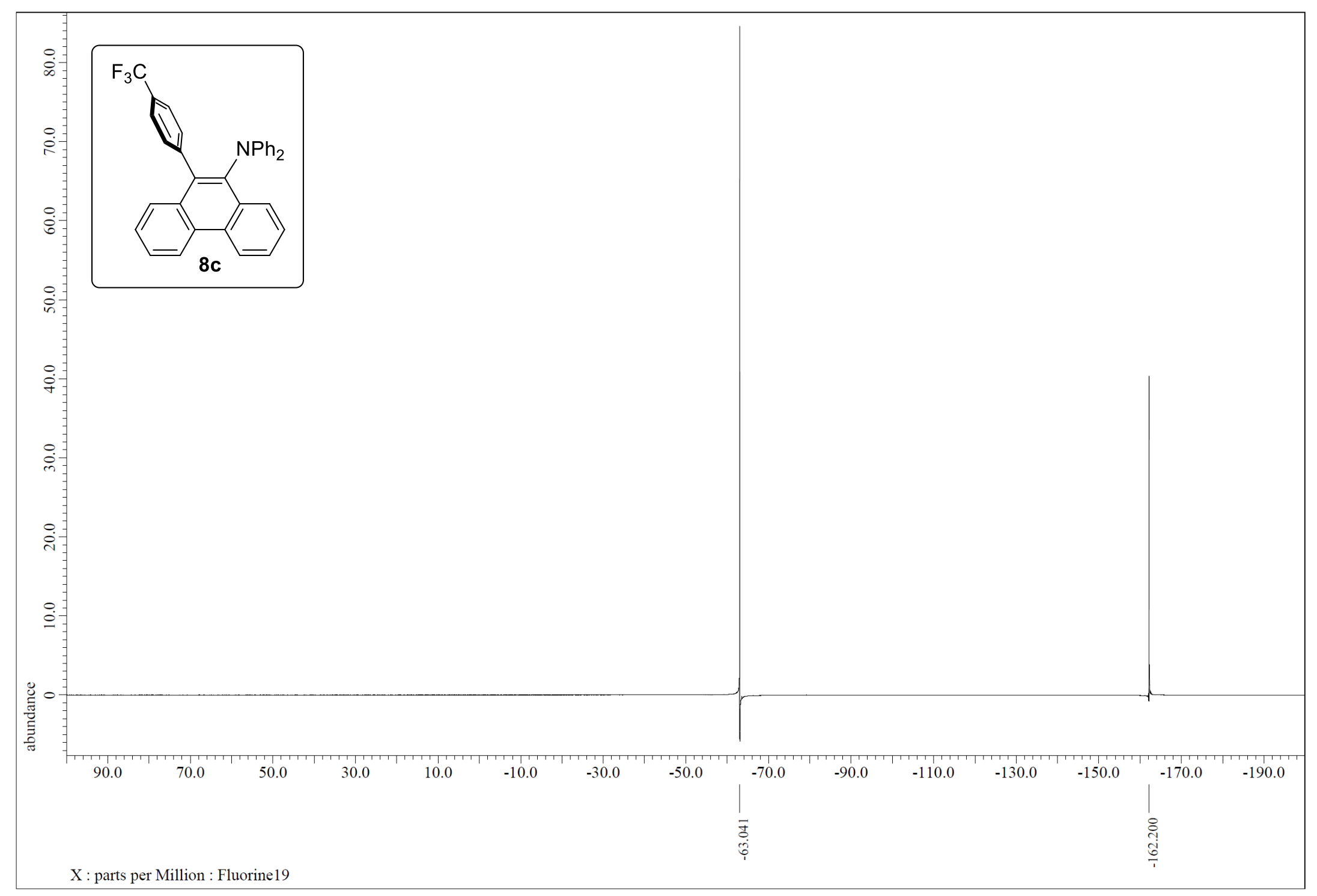

Figure S102. ${ }^{19} \mathrm{~F}\left\{{ }^{1} \mathrm{H}\right\}$ NMR $(376 \mathrm{MHz})$ spectrum of $\mathbf{8 c}\left(\mathrm{CDCl}_{3}, \mathrm{rt}\right)$ 


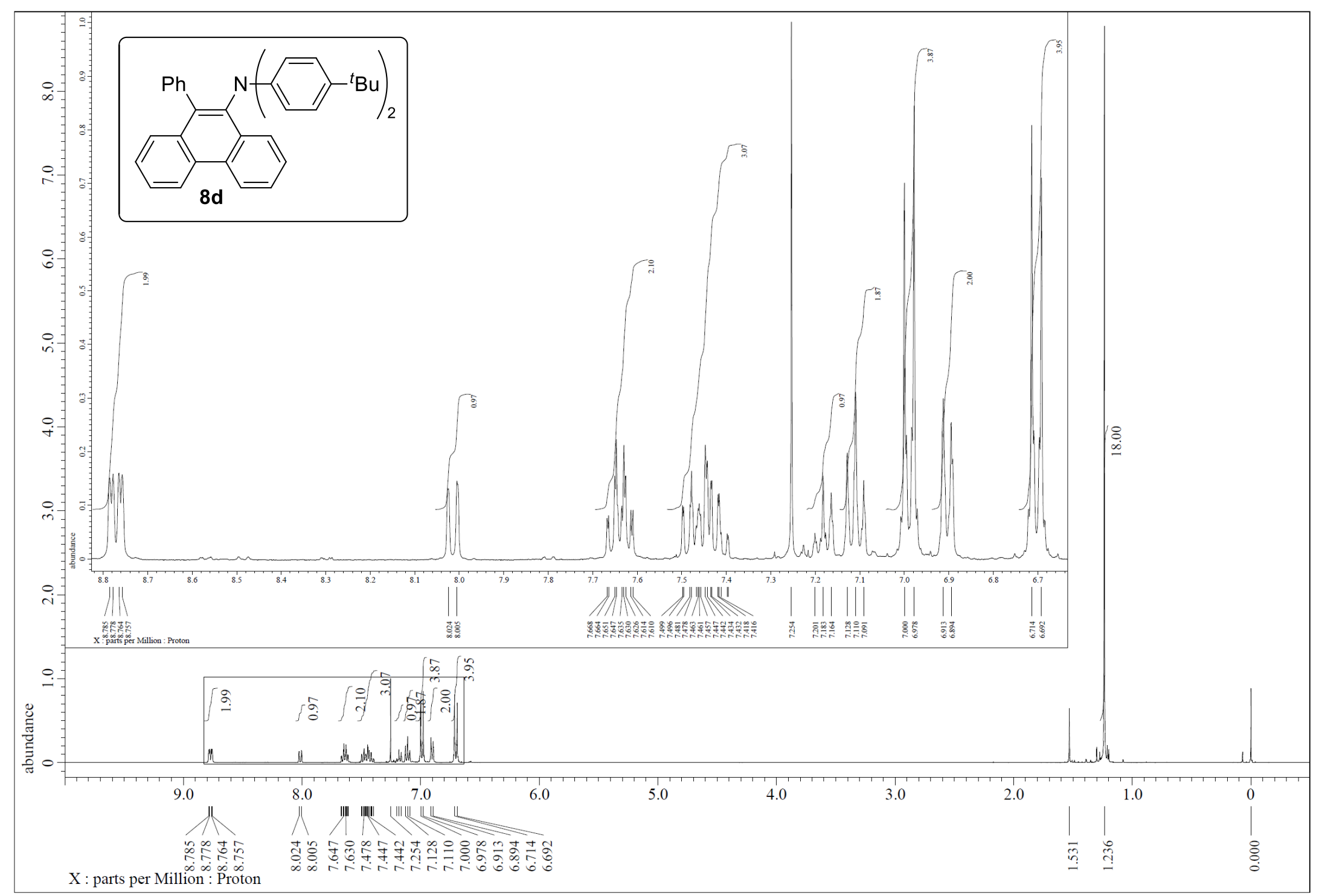

Figure S103. ${ }^{1} \mathrm{H}$ NMR (400 MHz) spectrum of $\mathbf{8 d}\left(\mathrm{CDCl}_{3}, \mathrm{rt}\right)$ 


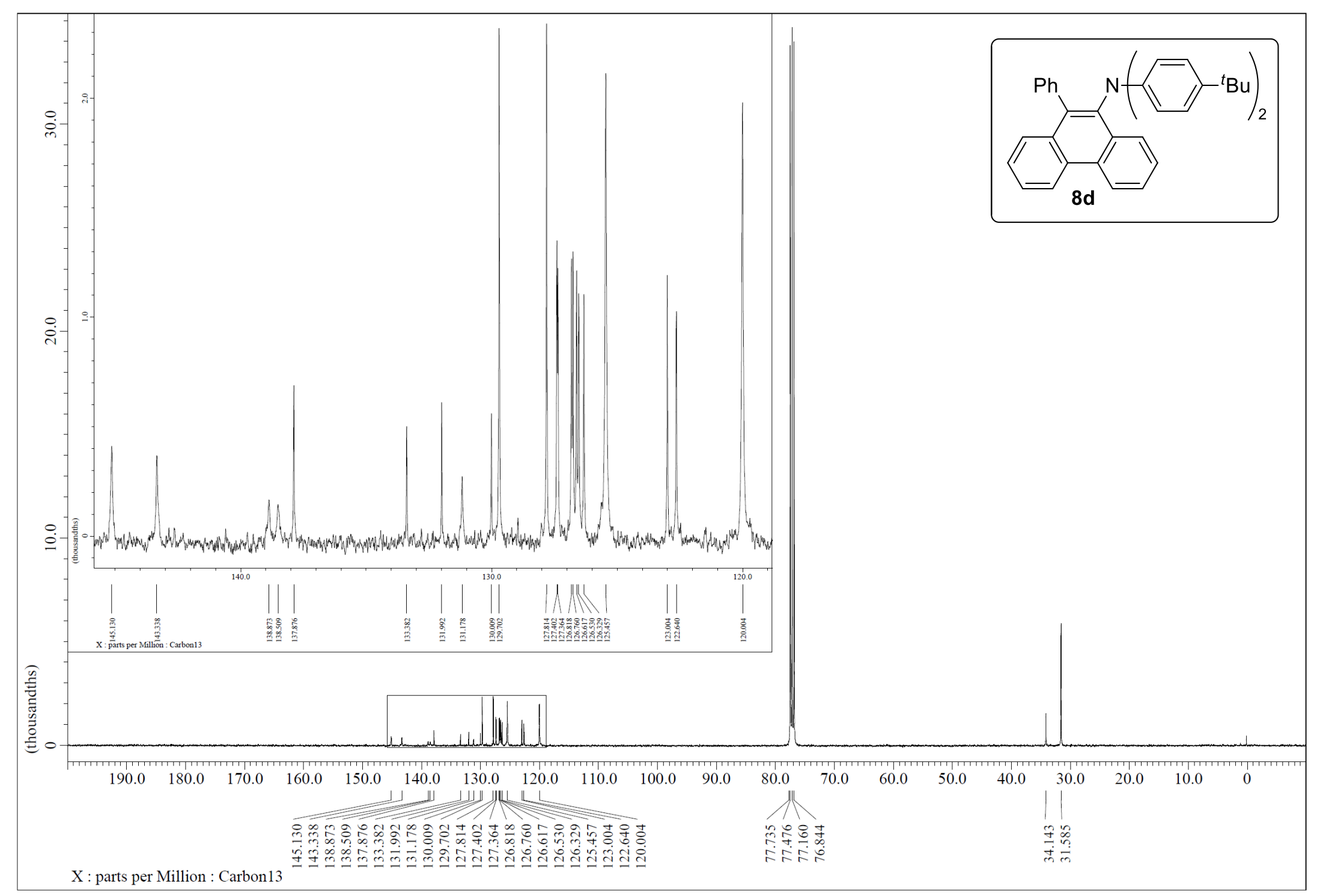

Figure S104. ${ }^{13} \mathrm{C}\left\{{ }^{1} \mathrm{H}\right\}$ NMR $(101 \mathrm{MHz})$ spectrum of $\mathbf{8 d}\left(\mathrm{CDCl}_{3}, \mathrm{rt}\right)$ 


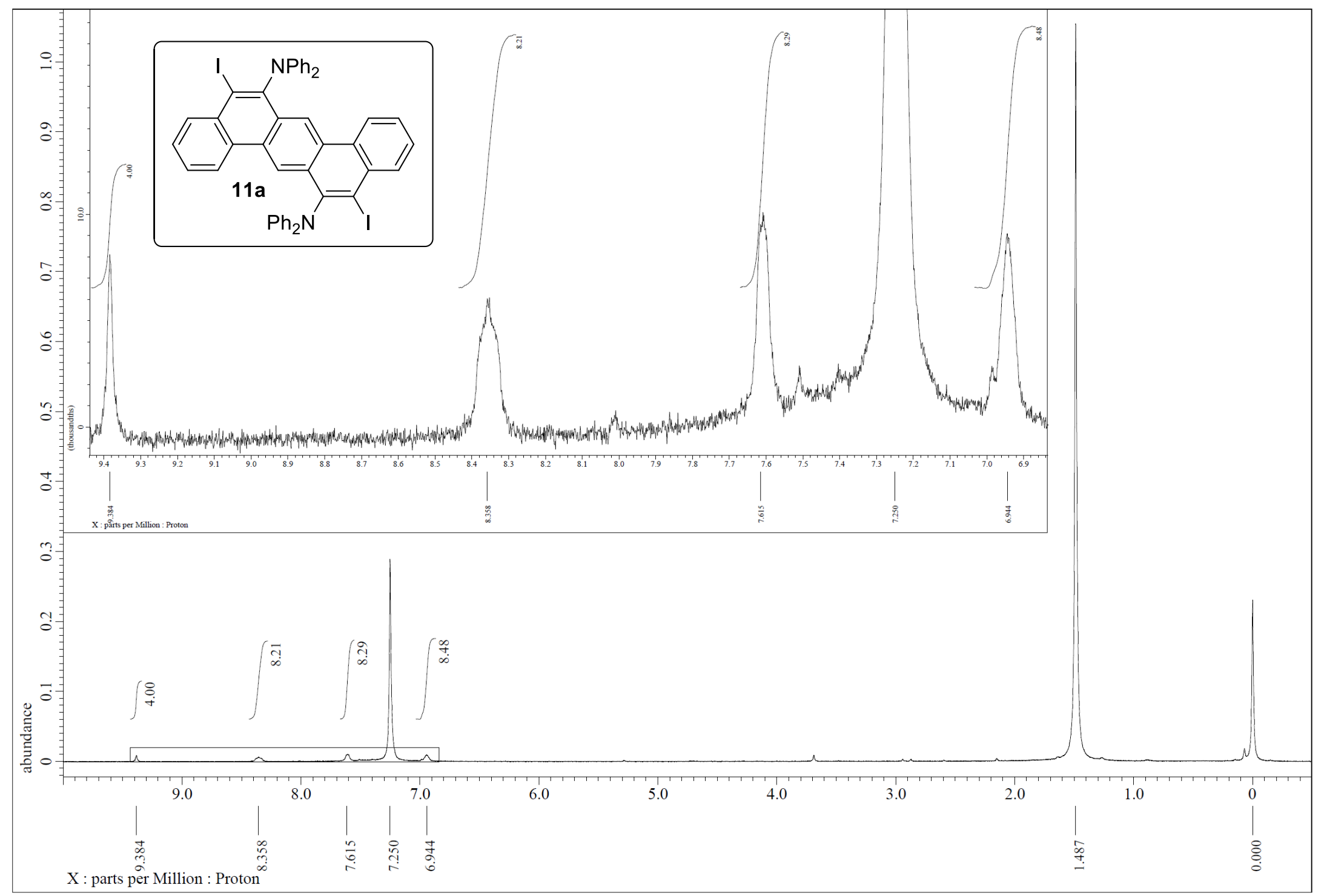

Figure S105. ${ }^{1} \mathrm{H}$ NMR (400 MHz) spectrum of $11 a\left(\mathrm{CDCl}_{3}, 60{ }^{\circ} \mathrm{C}\right)$ 


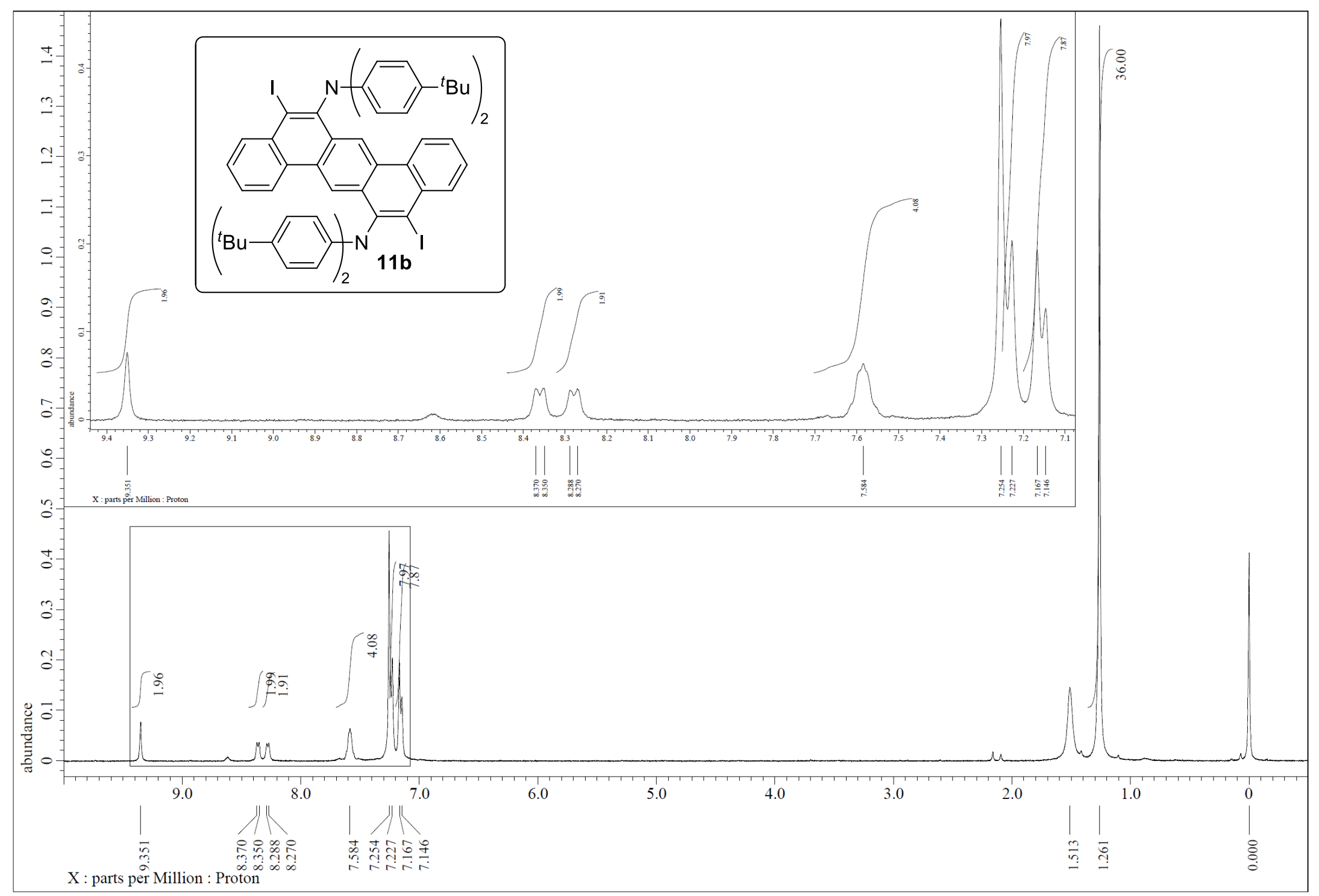

Figure S106. ${ }^{1} \mathrm{H}$ NMR (400 MHz) spectrum of $\mathbf{1 1 b}\left(\mathrm{CDCl}_{3}, 60{ }^{\circ} \mathrm{C}\right)$ 


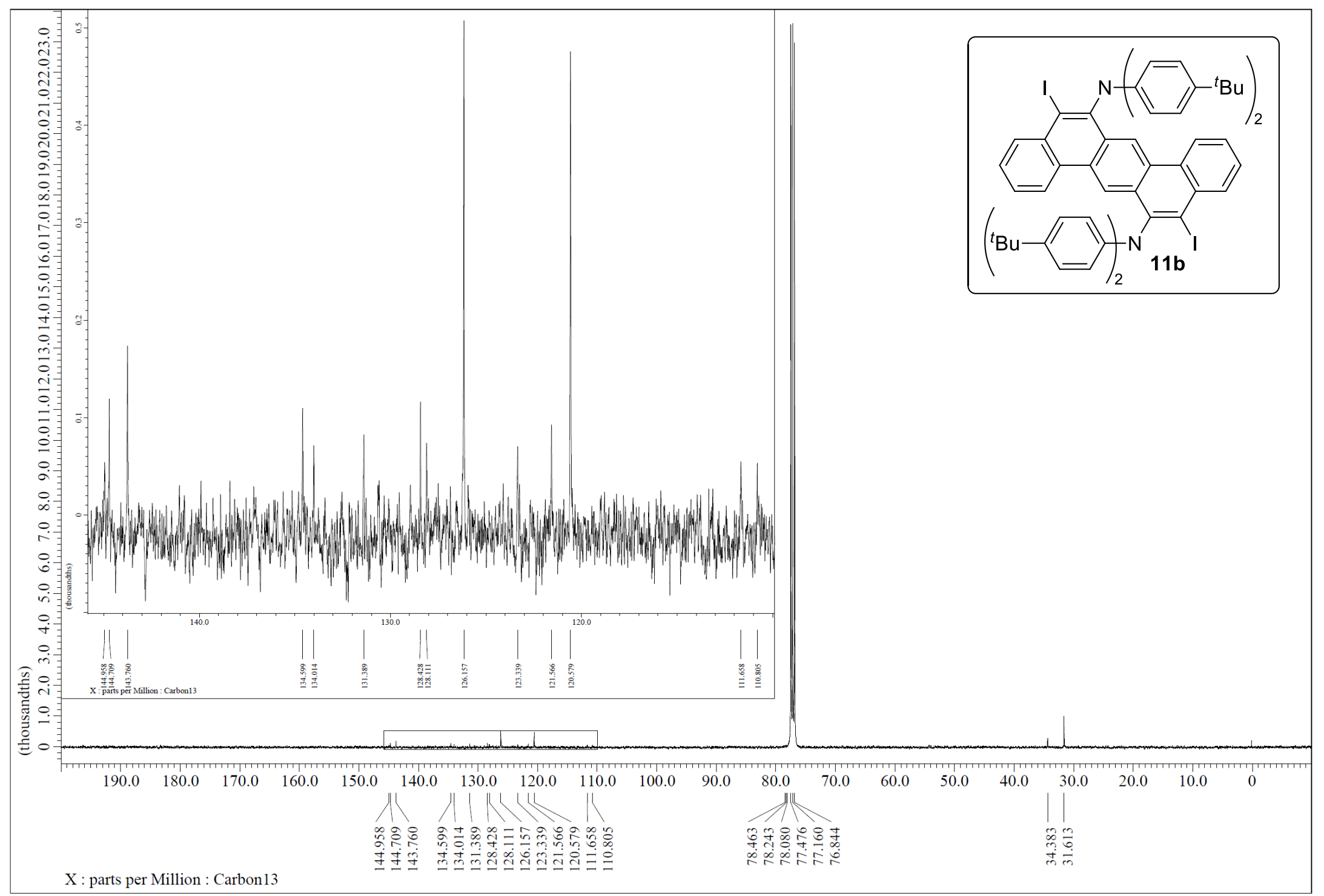

Figure S107. ${ }^{13} \mathrm{C}\left\{{ }^{1} \mathrm{H}\right\}$ NMR (101 MHz) spectrum of $\mathbf{1 1 b}\left(\mathrm{CDCl}_{3}, 60{ }^{\circ} \mathrm{C}\right)$ 


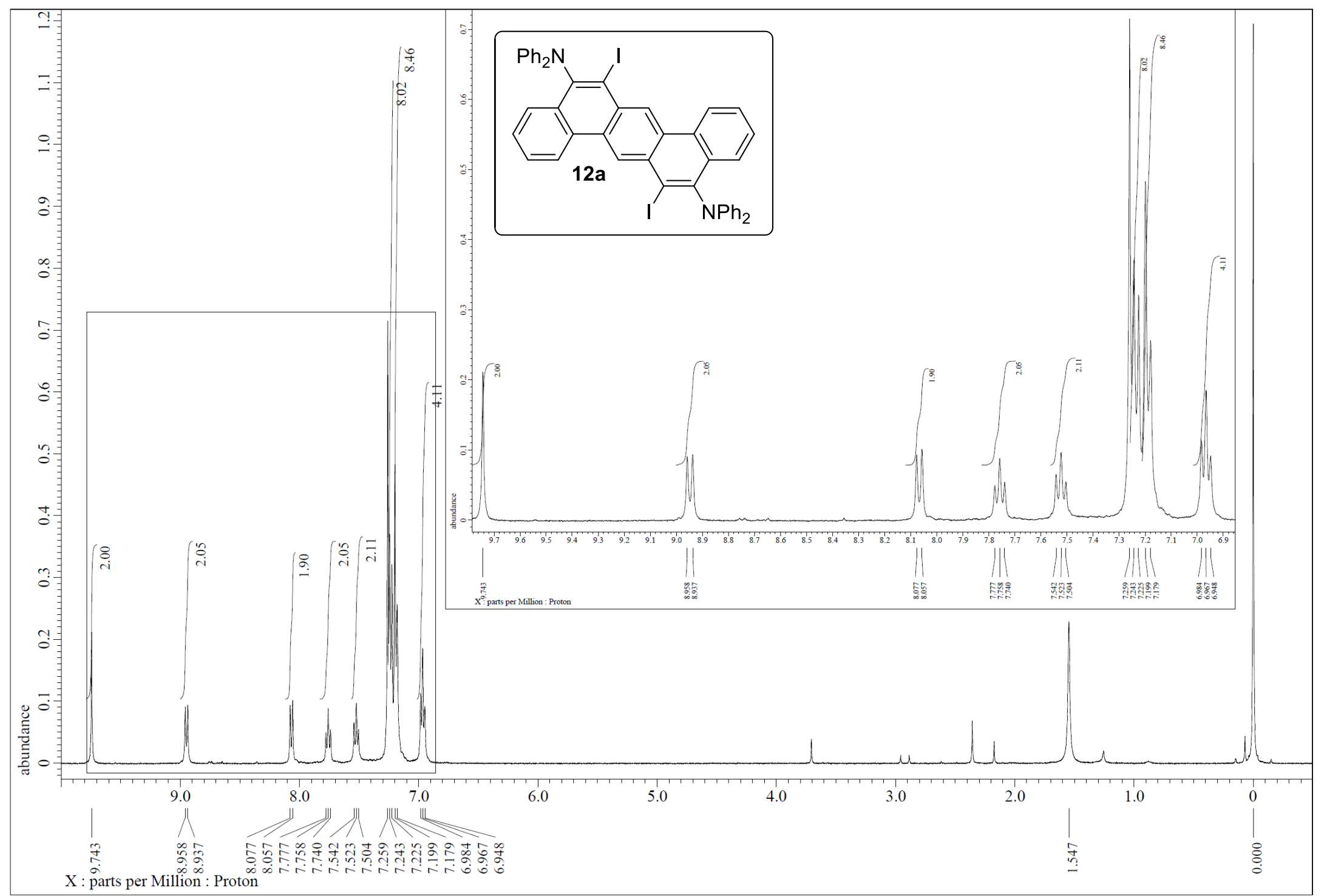

Figure S108. ${ }^{1} \mathrm{H}$ NMR $(400 \mathrm{MHz})$ spectrum of $\mathbf{1 2 a}\left(\mathrm{CDCl}_{3}\right.$, rt) 


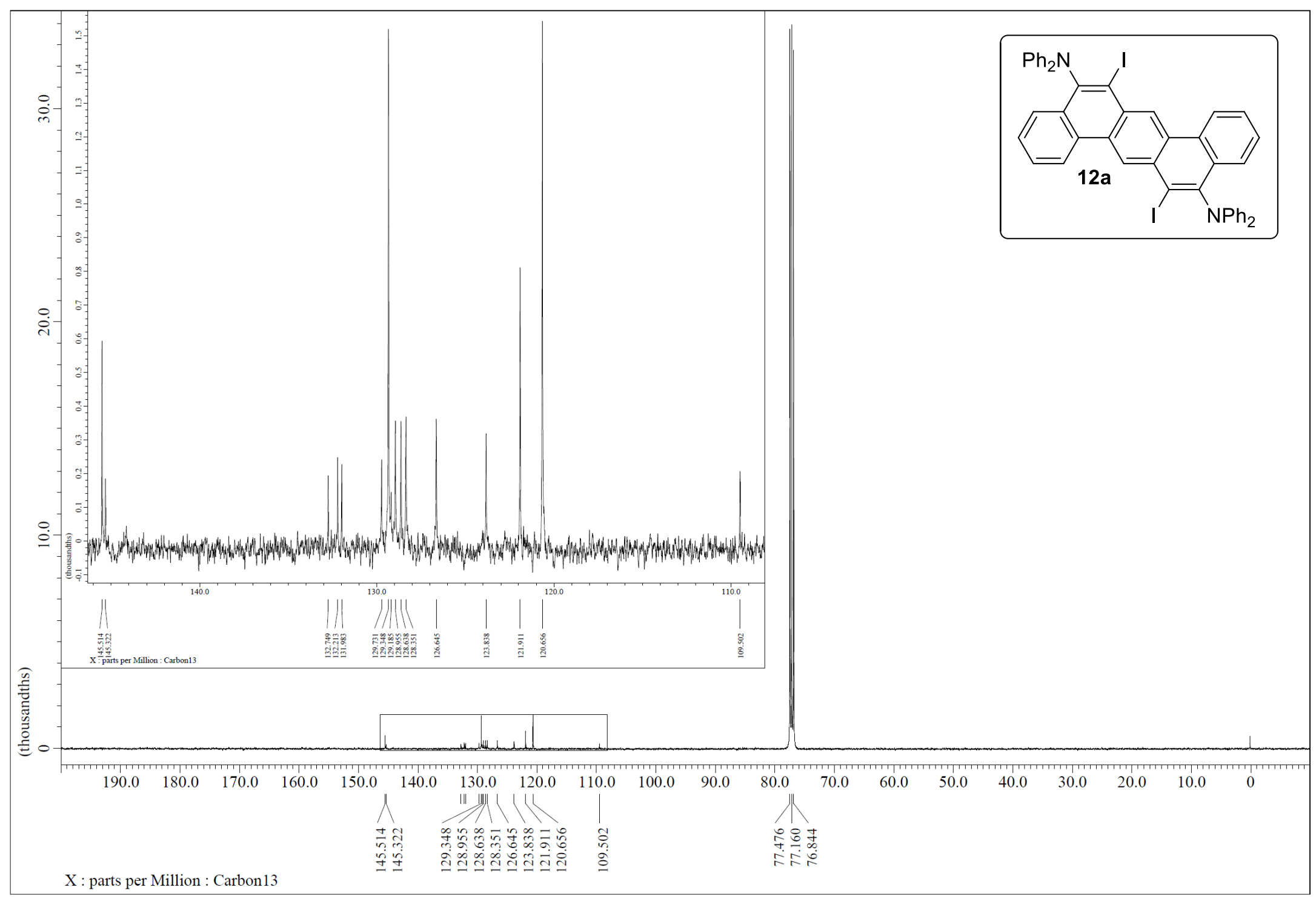

Figure S109. ${ }^{13} \mathrm{C}\left\{{ }^{1} \mathrm{H}\right\}$ NMR $(101 \mathrm{MHz})$ spectrum of 12a $\left(\mathrm{CDCl}_{3}, \mathrm{rt}\right)$ 


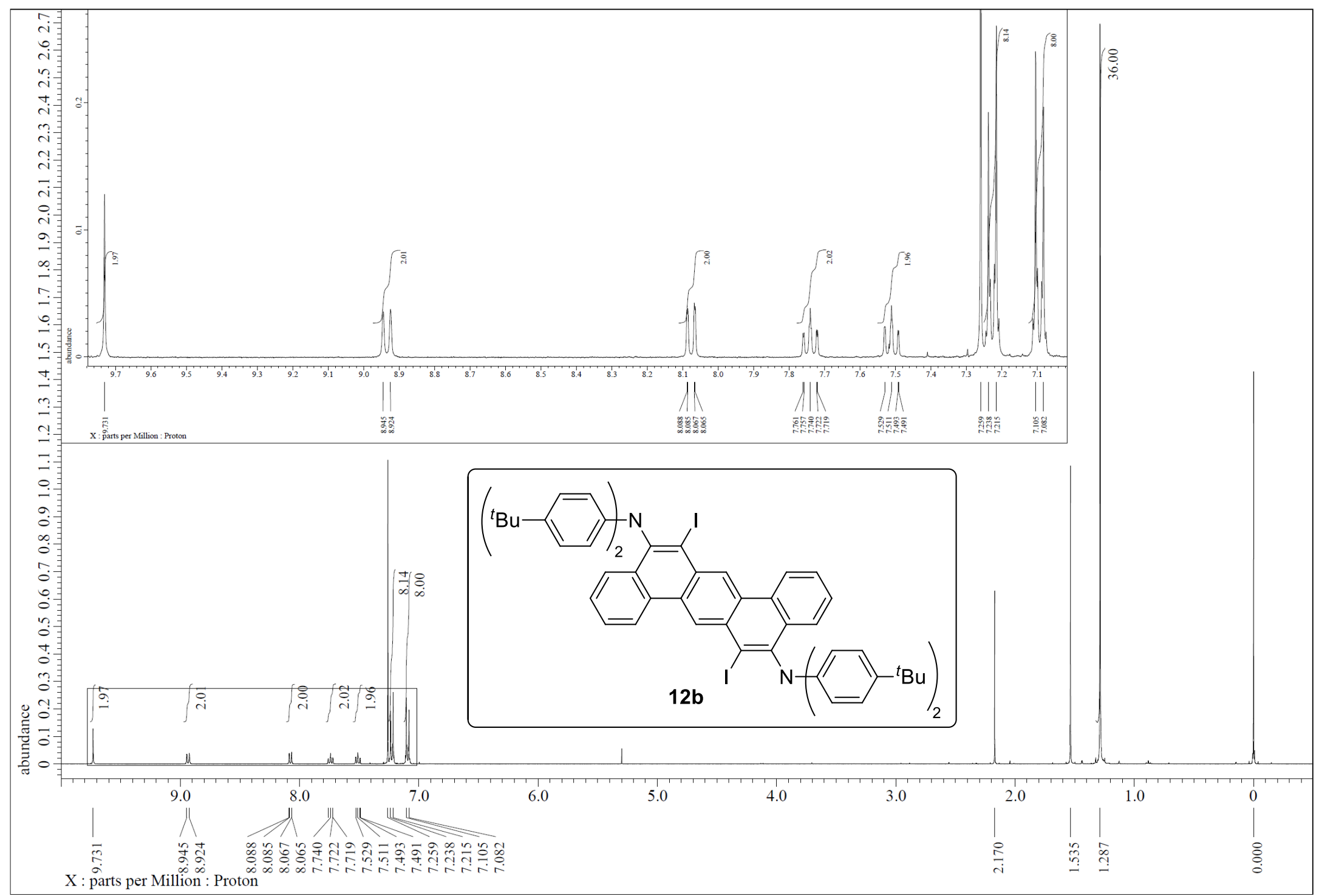

Figure S110. ${ }^{1} \mathrm{H}$ NMR $(400 \mathrm{MHz})$ spectrum of $\mathbf{1 2 b}\left(\mathrm{CDCl}_{3}, \mathrm{rt}\right)$ 


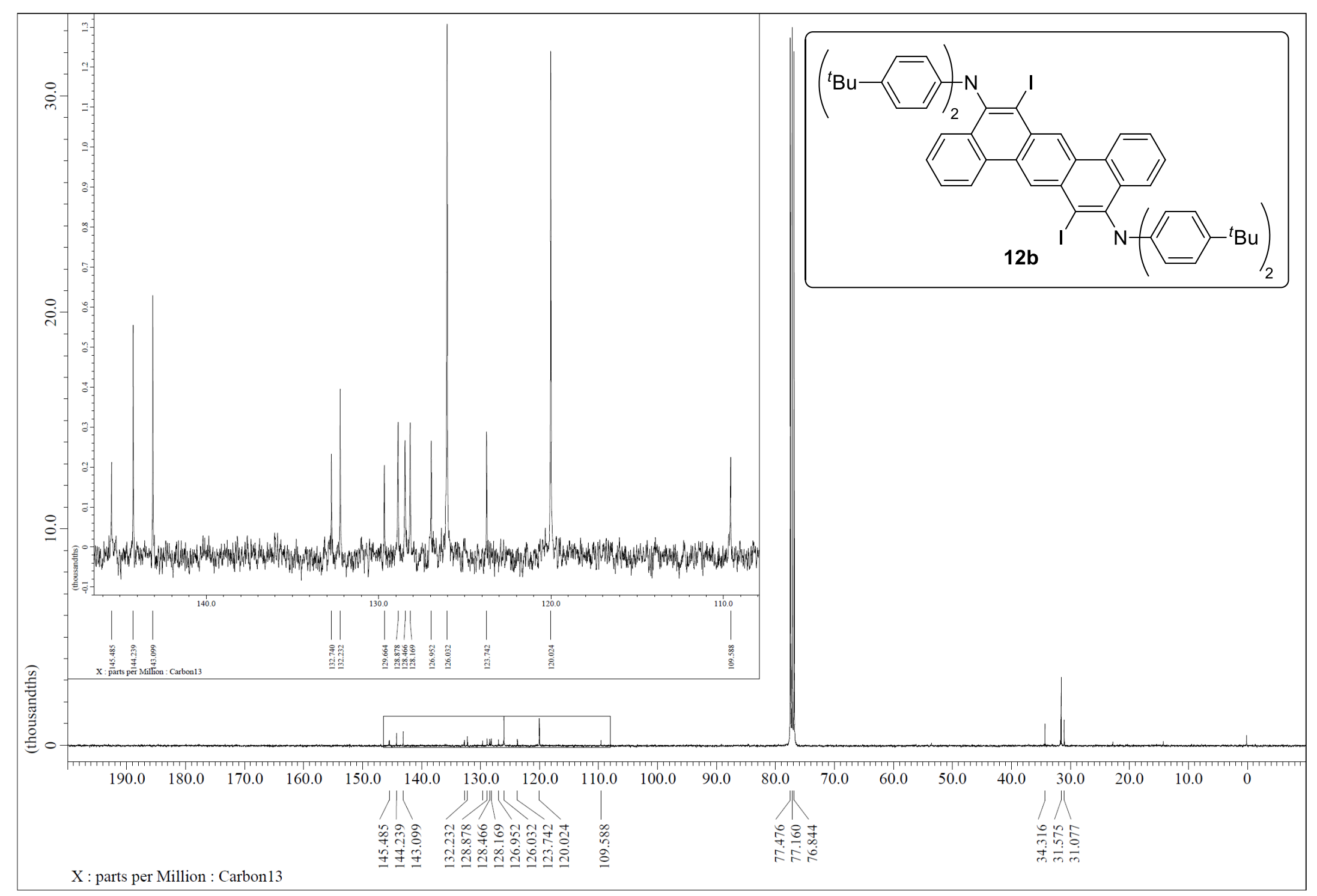

Figure S111. ${ }^{13} \mathrm{C}\left\{{ }^{1} \mathrm{H}\right\}$ NMR $(101 \mathrm{MHz})$ spectrum of $\mathbf{1 2 b}\left(\mathrm{CDCl}_{3}, \mathrm{rt}\right)$ 


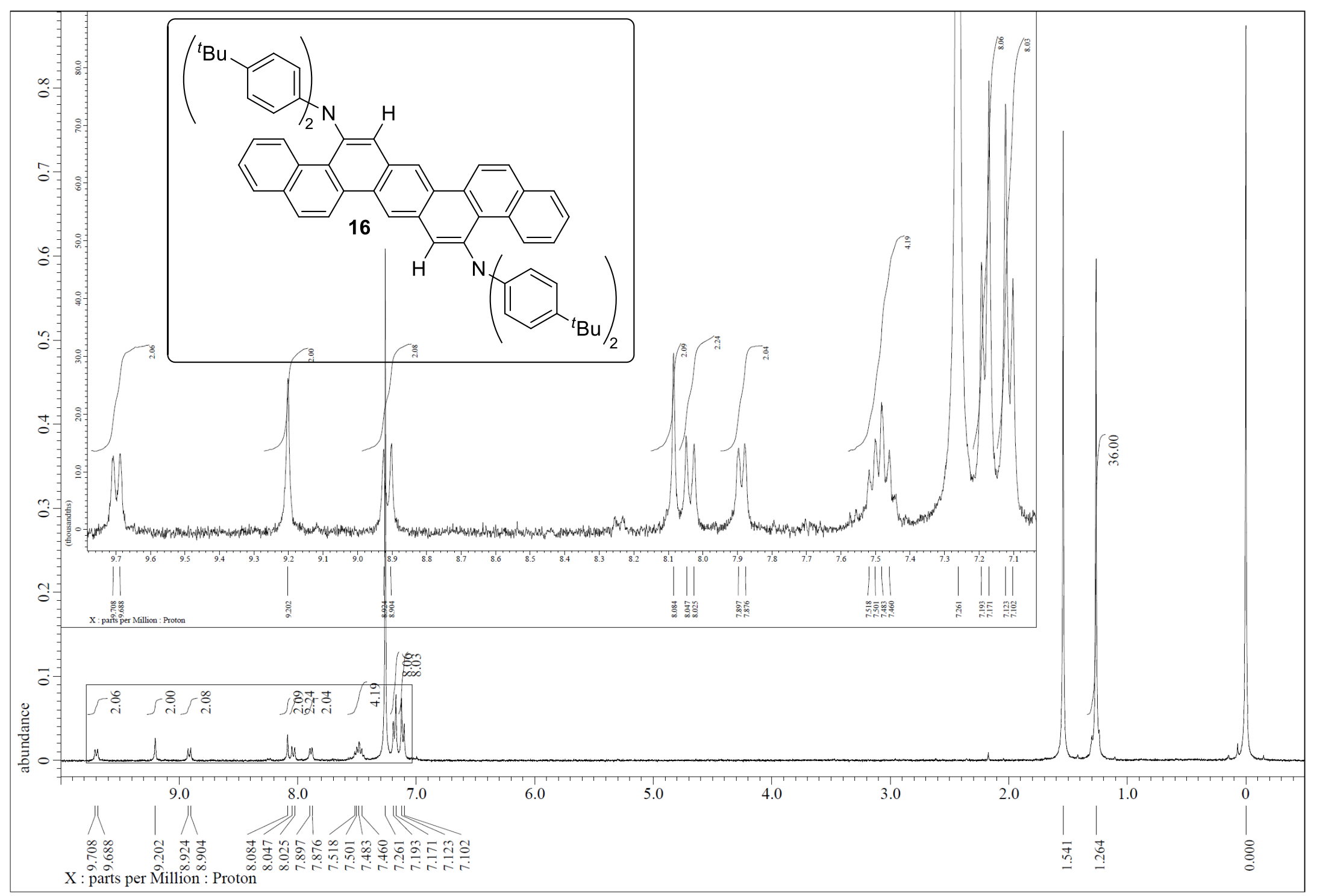

Figure S112. ${ }^{1} \mathrm{H}$ NMR $(400 \mathrm{MHz})$ spectrum of $\mathbf{1 6}\left(\mathrm{CDCl}_{3}, \mathrm{rt}\right)$ 


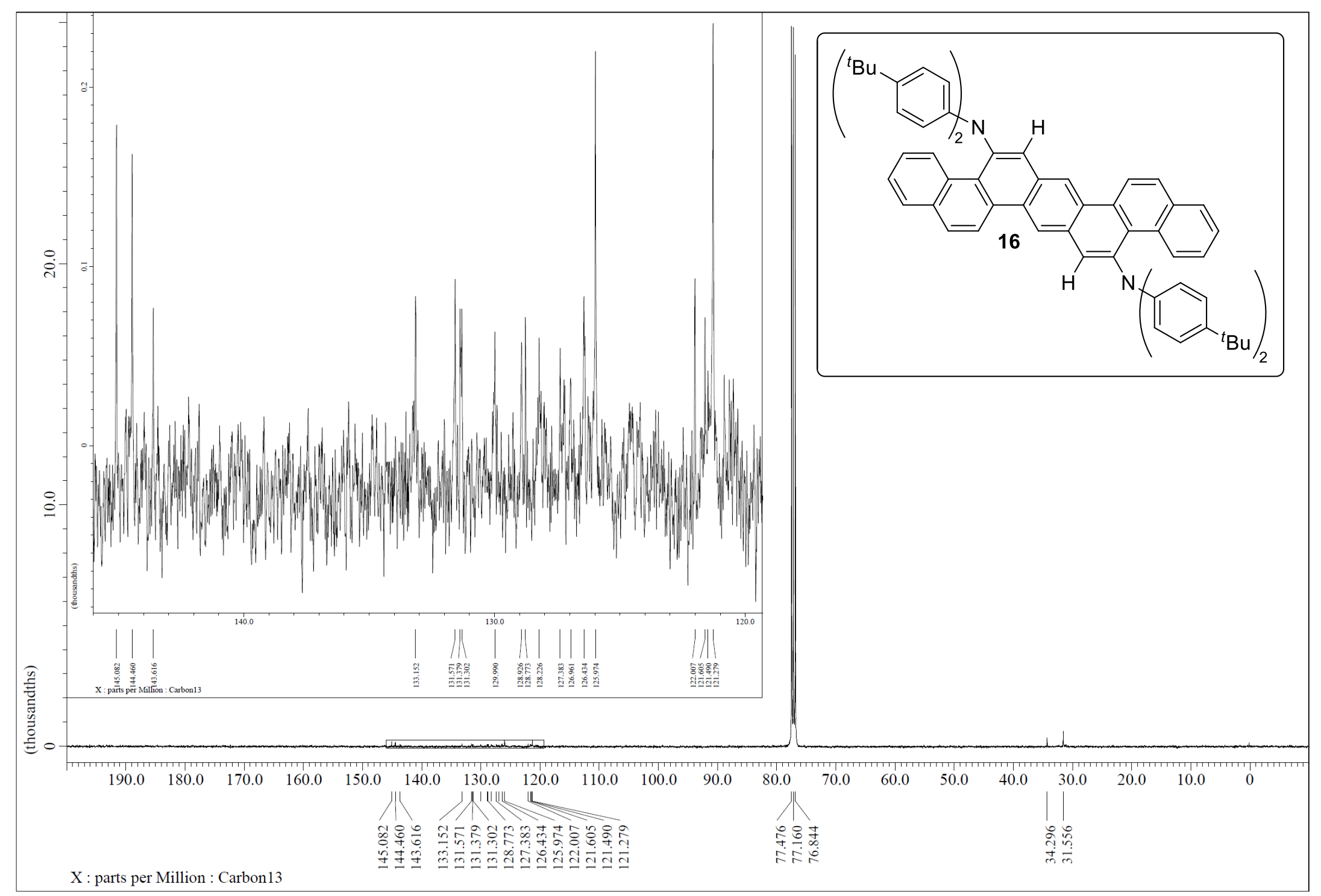

Figure S113. ${ }^{13} \mathrm{C}\left\{{ }^{1} \mathrm{H}\right\}$ NMR $(101 \mathrm{MHz})$ spectrum of $\mathbf{1 6}\left(\mathrm{CDCl}_{3}, \mathrm{rt}\right)$ 


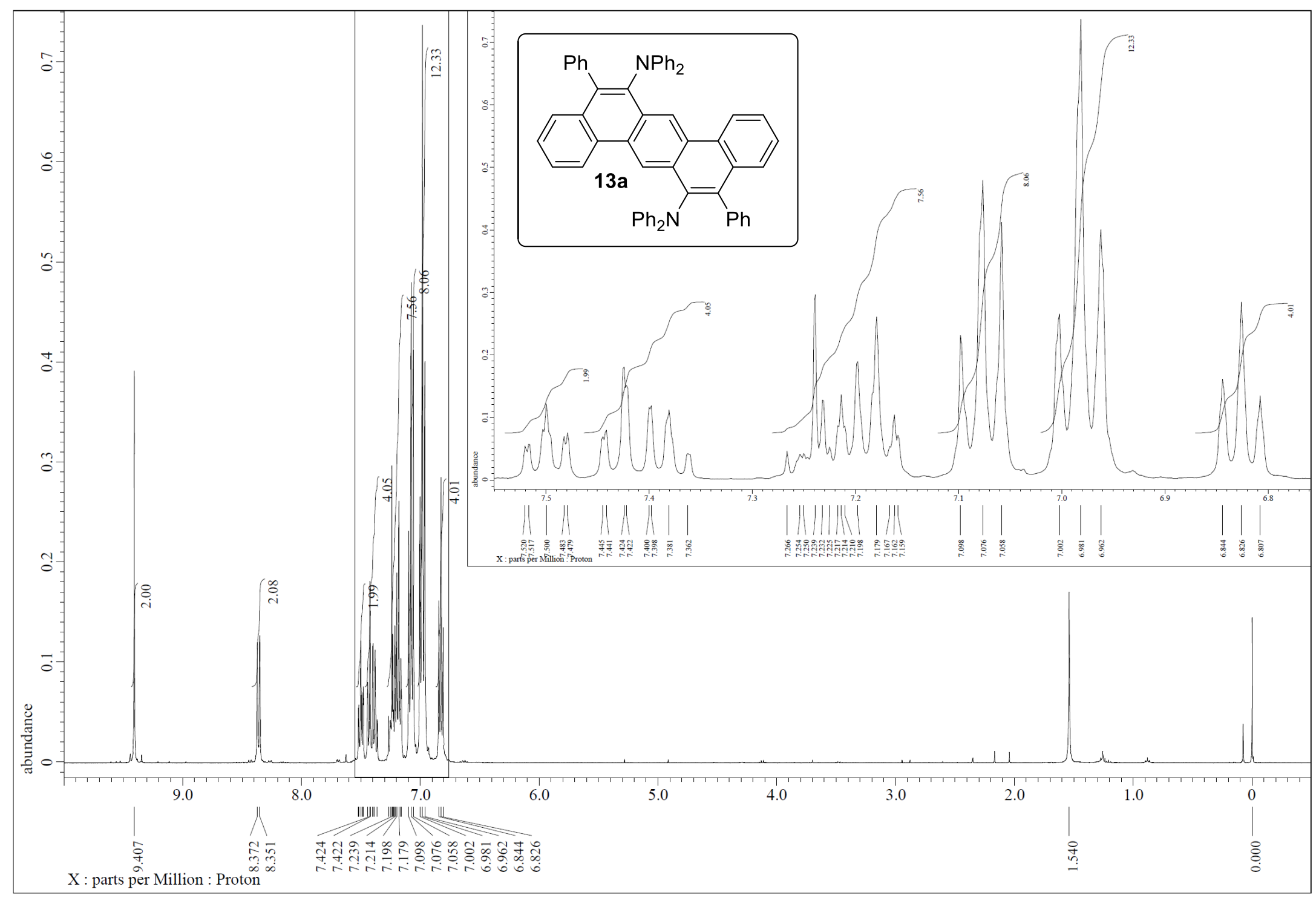

Figure S114. ${ }^{1} \mathrm{H}$ NMR $(400 \mathrm{MHz})$ spectrum of 13a $\left(\mathrm{CDCl}_{3}, \mathrm{rt}\right)$ 


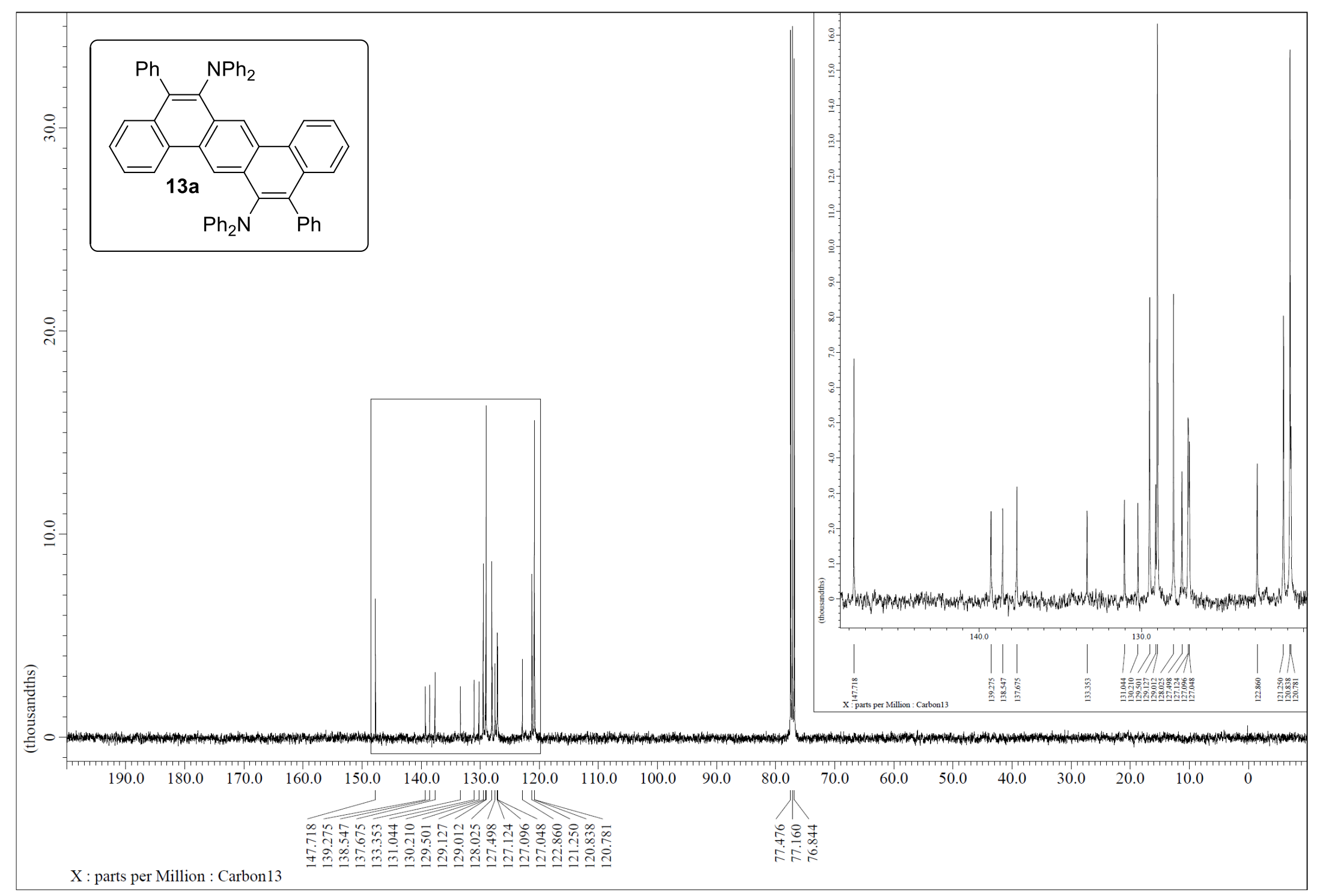

Figure S115. ${ }^{13} \mathrm{C}\left\{{ }^{1} \mathrm{H}\right\}$ NMR $(101 \mathrm{MHz})$ spectrum of $\mathbf{1 3 a}\left(\mathrm{CDCl}_{3}, \mathrm{rt}\right)$ 


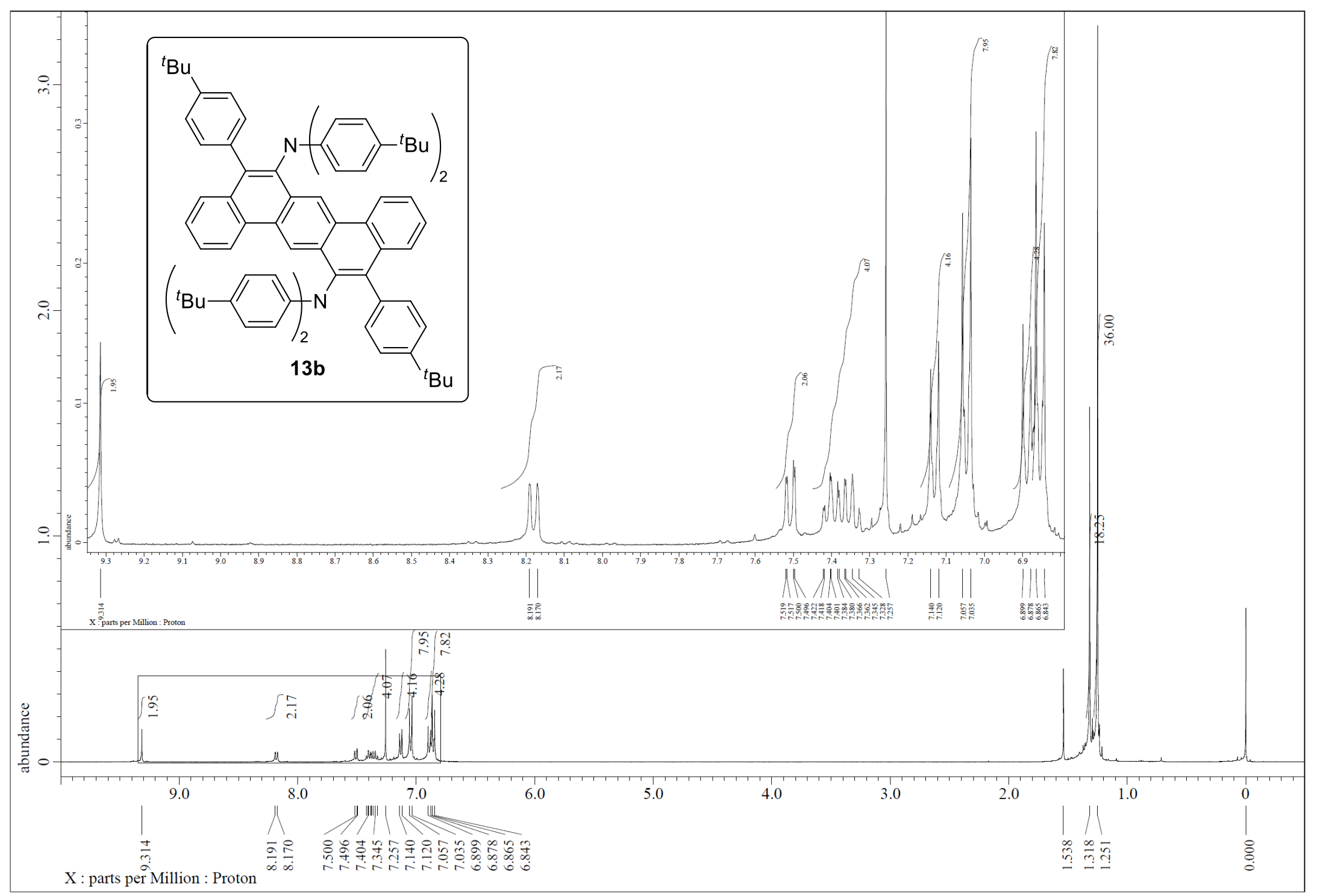

Figure S116. ${ }^{1} \mathrm{H}$ NMR $(400 \mathrm{MHz})$ spectrum of $\mathbf{1 3 b}\left(\mathrm{CDCl}_{3}, \mathrm{rt}\right)$ 


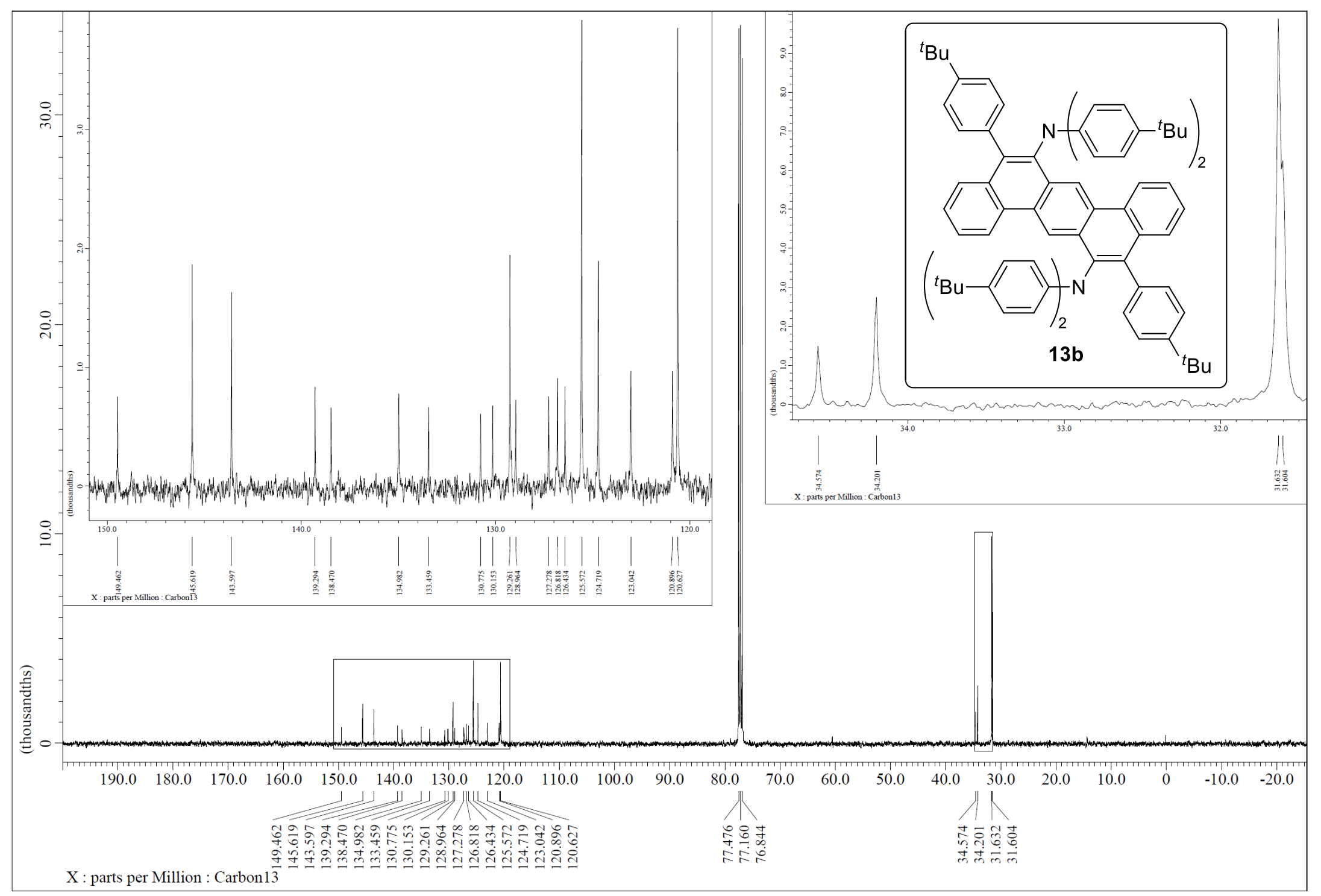

Figure S117. ${ }^{13} \mathrm{C}\left\{{ }^{1} \mathrm{H}\right\}$ NMR $(101 \mathrm{MHz})$ spectrum of $\mathbf{1 3 b}\left(\mathrm{CDCl}_{3}, \mathrm{rt}\right)$ 


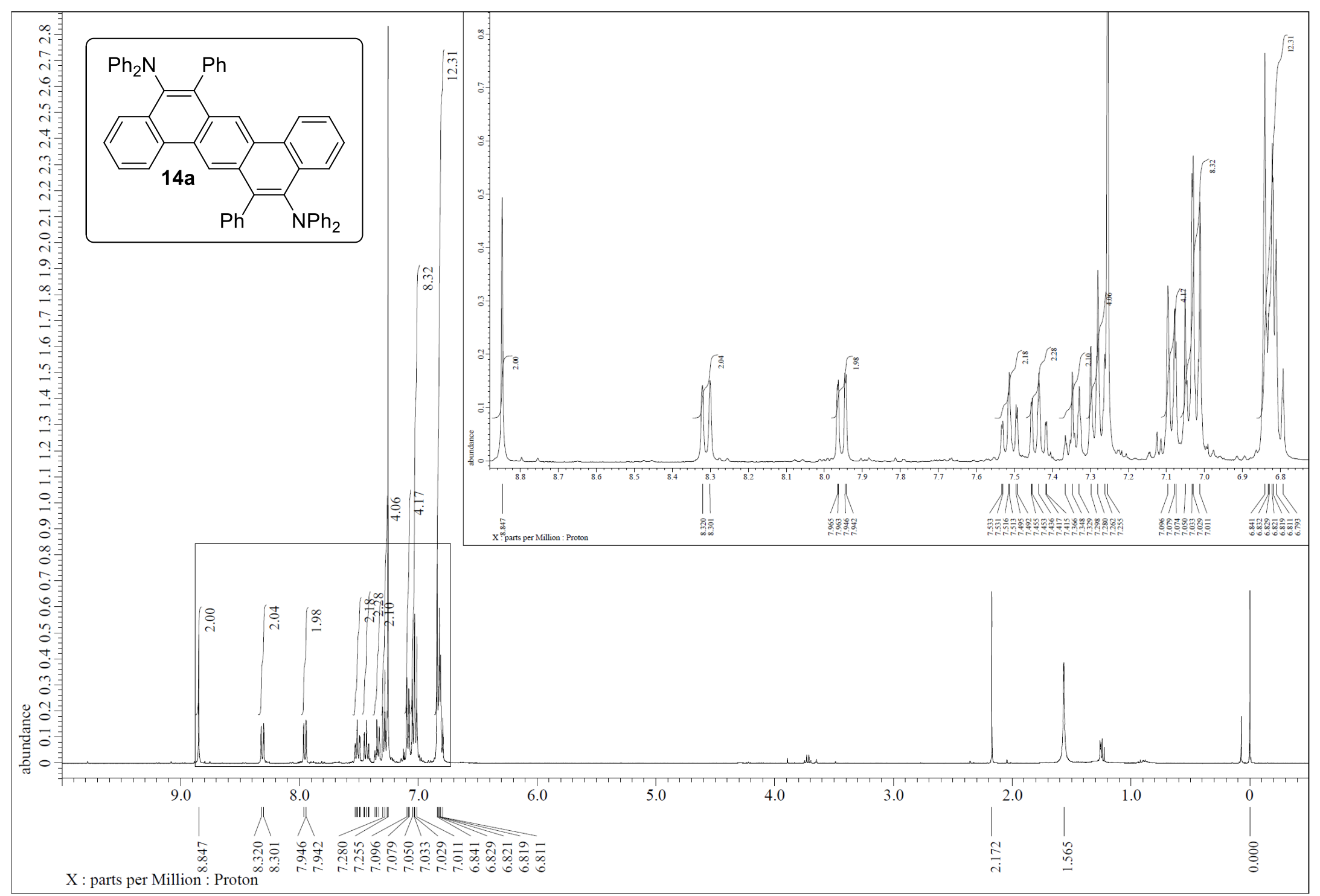

Figure S118. ${ }^{1} \mathrm{H}$ NMR $(400 \mathrm{MHz})$ spectrum of $\mathbf{1 4 a}\left(\mathrm{CDCl}_{3}, \mathrm{rt}\right)$ 


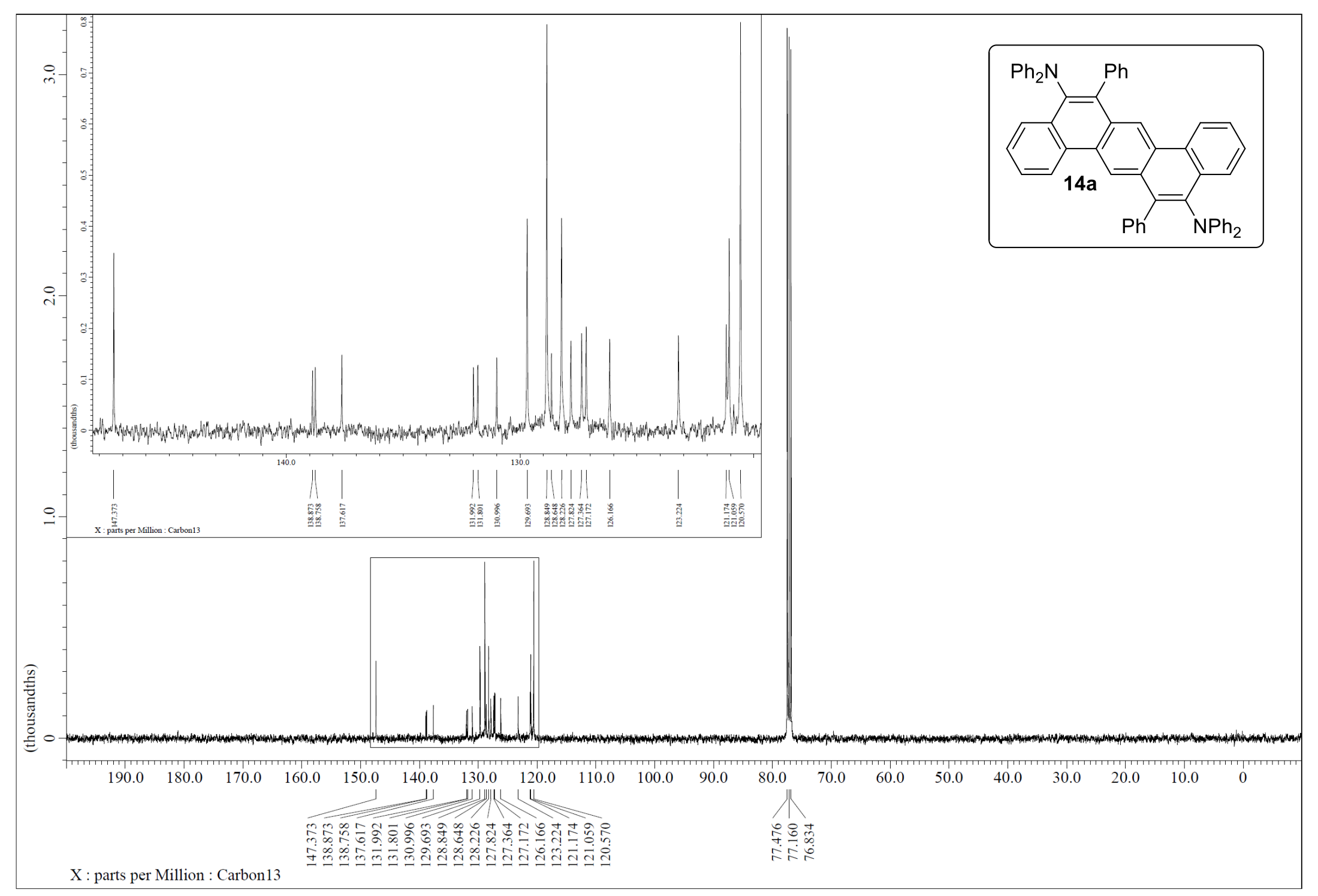

Figure S119. ${ }^{13} \mathrm{C}\left\{{ }^{1} \mathrm{H}\right\}$ NMR $(101 \mathrm{MHz})$ spectrum of $\mathbf{1 4 a}\left(\mathrm{CDCl}_{3}, \mathrm{rt}\right)$ 


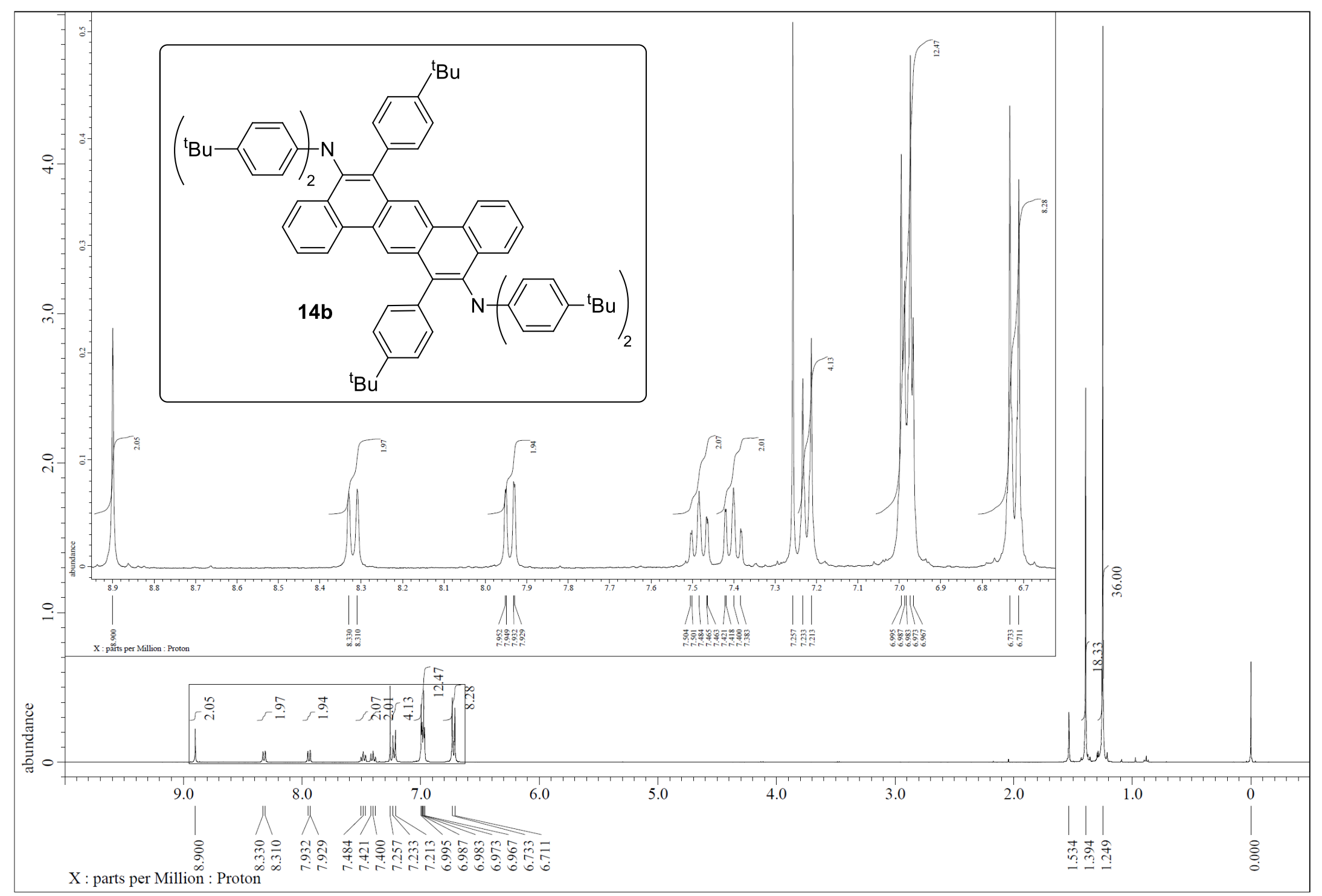

Figure S120. ${ }^{1} \mathrm{H}$ NMR $(400 \mathrm{MHz})$ spectrum of $\mathbf{1 4 b}\left(\mathrm{CDCl}_{3}, \mathrm{rt}\right)$ 


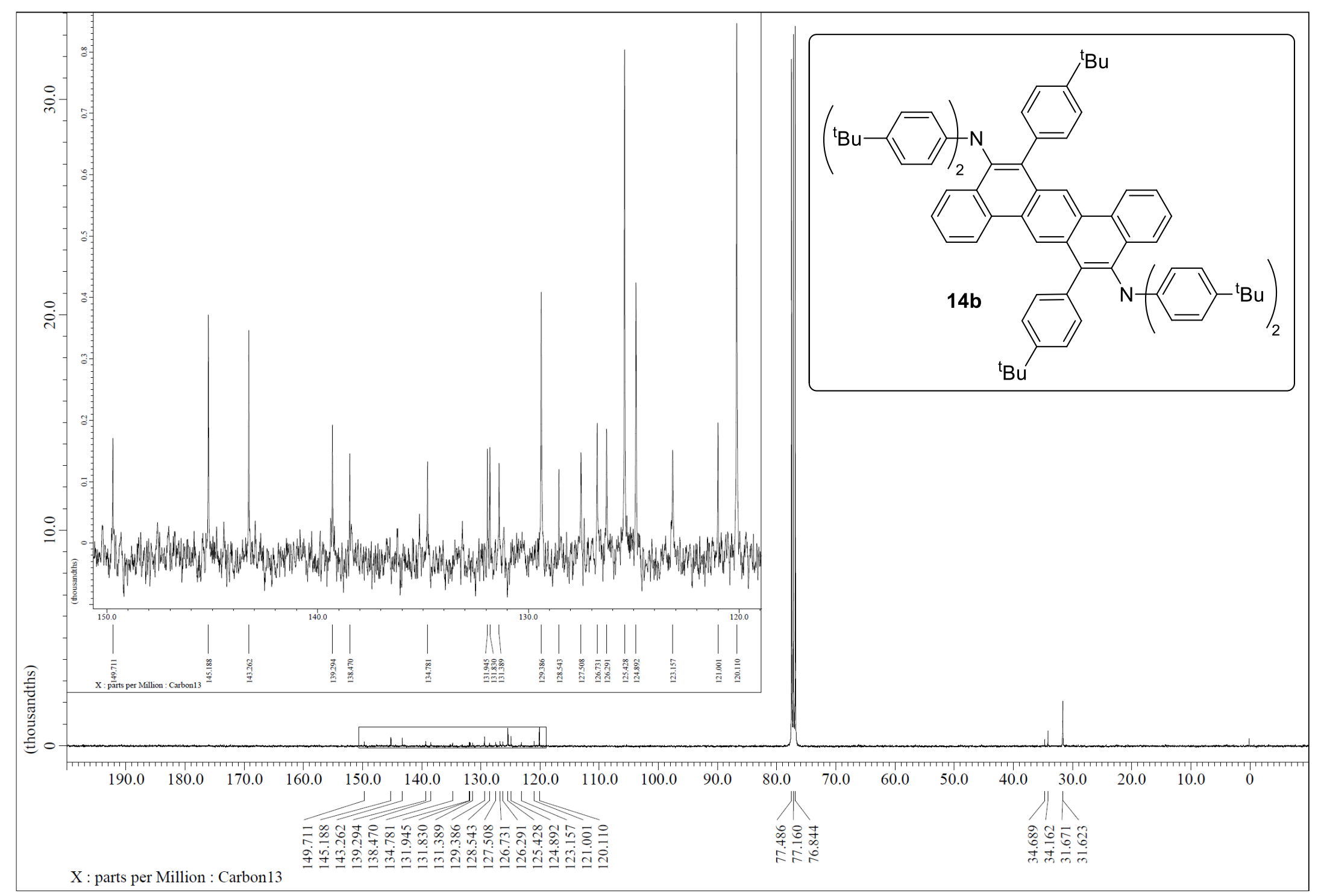

Figure S121. ${ }^{13} \mathrm{C}\left\{{ }^{1} \mathrm{H}\right\} \mathrm{NMR}(101 \mathrm{MHz})$ spectrum of $\mathbf{1 4 b}\left(\mathrm{CDCl}_{3}, \mathrm{rt}\right)$ 